\title{
Ring Expansions of Cyclopropanated Carbohydrates
}

\author{
By \\ Rhys Antony Batchelor
Victoria
UNIVERSITY OF WELLINGTON
Te Whare Wānanga
圈圈: \\ o te Ūpoko o te Ika a Māui
}

\begin{abstract}
A thesis
Submitted to Victoria University of Wellington

in fulfilment of the requirements for the degree of

Doctor of Philosophy

In Chemistry
\end{abstract}

Victoria University of Wellington

2008 


\begin{abstract}
The combination of cyclopropanes and carbohydrates functionality within a single molecule gives the synthetic chemist an interesting combination of reactivity and chirality to expand further upon. However, until recently relatively little work has been done in this field. Following the report of methodology to convert cyclopropanated carbohydrates into oxepines in high yields, this investigation set out to improve the selectivity of the rearrangement and also investigate the further utility of the subsequent products.
\end{abstract}

Focused investigation of the starting substrates and reaction conditions led to a minor adaptation of the previously successful methodology for the ring expansion of cyclopropanated glycals. This resulted in a substantial increase in the selectivity of the reaction to generate oxepine rings in good to excellent yields with a range of nucleophiles. One of the oxepines was subsequently chosen for elaboration into a range of synthons for further investigations. These yielded a variety of oxepanes in good yields with well defined stereo- and regioselective outcomes.

In the course of these reactions several unexpected products were isolated. These were further investigated with labelling experiments and a mechanism for their formation was proposed.

Finally the methodology was applied to the total synthesis of a naturally occurring oxepine containing compound. Despite the apparent simplicity of the target, the effort was ultimately unsuccessful. 


\section{Acknowledgements}

It's not easy doing a $\mathrm{PhD}$ thesis when you are only part time, but I've had a stack of support from my peers and friends. So many to thank and so little space. If it was the Oscars the music would start playing somewhere at the bottom of this page.

Every candidate needs a good $\mathrm{PhD}$ supervisor. I've been lucky to have 3 .

Firstly Dr John Hoberg for initially taking me on as a part time student and actually getting me to think like a chemist. His example of inspiring students to work long hours (by actually putting the hours in himself) and his amazing skill at remembering obscure mechanisms (or at least convincing the rest of us that he knew what he was talking about) have left a lasting impression on me.

After John returned to his homeland I was lucky enough to have Associate Professor Peter Northcote agree to become my supervisor. Despite his claims that he was not a synthetic chemist he made some excellent suggestions during the course of this thesis. I've learn't an awful lot about NMR spectroscopy from him. We've also been through a lot working together in the undergraduate organic labs over the last 13 years. I'm glad to have had him as a mentor and friend.

Some $\mathrm{PhD}$ students never get to see their second supervisor; I never got that luxury. Dr Paul Teesdale-Spittal was always there with a helpful suggestion or selection of puns. Despite his promotion to head of SBS he always seemed to have time to escape to talk chemistry with our research group. His hard work doing the final proof readings of this work while on holiday were greatly appreciated. 
I'd also like to thank Dr Joanne Harvey for her generous input. Her help teaching me how to write in scientific language (a skill I'd much neglected) was greatly appreciated, as was her time even when busy to assist me. Dr Mattie Timmer provided some extremely useful input during the final stages of writing up. Dr Laine Cousins for her quick proof reading efforts and long friendship (and more than a few beers over the years). Dr Brendan Burkett doesn't escape. His enthusiasm for teaching in the labs, and being able to take it when Australia lose at sport have been great. I'd also like to thank Dr Andy Falshaw, emeritus Professor Brian Halton and Professor Robin Ferrier, for their assistance and insights over the years.

I've also been lucky that the heads of school have tolerated me during my extended time doing my $\mathrm{PhD}$, so thanks to Associate Professor Jim Johnston and Professor John Spencer for supporting me by letting me spend my time where I wanted as long as the lab courses ran without problems.

The students I've worked with in the research group over those long years don't escape a mention; Rona, Bridget, Andreas, Silvia, Shivali, Anna, Ben, Katherine, Wayne, Corin, Ying, James, and Darrin. Also Lynton, big Emma, little Emma, and Russel who came afterwards. We had some good times (and some not so good times) over the long days in the lab, and I wouldn't swap most of it for anything (well, apart from Lyntons music). Thanks for everything.

I've been very privileged to work with a great group of people for 14 years, which in this day and age is something of a rarity. Gordon, Dave, Teresa, and Jackie; you guys have 
helped me so much over the last 7 years to achieve this, I could not have done it without you, and I will really miss working along side you all.

Then there are the rest of the Chemists; Thomas, Andy, Mike, John, Wendy, Joanna, Jonno, Mathew, Kate (honorary), Alan, Manu, Grant, and some of the guys from times past; Fake, Paul, Horst, Rob, Steve M, as well as Tom and Les. You've all helped me in some way over the years, whether in work or just going out for a beer on Friday night, and its always been much appreciated even if I've never said as much. To everyone else in SCPS that I haven't mentioned thank you anyway just for being a great bunch of people to work with.

I was extremely lucky to have a place of escape where it didn't matter what I did for the rest of the week, but what I was like as a person. It was nice to have that little bastion of (in) sanity so I'd like to thank the guys; Peter C, Peter P, Rob, Paul, Greg, Chris, Richard, Kelly, Luke and Keith (and anyone else I met wargaming) for being the best group of mates a man could want and providing plenty of hilarity when I really needed it.

Also some old friends; Darryl, Carol, Susan, Craig, Vicky and Douglas. It doesn't seem like 30 years at all. Thanks for still being there from time to time. I'd also like to thank my parents Mike and Annette for supporting me over the years no matter what I decided to do, and Evan and Alison, Vic, Jocelyn, Rose, Gary and Kyle.

Before I finally shuffle off the stage there's one person left to thank. To the most important person in my life, who has put up with late nights, bad moods, depression, elation, and general untidiness over the years (far longer than she expected it would last). None of this would have been possible without my wife Janeen. This is for you, babe. 


\section{Table of Contents}

$\begin{array}{lll}\text { Title page } & \text { I }\end{array}$

\begin{tabular}{ll|l} 
Abstract & II
\end{tabular}

Acknowledgements III

$\begin{array}{ll}\text { Table of Contents } & \text { VI }\end{array}$

$\begin{array}{ll}\text { List of Reaction Schemes } & \text { X }\end{array}$

$\begin{array}{ll}\text { List of Figures } & \text { XV }\end{array}$

$\begin{array}{lc}\text { List of Tables } & \text { XIX }\end{array}$

$\begin{array}{ll}\text { List of Abbreviations } & \text { XX }\end{array}$

Chapter 1: Introduction 1

1.1 An introduction to carbohydrates 1

1.2 Conventions for the description of carbohydrate 4 structure and conformers

1.3 Glycosidic linkages 6

$\begin{array}{lll}1.4 & \text { Cyclopropanation of carbohydrates } & 17\end{array}$

$1.5 \quad$ Reactions of cyclopropanated carbohydrates 23

1.6 Oxepine rings in naturally occurring compounds 27

1.7 Methods of formation of seven-membered rings 29

1.7.1 Seven-membered oxacycle formation via C-O 29 bond formation

1.7.2 Seven-membered oxacycle formation via C-C 30 bond formation

1.8 Ring expansion of cyclopropanated carbohydrates 35

$\begin{array}{lll}1.9 & \text { Aims and objectives } & 38\end{array}$

1.10 A note about NMR assignments in this thesis 39 
Chapter 2: Initial studies on the Ring Expansion of Cyclopropanated Glycals 40

2.1 Investigations of 6-O-TIPS protected cyclopropanated 41 galactal

2.2 Investigation of alternative leaving groups 43

2.2.1 Carbonate as the leaving group 43

2.2.2 Triflate as the leaving group 44

2.2.3 Mesylate as the leaving group $\quad 47$

2.2.4 One pot generation of the triflate leaving group $\quad 47$ and ring expansion

$\begin{array}{lll}2.3 & \text { In situ generation of the leaving group } & 48\end{array}$

2.3.1 In situ generation of leaving group under $\quad 48$ Mitsunobu conditions

2.4 Ring expansion of glucal 2.12 with generation of leaving 56 group in situ

2.5 Investigation of intramolecular nucleophile delivery 62

2.5.1 The tandem Tebbe- Claisen rearrangement 63

2.5.2 Ring expansion using modified Tebbe-Claisen $\quad 66$

$\begin{array}{lll}2.6 & \text { Summary } & 67\end{array}$

Chapter 3: Synthesis and ring expansions of cyclopropanated galactal and $\quad 68$ glucal derivatives

3.1 Synthesis and reactions of cyclopropanated di-tert-butyl 71 silyl galactal

3.1.1 Ring expansions of cyclopropane 3.4 72

3.1.2 Investigation of the role of sterics in the ring 76 expansion of cyclopropane 3.4

3.2 Ring expansions of cyclopropanated TBDPS protected 78 glucal 
3.2.1 Investigation of the role of sterics in the ring expansion of cyclopropane 3.16

3.3 Effect of the leaving group stereochemistry on the ring expansion

3.4 Comparison with glycal glycoside rearrangements

3.5 Summary

Chapter 4: Unexpected rearrangements obtained from ring expansions and mechanistic rational

4.1 Unexpected reaction products from ring expansions of cyclopropanated glucal 3.16

4.1.1 Reaction with carbon nucleophiles 3.9 and 3.10 87

4.1.2 Reaction of cyclopropane 3.16 with allyltrimethylsilane

4.2 Unexpected reaction products from ring expansions of cyclopropanated galactal 3.4

4.2.1 Reaction of TESH with cyclopropane 3.4

4.2.2 Investigation of the mechanism for the formation of oxepine 4.7

4.3 Summary

Chapter 5: Elaborations of oxepine 3.12

5.1 Halogen addition to oxepine 3.12

$\begin{array}{lll}\text { 5.1.1 } & \text { Bromination } & 104\end{array}$

$\begin{array}{lll}\text { 5.1.2 Bromohydrin formation } & 112\end{array}$

5.1.3 Mechanism of halogen addition to 3.12 114

$\begin{array}{lll}5.2 & \text { Epoxidation and derivatives } & 114\end{array}$

$\begin{array}{lll}\text { 5.2.1 } & \text { Epoxidation of oxepine 3.12 } & 114\end{array}$

5.2.2 Addition of sodium azide to epoxide 5.5 116 
5.4 Hydroboration of oxepine 3.12 120

5.5 Reduction of the ester of oxepine 3.12 122

$\begin{array}{lll}5.6 & \text { Summary } & 123\end{array}$

Chapter 6: Synthesis of a natural compound using the developed ring 125 expansion methodology

$\begin{array}{lll}\text { 6.1 Initial synthetic plan starting from cyclopropane } 3.3 & 127\end{array}$

6.2 Revised synthetic plan starting from cyclopropane 1.61 131

6.3 Modification of the initial synthetic route 132

6.4 Unexpected rearrangements during formation of diol $6.7 \quad 138$

6.4.1 Identification of unexpected products 138

6.4.2 Analysis of the mechanism of ring expansion of 141 cyclopropane 6.6

6.5 Summary

Chapter Seven: Conclusion

Chapter Eight: Experimental

Appendix A

Bibliography 


\section{List of Reaction Schemes}

1.1 Furanose and pyranose forms of D-glucose. 5

1.2 Hemiacetal formation leading to $\alpha$ and $\beta$ anomeric forms. 6

$\begin{array}{lll}1.3 & \text { Glycosylation by a glycosyl donor. } & 10\end{array}$

1.4 Glycosylation involving a glycal donor. 12

$\begin{array}{lll}\text { 1.5 } & \text { Ferrier glycal rearrangement. } & 12\end{array}$

1.6 Possible mechanisms involved in Ferrier rearrangements. 13

$\begin{array}{lll}1.7 & \text { Ferrier rearrangement of tri- } O \text {-acetyl-D-glucal with TMSallyl. } & 13\end{array}$

$\begin{array}{lll}1.8 & \text { Reaction of di-tert-butyl-glucal with TMSallyl and TMSOTf. } & 15\end{array}$

$\begin{array}{lll}\text { 1.9 } & \text { Fischer's total synthesis of glucose. } & 16\end{array}$

1.10 Leucomycin A3 1.14 showing stereocenters derived from D-glucose 16 starting material.

$\begin{array}{lll}\text { 1.11 Simmons-Smith cyclopropanations. } & 17\end{array}$

$\begin{array}{ll}\text { 1.12 Lorica's synthesis of cyclopropane 1.15. } & 19\end{array}$

$\begin{array}{ll}1.13 & \text { Hoberg's modified reaction scheme. }\end{array}$

$\begin{array}{lll}\text { 1.14 Synthesis of cyclopropane 1.20. } & 21\end{array}$

1.15 Possible paths for opening of the cyclopropane ring. 24

1.16 Installation of a gem-dimethyl moiety on the road to the epitholones. 25

1.17 Madsen's platinium catalysed opening of cyclopropanated glucal. 25

1.18 Formation of oxepines from expansion of cyclopropanes. 26

$\begin{array}{lll}\text { 1.19 Sugita's expansion of cyclopropapyranones.. } & 27\end{array}$

1.20 Suzuki's synthesis of isolaurepinnacin A and rogioloxepane A. 29

1.21 Oxepine formation involving Tebbe's metathasis. 30

1.22 Formation of oxepane rings leading to synthesis of hemibrevetoxin B. 31

1.23 Formation of oxepine ring with Grubbs catalyst. 32 
1.24 Jenkins and Ghost's oxepine formation.

1.25 Formation of oxepine ring with Grubbs catalyst. 33

1.26 Formation of oxepine ring by Prins cyclisation. 33

1.27 Nicolaou's photochemical generation of oxepine rings. 34

1.28 Hirama's photochemical generation of oxepine rings. 35

1.29 Formation of $[4,1,0]$ bicyclic system. 35

2.1 Hoberg's ring expansion methodology. 40

2.2 Ring expansion with interception of oxonium intermediate by internal 41 nucleophile.

2.3 Formation of cyclopropane 2.4.

2.4 Initial attempts toward a general method for ring expansion.

2.5 Formation of cyclopropanated galactal 2.5.

2.6 Attempted formation of triflate protected cyclopropane 2.6. 44

2.7 General structure of oxepine $\mathbf{2 . 7}$ formed from attempted triflate $\quad 45$ formation.

2.8 Bicyclic ring formation of 2.7a.

2.9 General proposed route to oxepines by in situ generation of triflate leaving group.

2.10 Mitsunobu reaction for conversion of an alcohol to a different functional group.

2.11 Proposed mechanism of the Mitsunobu reaction.

2.12 Possible products from Mitsunobu reaction.

2.13 Mitsunobu reaction in THF with $\mathrm{TMSN}_{3}$ as the nucleophile to generate 2.9.

2.14 Mitsunobu reaction in $\mathrm{MeCN}$ with $\mathrm{TMSN}_{3}$ as the nucleophile.

2.15 Reaction of 2.3 under Mitsunobu conditions with TMSallyl as nucleophile.

2.16 Stabilisation of the oxonium intermediate by DMF. 
2.17 Modified Mitsunobu reaction in DMF at $0{ }^{\circ} \mathrm{C}$ with $\mathrm{TMSN}_{3}$ as the nucleophile.

2.18 Modified Mitsunobu reaction in DMF at $-20{ }^{\circ} \mathrm{C}$ with $\mathrm{TMSN}_{3}$ as the 54 nucleophile.

2.19 Modified Mitsunobu reaction in DMF at $-40{ }^{\circ} \mathrm{C}$ with $\mathrm{TMSN}_{3}$ as the 54 nucleophile.

2.20 Possible mechanism for formation of azide 2.9.

2.21 Ring expansion of $\mathbf{2 . 1 2}$ generating the leaving group in situ. 56

$\begin{array}{lll}\text { 2.22 } & \text { Rydzewski's diazide synthesis. } & 59\end{array}$

2.23 Rearrangement of oxepine 2.12 with $\mathrm{TMSN}_{3}$ in TMSOTf to give 62 observed reaction products.

2.24 Fraser-Reid's Claisen approach to $C$-alkylation of glycals. 63

2.25 Tebbe-Claisen approach to formation of C-glycosides. 63

$\begin{array}{lll}\text { 2.26 Formation of Tebbe's reagent. } & 64\end{array}$

2.27 Damha and Sabatino's formation of an oxepane nucleoside. 65

2.28 Rearrangement of $\mathbf{1 . 6 1}$ by tandem Tebbe-Claisen rearrangement to 66 form 2.16.

2.29 Possible reaction mechanism during Modified Tebbe-Claisen leading 66 to observed products.

3.1 Reaction scheme for the formation of cyclopropane 3.4. 71

3.2 General method for the expansion of cyclopropane 3.4. 72

3.3 Equilibria involved in the interception of the oxonium intermediate 75 with a TMS nucleophile.

3.4 Formation of $\mathbf{3 . 1 6}$ and its ring expansion.

3.5 Formation of di-tert-butylsilyl allal cyclopropane. 82

3.6 Effect of the stereochemistry of the leaving group on the rate of 83 reaction.

4.1 Ring expansion of cyclopropane 3.16 with nucleophile 3.9. 88

4.2 Possible mechanism leading to the formation of oxepine 4.1. 88 
4.3 Possible reaction mechanism to account for the formation of oxepines 4.3 and 4.4 from 4.5.

4.4 Degradation of pectin to galacturonic acid. $\mathrm{R}$ is an oligosaccharide. 93

4.5 Ring expansion of $\mathbf{1 . 6 1}$ with TESH. 93

4.6 Formation of oxepine 4.7 from cyclopropane 3.4. 95

4.7 Possible deoxygenation mechanism leading to oxepine 4.7. 95

4.8 Formation of deuterated oxepine 4.10. 99

4.9 Possible mechanism to account for the formation of oxepines $\mathbf{4 . 8}$ and 100 4.9.

$\begin{array}{lll}\text { 5.1 } & \text { Bromine addition to an alkene. }\end{array}$

5.2 Bromine intermediate co-ordinating to the C-5 oxygen followed by 114 attack of the nucleophile

5.3 Epoxidation mechanism with a peracid

5.4 Mechanism for osmium tetraoxide dihydroxylation.

5.5 Standard hydroboration showing addition at least substituted carbon center

5.6 Possible borane intermediate formation leading to observed alcohol stereochemistry

6.1 Escher and Niclass' synthesis of oxepane 6.1

6.2 Projected reaction scheme for the total synthesis of quince oxepane

6.3 Reaction scheme for the formation of oxepine 6.5

6.4 Mono deprotection of $\mathbf{6 . 3}$ to give diol 6.6.

6.5 Formation of oxepine diol 6.7

6.6 Revised synthetic scheme using glucal as a starting point.

6.7 Grignard addition to ketone 3.20 showing preferred route of attack

6.8 Reaction scheme for installation of the side chain

6.9 Products from the oxidation/Wittig reaction.

6.10 Possible mechanism formation of the cyclohexyl side product 
$\begin{array}{lll}\text { 6.11 } & \text { Formation of oxepine 6.11 } & 135\end{array}$

6.12 Formation of sidechain diene and xanthate 6.15 136

6.13 Possible radical isomers formed during Barton-McCombie 137 deoxygenation

6.14 Revised synthetic scheme for formation of the sidechain 139

$\begin{array}{lll}\text { 6.15 Diol clevage by BAIB } & 140\end{array}$

6.16 Possible reaction paths for formation of the products 6.9 and $\mathbf{6 . 1 0}$ in $\quad 140$ scheme 6.9

$\begin{array}{lll}\text { 6.17 } & \text { Mechanism of formation of the cyclohexyl diol 6.7 } & 141\end{array}$

7.1 Possible alternative reaction scheme towards the formation of oxepine 146 6.1 


\section{List of Figures}

1.1 Generalised equation for photosynthesis 2

1.2 Enantiomers of glyceradehyde 4

1.3 Fischer projections of enantiomers of glycereldehyde 4

1.4 Fischer projections of the tetraoses 5

$\begin{array}{lll}\text { 1.5 } & \text { Examples of monosaccharides } & 7\end{array}$

$\begin{array}{lll}1.6 & \text { Examples of disaccharides } & 7\end{array}$

1.7 The oligosaccharide component of Globo-H $1.6 \quad 8$

1.8 Linkages of two example disaccharides 9

1.9 The structures of cellulose and starch 9

$\begin{array}{lll}\text { 1.10 Examples of glycals } & 11\end{array}$

$\begin{array}{lll}\text { 1.11 Danishefsky's Ferrier rearrangement starting materials } & 14\end{array}$

1.12 Das and co-workers $\mathrm{InCl}_{3}$ catalysed rearrangements under microwave 15 irradiation

$\begin{array}{lll}1.13 & \text { Allylic direction of Simmons-Smith cyclopropanation showing } & 17\end{array}$ 'butterfly' intermediate state with a dimeric zinc species delivering the carbene syn to the allylic alcohol

1.14 Complex cyclopropanes synthesised using Simmons-Smith 20 methodology

1.15 Naturally occurring compounds containing seven-membered oxacycles 28

1.16 Numbering conventions used in this thesis for the purposes of NMR 39

2.1 Possible structure of product 2.7b from triflation of $\mathbf{2 . 3} 46$

2.2 Proposed structure of oxepine 2.13 58 
2.3 Substructure of middle $\mathrm{R}_{\mathrm{F}}$ compound generated from ring expansion with leaving group generated in situ

2.4 Probable structure of diazide $\mathbf{2 . 1 4}$

2.5 Proposed conformation of diazide $\mathbf{2 . 1 4}$ showing interaction between positively charged nitrogen and the free alcohol

2.6 Proposed structure for the nitrile $\mathbf{2 . 1 5}$

2.7 Structure of AZT

3.1 Cyclopropanated glucal system 1.61.

3.2 Cyclopropanated galactal system 3.4.

$\begin{array}{lll}\text { 3.3 } & \text { Glucal derived oxonium intermediate } & 70\end{array}$

$\begin{array}{lll}\text { 3.4 Galactal derived oxonium intermediate } & 70\end{array}$

$\begin{array}{lll}\text { 3.5 } & \text { By-product 3.2 from formation of silyl ether 3.1 }\end{array}$

3.6 Selected NOE enhancements which were used to assign the 73 stereochemistry of the major 3.5a and minor 3.5b isomers

3.7 NOE enhancement used to assign the stereochemistry of the major epimer of $\mathbf{3 . 8}$

3.8 Formation of oxonium intermediate with $\sigma^{*}$ orbitals shown in red

3.9 TMS nucleophiles to investigate steric constraints of the reaction

3.10 NOE enhancements assigning the structure of the major epimer 3.11 78

3.11 Mechanism for the formation of the oxonium intermediate from cyclopropanated glucal $\mathbf{1 . 6 1}$

3.12 Mechanism for the formation of the oxonium intermediate from cyclopropanated allal $\mathbf{3 . 2 2}$

3.13 Diaxial interactions in Ferrier rearrangement of glycals and nucleophiles 3.9 or 3.10 
4.1 Product 4.2 from the ring expansion of cyclopropane 3.16 with nucleophile 3.10

4.2 Large substructure from product of TMSallyl reaction with cyclopropane $\mathbf{3 . 1 6}$

4.3 Small substructure from product of TMSallyl reaction with cyclopropane $\mathbf{3 . 1 6}$

4.4 Structure of diallyl oxepine $\mathbf{4 . 3}$ numbered for the assignment in the text 91

4.5 Products from the reaction of TMSallyl with cyclopropane 3.16 91

4.6 Deuterated compound oxepine 4.8 from TESD reaction 97

$\begin{array}{lll}\text { 4.7 } & \text { Minor product 4.9 from ring expansion with TESD }\end{array}$

$\begin{array}{lll}\text { 5.1 Known conformations of septanose rings } & 102\end{array}$

5.2 Oxepine $\mathbf{3 . 1 2}$ for elaboration showing ring-numbering system for used 103 for NMR analysis

5.3 Structure of peloruside A

$\begin{array}{lll}\text { 5.4 Oxepane derived from } \mathbf{3 . 1 2} \text { overlayed on structure of peloruside A } & 104\end{array}$

5.5 Minor isomer 5.2 from bromination showing NOE enhancements and 106 ${ }^{1} \mathrm{H}$ axial-axial interactions

5.6 $\quad{ }^{1} \mathrm{H}$ NMR spectrum of dibromide 5.1 in $\mathrm{CDCl}_{3}(500 \mathrm{MHz})$ showing $\quad 107$ congested region between 4.2 and $4.0 \mathrm{ppm}$

5.7 HSQC spectrum of dibromide $\mathbf{5 . 1}$ in $\mathrm{CDCl}_{3}$ showing overlapping proton 108 signals between 4.2 and $4.0 \mathrm{ppm}$

5.8 Major isomer 5.1 from bromination showing NOE enhancements and 109 anti ${ }^{1} \mathrm{H}$ axial-axial interactions

5.9 Diol 5.3

5.10 ${ }^{1} \mathrm{H}$ NMR spectra of dibromide $\mathbf{5 . 3}$ in $\mathrm{CDCl}_{3}(500 \mathrm{MHz})$ demonstrating 111 resolution of the previously congested area between 4.2 and 4.0

5.11 HSQC spectrum of dibromide 5.3 in $\mathrm{CDCl}_{3}$ showing resolution of overlapping proton signals 
5.12 Dibromide 5.3 showing NOE enhancements and ${ }^{1} \mathrm{H}$ axial-axial interactions

5.13 Major isomer 5.10 from acetylation of bromohydrin $\mathbf{5 . 4}$ showing NOE enhancements and ${ }^{1} \mathrm{H}$ axial-axial interactions

5.14 Structure of epoxide $\mathbf{5 . 5}$ showing NOE enhancements and ${ }^{1} \mathrm{H}$ axial-axial 116 interactions

5.15 Structure of actylated azide 5.11 showing NOE enhancements and ${ }^{1} \mathrm{H}$ axial-axial interactions

5.16 Stereochemistry of the azide addition to epoxide $\mathbf{5 . 5}$

5.17 Structure of diacetate $\mathbf{5 . 1 2}$ showing NOE enhancements and ${ }^{\mathrm{l}} \mathrm{H}$ axialaxial interactions

5.18 Structure of monoacetate $\mathbf{5 . 1 3}$ showing NOE enhancements and ${ }^{1} \mathrm{H}$ axial-axial interactions

5.19 Alcohol 5.9 from dibal reduction of the oxepine ester 3.12

6.1 Cydonia obolonga

6.2 Seven membered ring ethers isolated from quince brandy

6.3 Possible $\mathrm{H}$ bonding in diol 6.7 preventing oxidation of primary alcohol

6.4 Product 6.13 formed from Wittig reaction with 6.12

6.5 Structure of cyclohexyl pyrene $\mathbf{6 . 1 7}$

6.6 Major and minor products from reaction of 6.7 with TESD

6.7 NOE enhancements for minor product diol 6.19 


\section{List of Tables}

1.1 Cyclopropanations of protected glucals using the Furukawa 19 modification of the Simmons-Smith reaction cyclopropanation

1.2 Results of cyclopropation of glycals with diazides 22

1.3 Results of cyclopropanations using Nagarajan's method 23

$\begin{array}{lll}\text { 1.4 Cyclopropane expansions using TMSOTf } & 37\end{array}$

2.1 Reaction of cyclopropane $\mathbf{2 . 3}$ under modified Mitsunobu conditions $\quad 55$

$\begin{array}{lll}\text { 3.1 } & \text { Results of initial ring expansion reactions of } \mathbf{3 . 4} & 73\end{array}$

3.2 Ring expansions of cyclopropane 3.4 with nucleophiles 3.9 and $3.10 \quad 77$

$\begin{array}{lll}\text { 3.3 Results of ring expansions of cyclopropane 3.16 } & 80\end{array}$

3.4 Summary of ring expansions of cyclopropanes $\mathbf{3 . 4}$ and $\mathbf{3 . 1 6}$ verses $\quad 84$ glycal rearrangements

5.1 Results of elaborations of oxepine $\mathbf{3 . 1 2}$ 


\section{List of Abbreviations}

\begin{tabular}{|c|c|}
\hline Ac & Acetate \\
\hline $\mathrm{Ac}_{2} \mathrm{O}$ & Acetic anhydride \\
\hline $\mathrm{AgOTf}$ & Silver triflate \\
\hline AIBN & 2,2'-Azobis(2-methylpropionitrile) \\
\hline AT & Ambient temperature \\
\hline 9-BBN & 9-Borabicyclo[3.3.1] nonane \\
\hline BAIB & [Bis(acetoxy)iodo]benzene \\
\hline $\mathrm{Bn}$ & Benzyl \\
\hline BuLi & Butyl lithium \\
\hline$\left(\mathrm{Bu}_{3} \mathrm{Sn}\right)_{2} \mathrm{O}$ & Bis(tributyltin) oxide \\
\hline $\mathrm{Cp}$ & Cyclopentadiene \\
\hline m-CPBA & m-Chloroperoxybenzoic acid \\
\hline $\mathrm{Da}$ & Daltons \\
\hline DEAD & Diethylazodicarboxylate \\
\hline DMAP & 4- $N, N$-Dimethylaminopyridine \\
\hline DMF & Dimethylformamide \\
\hline DMPU & 1,3-Dimethyl-3,4,5,6-tetrahydro-2(1H)-pyrimidinone \\
\hline DMSO & Dimethylsulphoxide \\
\hline ds & Diastereoselectivity \\
\hline $\mathrm{E}$ & Electrophile \\
\hline eq & Equivalents \\
\hline ES & Electrospray \\
\hline $\mathrm{Et}$ & Ethyl \\
\hline $\mathrm{Et}_{2} \mathrm{O}$ & Diethylether \\
\hline EtOAc & EtOAc \\
\hline
\end{tabular}




\begin{tabular}{|c|c|}
\hline $\mathrm{EtOH}$ & Ethanol \\
\hline $\mathrm{Et}_{2} \mathrm{Zn}$ & Diethylzinc \\
\hline GC & Gas chromatography \\
\hline $\mathrm{h}$ & Hour \\
\hline HRMS & High resolution mass spectrometry \\
\hline IR & Infrared spectrometer \\
\hline LA & Lewis acid \\
\hline LG & Leaving group \\
\hline LAH & Lithium aluminium hydride \\
\hline $\mathrm{Me}$ & Methyl \\
\hline $\mathrm{MeCN}$ & Acetonitrile \\
\hline $\mathrm{Mp}$ & Melting point \\
\hline Ms & Mesylate \\
\hline NBS & N-Bromosuccinamide \\
\hline NIS & N-Iodosuccinamide \\
\hline NMO & $\mathrm{N}$-methylmorpholine-N-oxide \\
\hline NMR & Nuclear magnetic resonance \\
\hline NOE & Nuclear Overhauser effect \\
\hline $\mathrm{Nu}$ & Nucleophile \\
\hline $\mathrm{O} / \mathrm{N}$ & Overnight \\
\hline PCC & Pyridinium chlorochromate \\
\hline $\mathrm{PhCH}_{3}$ & Toluene \\
\hline $\mathrm{PPh}_{3}$ & Triphenylphosphine \\
\hline py & Pyridine \\
\hline $\mathrm{RCM}$ & Ring closing metathesis \\
\hline TBAF & Tetrabutylammonium fluoride \\
\hline
\end{tabular}




$\begin{array}{ll}\text { TBDPS } & \text { tert-Butyldiphenylsilyl } \\ \text { TBS } & \text { tert-Butyl silyl } \\ \text { TEA } & \text { Triethylamine } \\ \text { TES } & \text { Triethyl silyl } \\ \text { TESH } & \text { Triethyl silyl hydride } \\ \text { Tf } & \text { Triflate (trifluoromethanesulfonyl) } \\ \text { TfO } 2 & \text { Triflic anhydride } \\ \text { THF } & \text { Tetrahydrofuran } \\ \text { TIPS } & \text { Triisopropylsilyl } \\ \text { TLC } & \text { Thin layer chromatography } \\ \text { TMS } & \text { Trimethylsilyl } \\ \text { TMSOTf } & \text { Trimethylsilyl triflate } \\ \text { TOCSY } & \text { Total correlation spectroscopy } \\ \text { UV } & \text { Ultraviolet }\end{array}$




\section{Chapter 1: Introduction}

The author has been both praised and abused for using carbohydrate derivatives "to do organic chemistry”. One friend allowed that there are aspects of sugar chemistry which deserve the attention of competent chemists, while another expressed amazement that we know how to use a drybox since sugars are water soluble. A Nobel Laureate known to the author declared that the stabilisation of the anomeric cation by the ring oxygen constitutes half of sugar chemistry. A graduate student (from another area) was amazed to find that many of the fabled mysteries of the hexoses garnered during that fateful two weeks of Org. Chem. II disappeared once he drew the structures properly. An eminent chemist wants to use sugars; but he finds the names annoying. ${ }^{1}$

These views, expressed by Bert Fraser-Reid in July 1974 sum up the position that carbohydrate chemistry held 40 years ago (around the time the author of this thesis was born). Since then carbohydrate chemistry has moved from a fringe area of organic chemistry investigated by a few dedicated researchers, to one of the central fields of current chemical investigation due to the vital and diverse biological importance of carbohydrates and their increasing utility in chemical synthesis and industry.

\subsection{An introduction to carbohydrates}

Historically, the name carbohydrate comes from the formula of this class of compounds which is $\mathrm{C}_{\mathrm{n}}\left(\mathrm{H}_{2} \mathrm{O}\right)_{\mathrm{n}}$, quite literally a hydrate of carbon. This generic term covers a large well-defined group of organic compounds, which are mostly aliphatic polyhydroxy aldehydes and ketones. It is also used to cover compounds obtained from their reduction and oxidation, as well as replacement of one or more hydroxyl groups with 
either a hydrogen atom, a heteroatom or other functional group. Carbohydrates are synthesised in green plants by a variety of biochemical pathways collectively known as photosynthesis. This process uses solar energy to 'fix' carbon dioxide in a form that can then be metabolised by plants to generate energy, with $\mathrm{O}_{2}$ as a reaction by-product (Figure 1.1). ${ }^{2,3}$ Without this process life on earth as we know it would not be possible.

$$
x \mathrm{CO}_{2}+y \mathrm{H}_{2} \mathrm{O} \stackrel{\mathrm{h} v}{\longrightarrow} \mathrm{C} x\left(\mathrm{H}_{2} \mathrm{O}\right) y+x \mathrm{O}_{2}
$$

Figure 1.1. Generalised equation for photosynthesis.

Carbohydrates are an abundant and renewable source of feedstocks for modern chemical research and industry. They are far cheaper and in many cases have advantages over petroleum-based products, providing a rich source of functionality, chirality and structural variety. ${ }^{4}$ To give an impression of how important this market is, in 2005 the United States used $70 \%$ of its oil in the transportation field. This had a pretax value of $\$ 385$ billion dollars. In contrast only $3.4 \%$ was used in the petrochemical industry (plastics, cosmetics, detergents and paints etc). However this had a pre-tax value of $\$ 375$ billion dollars. ${ }^{5}$ With the price of oil only set to rise as the reserves slowly and inexorably run out the stage is set for another source of feedstocks to replace it.

The cornerstone of the chemistry of carbohydrates was laid in the 1880s by Emil Fischer, one of the true giants of organic chemistry. ${ }^{6}$ In a series of experiments over a seven year period he elucidated the structures and stereochemistry of all the basic carbohydrates, or monosaccharides as they are more correctly known. ${ }^{7}$ This monumental achievement saw him awarded the second Nobel prize in chemistry in $1902 .^{8}$ 
Despite these major advances, subsequent progress in the field was slow. Fischer himself alluded to these problems in a letter to his mentor Baeyer in January 1889.

"Unfortunately, the experimental difficulties in this group are so great, that a single experiment takes more time in weeks than other classes of compounds take in hours, so only very rarely a student is found who can be used for this work."9

Many of the problems with working with these compounds were caused in large part by their abundance of hydrophilic groups. Characterisation was mostly by derivatisation, which made further use of the compounds rather difficult. Nonetheless a small group of dedicated researchers persisted in working in the field. These workers laid a solid foundation for those who would come after them. With the development of $\mathrm{NMR}^{10}$ and improved chromatography techniques, the characterisation of carbohydrate compounds became greatly simplified. NMR in particular became a very important technique as carbohydrates by their nature give spectra that, while complex, are extremely well resolved. Indeed, carbohydrate chemists were innovators in the field of $1 \mathrm{D}$ and $2 \mathrm{D}$ NMR, adopting and applying new pulse sequences and experiment types long before they became popular in more mainstream organic chemistry fields. In the 1970s came the discovery that sugars played a central role in complex biological interactions particularly those at the cell surface. ${ }^{11,12}$ Interest in the field has grown exponentially since this time. 


\subsection{Conventions for the description of carbohydrate structure and conformers $^{13}$}

The simplest monosaccharide possessing a chiral center is the compound glyceraldehyde, which contains three carbon atoms and is also known as a triose. This can exist as two enatiomers (Figure 1.2).

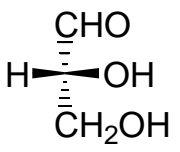

$(R)$-glyceraldehyde

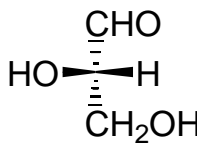

(S)-glyceraldehyde

Figure 1.2. Enantiomers of glyceraldehyde.

However, before the absolute configurations of carbohydrates were known, Fisher proposed that the enantiomers should be classified by their optical rotations, D- for dextrorotatory or L- for levorotatory. Thus the enantiomers of glyceraldehyde were drawn and named by Fischer as shown in Figure 1.3. This was further extended by Rosanoff to cover a great many other compounds using the glyceraldehydes as the base. $^{14,15}$<smiles>O=CC(O)CO</smiles>

D-glyceraldehyde<smiles>CC(O)C=O</smiles>

L-glyceraldehyde

Figure 1.3. Fischer projections of enantiomers of glyceraldehyde.

The Fischer projection is drawn with the aldehyde at the top of the carbon backbone and the primary hydroxyl at the bottom. The D- and L- terms are used to refer to the orientation of the alcohol on the chiral carbon. The carbohydrates with four carbons are 
called tetroses and have two chiral centers. This gives two enantiomeric pairs as shown in Figure 1.4.<smiles>O=CC(O)C(CO)CO</smiles>

D-erythrose<smiles>O=CC(O)C(O)CO</smiles>

L-erythrose<smiles>O=CC(O)C(O)CO</smiles>

D-threose<smiles>O=CC(O)C(O)CO</smiles>

L-threose

Figure 1.4. Fischer projections of the tetroses.

In this case the D- and L- conventions refer to the chiral carbon furtherest from the aldehyde. Continuing the series there are pentoses with three chiral centers giving four pairs of enantiomers and hexoses with four chiral centers giving eight pairs of enantiomers. Carbohydrates with larger numbers of carbon atoms are also known. These are septanoses (seven carbons), octoses (eight carbons), nonoses (nine carbons) and decoses (ten carbons). Pentoses and hexoses exist naturally as cyclic systems. This occurs when the carbonyl reacts with one of the hydroxyls in the molecule and forms a cyclic hemiacetal that is more stable than the open chain form. The cyclic form of the pentoses are called furanoses (with a five-membered ring). The hexoses can exist as furanoses or pyranoses (with a six membered ring) depending on whether the ring is formed with the C-4 or C-5 hydroxyl (Figure 1.5).

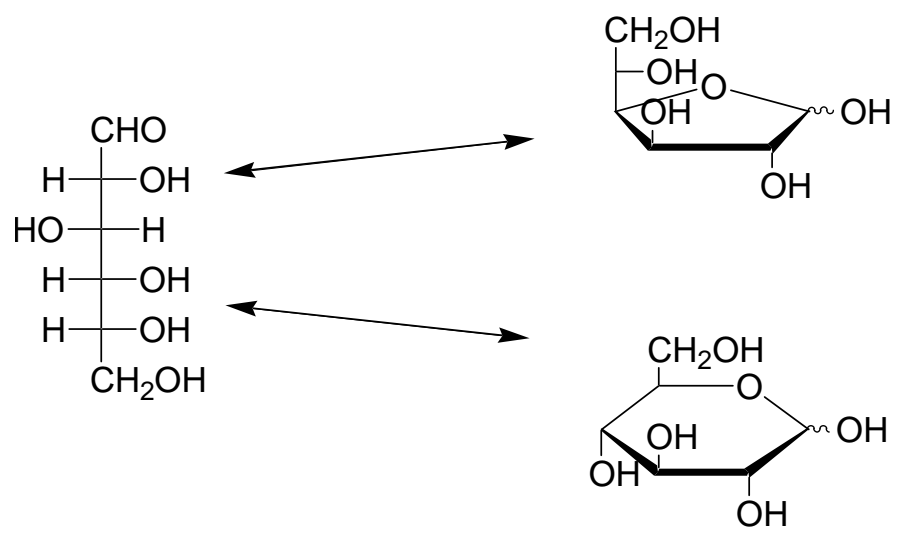

Scheme 1.1. Furanose and pyranose forms of D-glucose. 
The 3D structures of the cyclic forms are represented here in Haworth projections.

Carbohydrates with more than six carbons form pyranose rings with an exocyclic carbon chain and are known as extended chain carbohydrates. Furanose and pyranose rings exist as two different isomers, known as anomers. The carbon at this new center is known as the anomeric center. This depends on the orientation of the hemiacetal formed between the hydroxyl and the aldehyde center. If the new $\mathrm{OH}$ is formed on the same side in the Fischer projection as the the oxygen involved in the hemiacetal link then it is the $\alpha$ anomer. If it is on the opposite side then it is the $\beta$ anomer. For the Dcarbohydrates, this translates to a Haworth projection where the anomeric hydroxyl is down for the $\alpha$ and up for the $\beta$ anomer (Scheme 1.2). It is common for the lower face of a carbohydrate to be refered to as the $\alpha$ face, and the upper face the $\beta$ face.

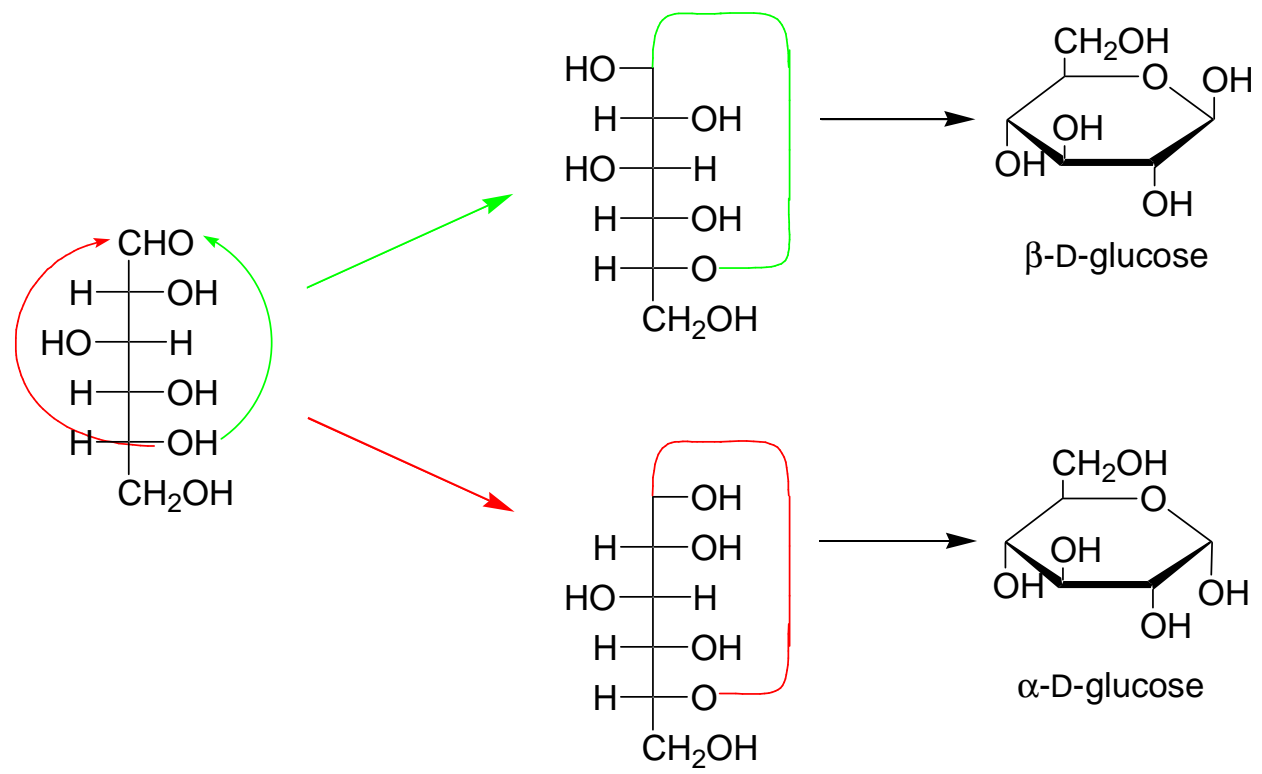

Scheme 1.2. Hemiacetal formation leading to $\alpha$ and $\beta$ anomeric forms.

\subsection{Glycosidic linkages}

Naturally occurring monosaccharides are the simplest sugars consisting of just one base saccharide unit of between three and six carbons, and are not hydrolysed to simpler compounds under acid hydrolysis. Common examples are glucose $\mathbf{1 . 1}$ (grape sugar), 
galactose 1.2 and fructose 1.3 (fruit sugar) shown in Figure 1.5. These sugars are broken down by enzymes through various biochemical pathways and used as energy.

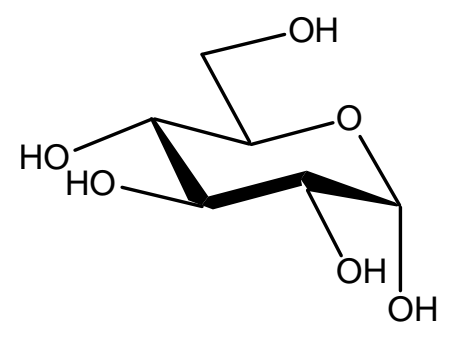

$\alpha$-D-Glucose 1.1

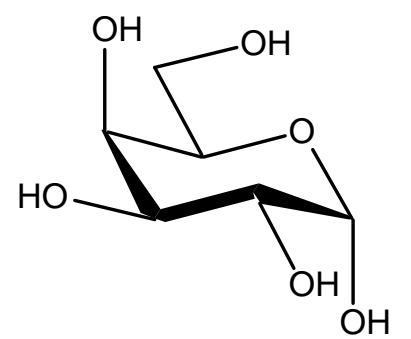

$\alpha$-D-Galactose 1.2

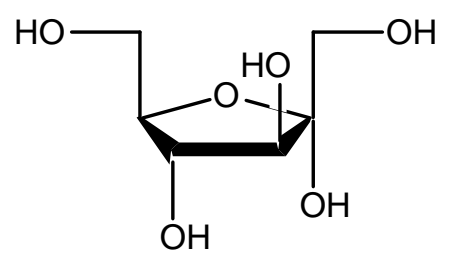

$\alpha$-D-Fructose $\mathbf{1 . 3}$

Figure 1.5. Examples of monosaccharides.

When two monosaccharide units are linked together by an $O$-glycosidic linkage they are called a disaccharide. Common examples are sucrose $\mathbf{1 . 4}$ (cane sugar) and lactose $\mathbf{1 . 5}$ (milk sugar) illustrated in Figure 1.6.

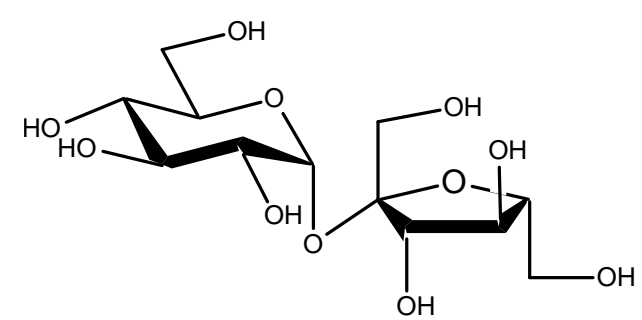

Sucrose 1.4

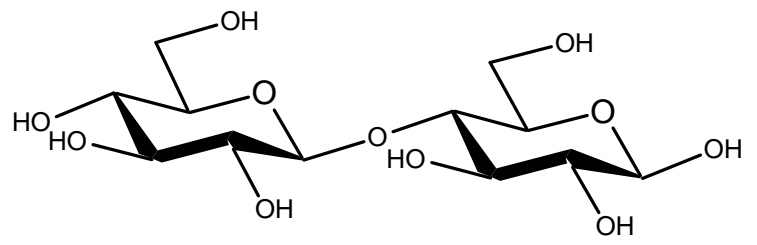

Lactose $\mathbf{1 . 5}$

Figure 1.6. Examples of disaccharides.

Three or more monosaccharide's connected by glycosidic linkages are termed oligosaccharides. The role of these in cells is not generally as a form of energy storage. They are commonly found on the surface of cells. These generally consist of fewer than 20 monosaccharide units, and are used in a vast array of biological signalling systems. A simple example of these oligosaccharides is found in Globo-H 1.6. ${ }^{16}$ This is a cellsurface glycosphingolipid, which occurs in a number of tumours including breast, prostate and ovary (Figure 1.7). 


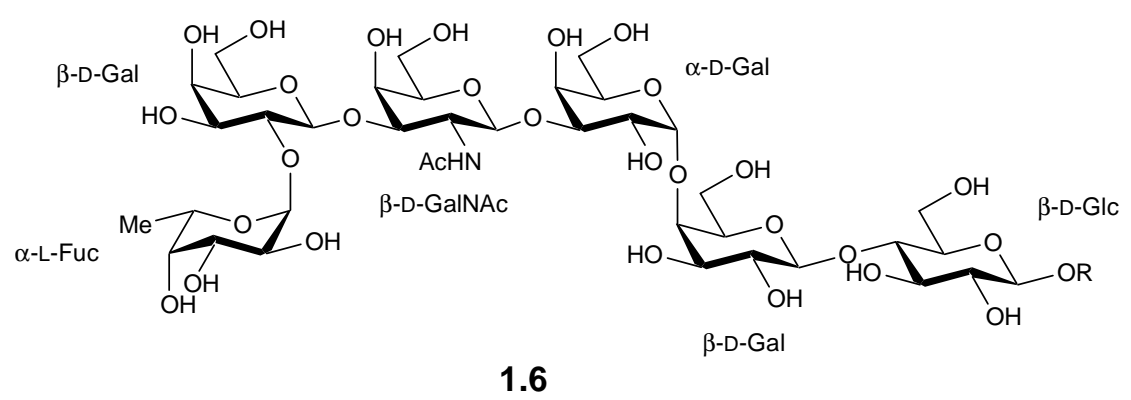

Figure 1.7. The oligosaccharide component of Globo-H 1.6.

Cyclic oligosaccharides are also known, more commonly called cyclodextrins. These consist of most commonly 6-8 glucose residues linked in a $(1 \rightarrow 4)$ fashion, but can contain upwards of 30 glucose residues. These compounds are used as energy storage by some bacteria that have an enzyme that can convert the rings back into glucose. This process is essential as bacteria lacking the enzyme are not viable in the environment and die out.

Finally, there are the polysaccharides; these large oligosaccharides consist of upwards of 500 monosaccharides. They exist in structural (e.g. cellulose and chitin) and energy storage (e.g. starch) forms. In all these polysaccharides the constituent monosaccharides are linked to each other by glycosidic bonds. The most prevalent in nature are $O-, S$-, and $N$ - linkages. These are formed between any two monosaccharide functional groups. This makes polysaccharides the most structurally complex biopolymer. For example, only one dipeptide can be formed from two identical amino acids. However with two identical hexoses the number of different possible disaccharides is 11 with $O$-linked glycosides. ${ }^{17}$ This is because not only can there be glycosidic bonds formed between any of the different carbon atoms, but also these linkages can be $\alpha$ or $\beta$. Figure 1.8 shows examples of two different disaccharides, maltose and lactose. 


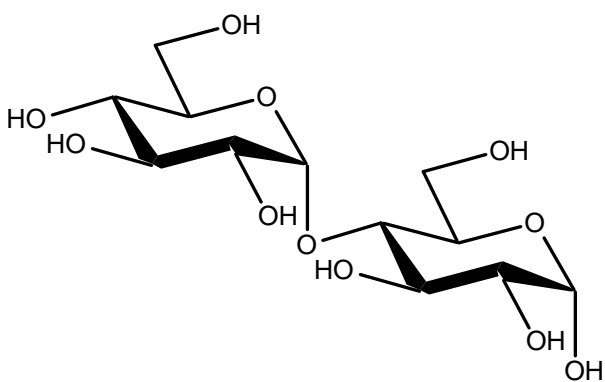

Maltose

$\alpha-(1-4)$ linkage

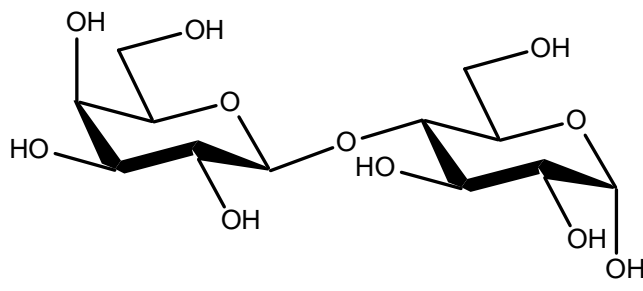

Lactose

$\beta$-(1-4) linkage

Figure 1.8. Linkages of two example disaccharides.

The major structural difference between these two disaccharides is the orientation of the glycosidic linkage. Maltose with its $\alpha(1 \rightarrow 4)$ glycosidic linkage can be processed by the yeast Saccharomyces cerevisiae (brewers yeast) to ethanol and $\mathrm{CO}_{2}$. However, S. cerevisiae cannot not process lactose in the same way as it lacks the enzyme to break the $\beta(1 \rightarrow 4)$ glycosidic linkage. This can be used to the brewer's advantage as lactose can be added into the wort to increase the level of final sweetness. The biological importance of anomerism is also illustrated in the structures of cellulose and starch. Cellulose is made up of repeating $\beta(1 \rightarrow 4)$-D-glucose units. It makes up the cell walls of green plants. Starch is made up of repeating $\alpha(1 \rightarrow 4)$-D-glucose units and is used by plant cells to store glucose in an easily retrievable form (Figure 1.9).

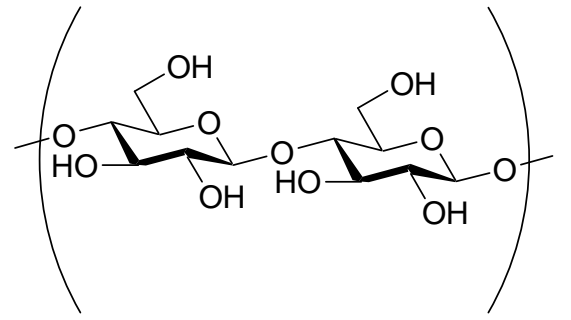

Cellulose

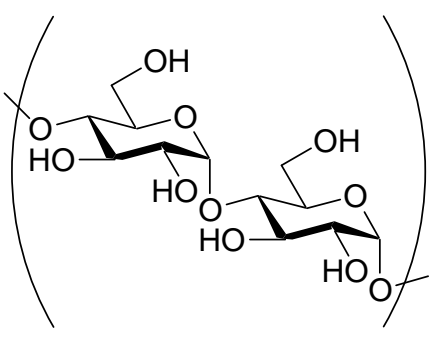

Starch

Figure 1.9. The structures of cellulose and starch.

Again, while starch can be broken down by enzymes present in higher animals, only 
bacteria have enzymes to break down cellulose. Ruminants that can digest cellulose do so by having the relevant bacteria present in their gut flora.

A major problem with biologically-derived oligosaccharides is that they are extremely difficult to extract from natural systems in adequate yields and purity. Thus much research has gone into the selective formation of the different types of glycosidic bonds and the subsequent formation of the target oligosaccharides. There are several commonly used methods to form these glycosides. The first involves the activation of a leaving group on a glycosyl donor by an electrophilic promoter. This is followed by nucleophilic attack of the glycosyl acceptor on the anomeric carbon of the glycosyl donor (Scheme 1.3).

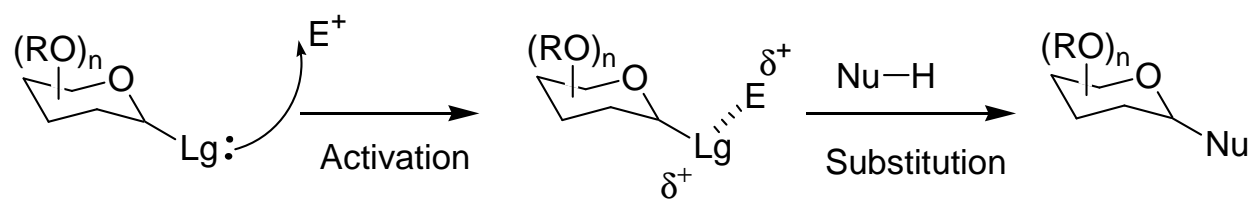

Scheme 1.3. Glycosylation by a glycosyl donor.

This reaction tolerates a wide variety of acceptors depending on the reaction conditions used. With these tools it has been possible to synthesise a wide variety of oligosaccharides. As well as the naturally occurring targets it is also possible to synthesise non-natural oligosaccharides in order to probe biological functions or attempt to synthesise vaccines against various infections and diseases.

These glycosylations are limited to $O-, N$ - and $S$ - linked glycosides. Another class of naturally occurring glycosides are those where the linker to the anomeric carbon is another carbon atom. These are unsurprisingly known as $C$-glycosides. It is only in the last 40 years that there has been increased interest in this type of glycoside. This has been due to the discovery of biologically active molecules containing this linkage. 
Another driver has been the increased use of carbohydrates in organic synthesis as chiral building blocks and related development of new synthetic methodologies to better employ these chirons. A more recent field of interest has been use of these compounds as analogs of cell surface glycans and as inhibitors of carbohydrate processing enzymes. The major difference between $C$ - and other glycosides is their inherent stability to a wide range of reaction conditions, including cellular metabolism, at the anomeric center due to the presence of the $\mathrm{C}-\mathrm{C}$ bond.

As with the previously mentioned glycosides, $C$-glycosides can be formed by attack of a nucleophile on a glycosyl donor with a suitable leaving group. It is possible to perform any chemistry that results in the formation of a $\mathrm{C}-\mathrm{C}$ bond. This includes the use of organometalic reagents such as Grignard reagents and stannane chemistry. It has even proved possible to do Wittig type reactions at the anomeric centre. The most synthetically useful group of glycosyl donors are unsaturated monosaccharides that contain an alkene bond in the molecule. Of particular note are the unsaturated compounds commonly known as glycals. These compounds are characterised by having an alkene present between $\mathrm{C}-1$ and $\mathrm{C}-2$ which makes these compounds analogs of vinyl ethers (Figure 1.10). Reactions with these compounds can generate products with excellent control over regio- and stereo-selectivity.<smiles>OC[C@H]1OC=C[C@@H](O)[C@@H]1O</smiles>

Glucal<smiles>OC[C@H]1OC=C[C@@H](O)[C@@H]1O</smiles>

Galactal

Figure 1.10. Examples of glycals. 
The use of glycals as glycosyl donors is well known. As seen in Scheme 1.3, the glycal donor is also activated by an electrophilic promoter, followed by coupling with a nucleophilic glycosyl acceptor. However, in this reaction it is also possible to introduce another group $\mathrm{Z}$ at the $\mathrm{C}-2$ position at the same time as the formation of the anomeric bond (Scheme 1.4). These $\mathrm{Z}$ groups are most commonly azides, acids, amines or alcohols.

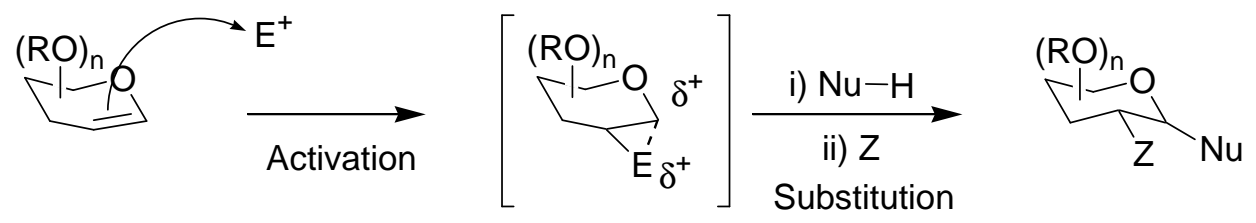

Scheme 1.4. Glycosylation involving a glycal donor.

One of the more chemical useful reactions involving glycals is the Ferrier rearrangement. ${ }^{18-21}$ This adds a nucleophile to a glycal with a moderate to high stereoand regioselectivity at the $\mathrm{C}-1$ position, promoted by loss of a leaving group from $\mathrm{C}-3$ and the subsequent migration of the alkene to between C-2 and C-3 (Scheme 1.5).

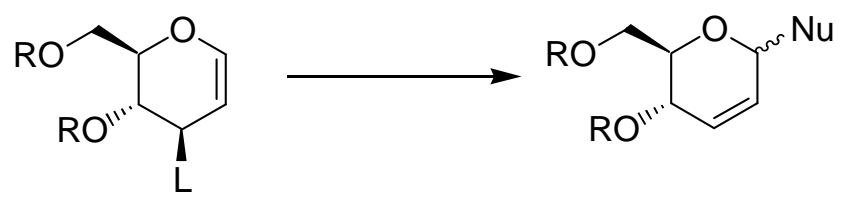

Scheme 1.5. Ferrier glycal rearrangement.

It is most commonly proposed that this reaction proceeds through an oxonium intermediate; although results suggest that other mechanisms may also contribute to the transformation or compete to provide alternate products. These mechanisms are summarised in Scheme 1.6. With acetate as the leaving group at the C-3 position, there exists the possibility of A) attack by a nucleophile at C-1, or B) rearrangement of the leaving group to $\mathrm{C}-1$ followed by nucleophilic attack at C-3. 
A)

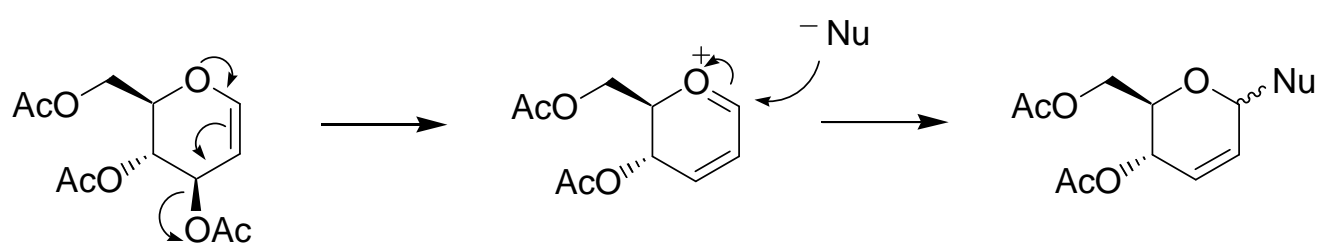

B)<smiles>CC(=O)OCC1OC2CCCC(OC(C)=O)C1C2OC(C)=O</smiles><smiles>CC(=O)OC[C@H]1OC=C[C@H](N)[C@H]1OC(C)=O</smiles>

Scheme 1.6 Possible mechanisms involved in Ferrier rearrangements

Depending on the conditions and nucleophile involved, the result of the reaction can be tuned to afford either C-1 or C-3 attack. The stereochemical outcome of reaction path A can also be controlled depending on nucleophile and conditions. Regioselectivity is mixed in reactions involving heteronucleophiles that can generate either $\mathrm{C}-1$ or $\mathrm{C}-3$ substituted glycals. Carbon nucleophiles demonstrate regioselectivity for the C-1 position. All nucleophilic attack at the C-1 position favours $\alpha$ over $\beta$ stereoselectivity.

Danishefsky and co-workers reported investigations into Ferrier-type reactions generating C-1 carbon-substituted pyranose derivatives from glycal starting materials. ${ }^{22}$ Treatment of triacetyl glucal 1.7 with TMSallyl and $\mathrm{TiCl}_{4}$ gave the allylated product $\mathbf{1 . 8}$ in $85 \%$ yield and $16: 1 \alpha / \beta$ stereoselectivity (Scheme 1.7 ).

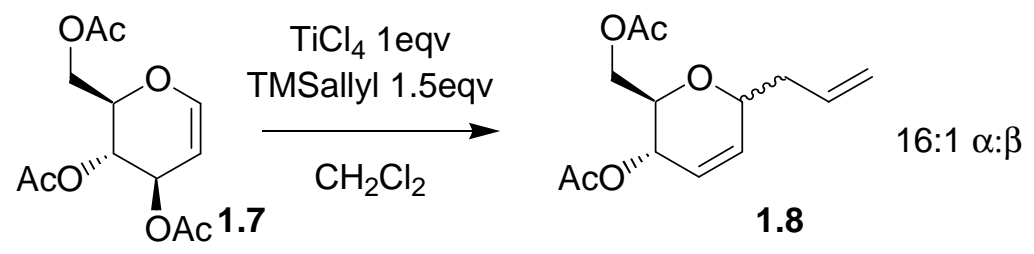

Scheme 1.7. Ferrier rearrangement of tri- $O$-acetyl-D-glucal with TMS allyl. 
Results were also reported comparing the selectivities of glucal, galactal and allal species in the Ferrier rearrangement. ${ }^{22}$ Reaction of D-galactal triacetate $\mathbf{1 . 9}$ with TMSallyl and $\mathrm{TiCl}_{4}$ in $\mathrm{CH}_{2} \mathrm{Cl}_{2}$ at $-78{ }^{\circ} \mathrm{C}$ gave $\mathbf{1 . 8}$ as a 30:1 $\alpha: \beta$ mix of epimers in $93 \%$ yield. D-Allal triacetate $\mathbf{1 . 1 0}$ gave $\mathbf{1 . 8}$ in a $95 \%$ yield but only in $6: 1 \alpha: \beta$ mix. The starting materials and product ratios are shown in Figure 1.11. No comment was made on the reaction times for these rearrangements.

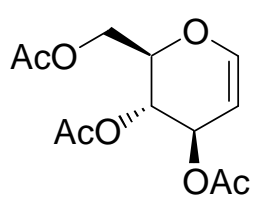

1.7

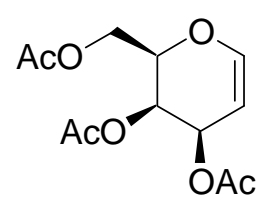

1.9<smiles></smiles>

1.10

$16: 1 \alpha: \beta$

$30: 1 \alpha: \beta$

$6: 1 \alpha: \beta$

Figure 1.11. Danishefsky's Ferrier rearrangement starting materials.

The authors propose that the high selectivity is due to a combination of axial attack by the carbon nucleophile and anti $\mathrm{S}_{\mathrm{N}} 2$ displacement of the acetate leaving group. The allal selectivity is lower due to the syn orientation of the leaving group to the approaching nucleophile.

More recently Hanna and Wlodyka reported the similar allylation of tri- $O$-acetyl-Dgalactal using $\mathrm{BF}_{3} \cdot \mathrm{Et}_{2} \mathrm{O}$ and TMSallyl at $-50{ }^{\circ} \mathrm{C}$ which gave the $\alpha$ isomer exclusively in $97 \%$ yield. $^{23}$

Das and coworkers reported on the microwave assisted Ferrier rearrangement on triacetyl glucal and triacetyl galactal using $\mathrm{InCl}_{3}$ as the catalyst. ${ }^{24}$ Their results were an improvement on Danishefsky's in terms of both yield and selectivity. Reaction of Dgalactal triacetate 1.9 with TMSallyl and $\mathrm{InCl}_{3}$ in $\mathrm{MeCN}$ under microwave irradiation gave $\mathbf{1 . 8}$ as a 99:1 $\alpha: \beta$ mix of epimers in $90 \%$ yield in only 40 seconds. D-glucal 
triacetate $\mathbf{1 . 7}$ gave $\mathbf{1 . 8}$ in a $95 \%$ yield as a 19:1 $\alpha: \beta$ mix of epimers in 30 seconds. More interesting was the result with 3,4-di-O-acetyl-ribal 1.11 which gave a selectivity of 19:1 $\alpha: \beta$ in $89 \%$ yield. This result reveals the effect of the C-5 substituent on the selectivity of the rearrangement. The starting materials and product ratios are shown in Figure 1.12.<smiles>CC(=O)OC[C@H]1OC=C[C@@H](OC(C)=O)[C@@H]1OC(C)=O</smiles>

1.7<smiles>CC(=O)OC[C@H]1OC=C[C@@H](OC(C)=O)[C@H]1OC(C)=O</smiles>

1.9<smiles>CC(=O)O[C@H]1C=COC[C@H]1OC(C)=O</smiles>

1.11

$19: 1 \alpha: \beta$ 99:1 $\alpha: \beta$

$19: 1 \alpha: \beta$

Figure 1.12. Das and co-worker's $\mathrm{InCl}_{3}$ catalysed rearrangements under microwave irradiation.

Hoberg reported that reaction with TMSOTf and TMSallyl in $\mathrm{CH}_{2} \mathrm{Cl}_{2}$ using $\mathbf{1 . 1 2}$ as the substrate gave $\mathbf{1 . 1 3}$ in $91 \%$ yield with an $88: 1$ ratio of $\alpha: \beta$ epimers (Scheme 1.8). ${ }^{25}$

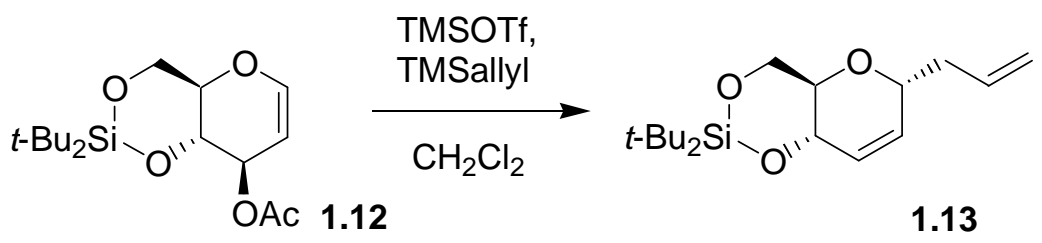

Scheme 1.8. Reaction of di-tert-butylsilyl-glucal with TMSallyl and TMSOTf.

This and similar reactions extend the carbon chain of the base carbohydrate. Thus these reactions are useful to produce chiral synthons for further use in the total synthesis of naturally occurring compounds or their analogues. That the absolute stereochemistry of carbohydrates is known enables their use in asymmetric synthesis without the use of expensive chiral reagents. They are the largest single group in what is known as chiral pool reagents. ${ }^{26}$ 
The earliest total synthesis of a complex natural product was Emil Fischer's synthesis of glucose from D-mannonic acid lactone in 1890 (Scheme 1.9).

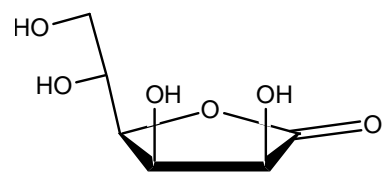

D-mannonic acid lactone

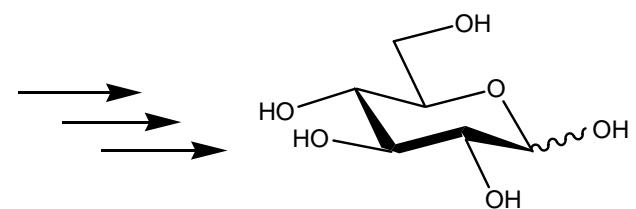

D-glucose

Scheme 1.9. Fischer's total synthesis of glucose.

At the time this was a monumental achievement given the analytical tools of the day. More recently, Nicolaou has used carbohydrates as starting materials for many syntheses of complex natural products. For instance in 1981 Nicolaou's group reported the synthesis of leucomycin $\mathrm{A}_{3} \mathbf{1 . 1 4}$ and carbomycin $\mathrm{B}^{27,28}$ using D-glucose as a starting material, as it possessed three of the required six stereocenters (Scheme 1.10).

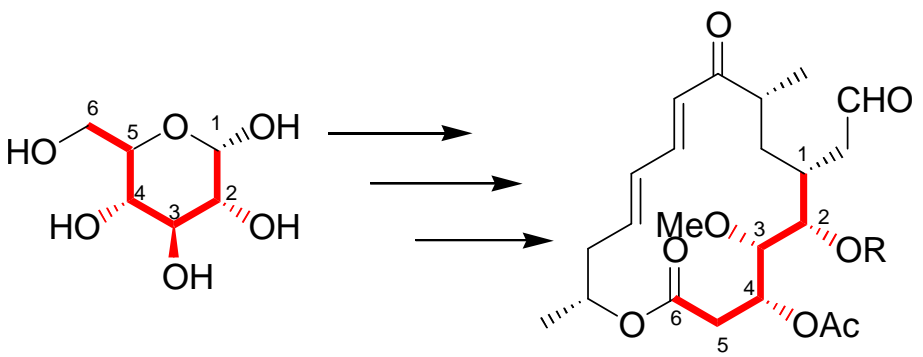

1.14

Scheme 1.10. Leucomycin A3 1.14 showing stereocenters derived from D-glucose starting material.

Nicolaou has also reported the synthesis of amphotericin $\mathrm{B}^{29}$ and swinholide $\mathrm{A}^{30}$ using carbohydrates as base building blocks for the more complex structures. Carbohydrates were also used as the starting point for several of the cyclic ether rings in the brevetoxin series of syntheses reported from the same research group. ${ }^{31-33}$ Other researchers have also made extensive use of carbohydrates in total synthesis. ${ }^{34}$ 


\subsection{Cyclopropanation of carbohydrates}

To further extend the utility of glycals in organic synthesis, much work has been done to functionalise the alkene moiety. One of the ways to add further functionality is via cyclopropanation. There are three main methods for the cyclopropanation of 1,2glycals. These are the Simmons-Smith reaction, reaction with a diazo ester and reaction with a dihalocarbene. The Simmons-Smith reaction uses diiodomethane and a zinc/copper couple to convert alkenes into unsubstituted cyclopropanes. ${ }^{35}$ This occurs through transfer of a methylene group from an organometallic intermediate to the double bond. In the presence of an allylic alcohol or ether substituent this reaction becomes very stereoselective as shown in Scheme 1.11, where the cyclopropanated glucals were generated in high yield as one isomer.
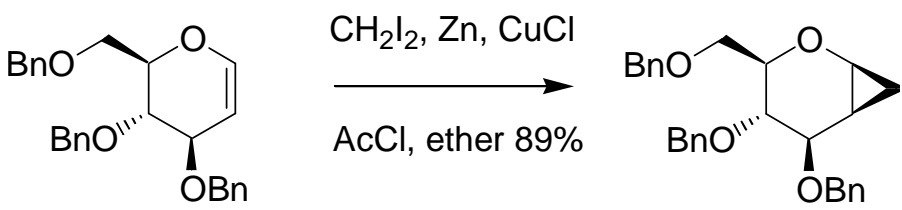<smiles>BrC(Br)(Br)c1ccccc1</smiles>
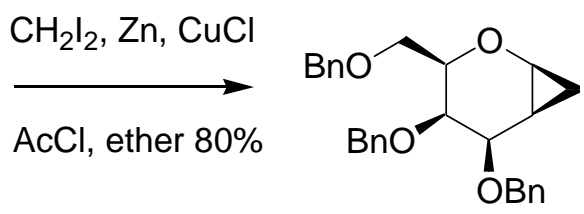<smiles>C[C@H]1OC=C[C@@H](O)[C@H]1OCc1ccccc1</smiles>

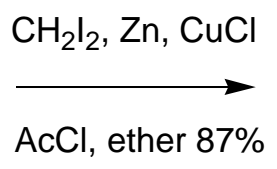<smiles>C[C@H]1OC2CC2[C@H](OCc2ccccc2)[C@H]1OCc1ccccc1</smiles>

Scheme 1.11. Simmons-Smith cyclopropanations.

The cyclopropane adds syn to the allylic alcohol. This is because the zinc co-ordinates to the allylic alcohol in a so-called 'butterfly' intermediate and delivers the methylene to the syn face of the glycal producing cyclopropanes with diastereoselectivities as high as 300:1 (Figure 1.13). ${ }^{36}$ 


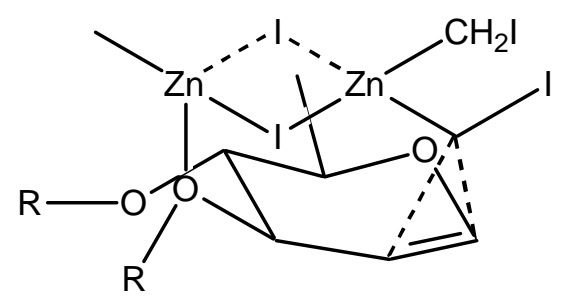

Figure 1.13. Allylic direction of Simmons-Smith cyclopropanation showing the 'butterfly' intermediate.

Nagarajan etal reported an example of this reaction in $1995 .^{37}$ Several benzyl protected glycals were cyclopropanated in high yield with excellent selectivity using acetyl chloride as an activator. ${ }^{38}$ It is postulated that the acetyl chloride reacts with any water present to "super dry" the reaction, and any $\mathrm{HCl}$ produced serves to remove the oxide layer from the zinc surface. In these systems the C-3 substituent controls the facial selectivity of the cyclopropanation and overrides the steric effects from the C-4 and C-6 substituents.

An improved method for the Simmons-Smith is the Furukawa modification using diethylzinc instead of the metallic couple. ${ }^{39}$ This modification is now used extensively, as it is less harsh and more reproducible than the initial method. A wide range of protected glycals have been synthesised using this methodology with excellent yields and stereoselectivities reported (Table 1.1). ${ }^{40}$

The table shows that this reaction is compatible with a range of protecting groups, giving excellent selectivity and yield. However, the authors suggest that yield decreases as steric effects increase (entry five), eventually leading to failure of the cyclopropanation in extreme cases (entry six). This does not account for the high yield shown in entry four. The low yield observed in entry five could be attributed to the 
instability of the protecting group affecting the overall yield rather than steric influences.

Table 1.1. Cyclopropanations of protected glucals using the Furukawa modification of the SimmonsSmith reaction cyclopropanation.

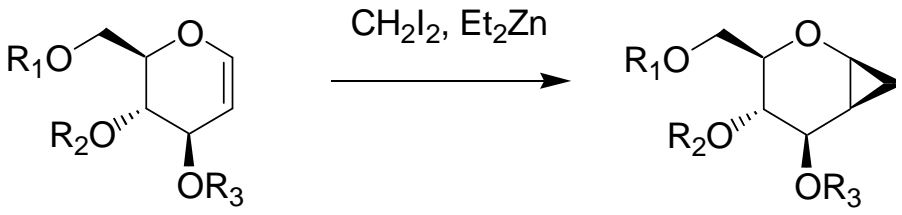

\begin{tabular}{|c|c|c|c|c|c|}
\hline Entry & $\mathbf{R}_{\mathbf{1}}$ & $\mathbf{R}_{\mathbf{2}}$ & $\mathbf{R}_{\mathbf{3}}$ & Yield (\%) & $\boldsymbol{\alpha}: \beta$ \\
\hline 1 & $\mathrm{Bn}$ & $\mathrm{Bn}$ & $\mathrm{Bn}$ & 92 & $\beta$ only \\
\hline 2 & $\mathrm{Me}$ & $\mathrm{Me}$ & $\mathrm{Me}$ & 94 & $\beta$ only \\
\hline 3 & $\mathrm{TBS}$ & $\mathrm{H}$ & $\mathrm{H}$ & 88 & $\beta$ only \\
\hline 4 & \multicolumn{2}{|c|}{$-\mathrm{Si}(t \mathrm{Bu})_{2}$} & $\mathrm{H}$ & 96 & $250: 1^{\mathrm{a}}$ \\
\hline 5 & \multicolumn{2}{|c|}{$-\mathrm{C}(\mathrm{Me})_{2}$} & $\mathrm{H}$ & 33 & $80: 1$ \\
\hline 6 & $\mathrm{TBS}$ & $\mathrm{TBS}$ & $\mathrm{TBS}$ & 0 & - \\
\hline
\end{tabular}

An unexpected result with this methodology was reported by Lorica et al who isolated cyclopropane $\mathbf{1 . 1 5}$ as the major product from their cyclopropanation of glucal $\mathbf{1 . 7}$ (Scheme 1.12). ${ }^{41}$<smiles>CC(=O)OCC1OC=C[C@@H](OC(C)=O)[C@H]1OC(C)=O</smiles>

1.7

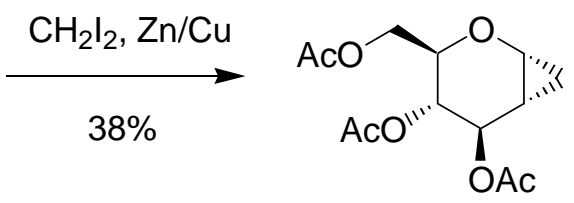

1.15

Scheme 1.12. Loricas synthesis of cyclopropane 1.15.

The authors suggest that the observed stereochemistry is due to the steric hindrance of the upper face by the acetate groups, which implies that careful selection of the protecting groups is required for optimal stereoselectivity. Hoberg etal have since 
reported a method to reverse the stereoselectivity associated with acetate protecting groups. $^{40}$ Cyclopropanation of the diol $\mathbf{1 . 1 6}$ followed by quenching with $\mathrm{Ac}_{2} \mathrm{O}$ generates the diacetyl protected cyclopropane $\mathbf{1 . 1 7}$ in $85 \%$ yield and 8:1 $\beta: \alpha$ selectivity (Scheme 1.13).<smiles>C[C@@H]1OC=C[C@H](O)[C@H]1O</smiles>

1.16

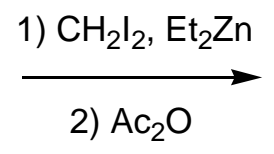

2) $\mathrm{Ac}_{2} \mathrm{O}$

1.17

Scheme 1.13. Hobergs modified reaction scheme.

The Simmons-Smith methodology has also been applied to more complex systems as shown in Figure 1.14. Synthesis of cyclopropane 1.18 (Figure 1.14) occurred stereoselectivity in $93 \%$ yield, while that of $\mathbf{1 . 1 9}$ with no allylic alcohol gave a 1:1 mix of the cyclopropanes. More complex unsaturated carbohydrates have also been cyclopropanated. Danishefsky and co workers synthesised cyclopropane $\mathbf{1 . 1 8}$ as an early stage intermediate towards epothilones $\mathrm{A}$ and $\mathrm{B},{ }^{42}$ while Boeckmann and coworkers synthesised cyclopropane $\mathbf{1 . 1 9}$ during the formation of (-)-calcimycin ( Figure $1.14){ }^{43}$<smiles>C[C@H](COc1ccccc1)[C@H]1OC2C=C[C@H]2[C@@H]1O</smiles>

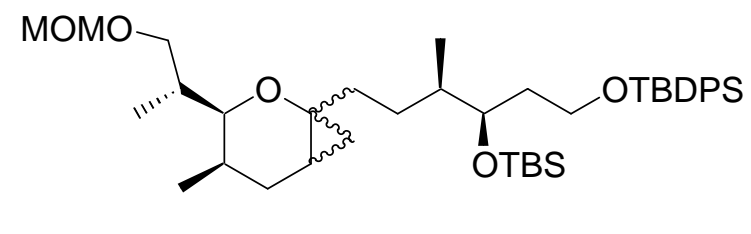

\subsection{9}

Figure 1.14. Complex carbohydrate-derived cyclopropanes synthesised using Simmons-Smith methodology.

The second method is the reaction of glycals with diazo compounds. This approach is not as common as the Simmons-Smith method due to the problems associated with diazo compounds. The reaction occurs by metal-catalysed decomposition of the diazo 
compound to nitrogen and a carbene, which then adds across the alkene forming the cyclopropane. The major advantage of this method is that the reaction is commonly done with $\alpha$-diazo-esters, giving products that are doubly activated donor-acceptor cyclopropanes, with the ester group providing a site for future elaboration. Initial studies of this cyclopropanation gave low yields and poor stereoselectivity. More recent studies have improved both. Henry and Fraser-Reid found that cyclopropanation of tri-O-tert-butyldimethylsilyl-D-glucal with copper powder as the catalyst gave exclusively $\beta$ cyclopropane $\mathbf{1 . 2 0}$ in $92 \%$ yield (Scheme 1.14 ). ${ }^{44}$ However the tri- $O$ benzyl analog gave only $34 \%$ yield with no stereoselectivity.

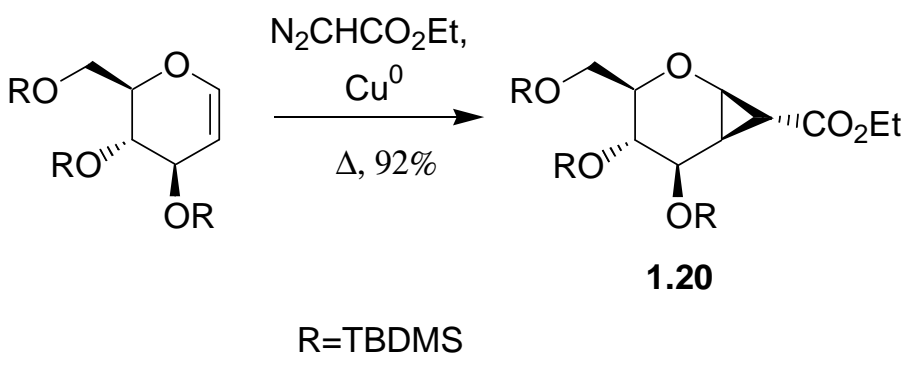

Scheme 1.14. Synthesis of cyclopropane 1.20.

Hoberg and Claffey found that by using a rhodium catalyst, cyclopropanation was achieved in good to excellent yields with a wide variety of protecting groups (Table $1.2)^{45}$

In these examples the $\alpha$ diastereomer was predominantly obtained. It was postulated by the authors that this is due to a steric control process, particularly in the case of larger protecting groups. It is also interesting to contrast the first entry in this table with entry six in Table 1.1 where with identical protecting groups no cyclopropanated product was observed. 
Table 1.2. Results of cyclopropation of glycals with ethyl diazoacetate.

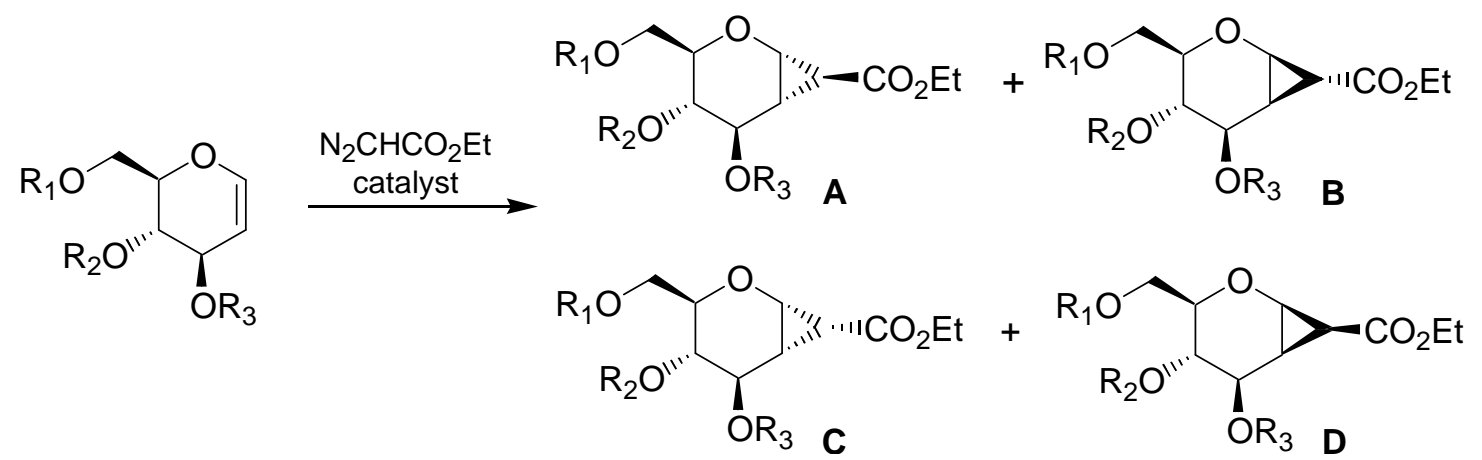

\begin{tabular}{|c|c|c|c|c|c|c|c|c|}
\hline & \multirow{2}{*}{$\mathbf{R}_{\mathbf{1}}$} & \multirow{2}{*}{$\mathbf{R}_{\mathbf{2}}$} & \multirow{2}{*}{$\mathbf{R}_{\mathbf{3}}$} & Catalyst $^{\mathrm{a}}$ & \multirow{2}{*}{ Yield } & \multicolumn{4}{|c|}{ Product Ratio } \\
\cline { 6 - 9 } & & & & & $\mathbf{A}$ & $\mathbf{B}$ & $\mathbf{C}$ & $\mathbf{D}$ \\
\hline TBS & TBS & TBS & $\mathrm{CuO}$ & 92 & 0 & 100 & 0 & 0 \\
\hline TBS & TBS & TBS & $\mathrm{Rh}_{2}(\mathrm{OAc})_{4}$ & 81 & 97 & 3 & 0 & 0 \\
\hline TBS & Ac & Ac & $\mathrm{Rh}_{2}(\mathrm{OAc})_{4}$ & 93 & 94 & 2 & 2 & 3 \\
\hline TIPS & TIPS & TIPS & $\mathrm{Rh}_{2}(\mathrm{OAc})_{4}$ & 66 & 91 & 3 & 3 & 3 \\
\hline Bn & Bn & Bn & $\mathrm{Rh}_{2}(\mathrm{OAc})_{4}$ & 44 & 76 & 8 & 8 & 8 \\
\hline Ac & Ac & Ac & $\mathrm{Rh}_{2}(\mathrm{OAc})_{4}$ & 73 & 81 & 6 & 4 & 9 \\
\hline
\end{tabular}

${ }^{\mathrm{a}}$ All catalysts $1 \mathrm{Mol} \%$ loading

The third method is the addition of a dihalocarbene to the glycal. This reaction is also sterically directed and is carried out most commonly in the appropriate haloform in the presence of a strong base. The resulting dihalocyclopropane can be isolated or reduced by lithium aluminium hydride to the non halogenated cyclopropane. While the reaction has been known for 40 years $^{46}$ it is only recently that Nagarajan has developed a general synthetic sequence that has been applied to several protected glycals (Table 1.3). ${ }^{47}$

The addition is directed by the C-3 substituent whose steric bulk directs the cyclopropane to the opposite face to that derivatised by the Simmons-Smith reaction. 
Thus it is possible to control the stereochemistry of the cyclopropane formed during the reaction by varying the reagents and conditions.

Table 1.3. Results of cyclopropanations using Nagarajan's method.<smiles>[R3]C1C=COC([R])C1[R2]</smiles>

\begin{tabular}{|c|c|c|c|c|c|}
\hline Entry & $\mathbf{R}_{\mathbf{1}}$ & $\mathbf{R}_{\mathbf{2}}$ & $\mathbf{R}_{\mathbf{3}}$ & Yield \% & Cyclopropane \\
\hline 1 gluco- & $\mathrm{MeOCH}_{2}(\beta)$ & $\mathrm{MeO}(\alpha)$ & $\mathrm{MeO}(\beta)$ & 82 & $\alpha$ \\
\hline 2 gluco- & $\mathrm{BnOCH}_{2}(\beta)$ & $\mathrm{BnO}(\alpha)$ & $\mathrm{BnO}(\beta)$ & 84 & $\alpha$ \\
\hline 3 galacto- & $\mathrm{BnOCH}_{2}(\beta)$ & $\mathrm{BnO}(\beta)$ & $\mathrm{BnO}(\beta)$ & 92 & $\alpha$ \\
\hline 4 galacto- & $\mathrm{Me}(\alpha)$ & $\mathrm{BnO}(\beta)$ & $\mathrm{BnO}(\beta)$ & 95 & $\beta$ \\
\hline 5 gluco- & $\mathrm{H}$ & $\mathrm{BnO}(\alpha)$ & $\mathrm{BnO}(\beta)$ & 55 & $\alpha$ \\
\hline
\end{tabular}

\subsection{Reactions of cyclopropanated carbohydrates}

The synthetic exploitation of 1,2-cyclopropanated carbohydrates revolves around the control of the cyclopropane ring opening process and the generation of predictable reaction products. Two main strategies have been used by several research groups. These are the electrophilic ring opening at the C1-C7 position (pathway A) or a Lewis acid assisted pyran ring expansion via a Ferrier-like rearrangement (pathway B), as shown in Scheme 1.15. 


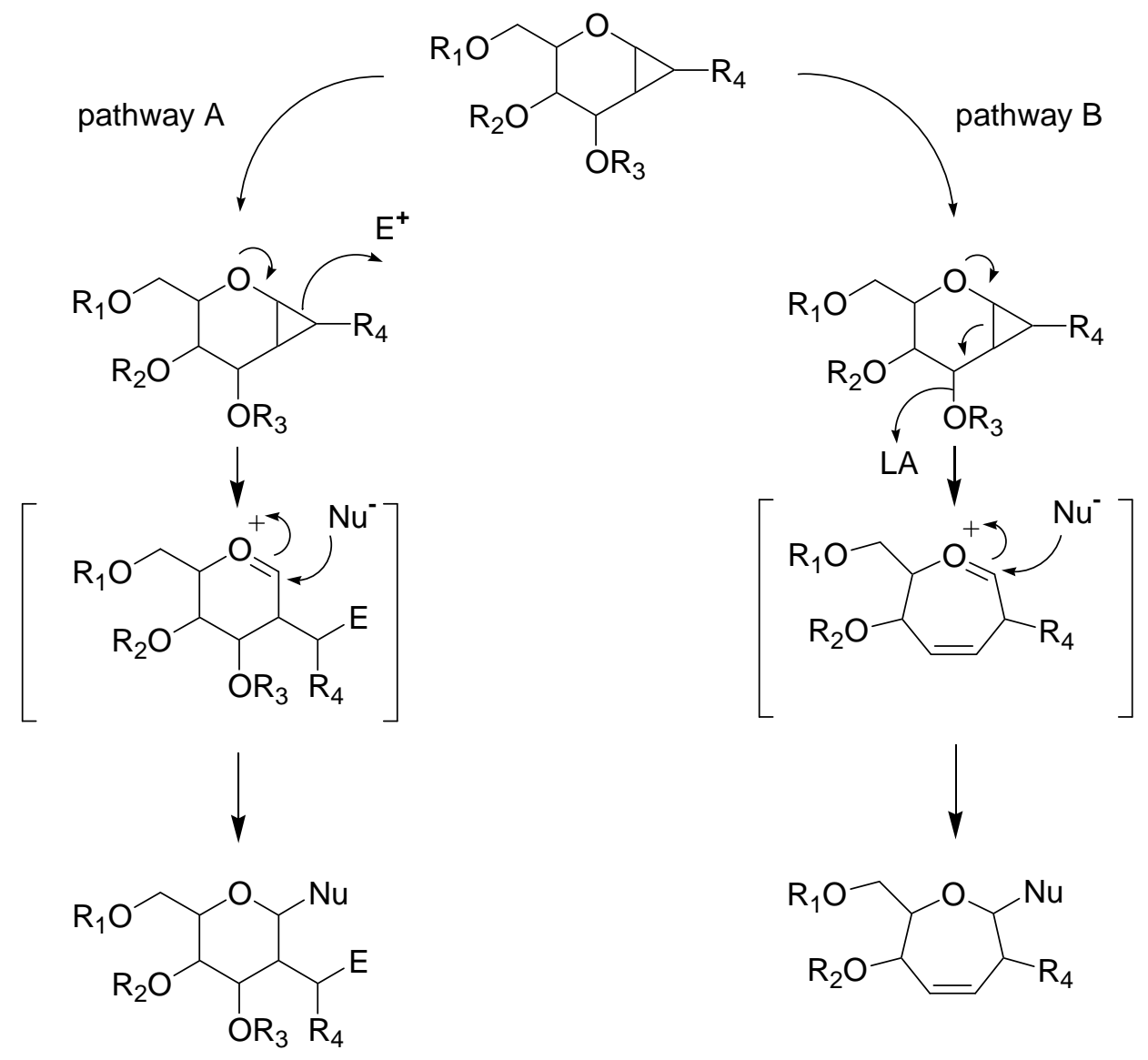

Scheme 1.15. Possible paths for opening of the cyclopropane ring.

Pathway A leads to C-2 substituted glycosides. An electrophile activates the cyclopropane methylene giving an oxonium ion intermediate, which is quenched by a nucleophile. These reactions were initially performed with mercury (II) salt activation of the ring opening. More recently, Danishefsky has used NIS in the cyclopropane opening to access the gem-dimethyl system present in epothilone A and B (Scheme 1.16). ${ }^{42}$ This eliminates the need for use of toxic mercury salts.

Transition metals have also been used to mediate this ring opening. Madsen has demonstrated that reaction of the cyclopropane with the platinum species Zeise's dimer leads to C-2 branched glycosides $\mathbf{1 . 2 1}$ (Scheme 1.17). ${ }^{48}$ This proceeds via an oxidative insertion of platinum (II) into the cyclopropane to generate a metallocyclobutane intermediate $\mathbf{1 . 2 2}$ 

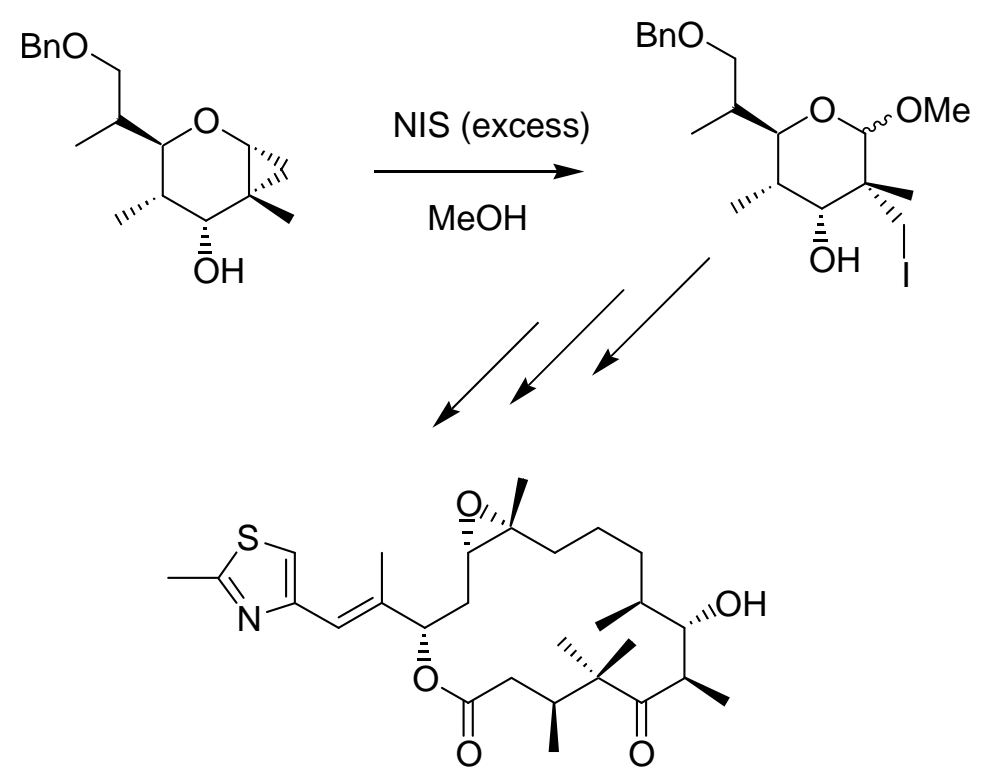

Epothilone B

Scheme 1.16. Installation of a gem-dimethyl moiety on the road to the epothilones.

A restriction on this reaction is the inability to directly form C-1 branched glycosides, as literature examples have only used Bronsted nucleophiles (mostly alcohols) as the glycosyl acceptors.

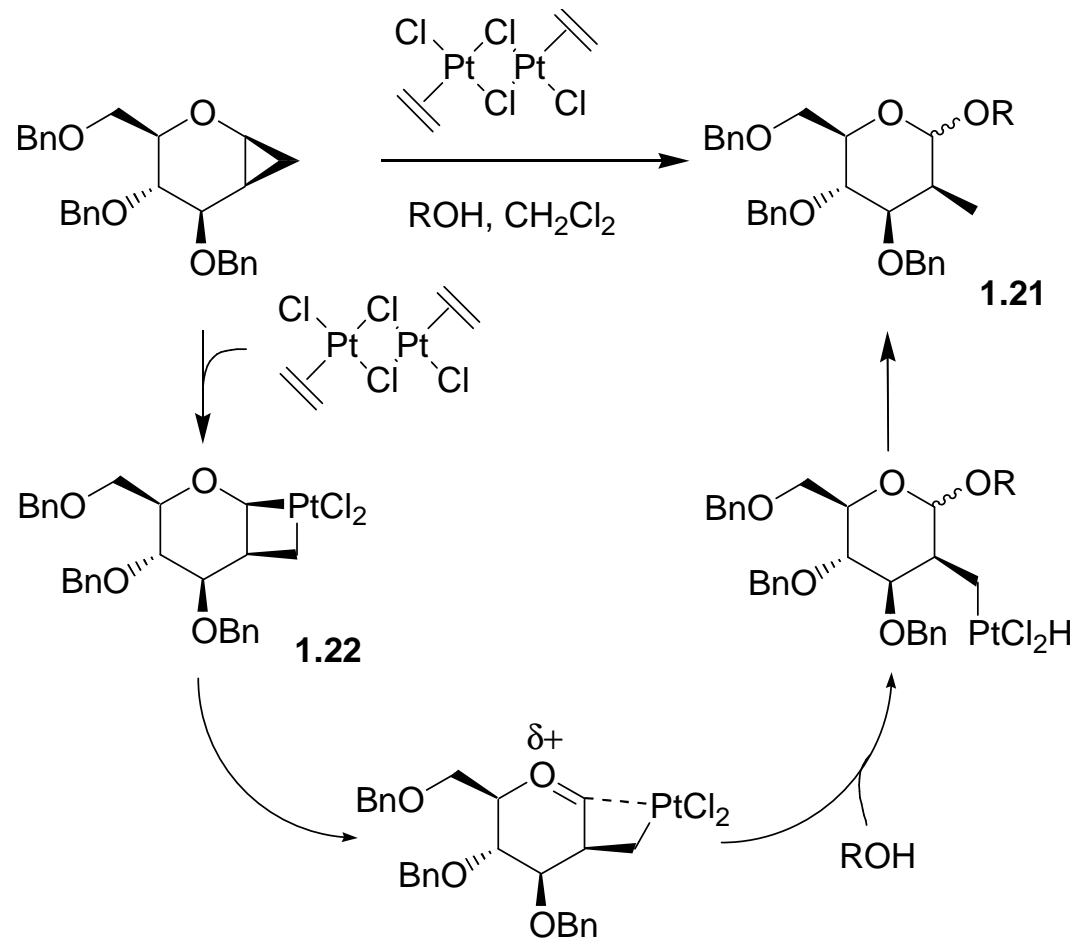

Scheme 1.17. Madsen's platinium catalysed opening of cyclopropanated glucal. 
The second pathway (pathway B from Scheme 1.15) is pyran to oxepine ring expansion, which is also driven by a release of ring strain in the cyclopropane. The Lewis acid activates the leaving group at the $\mathrm{C}-3$ position, and the elimination opens the cyclopropane across the C-1-C-2 bond, generating an oxonium ion. This intermediate can then be converted into any of 3 oxepines $1.23,1.24$, or 1.25 depending on protecting groups and reaction conditions (Scheme 1.18). ${ }^{49}$

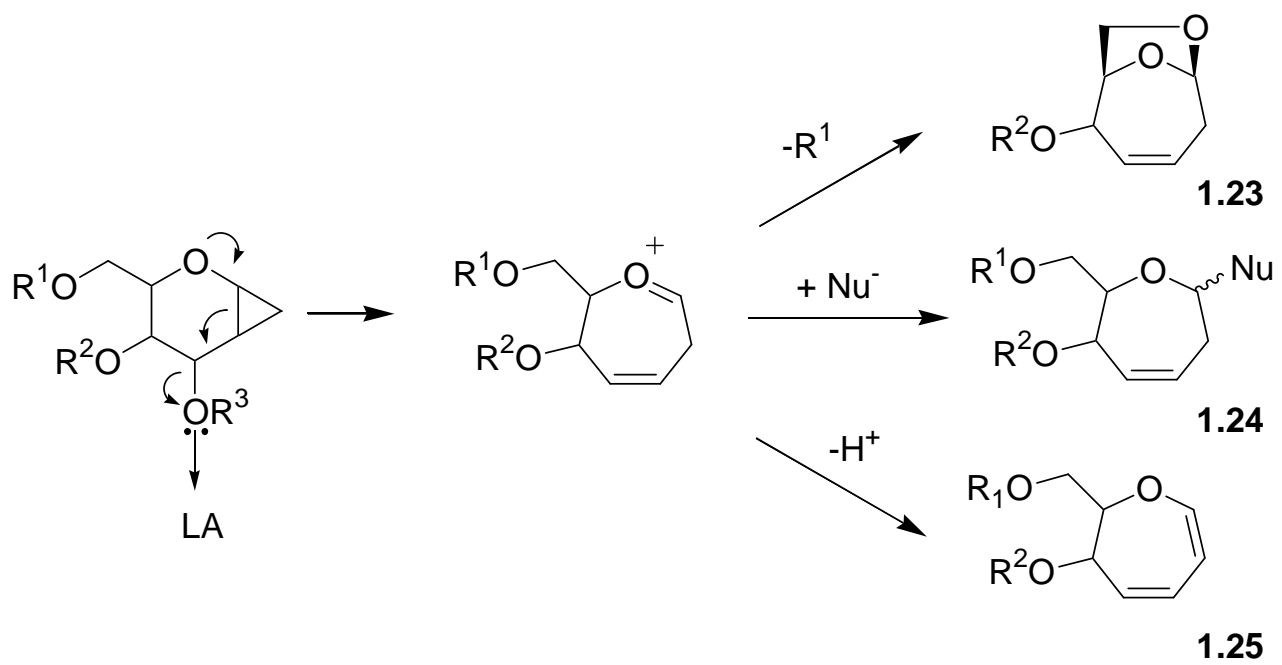

Scheme 1.18. Formation of oxepines from expansion of cyclopropanes.

Sugita and co-workers have investigated ring expansions of structurally similar compounds. ${ }^{50}$ Their cyclopropapyranones $\mathbf{1 . 2 6}$ contain both donor and acceptor groups as part of the ring. Treatment of this system with a Lewis acid leads to the formation of a 1,3-zwitterionic intermediate that could then be intercepted with a nucleophile to yield an oxepanone on workup. The oxonium ion intermediate 1.27 was intercepted with a variety of silyl enol ethers to give the corresponding 4-oxepanones $\mathbf{1 . 2 8}$ (Scheme 1.19). Yields ranged from 24-88 \% and stereoselectivities from 2:1 to 25:1. This depended on the solvent and Lewis acid chosen. The authors believed that the selectivity was due to an anomeric effect from the ring oxygen in the zwitterionic intermediate but did not elaborate further on this hypothesis. 


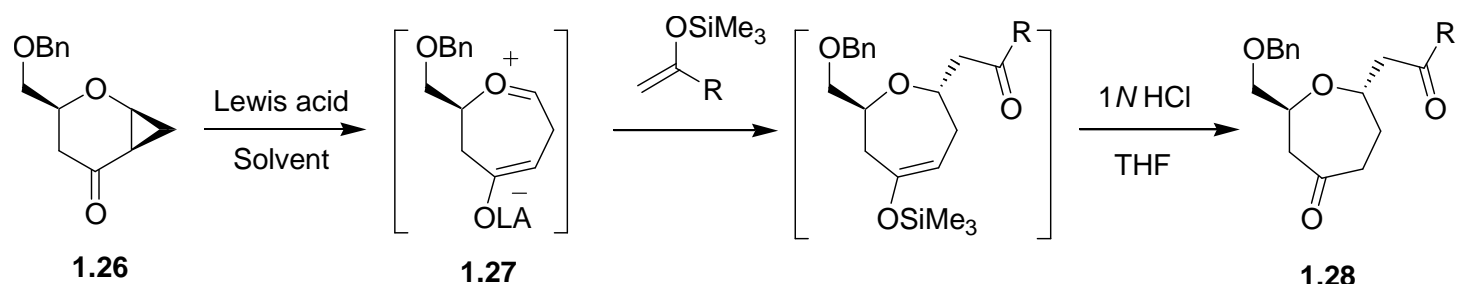

Scheme 1.19. Sugita's expansion of cyclopropapyranones.

\subsection{Oxepine rings in naturally occurring compounds}

A wide range of natural products isolated from both terrestrial and marine sources contain a seven-membered oxacycle as part of their molecular backbone as shown in Figure 1.15.

Sample structures range from the monocyclic rogioloxepane $\mathbf{1 . 2 9}^{51}$ and isolaurepinnacin $\mathbf{1 . 3 0}^{52}$, through the bi-cyclic lobatrienetriol $1.31^{53}$ and fused ring structures of janoxepin $\mathbf{1 . 3 2},{ }^{54}$ oxepinamide $\mathbf{1 . 3 3},{ }^{55}$ and spirocyclic crambescidin acid $\mathbf{1 . 3 4}{ }^{56}$ to the large ladder polyethers brevitoxin 1.35, ${ }^{57}$ gambierol $1.36^{58}$ and ciguatoxin $1.37 .^{59}$ Biochemical studies have revealed a wide range of biological functions for such compounds including ion channel blocking, antiviral and antifungal activities. All these compounds share a common structural feature in that the seven-membered oxacycles all contain at least one carbon-carbon double bond. IUPAC nomenclature describes saturated sevenmembered oxacycles as oxepanes, and the similar completely unsaturated oxacycles as oxepines. Structures with one double bond have been referred to by a variety of names, but Peczuh ${ }^{60}$ has suggested that these compounds also be referred to as oxepines, and this term shall be used in this thesis. 
<smiles>C#C/C=C\C[C@H](Cl)C1CC=CC[C@@H](C(Br)CC)O1</smiles>

(+)-Rogioloxepane<smiles>C#C/C=C/C[C@@H](Cl)[C@@H]1CC=CC[C@H]([C@H](Br)CC)O1</smiles>

(+)-Isolauerpinnacin<smiles>C=C[C@H]1CCC(C2=CC[C@H](C)C(C)(C)OC2)C[C@H]1C(=C)C</smiles>

Lobatrienetriol 1.31<smiles>CC(C)/C=C1\NC(=O)[C@H](CC(C)C)n2c1nc1c(c2=O)C=CC=CO1</smiles>

Janoxepin<smiles></smiles>

Oxepinamide C $\mathbf{1 . 3 3}$

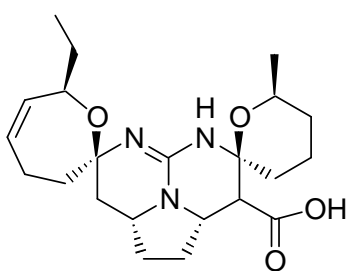

Crambescidin acid $\mathbf{1 . 3 4}$

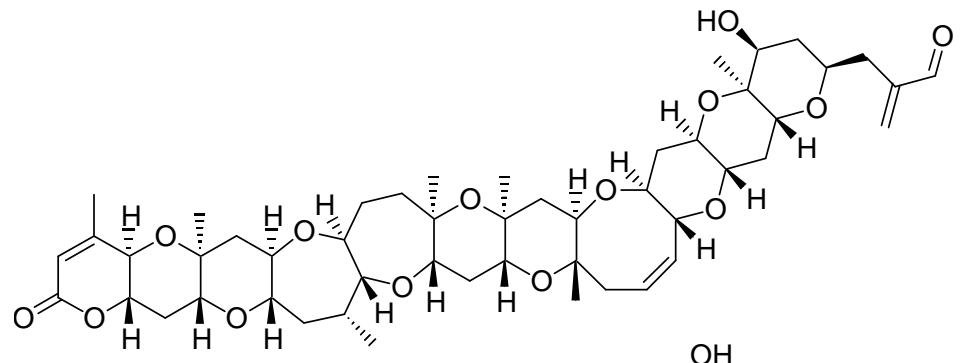

Brevitoxin B $\mathbf{1 . 3 5}$

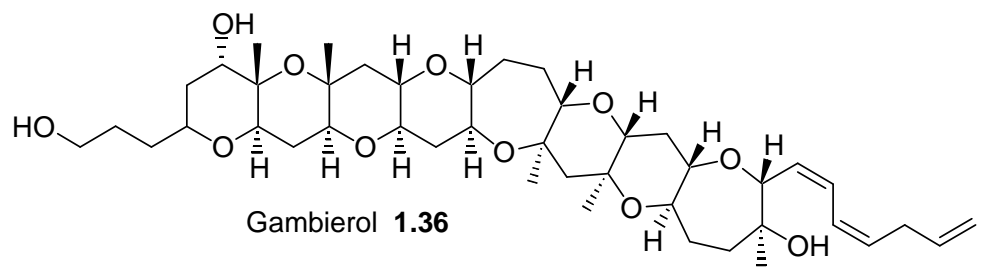

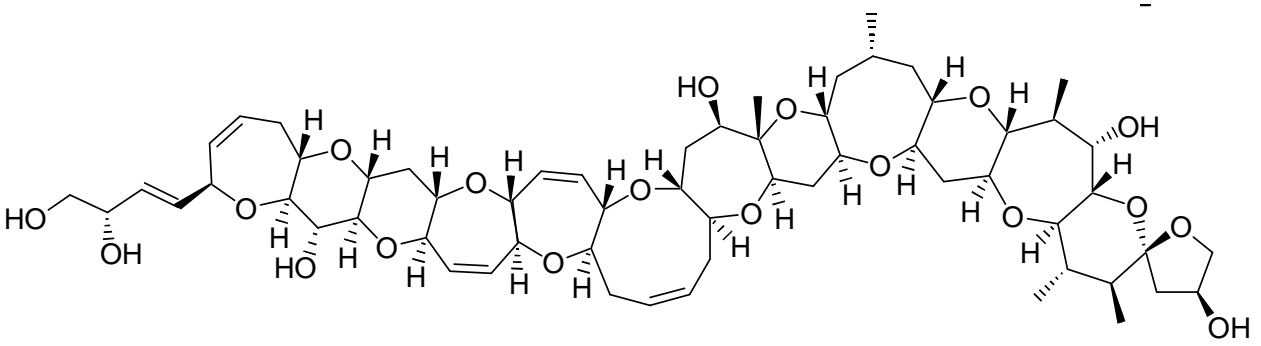

Ciguatoxin 1.37

Figure 1.15. Examples of naturally occurring compounds containing seven-membered oxacycles.

Most of the research on these oxacycles has been driven by the development of methods leading to the synthesis of natural products, mainly in the formation of the polycyclic ethers 1.35-1.37. 


\subsection{Methods of formation of seven-membered rings}

There are two main approaches taken to the synthesis of seven-membered oxacycles: cyclisation via formation of a C-O bond or formation of a C-C bond. ${ }^{60}$

\subsubsection{Seven-membered oxacycle formation via C-O bond formation}

This type of approach takes advantage of the inherent nucleophilicity of oxygen in attacking an electrophilic carbon species.

Lewis acids have been used to construct oxacycles by co-ordinating to an epoxide species. This is a particularly attractive strategy as the epoxide can be formed enantioselectively and cyclisation gives rise to a chiral hydroxyl group.

This is demonstrated with the following examples as shown in Scheme 1.20 as part of total syntheses of the natural products isolaurepinnacin $\mathrm{A}^{61}$ and rogioloxepane $\mathrm{A}^{62}$ which have very similar core structures and only differ in the disposition of the substituents across the oxepine ring oxygen (syn vs anti).

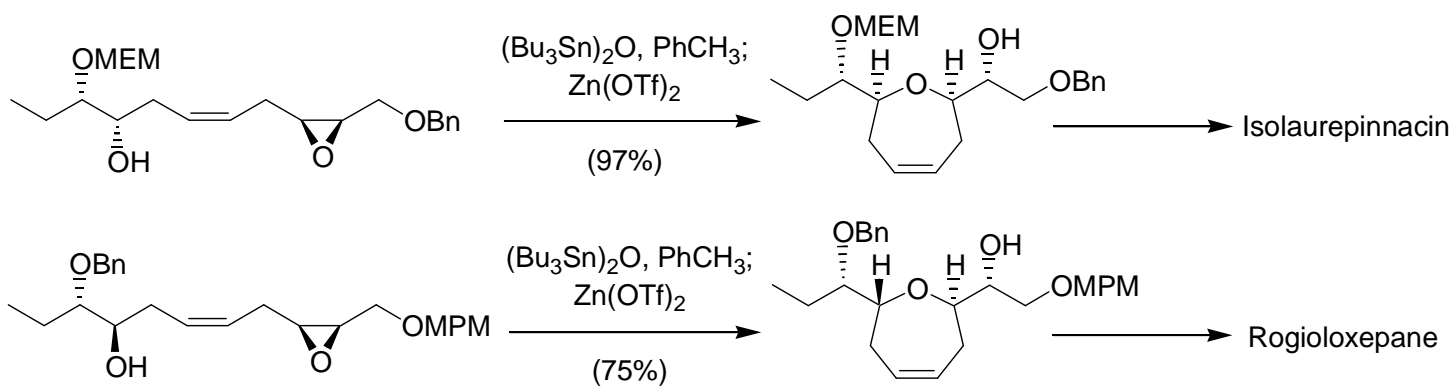

Scheme 1.20. Suzuki's syntheses of isolaurepinnacin A and rogioloxepane A. 
In this scheme the epoxy-alcohols are treated with $\left(\mathrm{Bu}_{3} \mathrm{Sn}\right)_{2} \mathrm{O}$ which forms an alkyltin ether that increases the nucleophilicity of the hydroxyl oxygen, followed by addition of zinc triflate as a Lewis acid to activate the cyclisation step. While the yields are high in these transformations, attempts to apply this methodology to more complex systems were not as successful and the authors put this down to inefficient formation of the alkyltin ether intermediates.

\subsubsection{Seven-membered oxacycle formation via $\mathrm{C}-\mathrm{C}$ bond formation}

Nicolaou was among the first to employ this method in the synthesis of polyethers as part of the synthetic effort towards the total synthesis of large polycyclic ladder compounds. $^{63}$ An olefinic ester 1.38 was methylenated using Tebbe's reagent ${ }^{64}$ to produce the corresponding vinyl ether which was then further transformed with an additional equivalent of Tebbe's reagent to provide the corresponding oxepine $\mathbf{1 . 3 9}$ in moderate yield (Scheme 1.21). A variety of six and seven membered cyclic enol ethers were constructed using this methodology (more details of Tebbe's reagent will be provided in Section 2.5).<smiles>C/C=C\CC[C@]1(C)O[C@H]2C[C@H](O)[C@@H](COc3ccccc3)O[C@H]2C[C@H]1OC(C)=O</smiles>

1.38
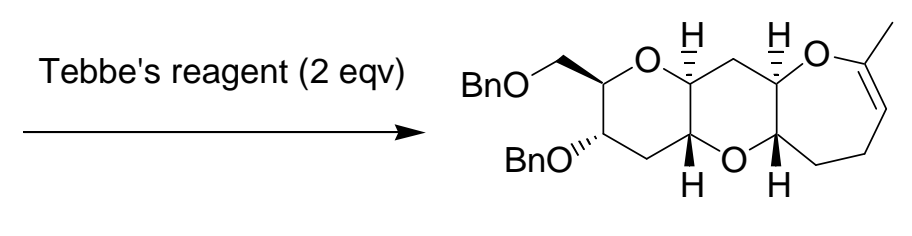

1.39

Scheme 1.21. Oxepine formation involving Tebbe's metathasis.

While useful, the Tebbe's reaction suffers from several problems. The generation of the initial reactive species generates stoichiometric quantities of methane, which may preclude its use on a large scale. The reaction itself requires excess amounts of the reagent to complete the methylenation. Finally, it is difficult to remove the titanocene 
byproduct from the reaction product. This tends to preclude it from any form of largescale application.

A general method for the preparation of cyclic ethers has been applied to the synthesis of oxepane rings as part of the total synthesis of the large ladder polycyclic ethers of the ciguatoxin series. Yamamoto and co-workers applied this method, which involves an intramolecular attack of an allylstannane on an aldehyde, to the synthesis of hemibrevetoxin B. ${ }^{65}$ In Scheme 1.221 .40 was converted to $\mathbf{1 . 4 1}$ in $94 \%$ yield and produced only one diastereoisomer.

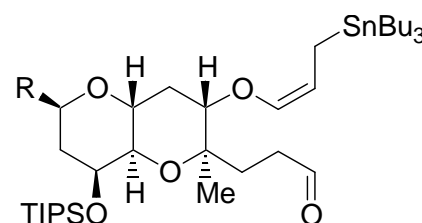

1.40

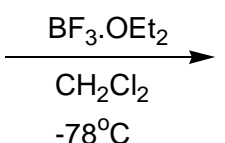

$-78^{\circ} \mathrm{C}$

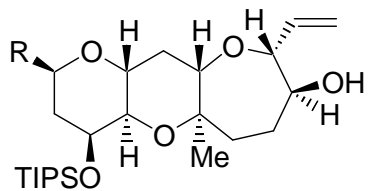

1.41

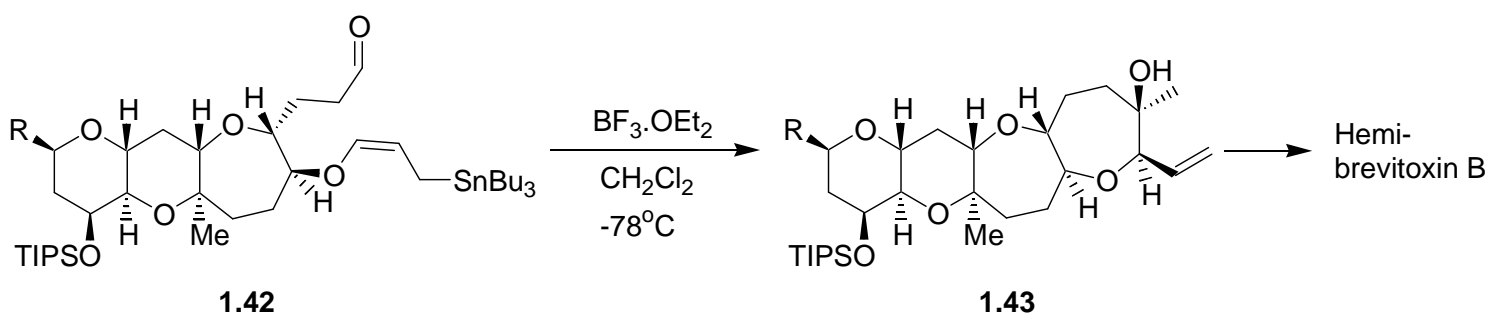

Scheme 1.22. Formation of oxepane rings leading to synthesis of hemibrevetoxin B.

Subsequent formation of another allylstannane and an aldehyde group in several steps is followed by conversion of $\mathbf{1 . 4 2}$ to $\mathbf{1 . 4 3}$ in $98 \%$ yield, again with only one diastereoisomer formed. Other researchers have used variations of this method in the synthesis of similar molecules. However, the presence of tin, which is extremely environmentally toxic, in these reactions also precludes their use in a scaled up synthesis. 
Carbon-carbon bond formation to generate oxepines has come to be dominated by ring closing methasis (RCM), due to the ongoing development of organometallic catalysts for this type of reaction. These advances have largely occurred since the start of this project. RCM has many advantages in the formation of medium-sized rings in general and oxepines in particular. The disconnections are particularly simple, have no stereochemical complications as a result of the ring formation, and the alkene can be formed at any position on the ring as a precursor to further elaboration. The catalysts are compatible with a wide variety of complex functionalities, so can be used as a late stage synthetic step in a total synthesis strategy.

Grubbs' catalysts have been applied to the formation of medium sized cyclic enol ethers (including oxepines) as part of a sequence to construct large polycyclic ethers of type 1.34-1.36. Rainer and co-workers developed a strategy outlined in Scheme 1.23. ${ }^{66}$

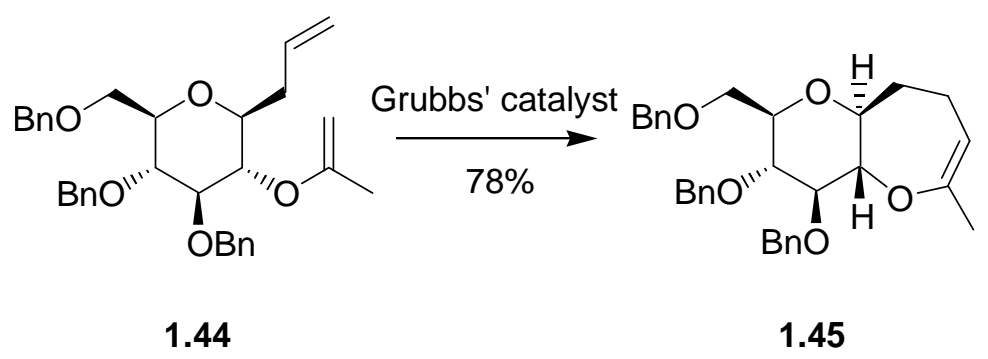

Scheme 1.23. Formation of oxepine ring with Grubbs' catalyst.

Epoxidation of tri- $O$-benzyl glucal followed by addition of allyl magnesium chloride generates a mixture of C-glycosides. Acetylation of the free hydroxyl group followed by methylenation formed the metathesis precursor 1.44. Ring closing metathesis using Grubbs' second generation catalyst gave $\mathbf{1 . 4 5}$ in a $\mathbf{7 8 \%}$ yield and the bicyclic oxepine was now set up for subsequent iterations of epoxidation, nucleophilic attack and cyclisation to continue the growth of the fused polycyclic ether. 
Similarly, Jenkins and Ghost used a chiron starting material to synthesize polycyclic ethers (Scheme 1.24). ${ }^{67} \mathbf{1 . 4 6}$ was treated with $\mathrm{NaH}$ and allyl bromide to give 1.47. Treatment with Grubbs' second generation catalyst gave $\mathbf{1 . 4 8}$ in $87 \%$ yieldwith the oxepine positioned for further elaboration to give access to more complex structures.

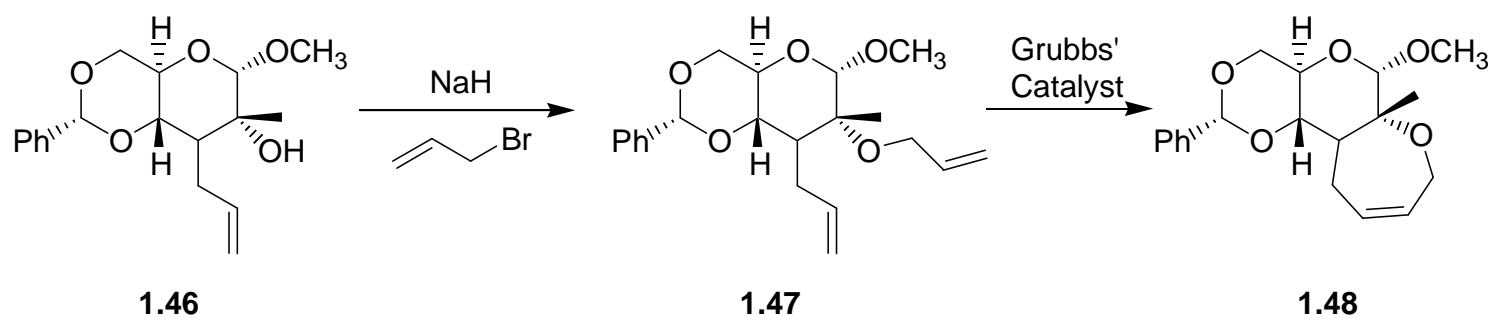

Scheme 1.24. Jenkins and Ghost's oxepine formation.

Clark and co-workers have also used Grubbs' second generation catalyst to form oxepines using an ene-yne system in a synthetic effort towards gamberic acid and gambierol 1.36 (Scheme 1.25). ${ }^{68}$ The authors also note that the success of the ene-yne metathesis depends on the size of the terminal group on the alkyne. Smaller groups $(\mathrm{H}$ or Me) give good yields $(70 \%)$ while the larger TMS group returned only starting materials.

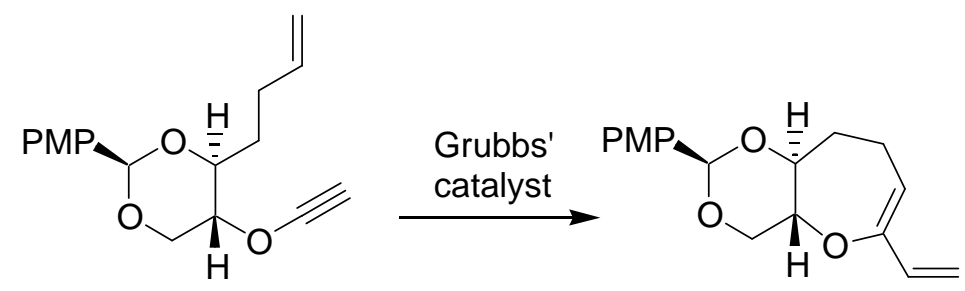

Scheme 1.25. Formation of oxepine ring with Grubbs' catalyst.

Another common method for oxepine formation that has been applied to the synthesis of several natural products is intramolecular attack on an acetal. Overman and coworkers utilised a Prins cyclisation of mixed acetals as part of a synthetic route to (+)isolaurepinnacin (Scheme 1.26). ${ }^{69}$ 


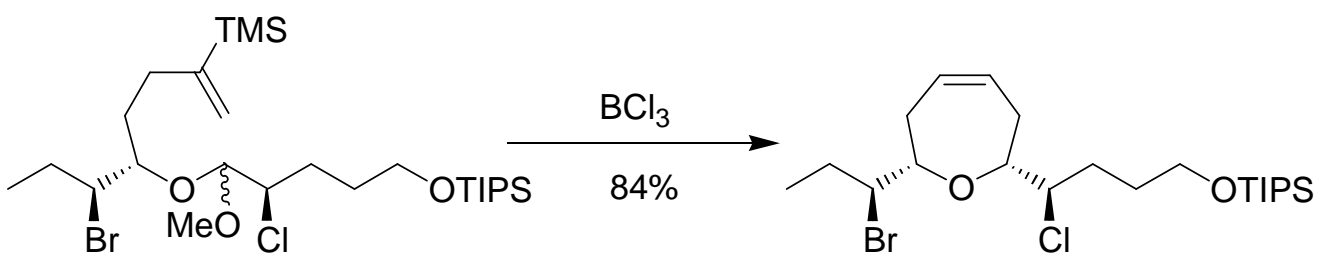

1.49

Scheme 1.26. Formation of oxepine ring by Prins cyclisation.

Treatment of 1.49 with $\mathrm{BCl}_{3}$ selectively cleaves the methoxy acetal to give an $\alpha$-chloro enolether. Upon warming to room temperature an oxonium intermediate is formed which then undergoes cyclisation and olefin formation to give oxepine $\mathbf{1 . 5 0}$ in a $90 \%$ yield. Only one isomer is generated in this reaction.

Nicolaou and co-workers have developed a photochemical coupling of thiocarbonyl compounds to prepare oxepine intermediates as part of the effort to synthesize brevitoxin B (Scheme 1.27). ${ }^{31}$<smiles>CCCCC(=O)C[C@H]1CCCO[C@H]1CC(=O)OC</smiles>

1.51<smiles>CCCC1=C(OC)[C@H](C)C[C@H]2OCCC[C@H]2O1</smiles>

1.55
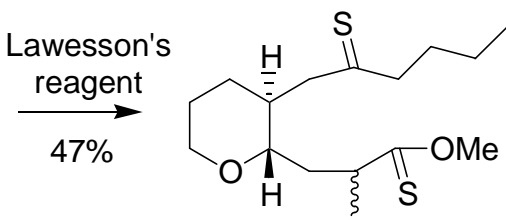

1.52

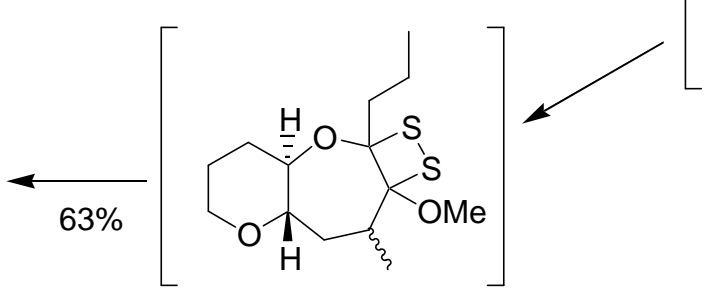

1.54

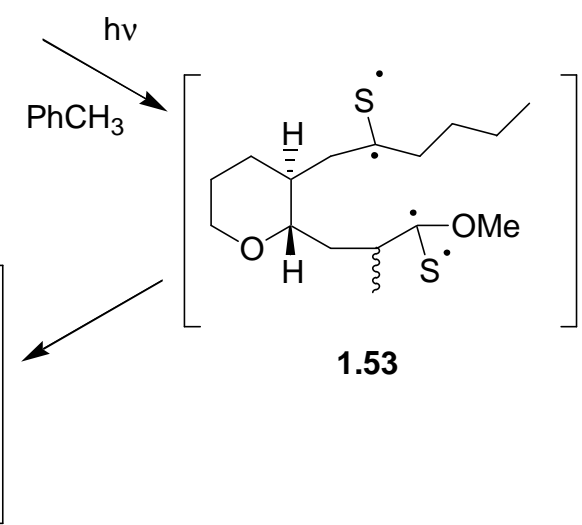

Scheme 1.27. Nicolaou's photochemical generation of oxepine rings.

The ester-keto starting material $\mathbf{1 . 5 1}$ was thionated using Lawesson's reagent to generate the dithiono system $\mathbf{1 . 5 2}$ which upon exposure to UV light generates a bisdiradical species $\mathbf{1 . 5 3}$ which couples to form a 1,2-dithietane 1.54. $S_{2}$ is then expelled 
to give the oxepine $\mathbf{1 . 5 5}$ in $63 \%$ yield. This strategy could also possibly be used in a late stage connection of two complex fragments in the formation of a natural product. Hirama and co-workers have also used a photochemical rearrangement as part of an investigation into the synthesis of parts of the ciguatoxin structure (Scheme 1.28). Photoelectrocyclic reaction of the diene $\mathbf{1 . 5 6}$ forms cyclobutene $\mathbf{1 . 5 7}$ which upon ozonolysis followed by reduction by $\mathrm{PPh}_{3}$ gives oxepanedione $\mathbf{1 . 5 8}$ which can be furthur transformed into oxepine 1.59. This methodology can also be used to construct eight- and nine-membered rings.

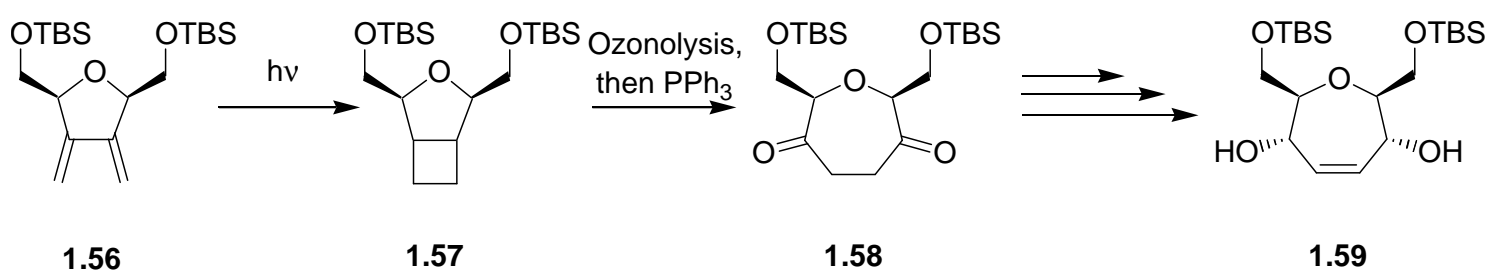

Scheme 1.28. Hirama's photochemical generation of oxepine rings.

\subsection{Ring expansion of cyclopropanated carbohydrates}

Hoberg and co-workers have reported the utilisation of the Ferrier rearrangement in combination with a cyclopropanated carbohydrate to generate oxepines in high yield. ${ }^{70}$ Initial work lead to by-products with oxepines isolated as a [4.2.1] bicyclic system 1.60. With labile protecting groups (Ac, Bn, or smaller silyl derivatives) the C-6 alcohol was deprotected under the reaction conditions and competed with the external TMS nucleophile (Scheme 1.29). In cases where the nucleophile had low reactivity $\mathbf{1 . 6 0}$ was formed in up to $78 \%$ yield. With a more robust silyl protecting group at $\mathrm{C}-6$ eg $\left(\mathrm{R}^{1}=\mathrm{R}^{2}\right.$ $\left.=t \mathrm{Bu}_{2} \mathrm{Si}, \mathbf{1 . 6 1}\right)$ oxepines of type $\mathbf{1 . 6 2}$ were formed with a wide variety of nucleophiles. While yields were quite high there was only moderate facial selectivity (at best 1:2 $\alpha: \beta)$. In all reactions elimination to the diene $\mathbf{1 . 6 2}$ competed with nucleophilic attack. 

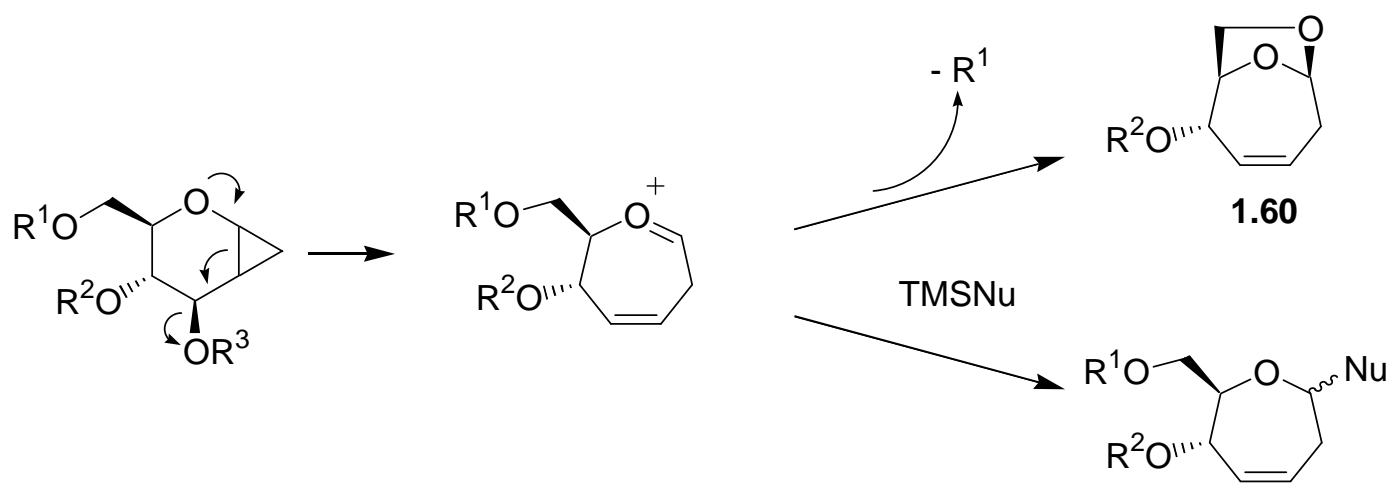

1.62

Scheme 1.29. Formation of [4.1.0] bicyclic system.

The results are summarised in Table 1.4.

Of interest in this series of expansions was the low stereoselectivity in attachment of the anomeric substituent. In fact the predominant $\beta$ stereoselectivity is the opposite to that normally observed in the Ferrier rearrangements. This suggested there were differences in the application of this particular reaction to the different sized ring systems, and that there needed to be more research done on the application of this rearrangement to the formation of oxepine systems. 
Table 1.4. Cyclopropane expansions using TMSOTf.

\begin{tabular}{|c|c|c|c|}
\hline nucleophile & Product & Yield (\%) & Diastereoselectivity $\alpha: \beta$ \\
\hline $\mathrm{TMSN}_{3}$ & & 93 & $1: 2$ \\
\hline TMSallyl & & 92 & $1: 1$ \\
\hline TMSSPh & & 93 & $1: 2$ \\
\hline TMSCN & & 82 & $1: 2$ \\
\hline TESH & & 73 & - \\
\hline TMSpropargyl & & 67 & $1: 1$ \\
\hline OTMS & & 85 & $1: 2$ \\
\hline
\end{tabular}




\subsection{Aims and objectives}

The aim of this project was to investigate further the Ferrier-type rearrangement of cyclopropanated carbohydrates with an eventual view towards making a synthetically useful route to chiral oxepines.

Objectives were:

1) To optimise the synthesis of various protected glycals and the subsequent cyclopropanation of the glycal;

2) Explore the Ferrier-type ring expansion while varying parts of the molecule and reaction conditions. The main areas of interest were:

a) The examination of different protecting groups at the C-4 and C-6 positions;

b) The variation of the base sugar structure;

c) The examination of a wider variety of nucleophiles;

d) The investigation of different leaving groups at the C-3 position;

These areas were to be examined with the underlying intention of increasing the stereoselectivity of the rearrangement;

3) Gain an increased understanding of the underlying reaction mechanisms involved in the Ferrier rearrangement as applied to the cyclopropanated glycals. This would be achieved by:

a) Exhaustive NMR analysis of reaction by-products;

b) Utilisation (where possible) of isotopic labelling studies;

4) Further functionalisation of the oxepine products providing a range of synthons for use in a bioactive analogue synthesis series;

5) Extend the methodology to the synthesis of a naturally occurring compound. 


\subsection{A note about NMR assignments in this thesis.}

The oxepins synthesised in the course of this thesis are of two types: mono- and bicyclic ring systems. As such they have different IUPAC numbering conventions. The numbering also can alter depending on the substituents. However, during the course of this thesis the ring systems will be compared to each other. Therefore, to simplify comparison of data and structures, all NMR assignments have used the numbering scheme shown in Figure 1.16.
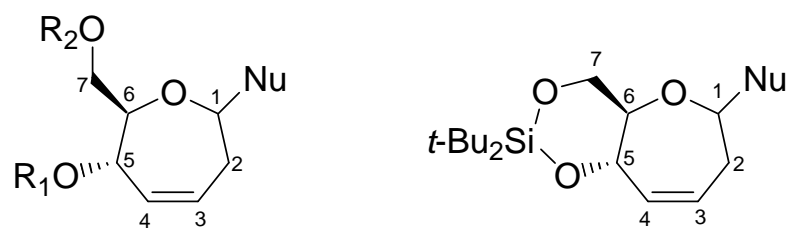

Figure 1.16. Numbering conventions used in this thesis for the purposes of NMR assignments.

This numbering scheme highlights the similarities between the oxepine rings, with the carbon that would be considered the 'anomeric' center in the starting carbohydrates numbered $\mathrm{C}-1$ and then subsequent assignments around the ring as usual in carbohydrate structures. This numbering system will also be used in the discussion of positions on the ring in the text.

The IUPAC numbering convention will be applied to the names that appear in the experimental section of the thesis. The author hopes that this will not cause confusion to the reader. 


\section{Chapter 2: Initial studies on the Ring Expansion of Cyclopropanated}

\section{Glycals}

In order to build on Hoberg's successful methodology ${ }^{70}$ discussed in Section 1.7 (Scheme 2.1), it was decided to make some alterations to the previous initial substrate in the choice of main protecting group in an attempt to increase the stereoselectivity of the reaction.

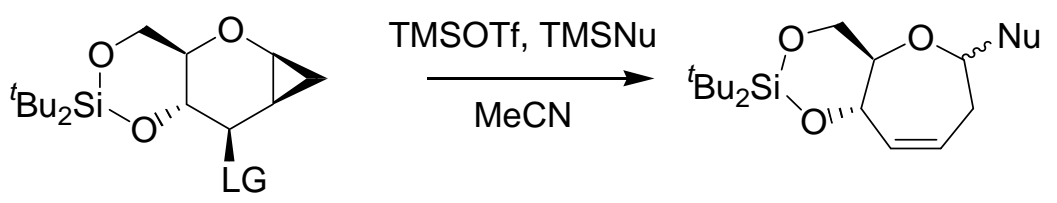

Scheme 2.1. Hoberg's ring expansion methodology.

The di-tert-butylsilyl protecting group, while stable to the reaction conditions, leaves a 1,3-diol moiety following deprotection. To improve the general applicability, of this methodology it would be preferable to selectively reveal these groups independently.

Therefore, the initial plan was to use a stable bulky protecting group at the C-6 position to determine whether this would alter the outcome of the ring expansion reaction. Das and co-workers have demonstrated the effect a group at this C-6 position has on the Ferrier rearrangement. ${ }^{24}$ They showed that by making one face of the molecule less favourable to the approach of a nucleophile leads to an increase in the stereoselectivity of the ring expansion. Previous work with a range of non-silyl protecting groups (acetate, dimethylacetonide and benzyl) had shown that during the ring expansion the protecting group was cleaved from the primary alcohol by the presence of a Lewis acid. $^{40}$ This then acted as an intramolecular nucleophile that competed with the intermolecular nucleophile during the ring expansion (Scheme 2.2). In some cases, 
where the external nucleophile was particularly unreactive, the bicyclic oxepine was the major product isolated.

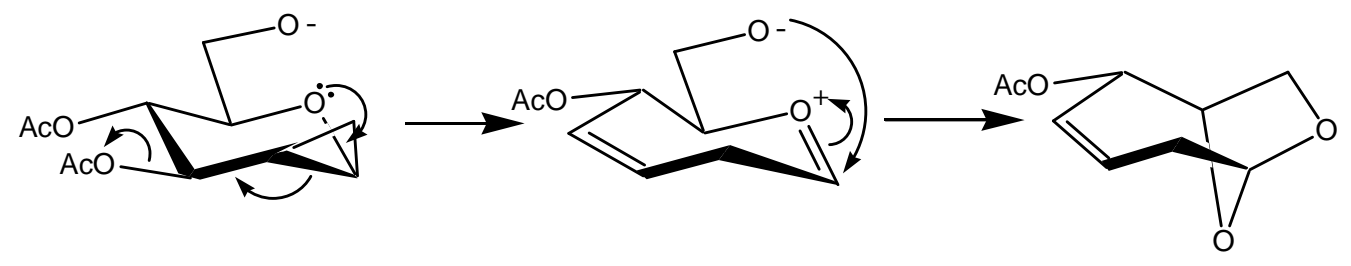

Scheme 2.2. Ring expansion with interception of oxonium intermediate by internal nucleophile.

The triisopropylsilyl ether protecting group was chosen for its expected stability under the reaction conditions and its steric bulk. ${ }^{71}$ It was also decided to change the ring system from glucal to galactal as Danishefsky's work had shown that using tri- $O$-acetylgalactal instead of tri- $O$-acetyl-glucal in the Ferrier rearrangement resulted in a two-fold increase in the stereoselectivity. ${ }^{22}$

\subsection{Investigations of 6-O-TIPS protected cyclopropanated galactal}

Synthesis of the desired cyclopropanated galactal proceeded smoothly in good yields in three steps from D-galactal (Scheme 2.3).

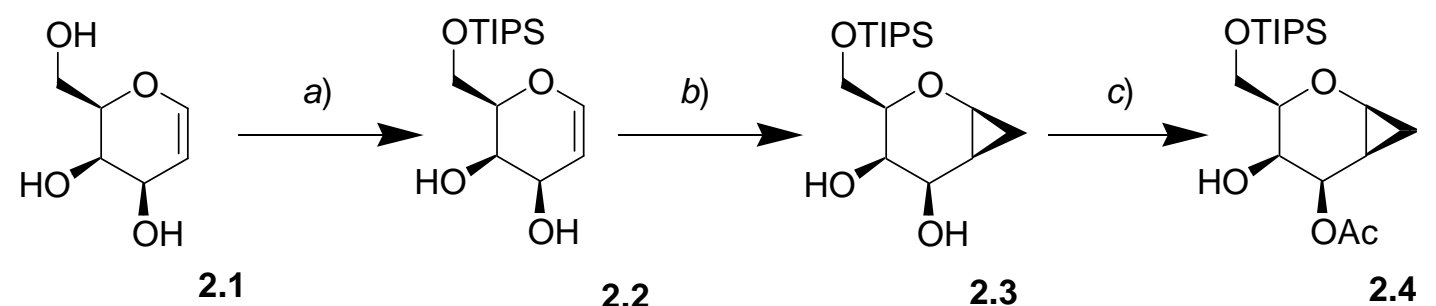

a) TIPS-Cl, TEA, DMF, rt, 66\%. b) $\mathrm{CH}_{2} \mathrm{I}_{2}, \mathrm{Et}_{2} \mathrm{Zn}, \mathrm{Et}_{2} \mathrm{O}$, reflux, $72 \%$.

c) $\mathrm{Ac}_{2} \mathrm{O}, \mathrm{py}, \mathrm{CH}_{2} \mathrm{Cl}_{2}$, DMAP, $99 \%$

Scheme 2.3. Formation of cyclopropane 2.4. 
To this end, galactal $\mathbf{2 . 1}$ was silylated giving silyl ether 2.2 in $66 \%$ yield. Compound 2.2 was then subjected to cyclopropanation by the Furukawa modification of the Simmons-Smith cyclopropanation ${ }^{39}$ to give diol $\mathbf{2 . 3}$ in $72 \%$ yield. Acetylation of both hydroxyl groups of $\mathbf{2 . 3}$ proved to be problematic. It was relatively easy to acetylate the C-3 hydroxyl, but the C-4 hydroxyl proved resistant to acetylation. This is potentially due to its location adjacent to the TIPS group, with steric hindrance the most likely reason for the lack of success of this reaction.

With the cyclopropane $\mathbf{2 . 4}$ in hand, some initial reactions were performed to probe possible conditions conducive to the ring expansion.

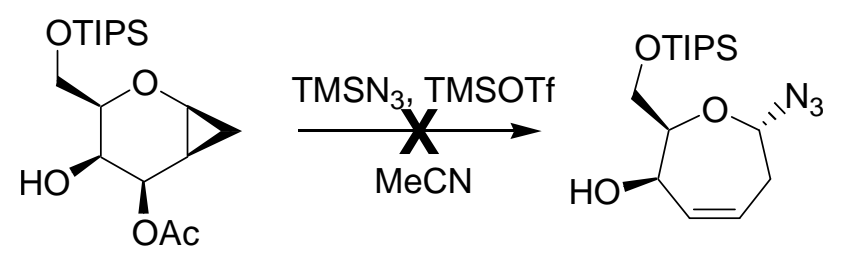

Scheme 2.4. Initial attempts towards a general method for ring expansion.

In an initial attempt (Scheme 2.4), cyclopropane 2.4 was treated with $\mathrm{TMSN}_{3}$, using TMSOTf as the Lewis acid catalyst in $\mathrm{MeCN}$ at $-40{ }^{\circ} \mathrm{C}$. The temperature was subsequently raised to $-20{ }^{\circ} \mathrm{C}$ followed by stirring for six hours, however this gave no reaction by TLC. Repeating the reaction at $0{ }^{\circ} \mathrm{C}$ and stirring for eight hours while warming to ambient temperature resulted in deprotection of the C-6 hydroxyl through removal of the TIPS protecting group. A final attempt at heating the mixture to reflux for two hours resulted in decomposition of the starting material to unknown low $R_{f}$ products that could not be isolated.

Experiences at later stages of the investigation have revealed that having an unprotected alcohol in the cyclopropane molecule provides a proton source. This decomposes the 
catalytic TMS triflate to triflic acid, which then catalyses the deprotection of the silyl protecting group leading to the generation of inseparable reaction products.

\subsection{Investigation of alternative leaving groups}

From this work it was then postulated that a better leaving group than acetate was required to facilitate the initiation of the desired reaction at a temperature low enough to ensure the survival of the attached TIPS group. The leaving groups chosen for this investigation were carbonate, triflate and mesylate.

\subsubsection{Carbonate as the leaving group}

Treatment of diol $\mathbf{2 . 3}$ with carbonyldiimidazole in dry THF for two hours gave carbonate $\mathbf{2 . 5}$ in $54 \%$ yield (Scheme 2.5 ).

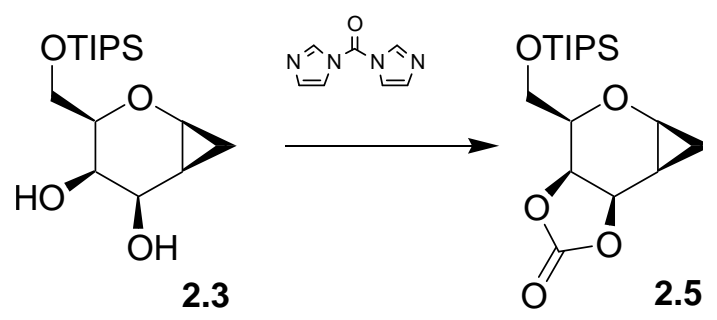

Scheme 2.5. Formation of cyclopropanated galactal 2.5.

Treatment of this substrate with $\mathrm{TMSN}_{3}$ and TMSOTf in $\mathrm{MeCN}$ at $0{ }^{\circ} \mathrm{C}$ only returned starting material. The reaction was then repeated with AgOTf as the Lewis acid. No products could definitively be identified from the complex mixture that resulted, although some signals consistent with oxepine formation were observed in the ${ }^{1} \mathrm{H}$ NMR spectrum. Despite the presence of some promising peaks in the ${ }^{1} \mathrm{H}$ NMR spectrum, the decision was made to investigate the effectiveness of triflate as an alternative leaving group in the reaction. 


\subsubsection{Triflate as the leaving group}

Diol 2.3 was treated with triflic anhydride $\left(\mathrm{Tf}_{2} \mathrm{O}\right)$ in a 1:1 mixture of pyridine (py) and $\mathrm{CH}_{2} \mathrm{Cl}_{2}$ at ambient temperature in an attempt to generate the OTf protected galactal $\mathbf{2 . 6}$ (Scheme 2.6).

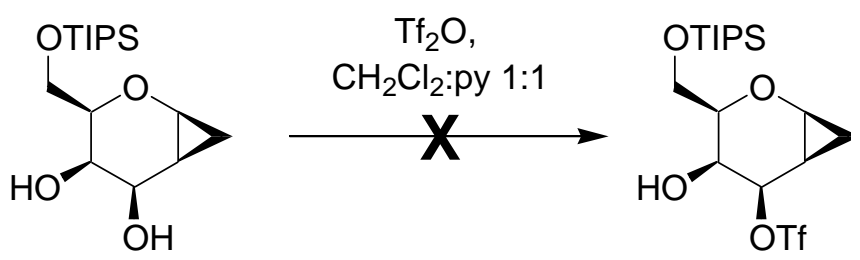

Scheme 2.6. Attempted formation of triflate protected cyclopropane 2.6.

Although none of the expected product was observed an interesting compound was isolated from the reaction mixture. The ${ }^{1} \mathrm{H}$ NMR spectrum revealed the presence of a methylene, indicated by a pair of multiplets at $\delta_{\mathrm{H}} 2.54$, and 2.08 , with a $\delta_{\mathrm{C}}$ of 34.2 . From previous work it is known that ${ }^{1} \mathrm{H}$ NMR signals in this region are consistant with the presence of an oxepine. ${ }^{70}$ Further analysis of the 1D and 2D NMR data indicated the presence of an oxepine with the general structure 2.7. The HSQC-DEPT spectrum revealed the presence of a methylene, one oxymethylene, three oxymethine and two alkenyl carbons which COSY correlations confirmed were all part of the same ${ }^{1} \mathrm{H}$ spin system. The connectivity of the ${ }^{1} \mathrm{H}$ spin system was then established from a series of COSY correlations from the $\mathrm{C}-2$ methylene $\left(\delta_{\mathrm{H}} 2.54\right.$ and $\left.2.08, \delta_{\mathrm{C}} 34.2\right)$ to an oxymethine $\left(\mathrm{C}-1: \delta_{\mathrm{H}} 5.56, \delta_{\mathrm{C}} 100.7\right)$ and to an alkenic methine $\left(\mathrm{C}-3: \delta_{\mathrm{H}} 5.71, \delta_{\mathrm{C}} 124.2\right)$. The proton at $\mathrm{C}-3$ couple to a second alkenic methine $\left(\mathrm{C}-4: \delta_{\mathrm{H}} 6.10, \delta_{\mathrm{C}} 129.1\right)$ to an oxymethine $\left(\mathrm{C}-5: \delta_{\mathrm{H}} 4.58, \delta_{\mathrm{C}} 71.6\right)$ to a second oxymethine $\left(\mathrm{C}-6: \delta_{\mathrm{H}} 4.19, \delta_{\mathrm{C}} 84.8\right)$ and finally to an oxymethylene $\left(\mathrm{C}-7: \delta_{\mathrm{H}} 3.51\right.$ and $\left.3.61, \delta_{\mathrm{C}} 63.5\right)$. This gave an oxepine ring structure 2.7 as shown in Scheme 2.7, where R was an as yet undetermined group. 


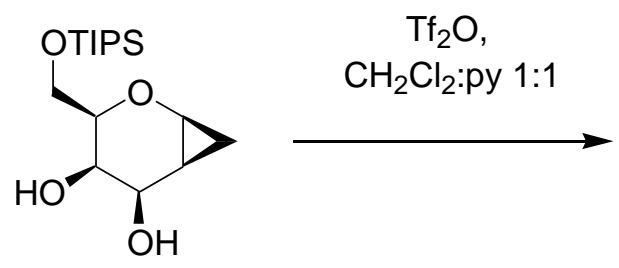

2.3

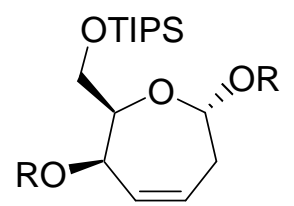

2.7

Scheme 2.7. General structure of oxepine $\mathbf{2 . 7}$ formed from attempted triflate formation.

Initially, it was thought that this compound could have a hydroxyl group at the C-1 position, which could have been formed during the aqueous work up. However, if this were a hemiacetal we would expect to see both anomers present in the NMR spectra, as the ring system could open and close to reach equilibrium between the $\alpha$ - and $\beta$ - forms. In this case, only one isomer was observed. Mass spectral data revealed a $[\mathrm{M}+\mathrm{H}]^{+}$peak at $\sim 299$ that indicated the presence of an oxepine ring with an attached TIPS group. This led us to propose a bicyclic structure for the unknown product 2.7. If the triflate is formed and spontaneously ring expands, the resulting oxonium could be intercepted by an intramolecular nucleophile. The logical source of this nucleophile is the hydroxyl group on the opposite side of the molecule. The oxepine ring is more flexible than the equivalent six-membered ring and so it is possible that the C-5 hydroxyl could reach across the ring and intercept the oxonium ion, forming a bicyclic structure 2.7a as shown in Scheme 2.8.

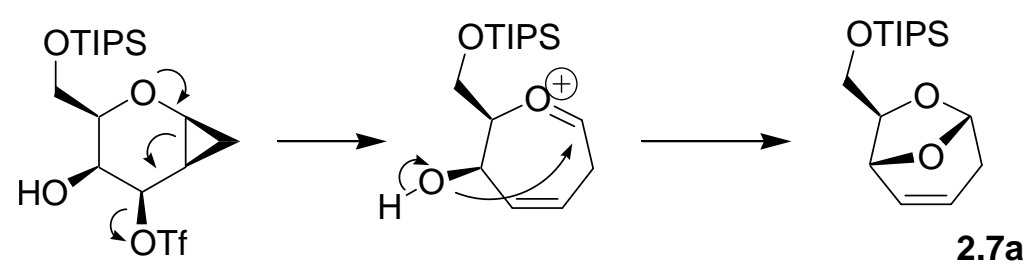

Scheme 2.8. Bicyclic ring formation of 2.7a.

The chemical shifts of the methylene protons in the oxepine ring at C-2 indicate that this could be the correct structure as the two protons are in different chemical 
environments $\left(\delta_{\mathrm{H}} 2.54\right.$ and 2.08), thus indicating a structure that is inflexible on the ${ }^{1} \mathrm{H}$ NMR time-scale.

The low yield in this reaction could be due to some deprotection of the TIPS group from the C-6 hydroxyl. The low molecular weight alcohol formed would probably have a low boiling point and could be lost during the workup step.

Some of the spectral data from compound 2.7 indicated the possible presence of a second TIPS group on the molecule. For instance, the integration of the ${ }^{1} \mathrm{H}$ resonance at $\delta_{\mathrm{H}} 1.09 \mathrm{ppm}$ was twice that expected. It is possible that the molecule has a second OTIPS group present attached at the anomeric position, leading to a structure $\mathbf{2 . 7} \mathbf{b}$ as shown in Figure 2.2. The mass spectral data also suggested that a heavier compound could be present. However, this information was inconclusive because the masses observed did not seem to correspond to likely groups that could be attached to the oxepine.

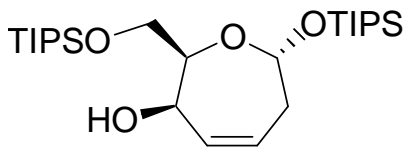

Figure 2.1. Possible structure of product 2.7b from triflation of 2.3.

It is currently considered that the more likely of these two structures is the bicyclic compound 2.7a. The formation of $\mathbf{2 . 7} \mathbf{b}$ would require an OTIPS moiety to be generated and act as a nucleophile, which seems highly unlikely. Nevertheless the alternative structure 2.7b does fit with some of the data obtained from the compound. 


\subsubsection{Mesylate as the leaving group}

An attempt was made to form a mesyl leaving group at C-3. It was thought that it would be possible to isolate the desired cyclopropane product, as the mesyl group should be less labile than triflate during workup. Diol 2.3 was treated with $\mathrm{MsCl}$ in a 1:1 mixture of $\mathrm{CH}_{2} \mathrm{Cl}_{2}$ and pyridine at ambient temperature overnight followed by an aqueous workup. The ${ }^{1} \mathrm{H}$ NMR spectrum of the crude reaction mixture revealed the presence of the previously isolated oxepine $\mathbf{2 . 7}$ along with starting material, and this was not pursued further.

\subsubsection{One pot generation of the triflate leaving group and ring expansion}

The previous reaction had demonstrated that it was indeed possible to generate the triflate leaving group in solution. Subsequent addition of a nucleophile could lead to the ring expansion and generation of the target oxepine.

Initially, cyclopropane 2.3 was treated with $\mathrm{Tf}_{2} \mathrm{O}$ in $\mathrm{CH}_{2} \mathrm{Cl}_{2}$ with an excess of pyridine at $0{ }^{\circ} \mathrm{C}$. After stirring for one hour to form the triflate intermediate, $\mathrm{TMSN}_{3}$ was added drop-wise and the reaction stirred for 12 hours at ambient temperature. Workup of the mixture returned only starting material plus a small amount of oxepine 2.7. Changing the solvent to $\mathrm{MeCN}$ under similar conditions again revealed starting material and oxepine 2.7. Similarly, the employment of TMSallyl as the nucleophile source under identical conditions gave no indication of the formation of the target oxepine and resulted again in the formation of small amounts of oxepine 2.7. An equivalent reaction employing $\mathrm{MeCN}$ as the solvent also failed to generate the desired products. 
At this point it was decided to discontinue the use of these leaving groups. There appeared to be no control over the products of the reactions, and it had proved impossible to introduce an external nucleophile reactive enough to out-compete the internal nucleophile present as a hydroxyl in the molecule at C-5.

\subsection{In situ generation of the leaving group}

The above reactions, despite being technically unsuccessful in generating the protected and activated cyclopropanated galactal, demonstrated the possibility of generating a good leaving group and ring expanding the cyclopropane in situ (Scheme 2.9). This would be a useful modification of the ring expansion method requiring fewer synthetic steps. However, the reaction conditions would have to be optimised so that the external nucleophile would react faster with the oxonium intermediate than the C-5 hydroxyl group.

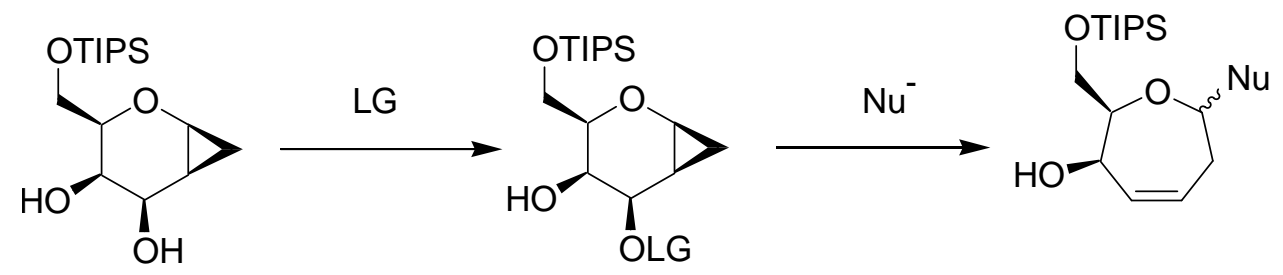

Scheme 2.9. General proposed route to oxepines by in situ generation of a leaving group.

\subsubsection{In situ generation of leaving group under Mitsunobu conditions}

As the initial investigations had revealed that the reaction conditions involving Lewis acid species were too harsh for the TIPS protecting group, conditions were required that would be more conducive to its retention during the reaction. Examination of the literature revealed that the Mitsunobu reaction could possibly be adapted to this 
purpose. $^{73}$ The Mitsunobu reaction is normally used to activate secondary alcohols to attack by nucleophiles so as to introduce other functional groups in a stereoselective manner (Scheme 2.10). ${ }^{74}$ This makes the Mitsunobu reaction an extremely useful tool in natural product synthesis. ${ }^{75}$

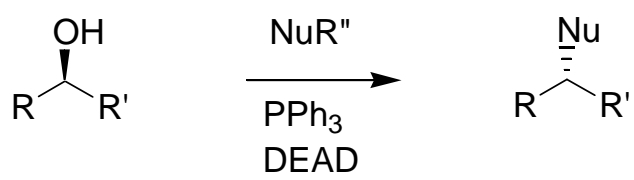

Scheme 2.10. Mitsunobu reaction for conversion of an alcohol to a different functional group.

In the mechanism, $\mathrm{PPh}_{3}$ combines with $\mathrm{DEAD}$ to generate a phosphonium intermediate that subsequently is transferred to the alcohol oxygen, activating it as a leaving group (Scheme 2.11). Substitution of the triphenylphosphonium alkoxide by a suitable nucleophile completes the reaction with inversion of stereochemistry.

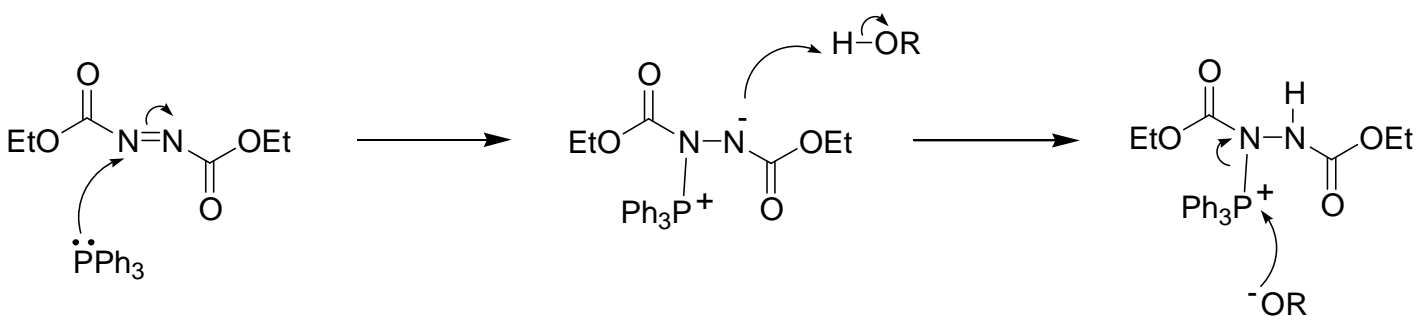

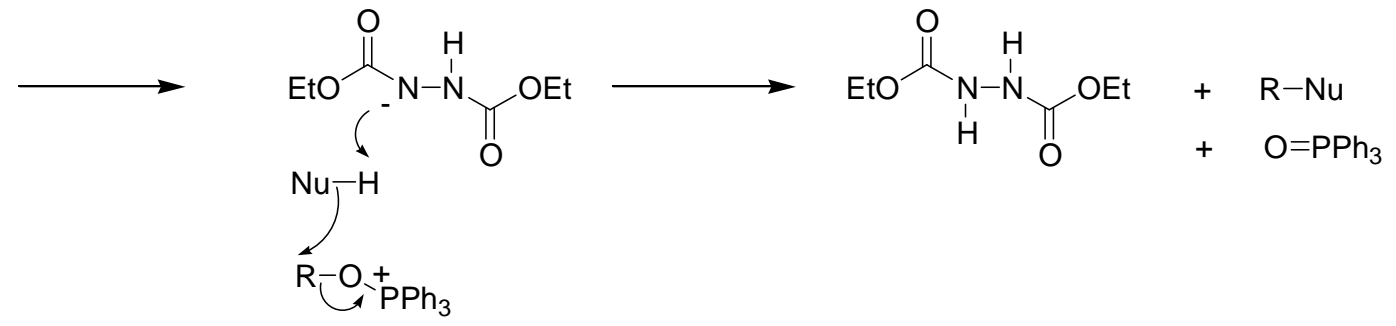

Scheme 2.11. Proposed mechanism of the Mitsunobu reaction.

The intention was to investigate the possibility of using a modification of this reaction to generate a good leaving group that could in turn lead to ring expansion and interception by a nucleophile (Scheme 2.12). 


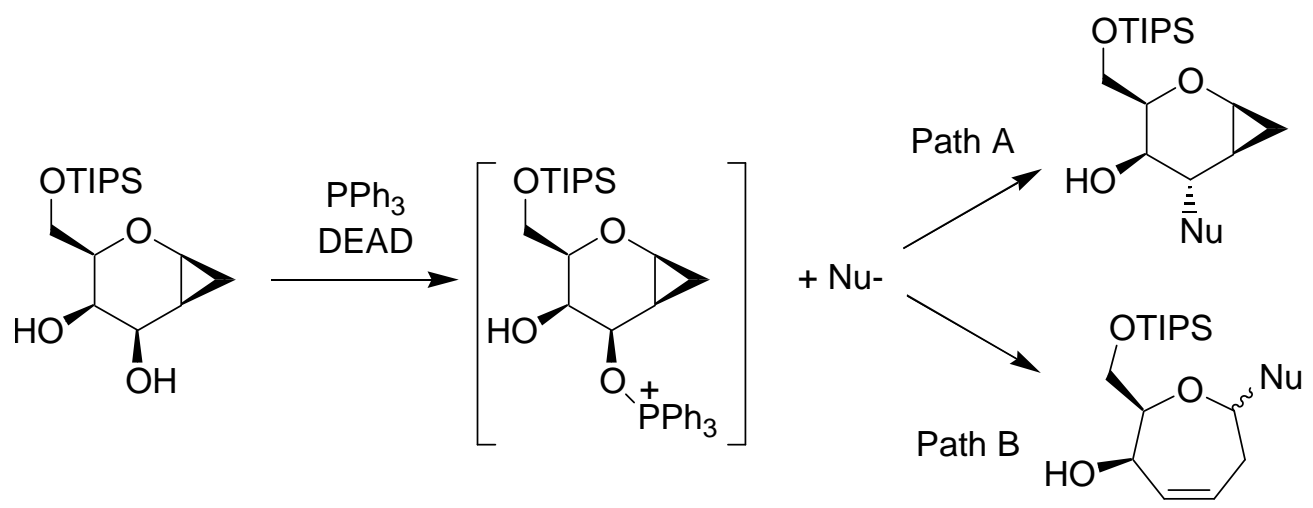

Scheme 2.12. Possible products from Mitsunobu reaction.

There is another product possible from this reaction. The standard Mitsunobu product would be formed if the nucleophile attacks in an $\mathrm{S}_{\mathrm{N}} 2$ or $\mathrm{S}_{\mathrm{N}} 2$ '-type reaction with direct displacement of the triphenylphosphine oxide group by the incoming nucleophile instead of ring expansion. It was thought that if conditions conducive to an $S_{N} 1$ reaction mechanism could be generated, this would lead to the desired opening of the cyclopropane upon departure of the leaving group. The oxonium ion formed in this expansion could then be intercepted by the nucleophile to generate the desired oxepine. This would require a solvent that could stabilise the oxonium intermediate in solution. Hoberg's work suggested that $\mathrm{MeCN}$ was an ideal solvent for this task (Section 1.7). ${ }^{70}$

The first reaction undertaken was to check that the TIPS protecting group at C-6 on cyclopropane 2.3 was stable under the reaction conditions that were to be employed, as this was considered to have been a problem in the previous section's work. Thus cyclopropane 2.3 in THF was treated with $\mathrm{PPh}_{3}, \mathrm{TMSN}_{3}$ and DEAD at $0{ }^{\circ} \mathrm{C}$. This was allowed to stir for 12 hours warming to ambient temperature. Work up of the reaction mixture followed by purification gave pyran 2.9 as one isomer in an $82 \%$ yield (Scheme 2.13). 


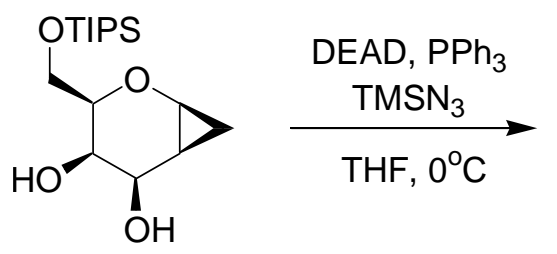

2.3

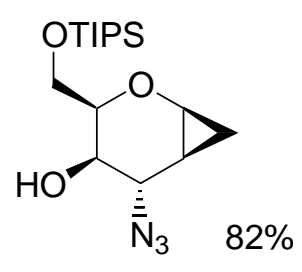

2.9

Scheme 2.13. Mitsunobu reaction in THF on $\mathbf{2 . 3}$ with $\mathrm{TMSN}_{3}$ as the nucleophile to generate 2.9.

This result (entry 1, Table 2.1) demonstrated both the stability of the TIPS group under the reaction conditions, and that the nucleophile also functioned given the constraints of the reaction. ${ }^{76-78}$

For the next reaction the solvent was altered to one that was intended to favour the desired ring expansion. $\mathrm{MeCN}$ was chosen for the reasons listed previously, and as it is known to assist the stereo- and regio-selective glycosylation of carbohydrates by forming an ion pair with the ring oxygen and directing the incoming nucleophile. ${ }^{79}$ Thus cyclopropane 2.3 in MeCN was treated with $\mathrm{PPh}_{3}, \mathrm{TMSN}_{3}$ and DEAD at $0{ }^{\circ} \mathrm{C}$. Workup generated the non-rearranged product 2.9 with azide substitution at the C-3 position in a $10 \%$ yield in a 1:1 yield of epimers, along with return of the starting material (Scheme 2.14). A subsequent literature search revealed that $\mathrm{MeCN}$ has also been used as a solvent in the Mitsunobu reaction. ${ }^{80}$

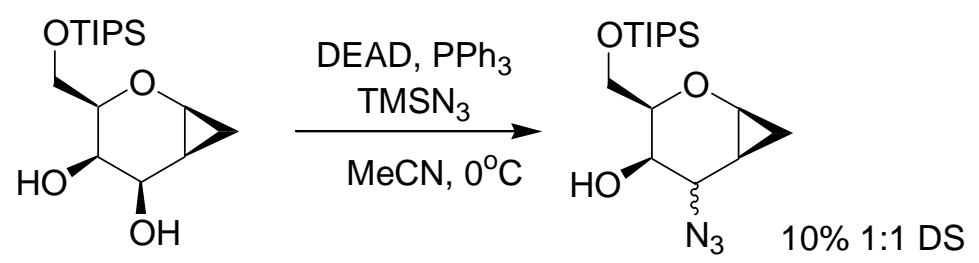

Scheme 2.14. Mitsunobu reaction in $\mathrm{MeCN}$ with $\mathrm{TMSN}_{3}$ as the nucleophile.

This result will be discussed later (entry 2, Table 2.1). It was then decided to alter the nucleophile to TMSallyl for the next reaction to examine the effect of using a C-nucleophile verses an $\mathrm{N}$-nucleophile. The solvent was altered to $\mathrm{CH}_{2} \mathrm{Cl}_{2}$ as $\mathrm{MeCN}$ 
had not yielded the desired ring expansion. Cyclopropane 2.3 in $\mathrm{CH}_{2} \mathrm{Cl}_{2}$ was treated with $\mathrm{PPh}_{3}$, TMSallyl and DEAD at $0{ }^{\circ} \mathrm{C}$. The ${ }^{1} \mathrm{H}$ NMR spectrum of the crude reaction mixture revealed that some oxepine had been formed. This was purified and assigned using 1 and 2D NMR. An examination of the ${ }^{1} \mathrm{H}$ NMR spectrum revealed that there were two epimers present as a 1:1 mixture. No signals indicative of an allyl group were observed. However the ${ }^{1} \mathrm{H}$ NMR spectrum indicated that the TIPS group was still present on the molecule. An HSQC-DEPT revealed the presence of two epimeric hemiacetal ${ }^{1} \mathrm{H}$ signals $\left(\delta_{\mathrm{H}} 5.41\right.$ and $\left.5.10 \mathrm{ppm}\right)$ and the corresponding anomeric hydroxyl signals $\left(\delta_{\mathrm{H}} 3.38\right.$ and $\left.3.00 \mathrm{ppm}\right)$. COSY NMR confirmed the oxepine ring connectivity and indicated that $\mathbf{2 . 1 0}$ was the reaction product, obtained in a $17 \%$ yield (Scheme 2.15).

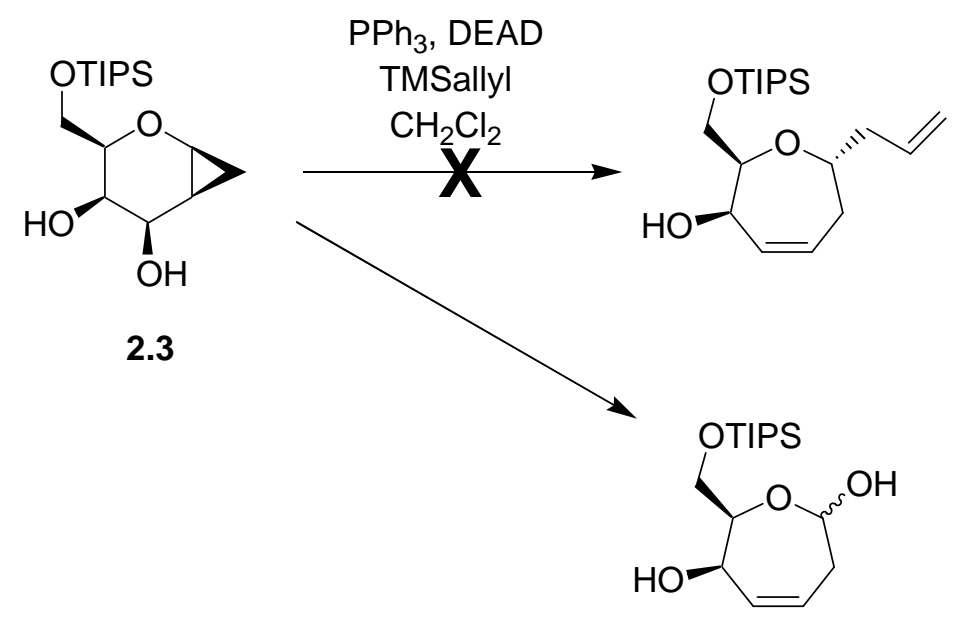

2.10

Scheme 2.15. Reaction of $\mathbf{2 . 3}$ under Mitsunobu conditions with TMSallyl as nucleophile.

This result indicated that while the oxonium intermediate had been formed, there was no observable interception by the allyl nucleophile. The presence of the previously observed 2.7 was not observed. It is thought that the formation of the bridged species is not facilitated by the $\mathrm{CH}_{2} \mathrm{Cl}_{2}$ and the hemiacetal $\mathbf{2 . 1 0}$ could be formed if the oxonium ion is quenched during the aqueous workup. 
The next solvent selected to try the reaction in was DMF. It was thought that the presence of the oxygen and its associated lone pairs of electrons would stabilise the oxonium cation in solution more effectively than $\mathrm{MeCN}$ assisted by the lone pair of electrons on the nitrogen (Scheme 2.16).

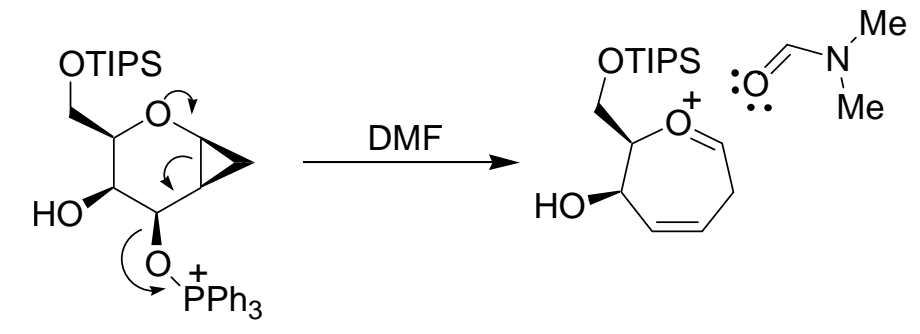

Scheme 2.16. Stabilisation of the oxonium intermediate by DMF.

Thus cyclopropane 2.3 was treated in DMF with $\mathrm{TMSN}_{3}, \mathrm{PPh}_{3}$ and DEAD at $0{ }^{\circ} \mathrm{C}$ and the reaction stirred for two hours. Work up and purification of the reaction gave the desired oxepine $\mathbf{2 . 1 1}$ as the only compound recovered in a $27 \%$ yield (Scheme 2.17). The selectivity was determined by GC to be $4: 1$, with the major epimer assumed to be the $\alpha$ anomer based on precedence from the Ferrier rearrangement with glycals.

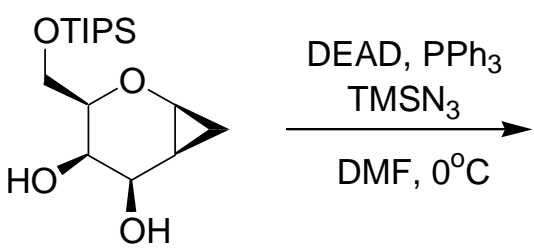

2.3

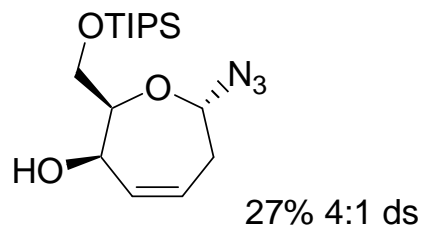

2.11

Scheme 2.17. Modified Mitsunobu reaction in DMF at $0{ }^{\circ} \mathrm{C}$ with $\mathrm{TMSN}_{3}$ as the nucleophile.

In an attempt to improve the selectivity and yield of the reaction, it was then decided to lower the temperature to $-20{ }^{\circ} \mathrm{C}$. This gave $\mathbf{2 . 1 1}$ again as the only compound recovered in a $45 \%$ yield with a stereoselectivity by GC of 20:1 (Scheme 2.18). 


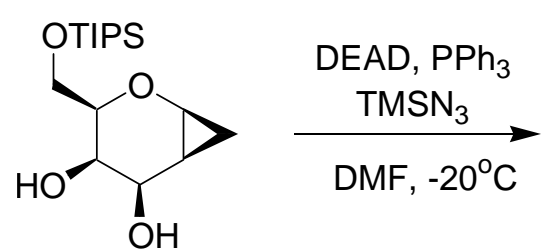

2.3

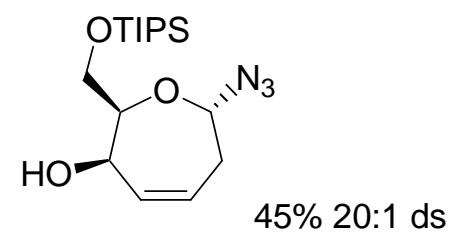

2.11

Scheme 2.18. Modified Mitsunobu reaction in DMF at $-20{ }^{\circ} \mathrm{C}$ with $\mathrm{TMSN}_{3}$ as the nucleophile.

This increase in yield and selectivity prompted a further experiment, lowering the temperature of the reaction to $-40{ }^{\circ} \mathrm{C}$. Workup in this case revealed no oxepine. Instead the major product isolated was the azide $\mathbf{2 . 9}$ in $87 \%$ yield in a 2:1 ratio of epimers by GC at C-3 (Scheme 2.19). These reactions are summarised in Table 2.1.<smiles>O[C@H]1[C@@H]2CC2OC(CO[SnH3])[C@@H]1O</smiles>

2.3

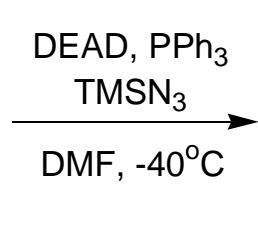

Scheme 2.19. Modified Mitsunobu reaction in DMF at $-40{ }^{\circ} \mathrm{C}$ with $\mathrm{TMSN}_{3}$ as the nucleophile.

The results suggest that the mechanism for the reaction is temperature and solvent dependent. With the reactions run in DMF at $0{ }^{\circ} \mathrm{C}$ we see the formation of the $\alpha$ oxepine, but with a substantial amount (20\%) of the $\beta$-product present (entry 3). Decreasing the temperature to $-20{ }^{\circ} \mathrm{C}$ further decreases the amount of the $\beta$-product formed ( $\sim 5 \%)$ and increases the yield (entry 4$)$. At $-40{ }^{\circ} \mathrm{C}$ there appears to be sufficient energy for the leaving group to depart but not for the opening of the cyclopropane (entry 5).

Thus a cation is formed at C-3 that is stabilised by DMF at the lower reaction temperature and this is then intercepted by the nucleophile (Scheme 2.20). The moderate $\alpha$-selectivity observed in entry 3 is possibly due to the stabilising DMF 
molecule weakly H-bonding to the adjacent hydroxyl at C-4 slightly hindering attack from the $\beta$-face. This stabilisation effect does not appear to occur with $\mathrm{MeCN}$ as the solvent (entry 2) for either the ring expansion or the substitution at C-3.

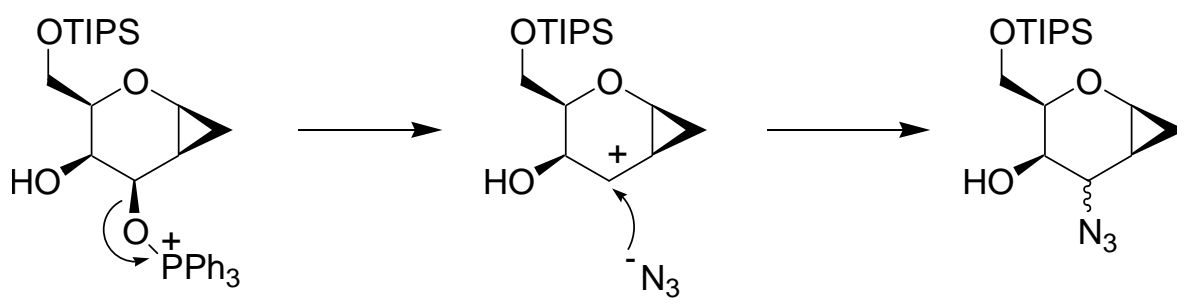

Scheme 2.20. Possible mechanism for formation of azide 2.9.

Table 2.1. Reaction of cyclopropane 2.3 under modified Mitsunobu conditions.

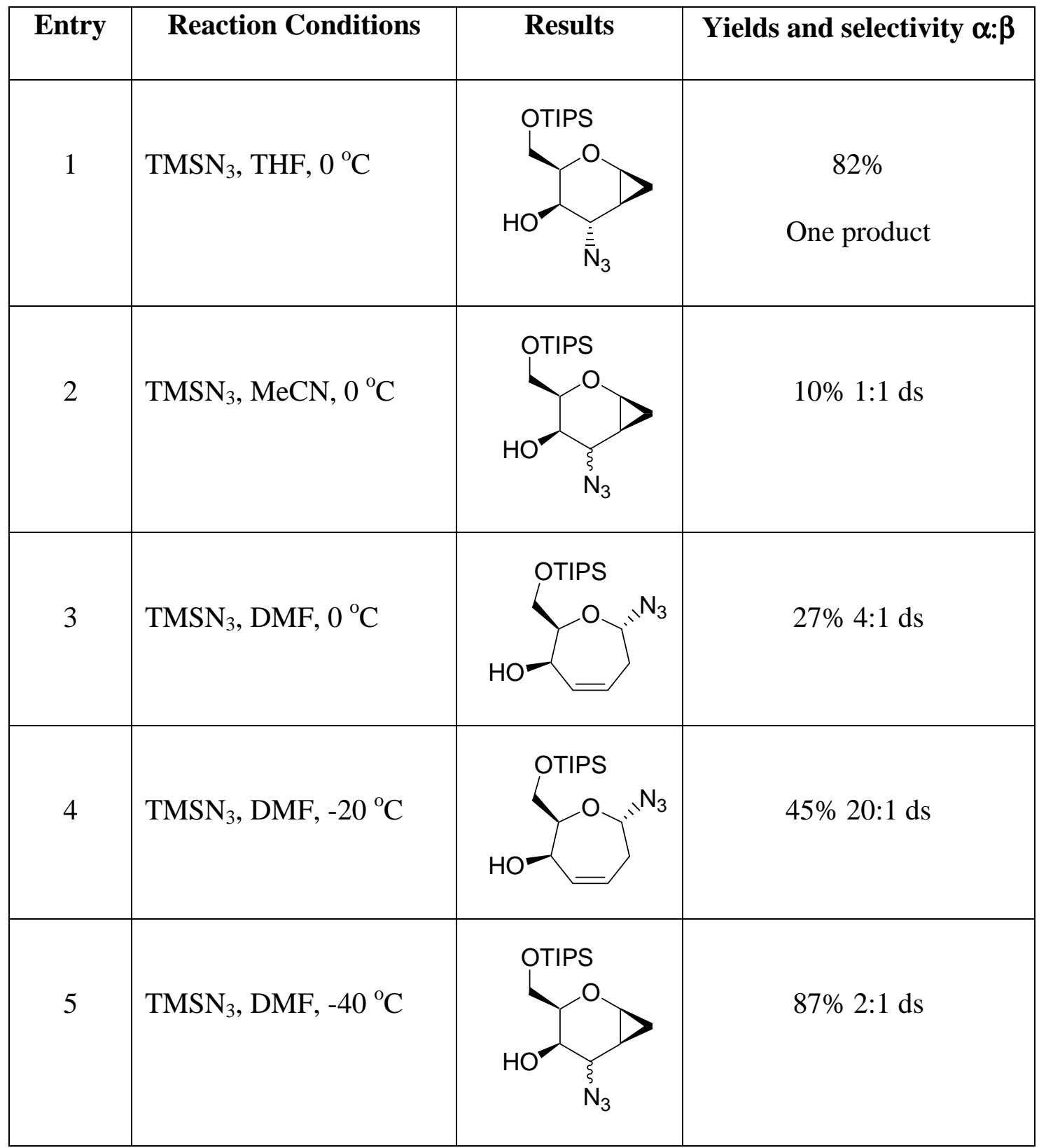


At this point it was decided to halt this line of investigation utilising a monotethered silyl protecting group due to its instability under either the Lewis acid or Mitsunobu expansion conditions. While some initially promising results had been observed, the yields were lower than desired, and the reaction could not easily be controlled. The presence of unexpected by-products was also a concern.

\subsection{Ring expansion of glucal 2.12 with generation of leaving group in} situ

Investigations to be discussed in Chapter six suggested the possibility that the leaving group at C-3 could be generated in situ. This was previously investigated as described in Section 2.3, but could not be successfully controlled. Therefore a test reaction was

performed on cyclopropane $\mathbf{2 . 1 2}$ (as previously synthesized by Hoberg) ${ }^{70}$ without the acetate present. Excess TMSOTf was used and it was intended that trimethylsilylation of the hydroxyl group at C-3 would generate a sufficiently good leaving group in situ for the reaction to proceed. It was thought that the di-tert-butylsilyl protecting group would be stable even under the acidic conditions that would result from this reaction. Thus cyclopropane $\mathbf{2 . 1 2}$ was treated with 1.2 equivalents of TMSOTf and five equivalents of $\mathrm{TMSN}_{3}$ in acetonitrile (Scheme 2.21).

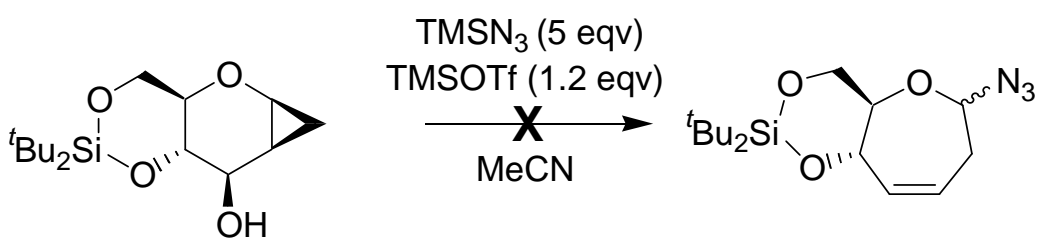

Scheme 2.21. Attempted ring expansion of $\mathbf{2 . 1 2}$ generating the leaving group in situ.

This generated three products which were separated by column chromatography on silica. They were characterized in order of elution from the silica column. All 
structures were assigned by ${ }^{1} \mathrm{H}$ and ${ }^{13} \mathrm{C}$ NMR, COSY, HSQC-DEPT, and where avaliable $\mathrm{HMBC}$ and ${ }^{15} \mathrm{~N}$ experiment.

The compound with the highest $\mathrm{R}_{\mathrm{F}}$ had ${ }^{1} \mathrm{H}$ resonances that indicated the presence of the di-tert-butylsilyl protecting group $\left(\delta_{\mathrm{H}} 1.07,9 \mathrm{H}\right.$ and $\left.\delta_{\mathrm{H}} 1.01,9 \mathrm{H}\right)$. Analysis of the HSQC-DEPT data indicated the presence of an oxymethylene, two oxymethines, two alkenic methines and a methylene group. There was also a quaternary carbon present. The connectivity of the ${ }^{1} \mathrm{H}$ spin system was constructed from a series of COSY correlations from the C-7 oxymethylene $\left(\delta_{\mathrm{H}} 3.83\right.$ and $\left.\delta_{\mathrm{H}} 3.99, \delta_{\mathrm{C}} 68.5\right)$ to an oxymethine $\left(\mathrm{C}-6: \delta_{\mathrm{H}} 3.53, \delta_{\mathrm{C}} 70.5\right)$ to a second oxymethine $\left(\mathrm{C}-5: \delta_{\mathrm{H}} 4.50, \delta_{\mathrm{C}} 75.1\right.$,) to an alkenic methine $\left(\mathrm{C}-4: \delta_{\mathrm{H}} 5.67, \delta_{\mathrm{C}} 135.5\right)$ to a second alkenic methine $\left(\mathrm{C}-3: \delta_{\mathrm{H}} 5.60\right.$, $\left.\delta_{\mathrm{C}} 120.2\right)$ to a methylene $\left(\mathrm{C}-2: \delta_{\mathrm{H}} 3.33\right.$ and $\left.\delta_{\mathrm{H}} 3.26, \delta_{\mathrm{C}} 16.7\right)$. The quaternary carbon was assumed to be at C-1 $\left(\delta_{\mathrm{C}} 118.1\right)$ as all other resonances had been identified. The chemical shift indicated that $\mathrm{C}-1$ was olefinic, most likely with a nitrogen from the azide addition. The complexity of the ${ }^{1} \mathrm{H}$ splitting at the $\mathrm{C}-2$ methylene indicated that the oxepine ring was intact. A TMS group was present in the sample $\left(\delta_{\mathrm{H}} 0.12, \delta_{\mathrm{C}} 0.14\right)$, which integrated for nine protons. ES HRMS data was obtained for this compound, with a strong peak in the spectrum occurring at 370.2271 Da. This suggested that the structure of the compound could possibly be as shown in Figure 4.1. The calculated mass for the proposed structure $2.13 \mathrm{C}_{18} \mathrm{H}_{35} \mathrm{O}_{3} \mathrm{NSi}_{2}+\mathrm{H}$ matched that observed. This information indicated that the TMS group observed in the 1D NMR spetra was indeed part of the structure. As no other moieties had been identified that could be attached to the $\mathrm{sp}^{2}$ hybridised nitrogen attached at $\mathrm{C}-1$, the TMS was tentatively placed at this position. Literature ${ }^{13} \mathrm{C}$ chemical shift data for similar systems supported this assignment. ${ }^{81}$ Based on this information the structure of this compound was tentatively assigned as that shown in Figure 2.2. 


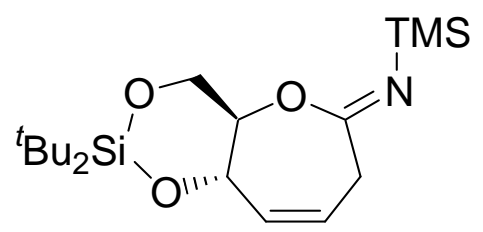

Figure 2.2. Proposed structure of oxepine 2.13.

Unfortunately no further information could be obtained from this compound as by the time more advanced NMR experiments had become available $\left({ }^{15} \mathrm{~N}\right.$ and ${ }^{13} \mathrm{C} \mathrm{HMBC}$, as well as ${ }^{29} \mathrm{Si}$ ) the stored sample had decomposed to an unidentifiable oil.

The compound with the middle $\mathrm{R}_{\mathrm{F}}$ also had ${ }^{1} \mathrm{H}$ resonances indicative of the di-tertbutylsilyl protecting group. Analysis of the HSQC-DEPT spectrum revealed the presence of an oxymethylene, a methylene, three oxymethines and two alkenic methines. The connectivity of the ${ }^{1} \mathrm{H}$ spin system was constructed from a series of COSY correlations from the C-7 oxymethylene $\left(\delta_{\mathrm{H}} 4.15\right.$ and $\left.3.86, \delta_{\mathrm{C}} 67.8\right)$ to an oxymethine $\left(\mathrm{C}-6: \delta_{\mathrm{H}} 3.58, \delta_{\mathrm{C}} 69.7\right)$ to a second oxymethine $\left(\mathrm{C}-5: \delta_{\mathrm{H}} 4.53, \delta_{\mathrm{C}} 74.5\right)$ to an alkenic methine $\left(\mathrm{C}-4: \delta_{\mathrm{H}} 5.67, \delta_{\mathrm{C}} 134.0\right)$ to a second alkenic methine $\left(\mathrm{C}-3, \delta_{\mathrm{H}} 5.67, \delta_{\mathrm{C}}\right.$ 126.1) to a methylene $\left(\mathrm{C}-2: \delta_{\mathrm{H}} 2.59 \delta_{\mathrm{C}} 33.1\right)$ and finally to an oxymethine $\left(\mathrm{C}-1, \delta_{\mathrm{H}} 4.81\right.$, $\delta_{\mathrm{C}}$ 77.3). This gave a base structure as shown in Figure 2.3.

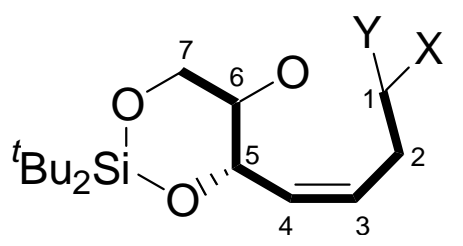

Figure 2.3. Substructure of middle $\mathrm{R}_{\mathrm{F}}$ compound generated from ring expansion with leaving group generated in situ.

Fortunately this sample had not decomposed during storage and it was possible to run more advanced 2D NMR experiments. ${ }^{15} \mathrm{~N}$ HMBC experiments indicated the presence of an azide, which was confirmed by a peak in the IR spectrum at $2099 \mathrm{~cm}^{-1}$. The ${ }^{15} \mathrm{~N}$ 
shifts of the three nitrogens were successively $\delta_{N}=-292,-136$ and -302 . Interestingly there was also a fourth peak present at $\delta_{\mathrm{N}}-155$. However, the fact that this was coupled to a methine was initially rather confusing. ${ }^{13} \mathrm{C}$ HMBC correlations indicated that there was no C-6 to C-1 ether linkage and hence no oxepine ring. This was backed up by the resonance of the C-2 methylene in which both protons were chemically equivalent by ${ }^{1} \mathrm{H}$ NMR. This had not been observed in any of the oxepine ring systems synthesised as part of this project, or in Hoberg's previous work, ${ }^{70}$ and indicated that there was free rotation around this centre on the NMR timescale. The chemical shift of the methine suggested that there was more than one heteroatom present at this centre. The presence of a tetrazole was discounted, as the $\mathrm{C}-1$ in the ring would give ${ }^{13} \mathrm{C}$ NMR signals at approximately $\delta_{\mathrm{C}} \sim 140-170$ according to literature sources, ${ }^{82}$ whereas a signal at $\delta_{\mathrm{C}} 77.3$ was observed for this centre. Another possibility was the presence of a diazide at this centre. Similar compounds have previously been reported in the literature, for example Rydzewski and coworkers generated a diazide under similar conditions (Scheme $2.22){ }^{83}$

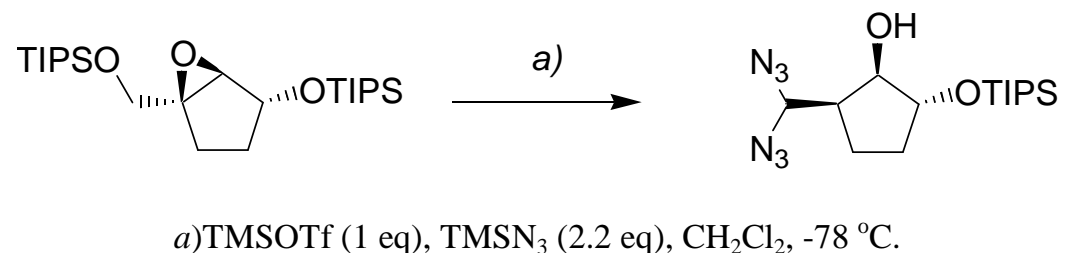

Scheme 2.22. Rydzewski's diazide synthesis.

The chemical shifts of the diazide methine in this compound $\left(\delta_{\mathrm{H}} 4.78, \delta_{\mathrm{C}} 78.7\right)$ very closely matched those of C-1 in the unknown sample. Unfortunately no ES HRMS data could be obtained for our compound to confirm the structure. However, given the data we possessed it was possible to propose the structure $\mathbf{2 . 1 4}$ for this compound (Figure 2.4). 


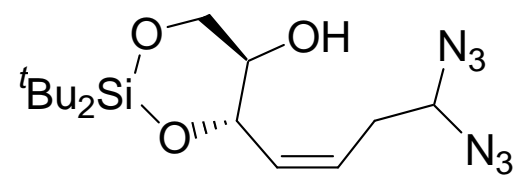

Figure 2.4. Probable structure of diazide 2.14.

More evidence for diazide $\mathbf{2 . 1 4}$ was the presence of a fourth peak in the ${ }^{15} \mathrm{~N}$ spectrum at $\delta_{\mathrm{N}}-155$. This signal and the signal at $\delta_{\mathrm{N}}-136$ correspond to the chemical shift range expected for the second positively charged nitrogen on the azide chain. ${ }^{15} \mathrm{~N}$ data on the oxepine azides 3.5 and 3.17 synthesised in chapter three agrees with these chemical shifts, but the second nitrogen occurs at $\delta_{\mathrm{N}}-135.8$. The presence of the extra peak at $\delta_{\mathrm{N}}-155 \mathrm{ppm}$ is thought to be due to a weak interaction between this central $\mathrm{N}$ of one azide group and one of the lone pairs on the alcohol attached to C-2 (Figure 2.5)

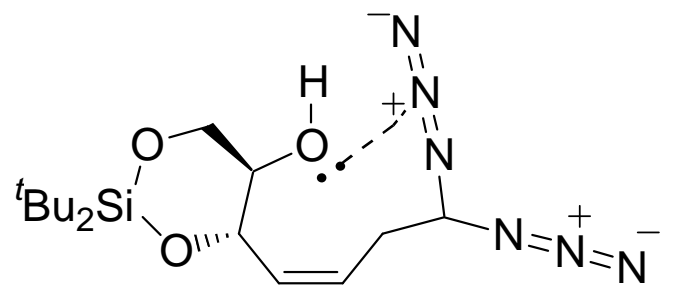

Figure 2.5. Proposed structure of diazide $\mathbf{2 . 1 4}$ showing interaction between positively charged nitrogen and the free alcohol.

The last compound also appeared to have no oxepine ring. Again ${ }^{1} \mathrm{H}$ resonances confirmed the presence of the di-tert-butylsilyl protecting group. The HSQC-DEPT spectrum revealed the presence of an oxymethylene, two oxymethines, two alkenic methines, a methylene and a quaternary carbon. The connectivity of the ${ }^{1} \mathrm{H}$ spin system was constructed from a series of COSY correlations from the C-7 oxymethylene $\left(\delta_{\mathrm{H}}\right.$ 4.11 and $\left.3.85, \delta_{\mathrm{C}} 68.0\right)$ to an oxymethine $\left(\mathrm{C}-6: \delta_{\mathrm{H}} 3.59, \delta_{\mathrm{C}} 69.6\right)$ to a second oxymethine $\left(\mathrm{C}-5: \delta_{\mathrm{H}} 4.51, \delta_{\mathrm{C}} 74.7\right)$ to an alkenic methine $\left(\mathrm{C}-4: \delta_{\mathrm{H}} 5.76, \delta_{\mathrm{C}} 135.4\right)$ to a second alkenic methine $\left(\mathrm{C}-3: \delta_{\mathrm{H}} 5.63, \delta_{\mathrm{C}} 120.8\right)$ to a methylene $\left(\mathrm{C}-2: \delta_{\mathrm{H}} 3.30, \delta_{\mathrm{C}} 16.8\right)$. HMBC correlation revealed that C-2 correlatated to the quaternary carbon at $\mathrm{C}-1\left(\delta_{\mathrm{C}}\right.$ 
118.0). HMBC correlations also revealed no ring ether linkage. ${ }^{1} \mathrm{H}^{-15} \mathrm{~N}$ HMBC revealed the presence of one nitrogen in the molecule. As C-1 was coupled to the C-2 methylene it was then assigned as a nitrile, which was in agreement with the observed C-1 chemical shift data. ${ }^{84}$ A broad singlet at $\delta_{\mathrm{H}} 2.35$ was assigned as originating from a hydroxyl connected to C-6 by HMBC. This data allowed the proposal of the structure 2.15 (Figure 2.6). Final conformation was provided by a peak in the IR spectrum at $2254 \mathrm{~cm}^{-1}$ indicative of the presence of a nitrile group.

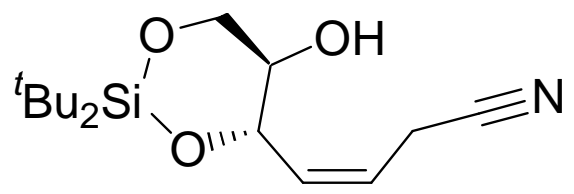

Figure 2.6. Proposed structure for the nitrile $\mathbf{2 . 1 5}$.

In order to explain these products, it is postulated that the reaction proceeds through initial ring expansion to form the azide-substituted oxepine. However, in the presence of excess TMSOTf it is then possible for subsequent reactions to occur. It is postulated that the observed products $2.13,2.14$ and 2.15 are formed through two competing pathways (Scheme 2.23).

In pathway $\mathrm{A}$, it is proposed that a lone pair of electrons on the oxepine ring oxygen attacks the TMS group which allows tautomerisation of the azide. This rearrangement is reversible. A second azide nucleophile then attacks at $\mathrm{C}-1$, breaking the $\mathrm{C}-\mathrm{O}$ ether bond and opening the oxepine ring, generating the diazide 2.14. In pathway B, elimination of $\mathrm{N}_{2}$ from the azide coupled with protonation at C-1 (possibly aided by triflate) generates a $\mathrm{C}-\mathrm{N}$ double bond to give oxepine 2.13. Compound 2.13 can then react further through protonation of the oxepine ring oxygen and ring opening with elimination of the $\mathrm{N}$ bound TMS group to give nitrile $\mathbf{2 . 1 5}$. 


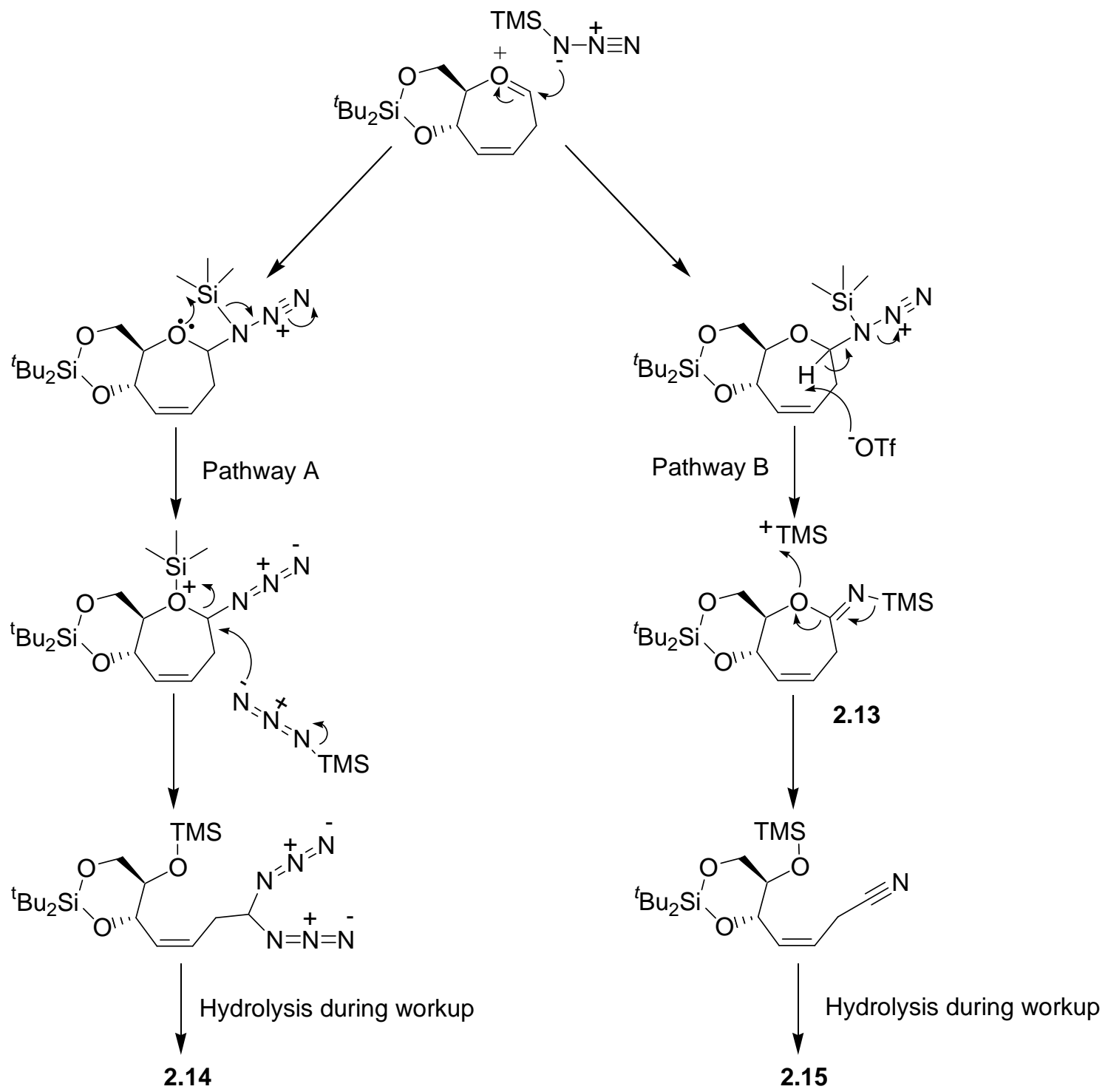

Scheme 2.23. Rearrangement of oxepine 2.12 with $\mathrm{TMSN}_{3}$ in TMSOTf to give observed reaction products.

\subsection{Investigation of intramolecular nucleophile delivery}

It had been observed in our previous work that an internal nucleophile reacts faster than an external nucleophile with the oxonium intermediate. Therefore it might be possible to utilise a cyclopropanated glycal with an internal nucleophilic moiety to intercept the oxonium intermediate and form an oxepine. The decision was also made to retain the 
use of the di-tert-butylsilyl protecting group due to its stability under a wide range of reaction conditions.

\subsubsection{The tandem Tebbe-Claisen rearrangement}

Fraser-Reid and co-workers have reported Claisen-type rearrangements of glycals in which the enol ether at $\mathrm{C}-3$ rearranges at high temperature to effectively become the nucleophile at $\mathrm{C}-1^{85}$ (Scheme2.21).

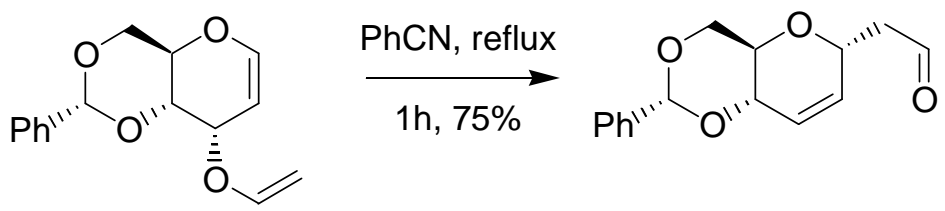

Scheme 2.24. Fraser-Reid's Claisen approach to $C$-alkylation of glycals.

Fairbanks and co-workers expanded on this work with substituted enol ethers in the synthesis of $C$-glycosides using a tandem Tebbe-Claisen approach (Scheme 2.25). ${ }^{86}$

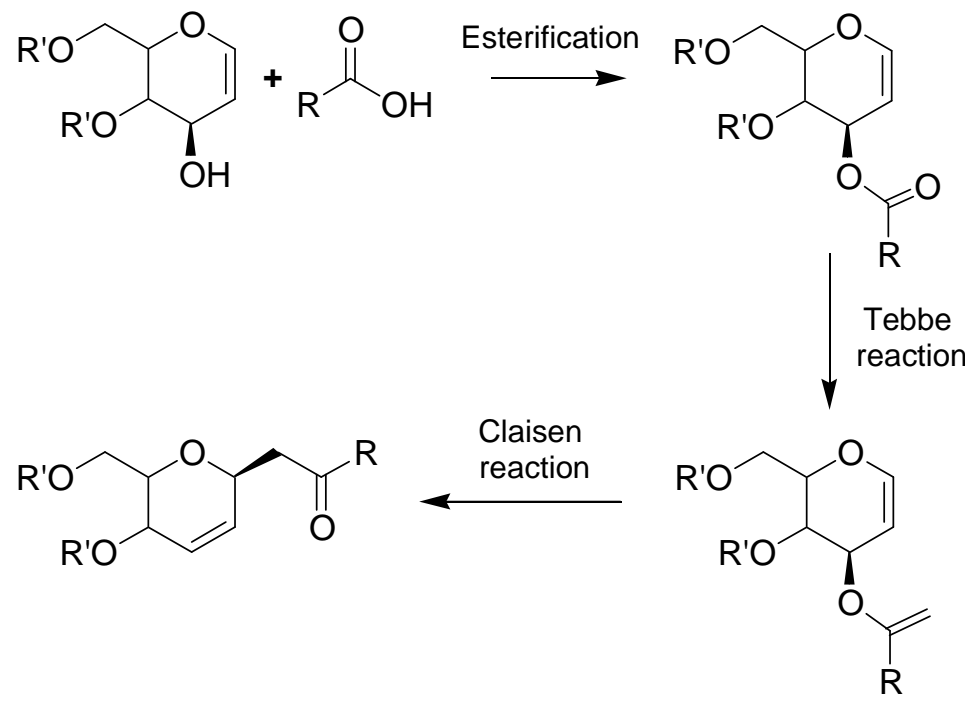

Scheme 2.25. Tebbe-Claisen approach to formation of $C$-glycosides. 
This approach involves the esterification of a suitable glycal ester. Tebbe methylenation produces an enol ether that undergoes a stereoselective sigmatropic rearrangement to yield the desired $C$-glycoside. The Tebbe reagent is formed by the reaction of $\mathrm{Cp}_{2} \mathrm{TiCl}_{2}$ with $\mathrm{AlMe}_{3}$ (Scheme 2.26). This produces an organometallic species that can then react with a carbonyl group to produce an olefin by the transfer of a methylene group. ${ }^{64}$
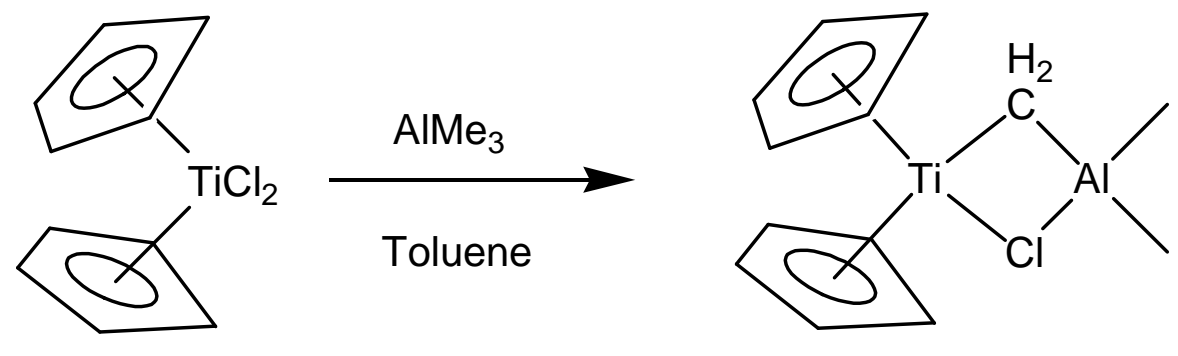

Tebbe's reagent

Scheme 2.26. Formation of Tebbe's reagent.

The Tebbe reaction is especially useful in reactions with ester carbonyl groups that are unreactive under standard Wittig conditions. The advantages of Fairbanks' approach are the wide selection of acids that may be used in the initial esterification (including amino acids) ${ }^{87}$ and the stereoselectivity of the $\mathrm{C}-\mathrm{C}$ bond formation, in which the anomer produced depends on the initial glycal selected. Our interest was centred on the application of this methodology to the cyclopropane ring expansion. By modifying Fairbanks reaction it should prove possible to access a range of ring expanded compounds with $\beta$-substitution that our current strategy could not produce with high selectivity. It would also allow the use of a far wider range of nucleophiles than had been previously possible.

A long-term goal would be to create oxepane nucleosides analogous to the HIV drug AZT (Figure 2.7) in which an oxepane replaces the furan ring. 


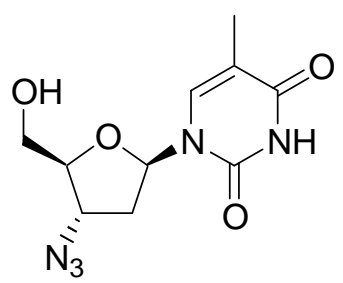

Figure 2.7. Structure of AZT.

These oxepanes have very recently been reported by Damha and Sabatino. ${ }^{88}$ Ring expansion of glucal $\mathbf{1 . 6 1}$ with a silylated nucleoside base (thymine or $\mathrm{N}^{6}$-benzoyladenine) and TMSOTf in refluxing MeCN gave the desired oxepine in approximately $40 \%$ yield as a 1:1 mixture of anomers. The desired $\beta$-anomer was separated from the remainder of the mixture and converted to the desired oxepane by treatment with TBAF, followed by hydrogenation over Pd/C (Scheme 2.27).<smiles>Cc1cn([C@H]2CCC[C@@H](O)[C@@H](CO)O2)c(=O)[nH]c1=O</smiles>

a) TMSthymine, TMSOTf, MeCN. b)i) TBAF. ii) $\mathrm{H}_{2} / \mathrm{Pd}$.

Scheme 2.27. Damha and Sabatino's formation of an oxepane nucleoside.

A perceived problem with the application of this type of reaction to our system was that the cyclopropane was blocking access to the $\beta$-face of the $\mathrm{C}-1$ carbon. With a true Claisen pericyclic reaction less likely, there is a possibility that a $\mathrm{S}_{\mathrm{N}} 1$ type mechanism could occur leading to a mixture of epimers. However it was felt that the potential benefits if the reaction was successful made it worth investigating. 


\subsubsection{Ring expansion using modified Tebbe-Claisen}

Using cyclopropanated glucal $\mathbf{1 . 6 1},{ }^{70}$ the acetate protecting group at C-3 was methylenated with Tebbe's reagent to give olefin 2.15. This reaction was low yielding and it proved difficult to purify the products as there were problems removing the titanocene oxide by-product. The Claisen rearrangement was attempted in several different solvents and various temperatures following the previously reported work. Rearrangment products were isolated but proved to be a 1:1 mixture of epimers of $\mathbf{2 . 1 6}$ as determined by ${ }^{1} \mathrm{H}$ and HSQC-DEPT NMR (Scheme 2.25) in a $45 \%$ yield.

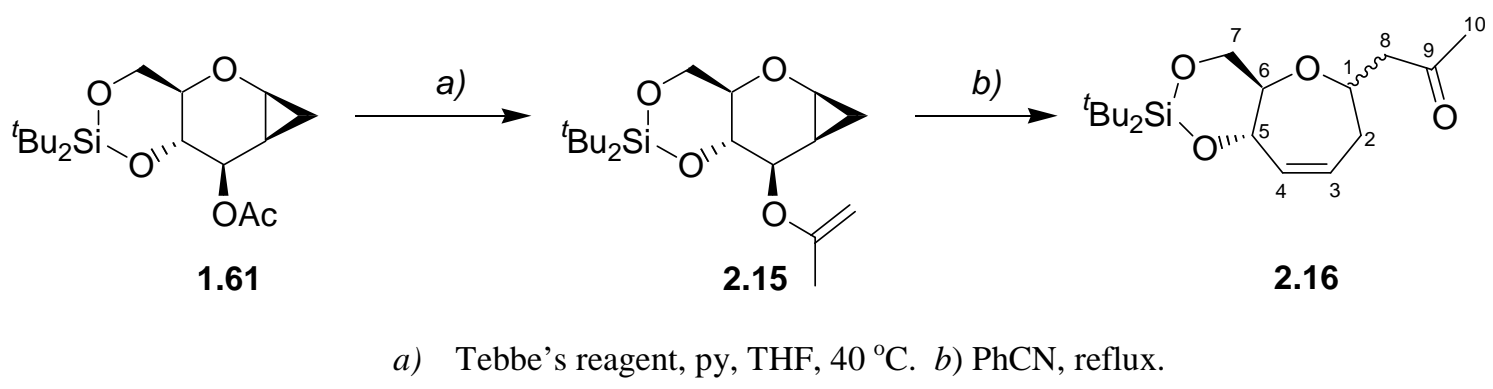

Scheme 2.28. Rearrangement of $\mathbf{1 . 6 1}$ by tandem Tebbe-Claisen rearrangement to form $\mathbf{2 . 1 6}$.

The mixture of isomer observed in the products may result from the high temperatures leading to an $\mathrm{S}_{\mathrm{N}} 1$ type reaction where the group at $\mathrm{C}-3$ leaves and rearrangement occurs, which leads to the formation of the oxonium intermediate. The so-formed acetone enolate then attacks from either face at C-1 (Scheme 2.29). This had been considered a possible outcome at the outset of this section.

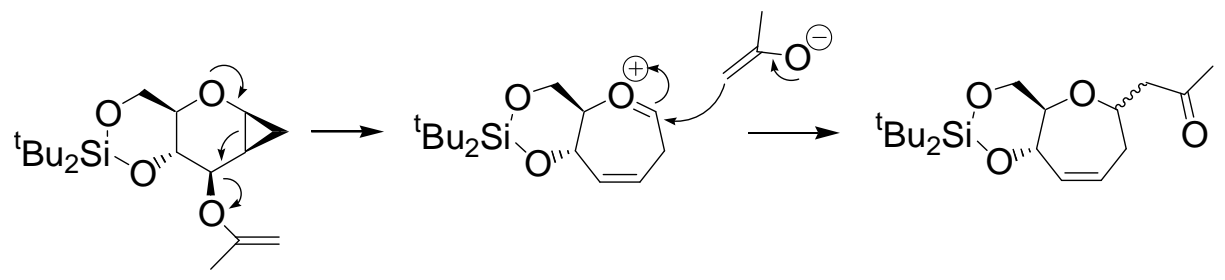

Scheme 2.29. Possible reaction mechanism during modified Tebbe-Claisen leading to observed products. 
At this point, this line of research was terminated due to less than adequate yields and a lack of control over the stereoselectivity of the products formed in this reaction.

Subsequent conversations with Fairbanks revealed that unless the titanocene oxide was rigorously excluded from the reaction, then problems arose during the Claisen rearrangement leading to a mixture of products. The residual titanocene could be catalysing the $S_{N} 1$ mechanism described above by lowering the energy required for activation of the leaving group. The oxonium intermediate formed is then intercepted by the free nucleophilic leaving group, leading to the observed results. Therefore it remains possible that this approach could provide the desired stereoselectivity.

\subsection{Summary}

The effects of using D-galactal and a different O-6 protecting group under Hoberg's ring expansion conditions were investigated. The selected silyl protecting group was found to be unsuitable under the conditions used. Several alternative expansion methods were also investigated. These were found to be unpredictable in terms of both products and stereoselectivities and were also low yielding. No firm conclusions could be reached on the effects of the galactal on the ring expansion stereoselectivity.

The use of an intermolecular nucleophile was also examined. While the regioselectivity of the ring expansion was excellent, no stereoselectivity could be induced and the yields were uninspiring. 


\section{Chapter 3: Synthesis and ring expansions of cyclopropanated galactal and glucal derivatives}

The work described in chapter two provided robust methodology for the synthesis of protected glycals and their subsequent cyclopropanation. However, further development of the methodology was required to achieve useful yields and stereoselectivities. Results presented in Section 2.4 and ealier work by Hoberg ${ }^{70}$ both pointed to the value of retaining the di-tert-butylsilyl protecting group, given its proven stability under the required reaction conditions. The work in this chapter explores the Ferrier rearrangement with a variety of nucleophiles. It was thought that the syn $\mathrm{C}-4$, C-5 stereochemistry of the galactal system would lead to better stereocontrol of the ring expansion than was evident in the corresponding anti arrangement in the previously explored glucals. It was proposed that the relative stereochemistry in the galactal system would force the silyl protecting group into a position where it would block the $\beta$-face and direct the nucleophile to attack from the $\alpha$ face.

Molecular mechanics modelling was used to test the soundness of these propositions. The structures of the cyclopropanated di-tert-butylsilyl derivatives of glucal (Figure 3.1) and galactal (Figure 3.2) were modelled. The molecular modelling indicates that the glucal system is held in a planar arrangement due to the di-tert-butylsilyl protecting group which creates a bicyclic ring system with a trans-fused ring junction (Figure 3.1). 


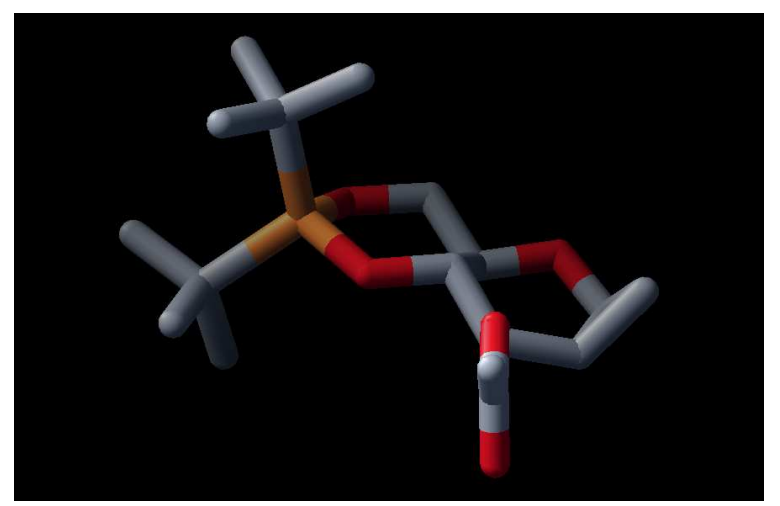

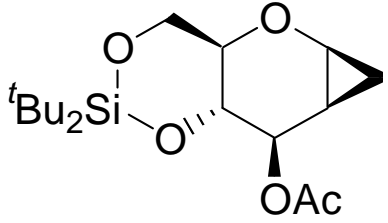

1.61

Figure 3.1. Cyclopropanated glucal system 1.61.

In the galactal system the di-tert-butylsilyl protecting group combined with the stereochemistry at C-4 creates a bicyclic ring system with a cis-fused ring junction. This forces the molecule to adopt a shape where the $\beta$ face is sterically hindered (Figure 3.2). This proposed structure is supported by the ${ }^{1} \mathrm{H}$ NMR of the cyclopropanated galactal 3.4. The cyclopropane protons of this compound are in notably different environments unlike those observed in glucal $\mathbf{1 . 6 1}$ which indicate that one of the protons is in a more congested environment.

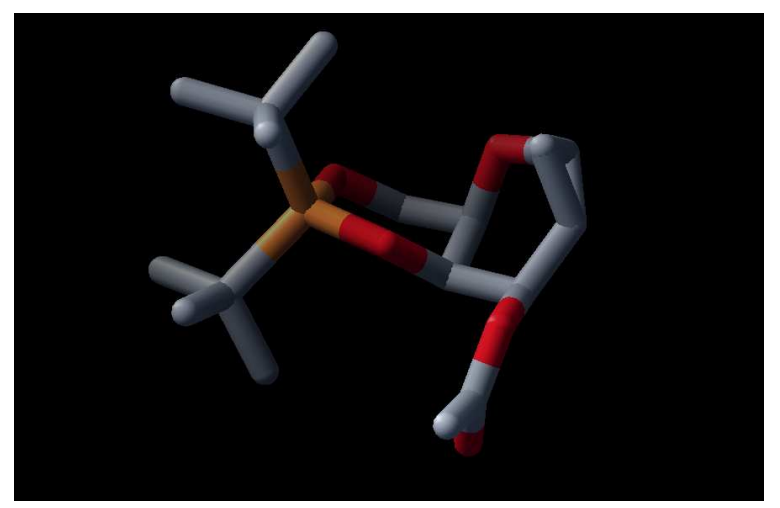

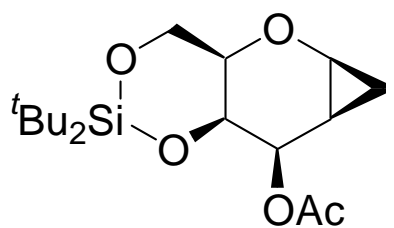

3.4

Figure 3.2. Cyclopropanated galactal system 3.4.

Possible structures for the corresponding oxonium ions were also modelled. The glucal-derived oxonium intermediate adopts a planar shape with a slightly convex $\beta$ face (Figure 3.3). This explains the results observed by Hoberg with this system where 
$\beta$-substitution predominated over $\alpha^{70}$ The shape of the intermediate overrides the normal direction of the Ferrier rearrangement resulting in the opposite stereochemistry to that expected.
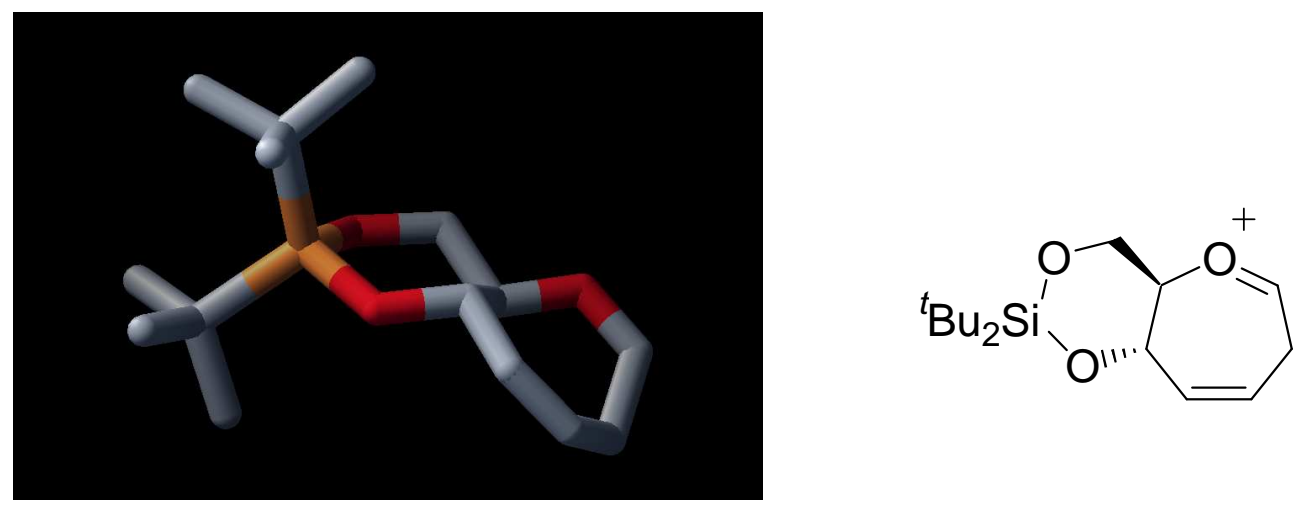

Figure 3.3. Glucal derived oxonium intermediate.

In contrast, the molecular modelling of the galactal oxonium intermediate suggests that the cis-fused bicyclic ring has a convex $\alpha$ face (figure 3.4). The $\beta$ face is thus hindered and the nucleophile should preferentially attack from the $\alpha$ face.
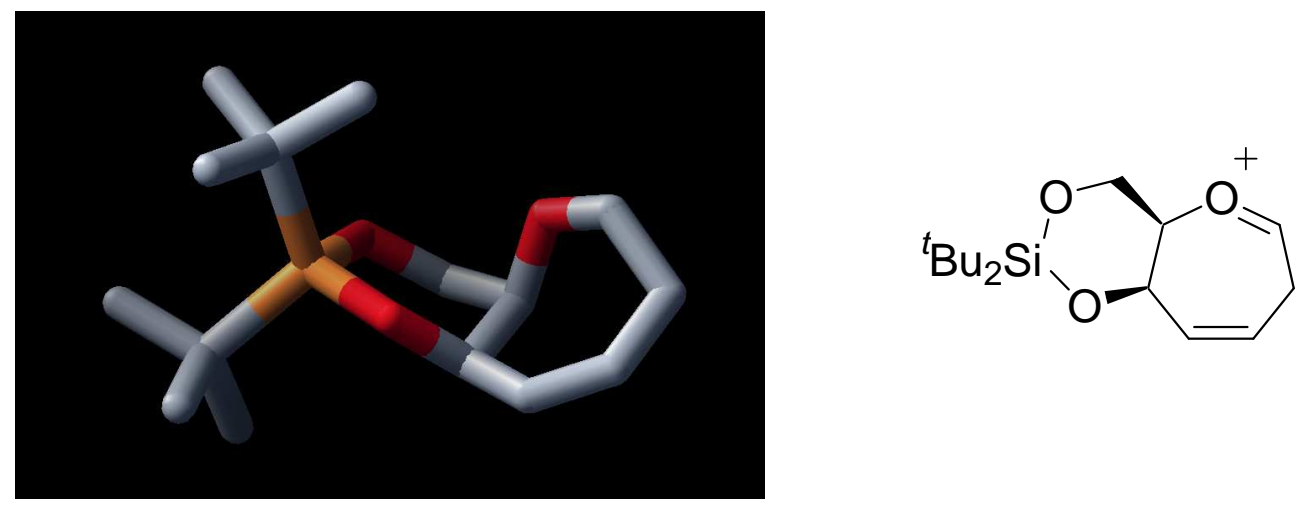

Figure 3.4. Galactal-derived oxonium intermediate.

The molecular modelling studies suggested that the strategy of using the di-tertbutylsilyl protecting group in combination with galactal in order to improve the stereoselectivities was sound. 


\subsection{Synthesis and reactions of cyclopropanated di-tert-butylsilyl galactal}

Synthesis of the desired cyclopropanated galactal 3.4 proceeded smoothly over four steps from galactal using the same strategy as applied by Hoberg in the synthesis of the corresponding cyclopropanated glucal (Scheme 3.1). ${ }^{70}$

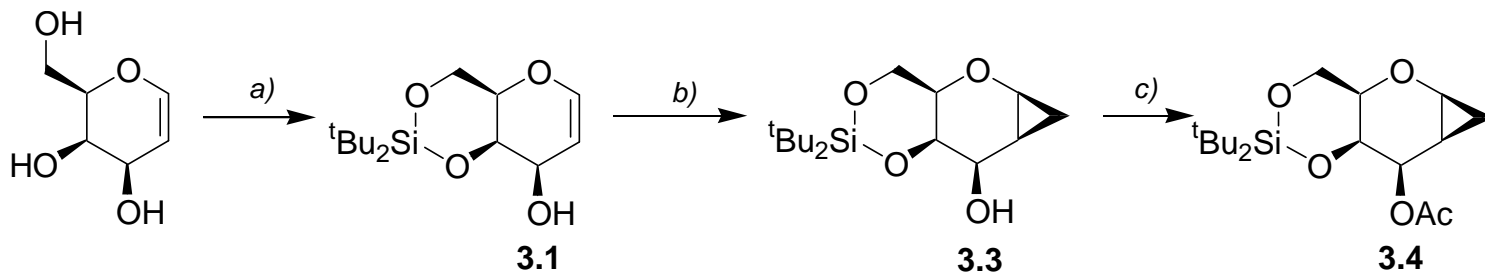

a) ${ }^{t} \mathrm{Bu}_{2} \mathrm{Si}(\mathrm{OTf})_{2}$, py, DMF, 65\%. b) $\mathrm{Et}_{2} \mathrm{Zn}, \mathrm{CH}_{2} \mathrm{I}_{2}, \mathrm{Et}_{2} \mathrm{O} . c$ ) $\mathrm{Ac}_{2} \mathrm{O}$, py, DMAP, 65\% over 2 steps.

Scheme 3.1. Reaction scheme for the formation of cyclopropane 3.4.

Silylation of galactal with di-tert-butylsilyl ditriflate in DMF gave glycal 3.1 in $65 \%$ yield along with the 3,4 di-tert-butylsilyl galactal 3.2 in 20\% yield (Figure 3.5). This compound was consistently present as a by-product of this reaction.

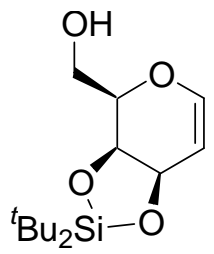

3.2

Figure 3.5. By-product 3.2 from formation of silyl ether 3.1.

Cyclopropanation of galactal 3.1 applying the Furukawa modification of the SimmonsSmith cyclopropanation ${ }^{39}$ using $\mathrm{Et}_{2} \mathrm{Zn}$ and $\mathrm{CH}_{2} \mathrm{I}_{2}$ followed by acetylation gave cyclopropane 3.4 in $65 \%$ yield over the two steps. 


\subsubsection{Ring expansions of cyclopropane 3.4}

Ring expansion with TMSOTf and a variety of TMS-substituted nucleophiles were explored yielding the corresponding oxepines (Scheme 3.2).

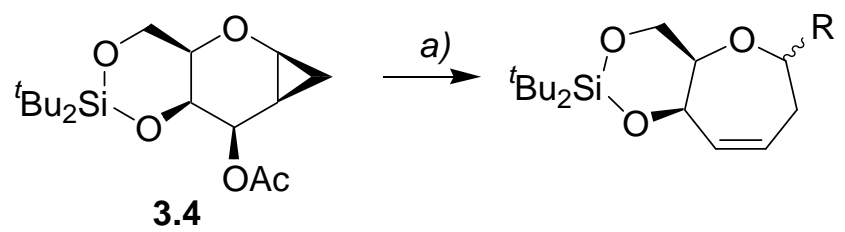

a) TMSR, TMSOTf, MeCN.

Scheme 3.2. General method for the expansion of cyclopropane 3.4.

The nucleophiles chosen for this initial series of reactions were $\mathrm{TMSN}_{3}, \mathrm{TMSSPh}$, TMSallyl, trimethyl(propargyl)silane (TMSpropargyl), and TMSOallyl. The results of these reactions are summarised in Table 3.1.

In the initial ring expansion in this series, cyclopropane 3.4 was reacted with $\mathrm{TMSN}_{3}$ in $\mathrm{MeCN}$ at $0{ }^{\circ} \mathrm{C}$ for four hours, forming oxepine 3.5 in a $69 \%$ yield with a $2.5: 1$ mix of epimers as determined by GC analysis (entry one, Table 3.1). It proved possible to separate the epimers by flash chromatography on silica gel. A complete NMR analysis of the structure of both epimers is provided in appendix A. NOE data for the minor isomer showed enhancements from $\mathrm{H}-1$ to $\mathrm{H}-2 \alpha$ and $\mathrm{H}-6$, assigning the azide group at C-1 as the $\beta$ epimer (Figure 3.6). This then assigns the major product as the $\alpha$ epimer. 
Table 3.1. Results of initial ring expansions reactions of $\mathbf{3 . 4}$.

\begin{tabular}{|c|c|c|c|c|}
\hline Entry & Nucleophile & Product & Yield & $\alpha: \beta$ ratio $^{a}$ \\
\hline 1 & $\mathrm{TMSN}_{3}$ & & $69 \%$ & $2.5: 1$ \\
\hline 2 & TMSSPh & & $85 \%$ & $6: 1$ \\
\hline 3 & TMSallyl & & $91 \%$ & $80: 1$ \\
\hline 4 & TMSpropargyl & & $68 \%$ & $7: 1$ \\
\hline 5 & TMSOallyl & - & $0 \%$ & - \\
\hline
\end{tabular}

a: determined by GC

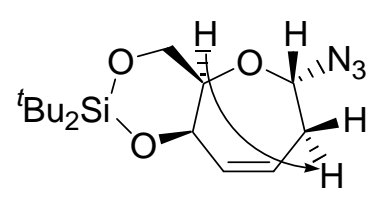

major isomer 3.5a

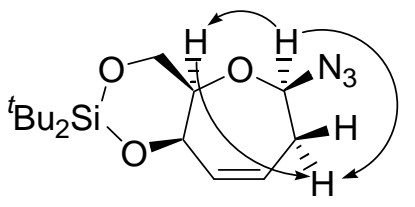

minor isomer $\mathbf{3 . 5 b}$

Figure 3.6. Selected NOE enhancements which were used to assign the stereochemistry of the major 3.5a and minor 3.5b isomers.

The next nucleophile in the series was TMSSPh. Reaction with cyclopropane $\mathbf{3 . 4}$ in MeCN with TMSOTf at ambient temperature and stirring until complete by TLC (five hours) gave oxepine $\mathbf{3 . 6}$ in $85 \%$ yield (entry two, Table 3.1). GC analysis indicated an improvement in stereoselectivity to $6: 1$. While the stereochemistry of the addidtion 
could not be determined, based on the previous result the major epimer was assumed to be $\alpha$.

The first of the carbon nucleophiles in the series was TMSallyl. Using the same conditions as used for the $\mathrm{TMSN}_{3}$ reaction but initiating the reaction at $-20{ }^{\circ} \mathrm{C}$ and allowing to warm to $0{ }^{\circ} \mathrm{C}$ until complete by TLC (one hour) gave oxepine 3.7 in a $91 \%$ yield (entry three, Table 3.1). GC analysis revealed a greater than 10 -fold increase in selectivity to $80: 1 .{ }^{1} \mathrm{H}$ NMR could not be used to determine the major epimer and so precedence from the other reactions was used to assign the major product as the $\alpha$ epimer.

The second carbon nucleophile was TMSpropargyl. Reaction with cyclopropane $\mathbf{3 . 4}$ at $0{ }^{\circ} \mathrm{C}$ until the reaction was complete by TLC (one hour) gave oxepine 3.8 in a $68 \%$ yield (entry four, Table 3.1). GC analysis revealed a decrease in selectivity to 7:1. An NOE enhancement was observed from the ring junction oxymethine of the major product at $\delta_{\mathrm{H}} 3.95$ to the proton on the ketene at $\delta_{\mathrm{H}} 5.26$ allowing the major epimer to be assigned as $\alpha$ (Figure 3.7).

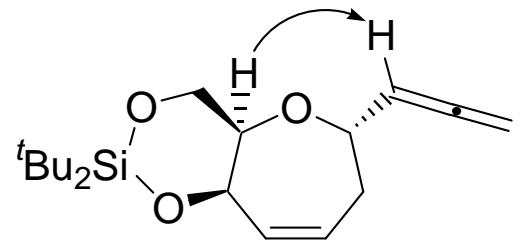

\section{8}

Figure 3.7. NOE enhancement used to assign the stereochemistry of the major epimer of $\mathbf{3 . 8}$.

Last in the initial series of reactions was that with an oxygen nucleophile, TMSOallyl (entry five, Table 3.1). The equivalent reaction was unsuccessful in Hoberg's expansions of $\mathbf{1 . 6 1}^{70}$ and that also proved to be the case here with no starting materials or products isolated. 
These results compare quite favourably with those obtained with the cyclopropanated glucal 1.61. The significant difference is that the galactal system ring expansions generate excesses of the $\alpha$-substituted oxepines. This selectivity bias is also observed in Ferrier reactions on uncyclopropanated glycals and will be discussed in Section 3.3.

The failure of the TMSOallyl nucleophile can be rationalized by examining the mechanism of formation of the substituted oxepines. The reaction proceeds in two stages. The first is the formation of the oxonium intermediate. This occurs with a concerted opening of the cyclopropane ring coupled with the departure of the leaving group (Figure 3.8).

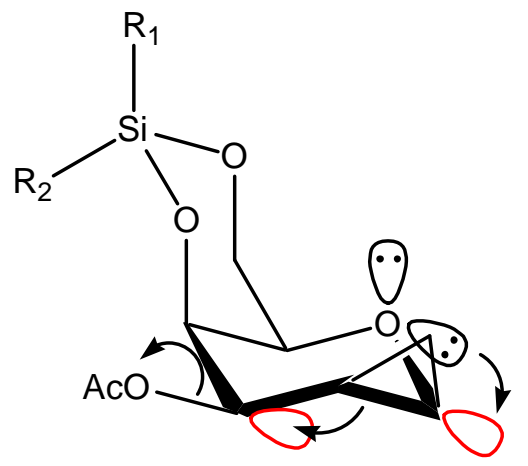

Figure 3.8. Formation of oxonium intermediate with $\sigma^{*}$ orbitals shown in red.

The oxonium ion is then intercepted by the TMS nucleophile to generate the oxepine product. The final outcome of the reaction depends on both the $\mathrm{K}_{1}$ and $\mathrm{K}_{2}$ equilibria as shown in Scheme 3.3.

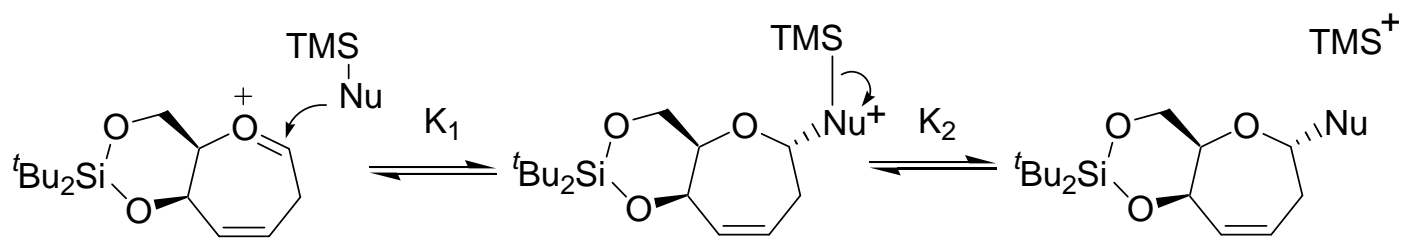

Scheme 3.3. Equilibria involved in the interception of the oxonium intermediate with a TMS nucleophile. 
The equilibrium constant $\mathrm{K}_{1}$ depends on the energy required to form the bond between C-1 and the incoming nucleophile. The equilibrium constant $\mathrm{K}_{2}$ depends on the relative energies of the breaking of the newly formed bond and the breaking of the NucleophileSi bond. Provided $\mathrm{K}_{1}$ is not insignificantly small then the formation of the final oxepine product is determined by the value of $\mathrm{K}_{2}$. Thus the outcome of these reactions could be interpreted as informative on the relative values of these two equilibrium constants. For example, for the first four entries in table $3.1, \mathrm{~K}_{2}$ is large enough so that the reaction proceeds to generate the desired oxepine in good yields. However when the nucleophile is TMSOallyl (entry five, table 3.1) the energy required to break the bond between the Nucleophile and C-1 is less than that required to break the Nu-TMS bond. This would make $\mathrm{K}_{2}$ less than one. With $\mathrm{K}_{1}$ likely to be much less than one, the outcome will be similar to the situation where no nucleophile is present. In this case no oxepine products are isolated and the galactal starting material decomposes to unidentifiable polymeric compounds. It should be noted here that the ring expansion reaction with only TMS triflate (no nucleophile) present in the reaction also decomposes the starting cyclopropane 3.4 to unidentifiable polymeric compounds.

\subsubsection{Investigation of the role of sterics in the ring expansion of cyclopropane 3.4}

To investigate the role that sterics could play in the reaction, two carbon nucleophiles with similar structures were chosen for trials. These were the 1-(trimethylsilyl)oxy-1ethoxyethene nucleophile 3.9 and its 2-methylpropene analogue 3.10 (Figure 3.9). 
<smiles>C=C(O[SiH3])OCC</smiles>

3.9<smiles>CCOC(OS)=C(C)C</smiles>

3.10

Figure 3.9. TMS nucleophiles to investigate steric constraints of the reaction.

The results for these reactions are summarized in Table 3.2.

Table 3.2. Ring expansions of cyclopropane 3.4 with nucleophiles 3.9 and $\mathbf{3 . 1 0}$.

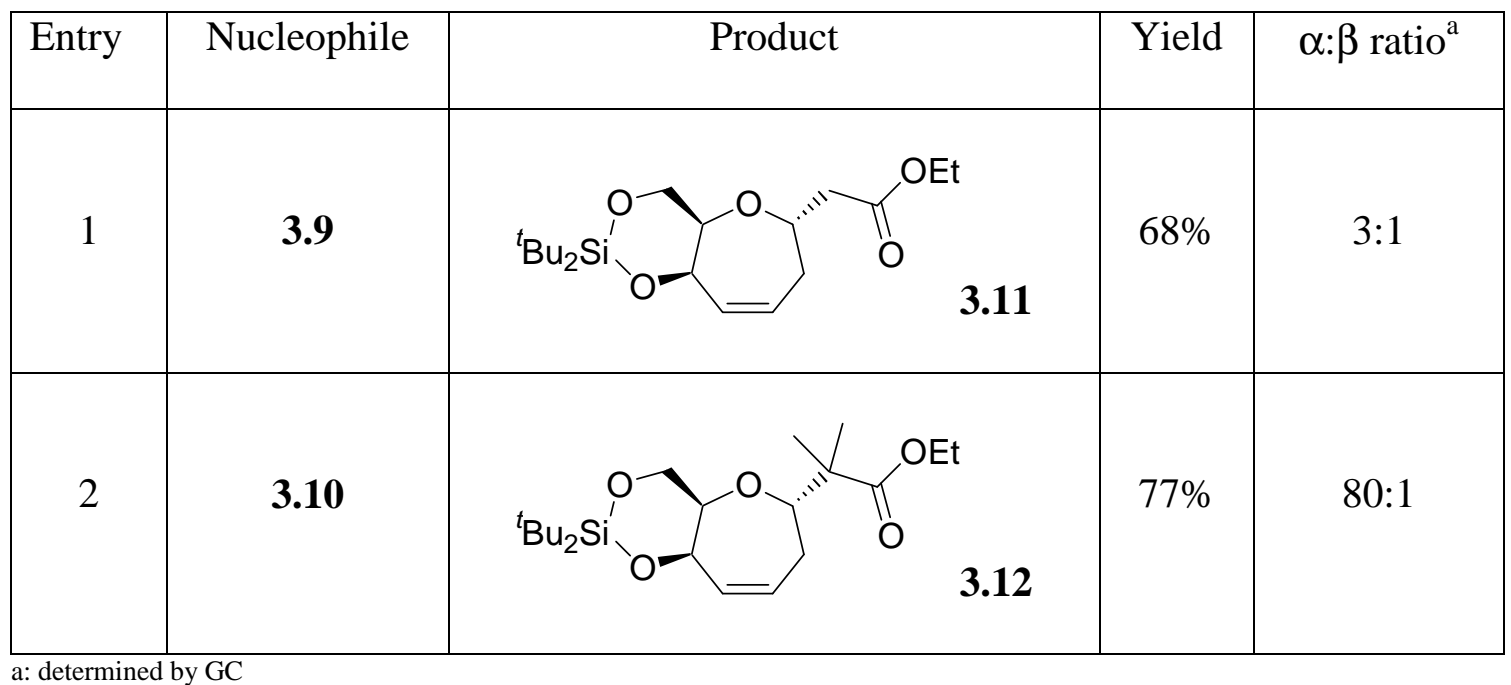

Reacting cyclopropane 3.4 with 3.9 in $\mathrm{MeCN}$ as before but starting at $0^{\circ} \mathrm{C}$ and warming to room temperature until complete by TLC (four hours) gave oxepine $\mathbf{3 . 1 1}$ in a $68 \%$ yield (entry one, Table 3.2). GC analysis revealed that the selectivity was only 3:1 with the major epimer assumed to be the $\alpha$ from previous work.

The equivalent reaction with 3.10, stirring overnight at ambient temperature until the reaction was complete by TLC resulted in oxepine $\mathbf{3 . 1 2}$ in a $77 \%$ yield (entry two, Table 3.2). GC analysis indicated that the selectivity had improved by an order of magnitude, to $80: 1$.

Again, it was possible to assign the major epimer by examination of the NOE data. In oxepine 3.12 an NOE enhancement was observed from the H-6 oxymethine at $\delta_{\mathrm{H}} 4.00$ 
to the $\mathrm{H}-2 \alpha$ methylene proton at $\delta_{\mathrm{H}} 2.51$ which is in an axial position on the $\alpha$ face. The H-2 $\alpha$ methylene proton then demonstrates an NOE enhancement to the gemdimethyl system at $\delta_{\mathrm{H}} 1.16$ and 1.11, assigning the major epimer as $\alpha$ (Figure 3.10).

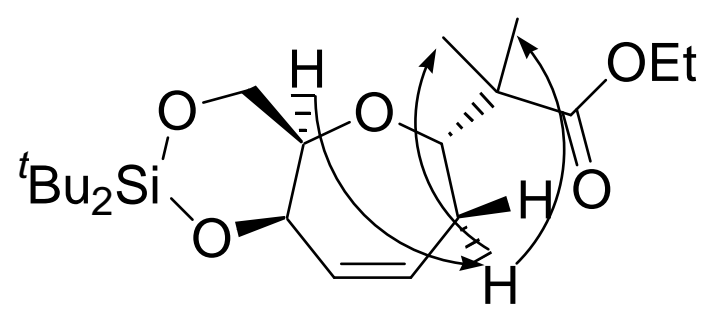

Figure 3.10. NOE enhancements assigning the structure of the major epimer 3.12.

\subsection{Ring expansions of cyclopropanated TBDPS protected glucal}

Following the favourable results from section 3.1, the next logical step was to reinvestigate the possibility that the results could be replicated with a bulky silyl group that was mono-tethered to the galactal frame. This revisits the line of investigation in chapter two with several important differences based on experience gained with cyclopropanated galactal 3.4. Firstly, the tert-butyldiphenylsilyl protecting group was chosen as it had the desired bulk to direct the nucleophilic attack. It was also more stable under acidic conditions than the previously investigated TIPS group. Secondly, the glucal frame was chosen in order to avoid the problems acetylating the diol $\mathbf{2 . 3}$ described in Section 2.1. This should prevent the generation of triflic acid from the reaction of TMSOTf and the free alcohol leading to the observed de-silylation and decomposition during the ring expansion reactions as previously described. Possible drawbacks are that the monodentate protecting group would not hold the cyclopropanated glucal ring in a rigid conformation and that the bulky protecting group would adopt a conformation well away from the ring due to the lack of structural constraints. The synthetic approach is summarised in Scheme 3.4. 

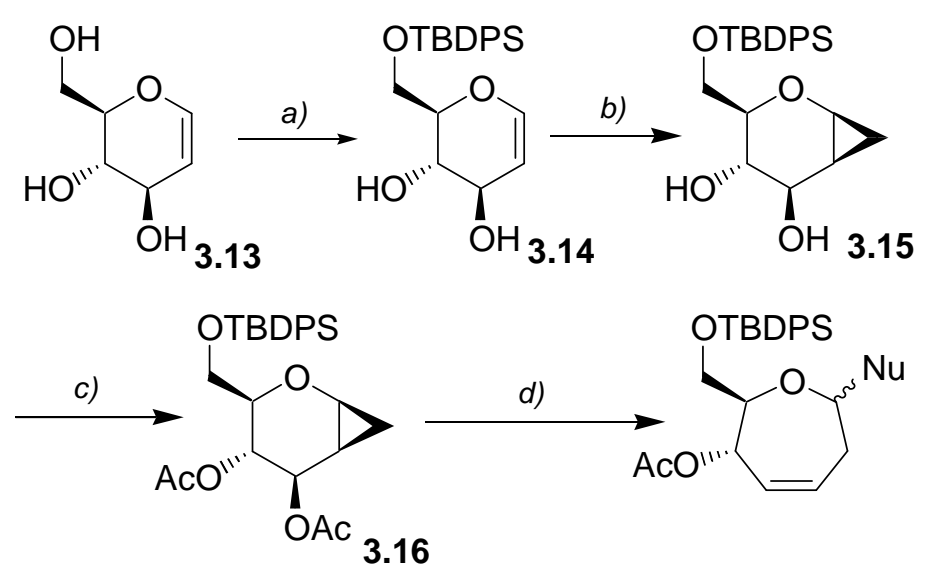

a) TBDPSCl, Imidazole, THF, RT, 65\%. b) $\mathrm{Et}_{2} \mathrm{Zn}, \mathrm{CH}_{2} \mathrm{I}_{2}, \mathrm{Et}_{2} \mathrm{O}$ reflux, 77\%.c) $\mathrm{Ac}_{2} \mathrm{O}, \mathrm{Py}$, DMAP, $\mathrm{CH}_{2} \mathrm{Cl}_{2}, \mathrm{RT}, 96 \%$. d) 50 mol\% TMSOTf, TMSNu, MeCN, 10-70\% depending on $\mathrm{Nu}$

Scheme 3.4. Formation of $\mathbf{3 . 1 6}$ and its ring expansion.

Synthesis of cyclopropane $\mathbf{3 . 1 6}$ proceeded smoothly over three steps. Silylation of the galactal 3.13 with TBDPSCl and imidazole in THF formed silylether $\mathbf{3 . 1 4}$ in a $65 \%$ yield. Simmons-Smith cyclopropanation as before gave cyclopropane $\mathbf{3 . 1 5}$ in $77 \%$ yield with $>100: 1$ stereoselectivity for the expected $\beta$ isomer. In contrast to $\mathbf{2 . 3}$, diol 3.15 was easily acetylated in $96 \%$ yield to give the bis-acetylated cyclopropane $\mathbf{3 . 1 6}$ in $48 \%$ overall yield.

The same nucleophiles chosen for the ring expansions of cyclopropane $\mathbf{3 . 5}$ were used in this series of expansions. The results for this series are summarised in Table 3.3.

Reaction of cyclopropane 3.16 with $\mathrm{TMSN}_{3}$ in $\mathrm{MeCN}$ at $0{ }^{\circ} \mathrm{C}$ for 30 minutes followed by warming to ambient temperature until complete by TLC (one hour) gave oxepine 3.17 in a $62 \%$ yield as a 2.5:1 mix of epimers by GC (entry 1, Table 3.3). This result indicated that the TBDPS protecting group was stable under the reaction conditions and the results were also remarkably similar in terms of yield and selectivity to those observed with oxepine $\mathbf{3 . 5}$. 
Table 3.3. Results of ring expansions of cyclopropane 3.16.

\begin{tabular}{|c|c|c|c|c|}
\hline Entry & Nucleophile & product & yield & $\alpha: \beta$ ratio \\
\hline 1 & $\mathrm{TMSN}_{3}$ & OTBDPS & $62 \%$ & 2.5:1 \\
\hline 2 & TMSSPh & OTBDPS & $45 \%$ & $3: 1$ \\
\hline 3 & TMSallyl & Complex mixture including $\mathbf{4 . 3}$ & $0 \%$ & - \\
\hline 4 & TMSpropargyl & OTBDPS & $8 \%$ & $7: 1$ \\
\hline 5 & TMSOallyl & - & $0 \%$ & - \\
\hline
\end{tabular}

The next expansion was with TMSSPh. Reaction under similar conditions as used with this nucleophile and $\mathbf{3 . 4}$, but stirring at ambient temperature until the reaction was complete by TLC (five hours) gave oxepine $\mathbf{3 . 1 8}$ in a $45 \%$ yield as a 3:1 mixture of epimers by GC (entry two, table 3.3). This represented a decrease in stereoselectivity and also in yield compared to oxepine 3.6.

Reactions in the series continued with TMSallyl. Reaction with this nucleophile at $-40^{\circ} \mathrm{C}$ and subsequent warming to ambient temperature until no starting material was left by TLC (four hours) gave a complex mixture of products. Separation revealed an unexpected product that will be further discussed in chapter four. 
Reaction with TMS propargyl gave the expected oxepine 3.19 as the $\alpha$ epimer exclusively by ${ }^{1} \mathrm{H}$ NMR. However, it was only isolated in an $8 \%$ yield (entry four, table 3.3). As no other products could be isolated from this reaction, no further information could be obtained as to the fate of the rest of the starting material.

TMSOallyl was used to complete the set of initial nucleophiles investigated. As before no products could be isolated nor starting material recovered from the reaction (entry five, table 3.3). It is proposed that the product of this reaction suffers a similar fate to that of the cyclopropanated galactal under these conditions.

From this set of results we can see that with the exception of entry one, the yields are inferior to those obtained with the galactal 3.4. From this it is possible to surmise that while the TBDPS protecting group is more stable than the TIPS group under the ring expansion conditions, it still undergoes deprotection to some extent. The yields appear to decrease with increasing reaction time and increasing initial temperature of the addition. The higher yield observed in the $\mathrm{TMSN}_{3}$ example is most likely a function of the short reaction time which means deprotection of the TBDPS group has not yet become a major factor. For several of the nucleophiles, however, no appreciable reaction occurred at lower temperatures or with shorter reaction times.

\subsubsection{Investigation of the role of sterics in the ring expansion of cyclopropane 3.16}

As in section 3.1.2 the two carbon nucleophiles $\mathbf{3 . 9}$ and $\mathbf{3 . 1 0}$ were used to examine the effect of nucleophile bulk on the selectivity of the ring expansion. In both of these reactions the expected oxepines were not observed in the products. The characterisation of the isolated products from these reactions will be discussed in section 4.2. 


\subsection{Effect of the leaving group stereochemistry on the ring expansion}

Previous investigations by Danishefsky ${ }^{22}$ have shown that the stereoselectivity of the Ferrier rearrangement is reduced when the leaving group is in an axial conformation (the allal ring system) compared to when the leaving group is equatorial (the glucal or galactal ring system). ${ }^{22}$ This experiment was replicated in the $3 R$ cyclopropanated glucal system $\mathbf{1 . 6 1}$ by inverting the stereochemistry of the leaving group at C-3 to give the corresponding $2 S$ cyclopropane $\mathbf{3 . 2 2}$ (Scheme 3.5).

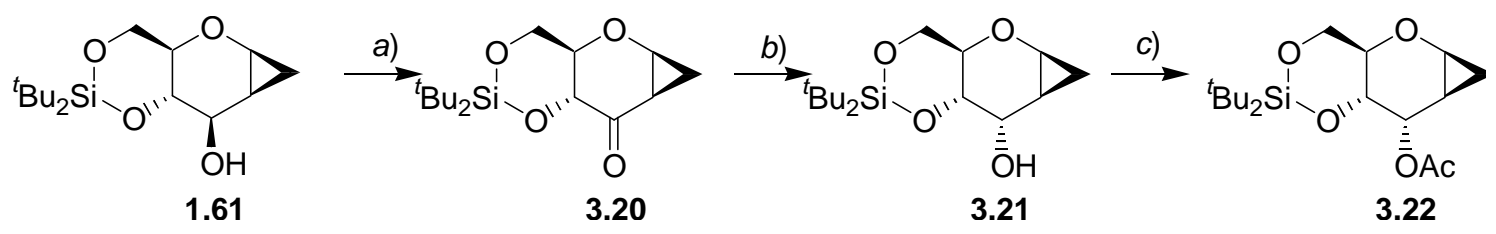

a) Oxalyl chloride, DMSO, TEA, $\mathrm{CH}_{2} \mathrm{Cl}_{2}, 90 \%$. b) $\mathrm{NaBH}_{4}, \mathrm{EtOH}, 24 \%$ c) $\mathrm{Ac}_{2} \mathrm{O}, \mathrm{Py}, 89 \%$.

Scheme 3.5. Formation of di-tert-butyl-silyl allal cyclopropane.

Oxidation of cyclopropane 1.61 using standard Swern conditions gave ketone 3.20 in 90\% yield. Ketone $\mathbf{3 . 2 0}$ was then reduced with $\mathrm{NaBH}_{4}$ to give a mixture of the glucal and allal cyclopropanes. The desired cyclopropanated allal $\mathbf{3 . 2 1}$ was isolated in $24 \%$ yield along with a 50\% return of glucal 1.61. Cyclopropane 3.21 was then acetylated to give the desired cyclopropane $\mathbf{3 . 2 2}$ in $89 \%$ yield. This substrate was then subjected to the standard ring opening conditions of TMSOTf in MeCN using TMSallyl as the nucleophile. This reaction was not complete by TLC until 48 hours had elapsed. The corresponding reaction with the glucal system was complete in two hours (Scheme 3.6). ${ }^{70}$ The yields and mixture of epimers were very similar in both cases $(92 \%, 1.1$ ds). 


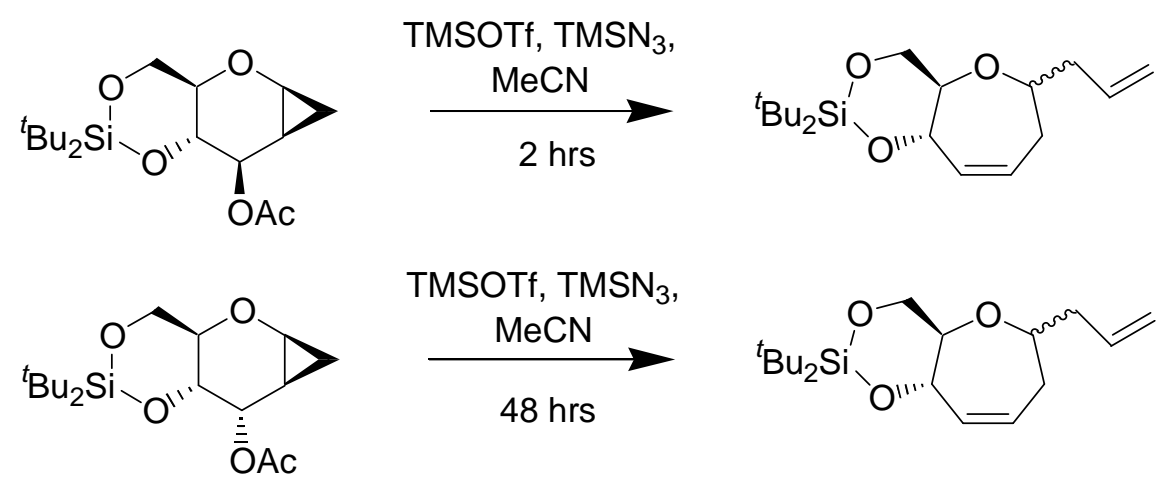

Scheme 3.6. Effect of the stereochemistry of the leaving group on the rate of reaction.

Analysis of the structures of the compounds $\mathbf{1 . 6 1}$ and $\mathbf{3 . 2 2}$ indicates that in the glucal 1.61 the departure of the equatorial leaving group is aided by the favourable syn periplanar geometry of the $\sigma^{*}$ orbital associated with the leaving group bond with respect to the cleaved cyclopropyl $\sigma$ bond. Electrons from the breaking bond are pushed into the $\mathrm{C}-\mathrm{O} \sigma^{*}$ orbital at $\mathrm{C}-3$ forming a $\mathrm{C}-\mathrm{C}$ double bond. This is assisted by the overlap of the ring oxygen lone pair with the $\sigma^{*}$ orbital at $\mathrm{C}-1$, leading to the formation of the $\pi$ bond of the oxonium intermediate (Figure 3.11).

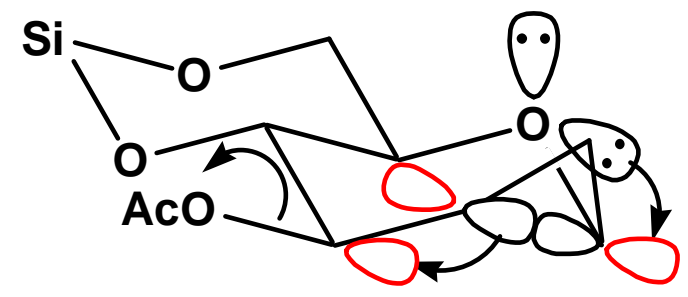

Figure 3.11. Mechanism for the formation of the oxonium intermediate from cyclopropanated glucal $\mathbf{1 . 6 1}\left(\sigma^{*}\right.$ orbitals shown in red).

In the allal 3.22, the $\sigma^{*}$ orbital of the axial leaving group is not aligned in a synperiplanar relationship with the breaking cyclopropane bond. As a result, the formation of the oxonium intermediate is kinetically disfavoured, experimentally 20 times longer. This is illustrated in Figure 3.12. 


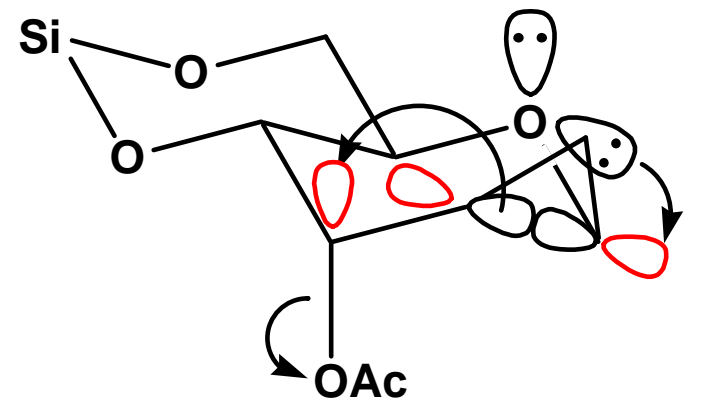

Figure 3.12. Mechanism for the formation of the oxonium intermediate from cyclopropanated allal 3.22.

\subsection{Comparison with glycal glycoside rearrangements}

Finally the results obtained by the ring expansions of cyclopropanated glycals 3.4 and

3.16 were compared to results for Ferrier rearrangments from the literature with glycals lacking a cyclopropane group. These are summerised in Table 3.4.

Table 3.4. Summary of ring expansions of cyclopropanes 3.4 and $\mathbf{3 . 1 6}$ verses glycal rearrangements.

\begin{tabular}{|c|c|c|l|l|l|l|l|l|}
\hline Base sugar & \multicolumn{2}{|l|}{ Galactal 3.4 } & \multicolumn{2}{l|}{ Galactal lit. } & \multicolumn{2}{l|}{ Glucal 3.16 } & \multicolumn{2}{l|}{ Glucal lit. } \\
\hline Nucleophile & ratio & yield & ratio & yield & ratio & yield & ratio & yield \\
\hline TMSN $_{3}$ & $2.5: 1$ & $69 \%$ & $4: 1^{89}$ & $94 \%$ & $2.5: 1$ & $61 \%$ & $3: 1^{90}$ & $42 \%$ \\
\hline TMSSPh & $6: 1$ & $85 \%$ & $\alpha$ only $^{91}$ & $96 \%$ & $6: 1$ & $45 \%$ & $9: 1^{92}$ & $80 \%$ \\
\hline TMSallyl & $80: 1$ & $91 \%$ & $\alpha$ only $^{23}$ & 97 & $-^{\mathrm{a}}$ & $-^{\mathrm{a}}$ & $88: 1^{25}$ & $91 \%$ \\
\hline TMSpropargyl & $7: 1$ & $68 \%$ & $\alpha$ only $^{93}$ & $75 \%$ & $\alpha$ only $^{25}$ & $8 \%$ & $\alpha$ only $^{93}$ & $88 \%$ \\
\hline 3.9 & $3: 1$ & $68 \%$ & $\beta$ only $^{94}$ & $70 \%$ & $6: 1^{\mathrm{b}}$ & $81 \%$ & $1: 2.4^{94}$ & $81 \%$ \\
\hline 3.10 & $80: 1$ & $77 \%$ & $1: 4.3^{94}$ & $64 \%$ & $\alpha$ only $^{\mathrm{c}}$ & $67 \%$ & $1: 4^{94}$ & $67 \%$ \\
\hline
\end{tabular}

a) generates $\mathbf{4 . 3}$

All ratios are $\alpha: \beta$.

b) rearranges to give diene $\mathbf{4 . 1}$

c) rearranges to give diene $\mathbf{4 . 2}$ 
It should be noted that in most of the literature cases the reaction conditions were similar in terms of the use of a Lewis acid to effect the Ferrier rearrangement and are provided as comparative examples.

This table shows that the stereoselective outcomes of the ring expansions of cyclopropanes 3.4 and $\mathbf{3 . 1 6}$ are comparable with those obtained from Ferrier rearrangements of glycal systems. The yields are generally lower or equivalent.

An interesting result is that observed in the case of the reactions with nucleophiles $\mathbf{3 . 9}$ and 3.10. This generates good to excellent $\alpha$ selectivities in the ring expansion reactions of 3.4 and 3.16. However in the glycal Ferrier rearrangement the predominant product is the $\beta$ compound. This can be explained by looking at the two possible products in each of these reactions. In the ring expansions, the methylene group at C-2 increases the flexibility of the product oxepine. The substituent at C-1 is not forced into an axial orientation in either isomer and there are no destabilising interactions with other axial groups present in the ring. In the rearrangement of the pyranyl system, the $\alpha$ product is destabilised by the diaxial interaction between the gem-dimethyl group and the axial ring hydrogen (Figure 3.13). In the $\beta$ product where the gem-dimethyl is equatorial these interactions are not present, and the product formation is more favourable.

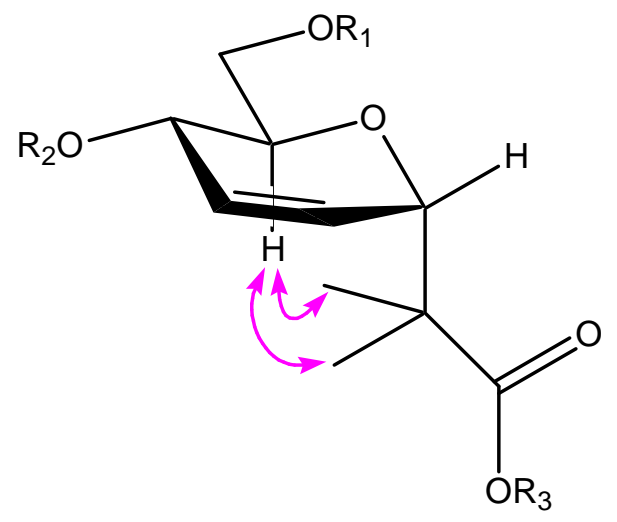

Figure 3.13. Diaxial interactions in Ferrier rearrangement of glycals and nucleophiles 3.9 or 3.10. 


\subsection{Summary}

Hoberg's work with the glucal system revealed increased $\beta$ selectivity of the ring expansion compared to the standard Ferrier rearrangement. ${ }^{70}$ The bicyclic trans-fused system forces the oxonium intermediate into a slightly convex form making attack from the $\beta$ face more facile. This is the opposite stereochemistry to that observed for the normal Ferrier rearrangement, which is predominantly $\alpha$. The bicyclic cis fused galactal system forces the oxonium intermediate into a bowl-like structure that makes nucleophilic attack more facile at the $\alpha$ face. This improves the stereoselectivity of the reaction. The yields for the oxepine formation remain high. The presence of the methylene in the oxepine ring gives the structure some measure of flexibility that is demonstrated by the reduced selectivities of some of the nucleophiles compared to the analogous pyranyl ring systems. The results from the monosilylated glucal system demonstrate that with no rigidity in the ring, a similar level of stereoselectivity to that of the hexose ring rearrangement is observed. The ring expansion results demonstrate that this extension of the Ferrier rearrangement is a synthetically useful stereoselective method for the formation of substituted seven membered rings. 


\section{Chapter 4: Unexpected rearrangements obtained from ring expansions and mechanistic rational}

In the course of the ring expansion reactions of the cyclopropanes 3.4 and 3.16 several unexpected results occurred (these are noted in Sections 3.1 and 3.2). In all cases it was possible to separate and purify the products. These compounds were identified with the assistance of 1D and 2D NMR, IR and in some cases ES HRMS. From the resulting structures it was then possible to postulate mechanisms to account for their formation.

\subsection{Unexpected reaction products from ring expansions of cyclopropanated glucal 3.16}

\subsubsection{Reaction with carbon nucleophiles 3.9 and 3.10}

Treatment of cyclopropane 3.16 with nucleophile 3.9 at $0{ }^{\circ} \mathrm{C}$ followed by warming to room temperature and stirring until the reaction was complete by TLC gave a 6:1 ratio of epimeric products. Analysis of the ${ }^{1} \mathrm{H}$ NMR spectrum revealed that neither the methylene signals normally observed at approximately $\delta_{\mathrm{H}} 2-2.5 \mathrm{ppm}$ nor the acetate methyl resonance were present. In addition, four new olefinic protons were identified in

the spectrum. The connectivity of the ${ }^{1} \mathrm{H}$ spin system for the major epimer was then constructed from a series of COSY correlations from the C-7 methylene $\left(\delta_{\mathrm{H}} 3.75, \delta_{\mathrm{C}}\right.$ $65.7)$ to an oxymethine $\left(\mathrm{C}-6: \delta_{\mathrm{H}} 4.26, \delta_{\mathrm{C}} 75.4\right)$ to a alkenic methine $\left(\mathrm{C}-5: \delta_{\mathrm{H}} 6.16, \delta_{\mathrm{C}}\right.$ 136.2) to a second alkenic methine $\left(\mathrm{C}-4: \delta_{\mathrm{H}} 6.05, \delta_{\mathrm{C}} 126.7\right)$, to a third alkenic methine $\left(\mathrm{C}-3: \delta_{\mathrm{H}} 6.04, \delta_{\mathrm{C}} 126.8\right)$ to a forth alkenic methine $\left(\mathrm{C}-2: \delta_{\mathrm{H}} 5.98, \delta_{\mathrm{C}} 137.0\right)$ to an 
oxymethine $\left(\mathrm{C}-1: \delta_{\mathrm{H}} 4.78, \delta_{\mathrm{C}} 72.7\right)$. This suggested that as well as the ring expansion there had been an elimination leading to the formation of a diene species (Scheme 4.1).

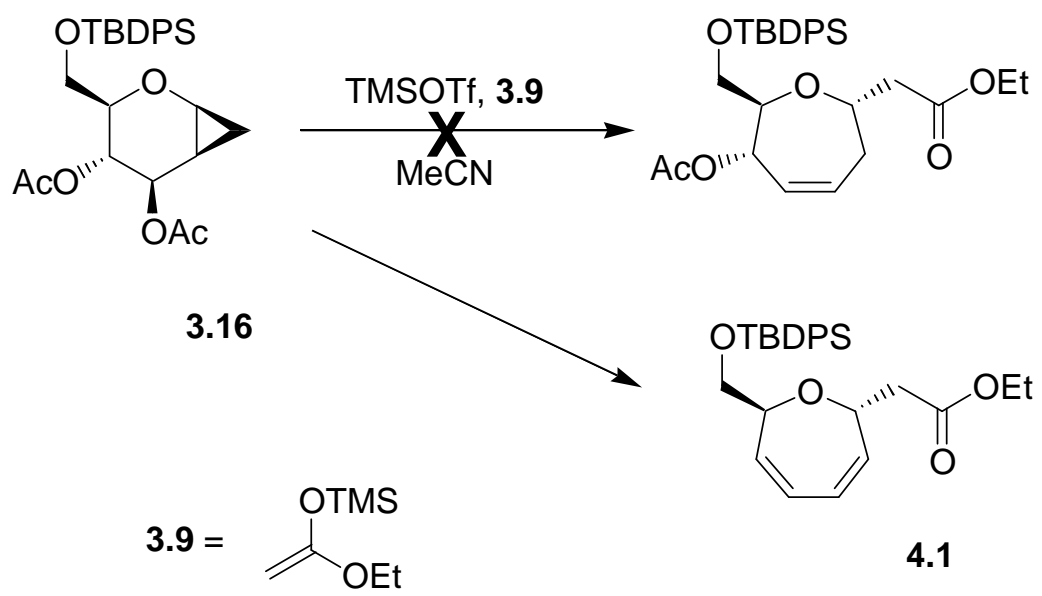

Scheme 4.1. Ring expansion of cyclopropane 3.16 with nucleophile 3.9.

A possible mechanism is suggested in Scheme 4.2. After the ring expansion the formation of the diene is facilitated by the presence of a second acetate group on the ring. The departure of this leaving group leads to the observed rearrangement.

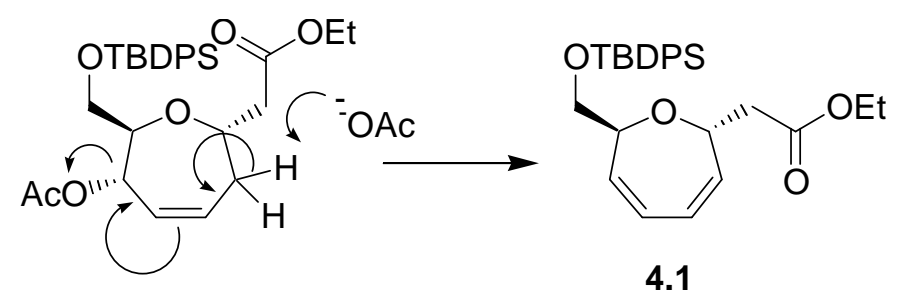

Scheme 4.2. Possible mechanism leading to the formation of oxepine 4.1.

An analogous result is seen in the reaction with cyclopropane $\mathbf{3 . 4}$ and the related dimethylated nucleophile 3.10. However, with the dimethylated species 4.2 (Figure 4.1) the yield was far lower which suggests that there were other competing mechanisms at work in the reaction. 


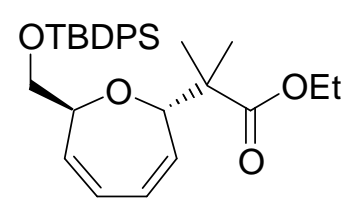

Figure 4.1. Product 4.2 from the ring expansion of cyclopropane 3.16 with nucleophile 3.10.

However, as no other compounds could be isolated from the products of this reaction, no further information could be obtained on the outcome of this reaction.

\subsubsection{Reaction of cyclopropane 3.16 with allyltrimethylsilane}

When the nucleophile TMSallyl was used in the ring expansion with cyclopropane 3.16 under standard conditions (5 eq TMSallyl, 0.5 eq TMSOTf in MeCN), the reaction resulted in a complex mixture of products. After repeated column chromatography on silica gel the major compound present was isolated. Initial analysis of the ${ }^{1} \mathrm{H}$ NMR spectrum suggested that the desired product had been formed. Closer examination of the 2-D NMR data revealed several inconsistencies. The HSQC-DEPT spectrum suggested the possibility of a mixture of two isomers in a 1:1 ratio. The ${ }^{13} \mathrm{C}$ spectra revealed that there were only three signals in the 60-80 ppm region, which is where oxymethines appear in ${ }^{13} \mathrm{C}$ NMR. This was evidence that there was only one compound present in the sample. The HSQC spectrum showed that there was a methylene at $\delta_{C}$ 66.2 and an oxymethine at $\delta_{\mathrm{C}} 69.9$. The third signal at $\delta_{\mathrm{C}} 81.5$ was a quaternary centre. A more detailed analysis of the compound's spectral data was then undertaken. An examination of the COSY data revealed the presence of three separate ${ }^{1} \mathrm{H}$ spin systems. The connectivity of the largest ${ }^{1} \mathrm{H}$ spin system was constructed from a series of COSY correlations from a terminal alkenic methylene $\left(\mathrm{C}-1: \delta_{\mathrm{H}} 5.0, \delta_{\mathrm{C}} 116.6\right)$ to an alkenic methine $\left(\mathrm{C}-2: \delta_{\mathrm{H}} 5.82, \delta_{\mathrm{C}} 135.6\right)$ to a methylene $\left(\mathrm{C}-3: \delta_{\mathrm{H}} 2.10\right.$ and $\left.2.25, \delta_{\mathrm{C}} 41.7\right)$ to an oxymethine $\left(\mathrm{C}-4: \delta_{\mathrm{H}} 4.13, \delta_{\mathrm{C}} 69.9\right)$ to a methylene $\left(\mathrm{C}-5: \delta_{\mathrm{H}} 2.05\right.$ and $\left.\delta_{\mathrm{H}} 2.28, \delta_{\mathrm{C}} 38.7\right)$ 
to an alkenic methine $\left(\mathrm{C}-6: \delta_{\mathrm{H}} 5.51, \delta_{\mathrm{C}} 129.6\right)$ to a second alkenic methine $\left(\mathrm{C}-7: \delta_{\mathrm{H}}\right.$ $\left.5.43, \delta_{\mathrm{C}} 124.5\right)$ to a methylene $\left(\mathrm{C}-8: \delta_{\mathrm{H}} 2.60\right.$ and $\left.2.35, \delta_{\mathrm{C}} 32.3\right)$ Interestingly this was where this substructure ended (Figure 4.2).

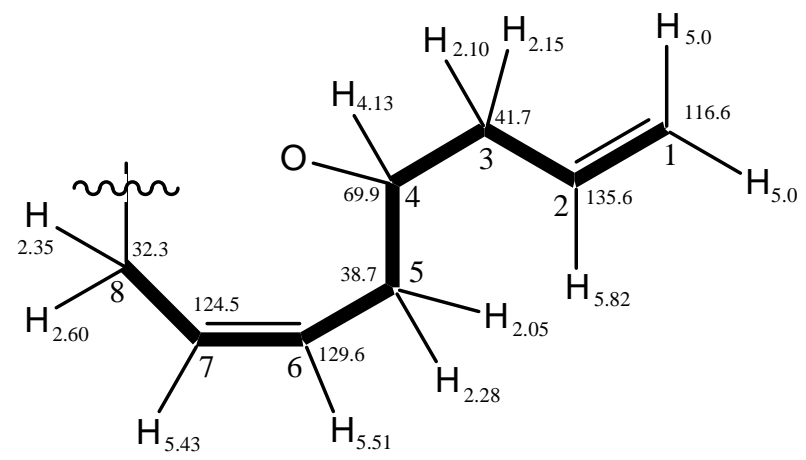

Figure 4.2. Large substructure from product of TMSallyl reaction with cyclopropane 3.16.

The connectivity of the second ${ }^{1} \mathrm{H}$ spin system was constructed from a series of COSY correlations from a terminal alkenic methylene $\left(\mathrm{C}-9: \delta_{\mathrm{H}} 5.10, \delta_{\mathrm{C}} 117.5\right)$ to an alkenic methine $\left(\mathrm{C}-10: \delta_{\mathrm{H}} 5.90, \delta_{\mathrm{C}} 134.4\right)$ to a methylene $\left(\mathrm{C}-11: \delta_{\mathrm{H}} 2.50\right.$ and $\left.2.40, \delta_{\mathrm{C}} 40.4\right)$. This was a second allyl group (Figure 4.3).

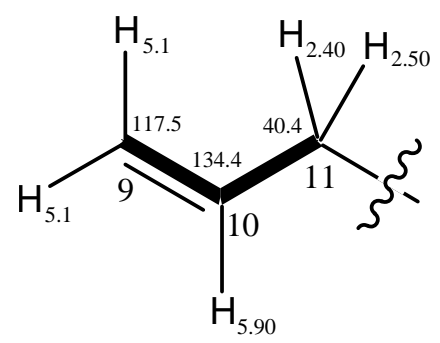

Figure 4.3. Small substructure from product of TMSallyl reaction with cyclopropane 3.16.

The other substructure was a lone oxymethylene $\left(\mathrm{C}-12: \delta_{\mathrm{H}} 3.55\right.$ and $\left.3.47, \delta_{\mathrm{C}} 66.2\right)$.

The connectivity between these three substructures was established by HSQC and HMBC correlations. Comparing the HSQC and ${ }^{13} \mathrm{C}$ spectra, a quaternary carbon was observed at $\delta_{\mathrm{C}} 81.5 \mathrm{ppm}$. This carbon exhibited HMBC correlations to two methylenes at $\mathrm{C}-11$ and $\mathrm{C}-12$ and also a weak correlation to the C-4 oxymethine. This then 
assigned the structure as the diallyl compound $\mathbf{4 . 3}$ as shown in Figure 4.4 with the identified substructures linked at the quaternary carbon numbered C-13.

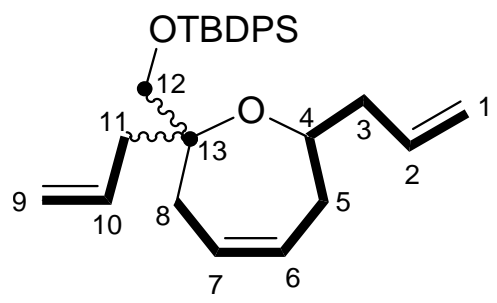

Figure 4.4. Structure of diallyl oxepine 4.3 numbered for the assignment in the text.

To gain more information on the possible mechanism directing this rearangement it was decided to repeat the reaction, but limit the amount of nucleophile present. This should lead to the generation of stable reaction intermediates, which could then be isolated and characterised. Repeating the reaction with 1.2 equivalents of TMSallyl using an identical work up followed by column chromatography on silica gel generated the products as shown in Figure 4.5. Interestingly, none of the diallyl 4.6 was isolated.

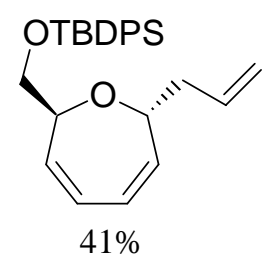

4.4

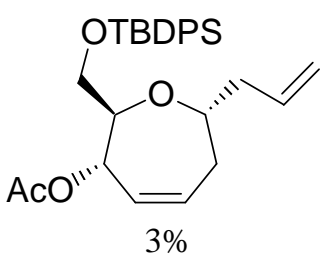

4.5

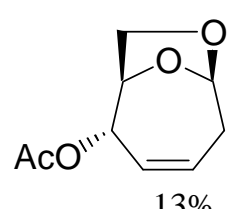

4.6
Starting material $23 \%$

3.16

Figure 4.5. Products from the reaction of TMSallyl with Cyclopropane 3.16.

The conjugated diene $\mathbf{4 . 4}$ was the prevalent species isolated, along with small amounts of the expected ring expanded oxepine 4.5. Also present was the bicyclic oxepine 4.6. This compound presumably results from deprotection of the silyl ether at C-7 prior to ring expansion as described in Chapter 2 (Scheme 2.2). This particular bicylic ring system had not been previously observed in the course of the ring expansion reactions discussed in this thesis although Hoberg and Bozell had previously reported them in the 
course of their work. ${ }^{40}$ However a similar bicyclic ether $\mathbf{2 . 7}$ had been observed as seen in Scheme 2.2.

It is possible to propose a mechanistic scheme to account for these results. This is illustrated in Scheme 4.3.

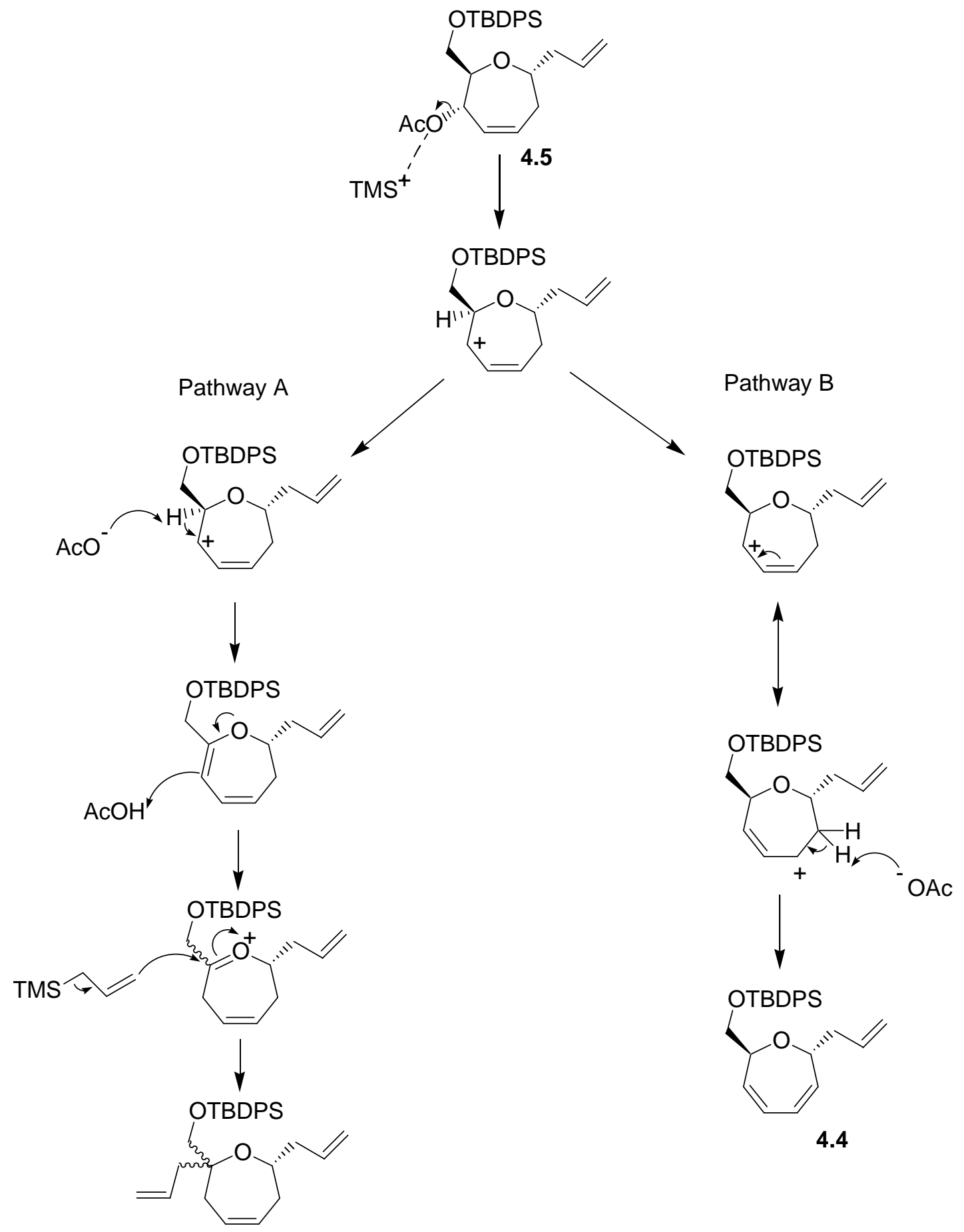

4.3

Scheme 4.3. Possible reaction mechanism to account for the formation of oxepines 4.3 and 4.4 from 4.5. 
The ring expansion occurs as seen in chapter three, generating the oxepine 4.5. The acetate at C-5 is activated by the Lewis acid and departs generating a cation on the oxepane ring. This can then further react in two different ways. In pathway A, a deprotonation occurs at C-6 leading to the formation of a diene. This alkene is more reactive as it is also an enol ether. The enol ether is then protonated at C-5, forming an oxonium ion in the process. A second TMSallyl then attacks at C-6 forming the diallyl oxepine 4.3. In pathway $\mathrm{B}$ deprotonation at $\mathrm{C}-2$, which is adjacent to the allyl cation, generates the observed diene $\mathbf{4 . 4}$.

The distribution of the products from the two reactions performed gives some idea as to the kinetics of the reactions. Where there is an excess of nucleophile, the diallyl compound 4.3 is formed exclusively and pathway A is dominant. However when there is a limited amount of nucleophile present only compounds $\mathbf{4 . 4}$ and $\mathbf{4 . 5}$ are isolated and so pathway $\mathrm{B}$ is dominant.

The mechanism illustrated in Scheme 4.3 shows some similarities to that known to occur in the degradation of some naturally occurring linear polysaccharides such as pectin. ${ }^{95,96}$ Pectin is a polysaccharide that gives shape to plant cells and regulates water in the plant. It consists mostly of galactouronic acids with a mixture of other sugar moieties present as well. It is added extensively as a gelling or thickening agent to foodstuffs (mostly fruit jams and jellies). This polysaccharide can be degraded under both acidic and basic conditions to shorter oligosaccharides through the breaking of the glycosidic linkages. This proceeds through a $\beta$-elimination mechanism of which the basic version is shown in Scheme 4.4. 


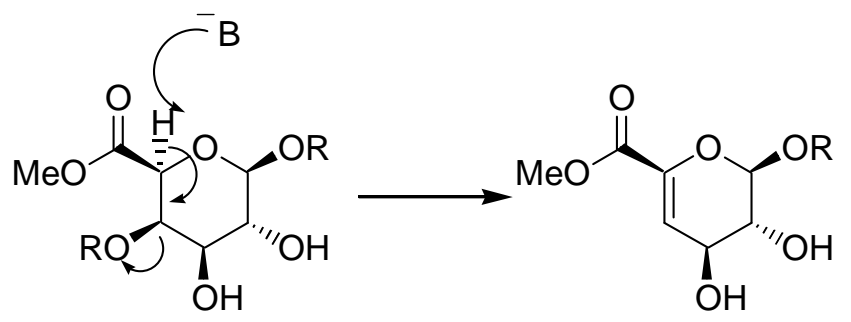

Scheme 4.4. Degradation of pectin to galacturonic acid. $\mathrm{R}$ is an oligosaccharide.

\subsection{Unexpected reaction products from ring expansions of cyclopropanated galactal 3.4}

\subsubsection{Reaction of TESH with cyclopropane 3.4}

Hoberg had shown that treatment of cyclopropane 1.61 with TESH led to the expected hydride substituted oxepine as shown in Scheme $4.5 .^{70}$

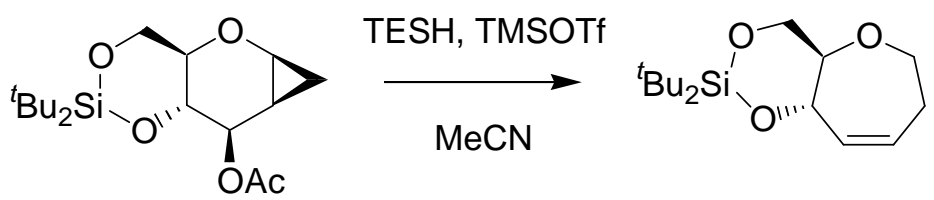

1.61

Scheme 4.5. Ring expansion of 1.61 with TESH.

However the reaction between cyclopropane 3.4 and TESH under the same reaction conditions did not give the expected oxepine. The product had an unusually high $\mathrm{R}_{\mathrm{f}}$ on TLC compared to the other oxepines synthesised. Furthermore, analysis of the ${ }^{1} \mathrm{H}$ NMR integration ratios suggested that there was a TES group present on the molecule. Also present were two broad multiplets both integrating for two protons at $\delta_{\mathrm{H}} 2.2$ and 2.5. The alkene protons were overlapping at $\delta_{\mathrm{H}} 5.85$ suggesting that both protons were in a similar chemical environment. HSQC spectral data revealed the presence of four methylene groups and only one oxymethine. This data suggested that there was an 
apparent deoxygenation, with the most likely location being at C-5 on the ring. The structure was therefore assigned as 4.7 (Scheme 4.6). High-resolution mass spectrometry confirmed the product had a mass of 401.29 Da consistent with this theory.

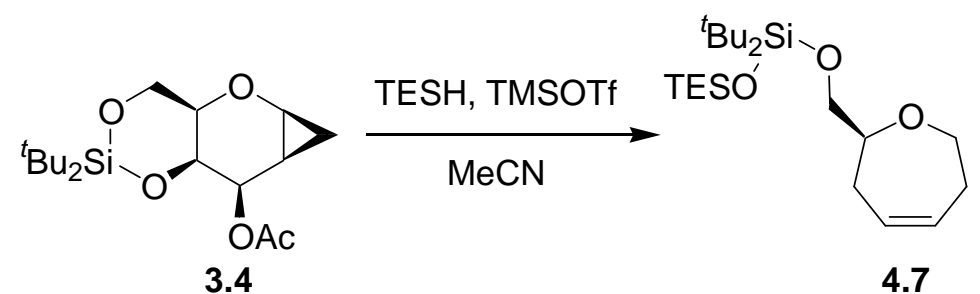

Scheme 4.6. Formation of oxepine 4.7 from cyclopropane 3.4.

It was postulated that the deoxygenation occured after the initial ring expansion where a TES cation co-ordinates to the axial oxygen at the $\mathrm{C}-5$ position. The actvated $\mathrm{C}-\mathrm{O}$ bond is then displaced by a hydride from the $\beta$ face forming the observed product (Scheme 4.7).

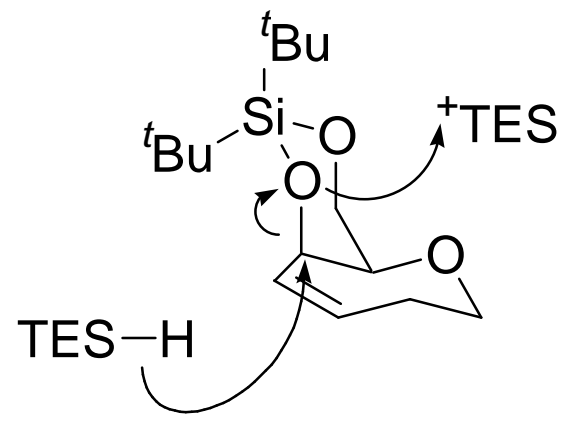

Scheme 4.7. Possible deoxygenation mechanism leading to oxepine 4.7.

\subsubsection{Investigation of the mechanism for the formation of oxepine 4.7}

To investigate further the mechanism for this rearrangement a deuterium labelling study was performed substituting TESD for TESH to provide deuteride as the nucleophile. Deuteride was used to obtain two pieces of information. The first was the stereoselectivity of the reaction as deuterium is expected to react in the same fashion as 
a hydride. The second is the regioselectivity of the reaction, which would confirm whether the mechanism postulated was correct. Deuterium is not decoupled in the standard ${ }^{13} \mathrm{C}$ NMR experiment; a carbon with a deuterium attached appears as a triplet with a coupling constant of $\sim 20 \mathrm{~Hz}$. TESD was prepared according to the method of Doyle and coworkers. ${ }^{97}$ The reaction with cyclopropane $\mathbf{3 . 4}$ generated a mixture of compounds that made initial interpretation of the reaction results difficult. Unfortunately, the mixture was impossible to separate into single pure components. Nonetheless, examination of the NMR data of the semi-purified products established that there were two different compounds present. Assignment of the major component of the mixture was undertaken first. The presence of both the TES and di-tert-butylsilyl groups was confirmed by ${ }^{29} \mathrm{Si}$ NMR $\left(\delta_{\mathrm{Si}} 9.2\right.$ and -21.0$)$. The ${ }^{13} \mathrm{C}$ NMR data revealed that the deuterium had initially intercepted the oxonium intermediate forming the ring expanded product. This was established by the observation of one-bond C-D coupling of the carbons at $\delta_{\mathrm{C}} \sim 70 \mathrm{ppm}$. Two such signals were identified indicating that both epimers were present. The integration of the proton signals associated with this carbon revealed the presence of deuterium and an $\alpha: \beta$ epimeric ratio of 1:1. Analysis of the HSQC-DEPT spectrum proved the presence of methylene groups on either side of the double bond with no evidence of C-D coupling in the ${ }^{13} \mathrm{C}$ spectrum. There was also a disconnection observed in both COSY and TOCSY 2D NMR experiments between the methylene at C-5 and the oxymethylene at C-7. Closer examination of the ${ }^{13} \mathrm{C}$ spectrum revealed the presence of a second deuterated carbon at $\delta_{\mathrm{C}} 80.2$ superimposed with a peak from the other compound in the sample. This corresponds to the C-6 position on the non-deuterated oxepine 4.7. Deuterium in this position instead of a proton would explain the lack of connectivity observed in the COSY and TOCSY data. The connectivity of the ${ }^{1} \mathrm{H}$ spin system was then constructed from a series of COSY correlations from the $\mathrm{C}-2$ methylene $\left(\delta_{\mathrm{H}} 2.49\right.$ and $\left.2.18, \delta_{\mathrm{C}} 35.6\right)$ to an oxymethine. This 
couples to two different proton resonances: an oxymethine $\left(\mathrm{C}-1: \delta_{\mathrm{H}} 4.02\right.$ and $3.41, \delta_{\mathrm{C}}$ 69.2) and to an alkenic methine (C-3: $\left.\delta_{\mathrm{H}} 5.81, \delta_{\mathrm{C}} 130.7\right)$. C-3 then couples to a second alkenic methine $\left(\mathrm{C}-4: \delta_{\mathrm{H}} 5.81, \delta_{\mathrm{C}} 129.4\right)$ to a methylene $\left(\mathrm{C}-5: \delta_{\mathrm{H}} 2.49\right.$ and $2.16, \delta_{\mathrm{C}}$ 35.6). There is also an oxymethylene $\left(\delta_{\mathrm{H}} 3.83\right.$ and $\left.3.54, \delta_{\mathrm{C}} 66.3\right)$ present that is assumed to be at C-7. Examination of the ${ }^{13} \mathrm{C}$ spectrum reveals a deuterated carbon $\left(\delta_{\mathrm{C}}\right.$ 80.2, t, $J=20.5 \mathrm{~Hz}$ ), at an appropriate chemical shift for C-6. The HMBC spectrum shows correlations from C-5 and C-7 into this centre which confirmed this assignment. From this information we deduced that the major compound $\mathbf{4 . 8}$ present was as depicted in Figure 4.6.

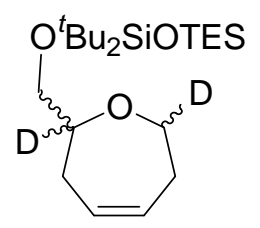

Figure 4.6. Deuterated compound oxepine 4.8 from TESD reaction.

This suggests that the mechanism that was proposed in Scheme 4.7 is incorrect, as it predicted the presence of the second deuterium would be at C-5 rather than the observed C-6.

The minor component in the mixture was then assigned to gain more information about a possible reaction mechanism. In the ${ }^{1} \mathrm{H}$ NMR of the mixture there was a single proton multiplet at $\delta_{\mathrm{H}} 6.0$ that showed no COSY correlations to any methylene signals in the ${ }^{1} \mathrm{H}$ spectra. However there were correlations from this proton into an $\mathrm{ABX}$ system at $\delta_{\mathrm{H}}$ 4.25, 3.95 and 3.72. Another correlation from a proton at $\delta_{\mathrm{H}} \sim 6 \mathrm{ppm}$ could be observed to a proton at $\delta_{\mathrm{H}} 4.42$ that showed no corresponding ${ }^{13} \mathrm{C}$ correlation on the HSQC. There was a deuterium-coupled carbon present in the ${ }^{13} \mathrm{C}$ spectrum in the correct region for a carbon attached to an ether oxygen. Analysis of the integrations of the ${ }^{1} \mathrm{H}$ signals 
suggested that there were four alkenic methines, two oxymethines and an oxymethylene, one of the oxymethylenes had a deuterium counected to it. The connectivity of the ${ }^{1} \mathrm{H}$ spin system was then constructed from a series of COSY correlations from the $\mathrm{C}-7\left(\delta_{\mathrm{H}} 3.97\right.$ and $\left.3.77, \delta_{\mathrm{C}} 65.1\right)$ to an oxymethine $\left(\mathrm{C}-6: \delta_{\mathrm{H}} 4.25, \delta_{\mathrm{C}}\right.$ 80.4) to an alkenic methine $\left(\mathrm{C}-5: \delta_{\mathrm{H}} 6.01, \delta_{\mathrm{C}} 125.6\right)$ to a second alkenic methine $(\mathrm{C}-4$ : $\left.\delta_{\mathrm{H}} 5.94, \delta_{\mathrm{C}} 126.2\right)$ to a third alkenic methine $\left(\mathrm{C}-3: \delta_{\mathrm{H}} 5.98, \delta_{\mathrm{C}} 135.4\right)$ to a forth alkenic methine $\left(\mathrm{C}-2: \delta_{\mathrm{H}} 5.93, \delta_{\mathrm{C}} 135.3\right)$ to an oxymethine $\left(\mathrm{C}-1: \delta_{\mathrm{H}} 4.43\right)$. This had no associated signal in the HSQC spectra. However in the ${ }^{13} \mathrm{C}$ NMR there was a deuterated carbon at $\delta_{\mathrm{C}} 70.2$ present as a triplet with a coupling constant of $21.1 \mathrm{~Hz}$ ). This assignment indicated the structure of this compound was as shown in Figure 4.7.

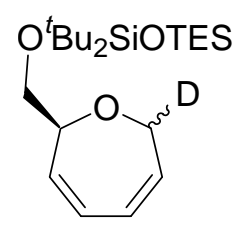

Figure 4.7. Minor product 4.9 from ring expansion with TESD.

Retrospectively, a minor product of the ring expansion of cyclopropane $\mathbf{3 . 4}$ with TESH (Section 4.2.1) was identified as the analogous deuterium free product using ${ }^{1} \mathrm{H}$ NMR of the crude reaction material. Its production in a far smaller ratio (approximately 6:1 vs the 2:1 observed here) had previously provided too little material for successful identification.

This information suggested that the mechanism was almost identical to that observed in Scheme 4.3. The expected oxepine 4.10 is formed in the initial steps (Scheme 4.8). 


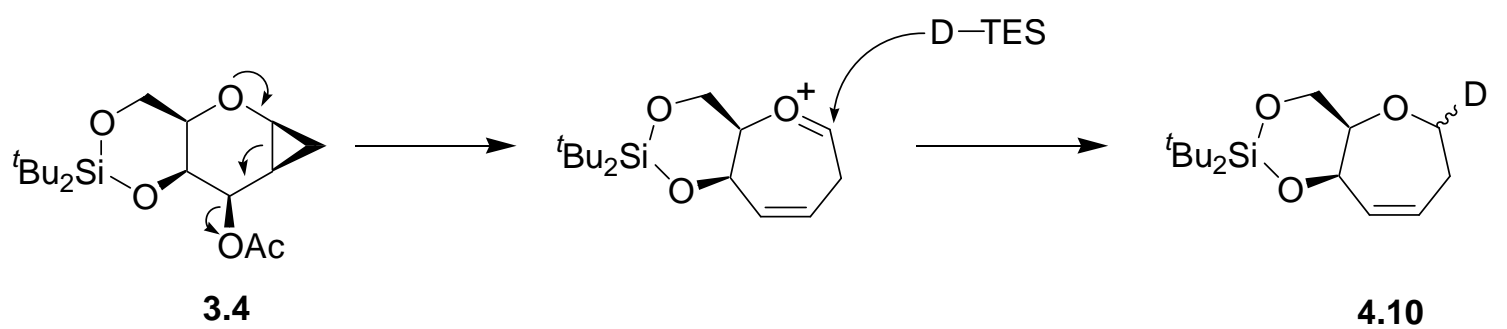

Scheme 4.8. Formation of deuterated oxepine 4.10.

The mechanism then proceeds as illustrated in Scheme 4.9. A TES cation coordinates to the C-5 oxygen. Thus activated it can then act as a leaving group. This again generates a cation at C-5. As seen before this can then proceed down one of two pathways. In Pathway A deprotonation at C-6 leads to the formation of an enol ether, that can then protonate at C-5, and form an oxonium ion, which is then intercepted by a second deuteride to form the major product oxepine 4.8. In pathway $\mathrm{B}$, deprotonation at the $\mathrm{C}-2$ adjacent to the resonance form of the cation at C-3 forms the diene 4.9. The intermediate oxepine $\mathbf{4 . 1 0}$ was not observed in the reaction products.

This mechanism is analogous to that proposed in Scheme 4.3. We can then propose that there is a unified mechanism for the reaction of this type of cyclopropanated glycal with an excess of the TMS nucleophile which then leads to the observed results. 


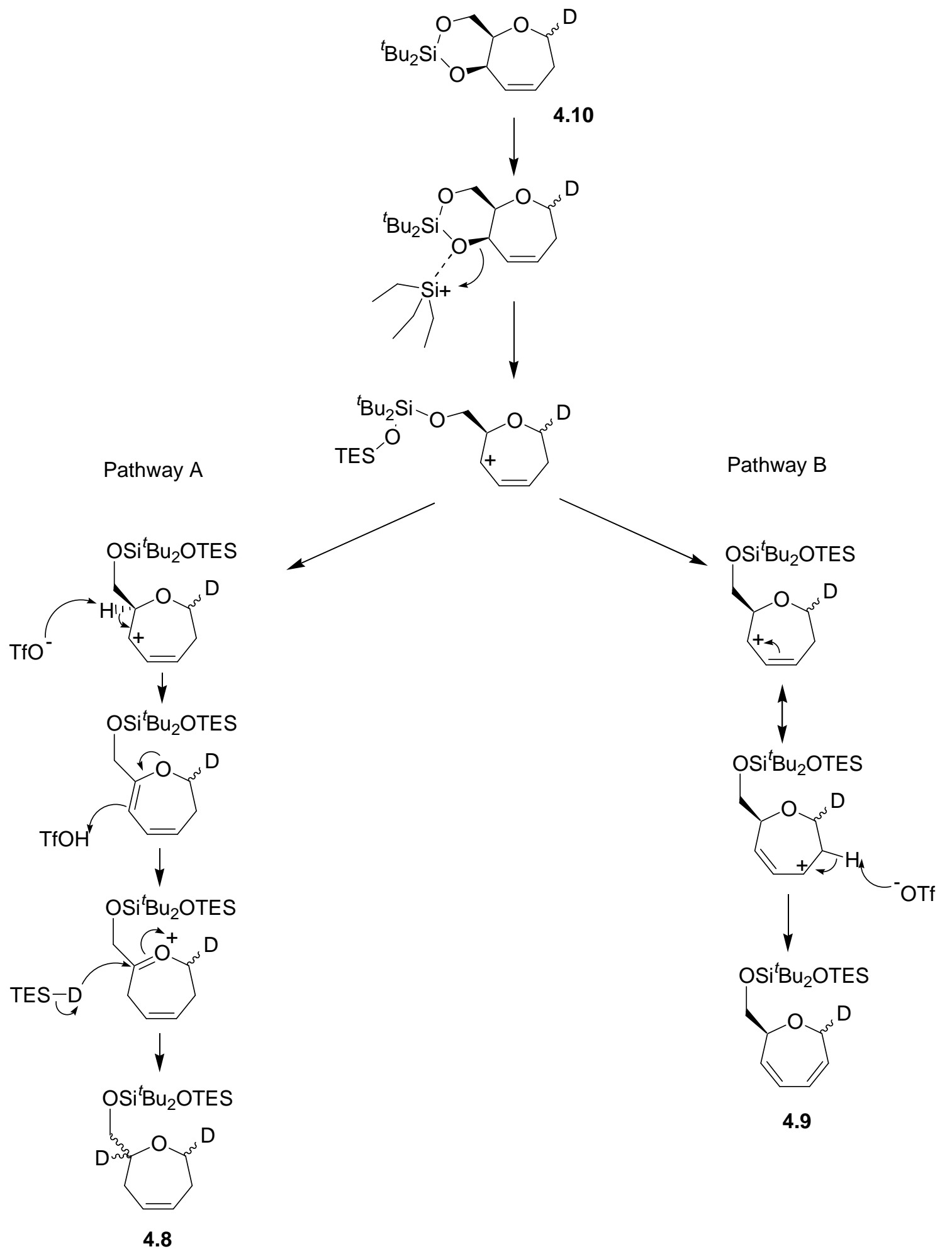

Scheme 4.9. Possible reaction mechanism to account for the formation of oxepines 4.8 and 4.9. 


\subsection{Summary}

In the course of the ring expansions of cyclopropanes 3.4 and 3.16 several unexpected reaction products were isolated. These were successfully characterized and mechanisms for their formation were postulated. The mechanisms were then tested by conducting trial reactions, and the results used to either confirm or modify the proposed mechanisms.

Ring expansion of cyclopropane 3.16 with nucleophiles 3.9 and 3.10 led to the generation of a diene species through a ring rearrangement initiated by the departure of the acetate group at C-5.

It was proposed that the products of the reaction between 3.16 and TMSallyl were formed through one of two competing mechanistic pathways. A further reaction to trap out possible reaction products indicated that the postulated mechanism was the correct one as all the reaction products could be accounted for. The expansion of cyclopropane 3.4 with either hydride or deuteride also appears to proceed through an analogous mechanism. 


\section{Chapter 5: Elaborations of oxepine 3.12}

Having discovered a high-yielding series of reactions that generate oxepines with high selectivity (Chapter 3), interest turned towards examining their utility as synthons. A series of simple transformations were initiated and the stereochemical outcomes and conformations of the products were investigated. Peczuh and co-workers have found from modelling studies and experimental observations that the underlying forces determining the preferred conformations of furanose and pyranose rings can generally be extended to the septanoses. ${ }^{98}$ The compounds synthesised in this chapter, while more correctly oxepanes, are in effect septanose analogues of carbohydrate derivatives. The conformational analysis of these compounds was intended to discover if Peczuh's extension was warranted to the oxepanes synthesised in this chapter.

NOE enhancements and vicinal ${ }^{1} \mathrm{H}^{1}{ }^{1} \mathrm{H}$ coupling constants were used to determine relative stereochemistry and conformation on the reaction products obtained. Based on the Karplus relationship and typical values observed in hexoses, large couplings (8-14 $\mathrm{Hz}$ ) were assumed to be due to 1,2-diaxial arrangements while axial-equatorial and equatorial-equatorial arrangements give rise to smaller couplings $(1-7 \mathrm{~Hz}$, with $2-3 \mathrm{~Hz}$ being more typical). ${ }^{84}$

Seven membered rings are known to exist in four principal conformers ${ }^{99}$ as shown in Figure 5.1.

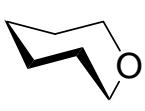

Chair

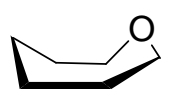

Boat

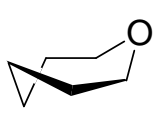

Twist-Chair

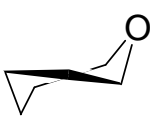

Skew

Figure 5.1. Known conformations of septanose rings. 
The predominant form in solution is the twist-chair, which accounts for $\sim 80 \%$ of the conformations of compounds with this type of structure. ${ }^{98}$

Oxepine 3.12 (Figure 5.2) was chosen as the substrate for the transformations. This compound was formed in the previous chapter's work and was chosen as the starting material for the following reasons: it was easily synthesised in high yield from the starting galactal 3.4, was stereochemically pure, and had functional groups on the extended side-chains that would be unreactive during reactions of the alkene but would provide handles for further manipulation.

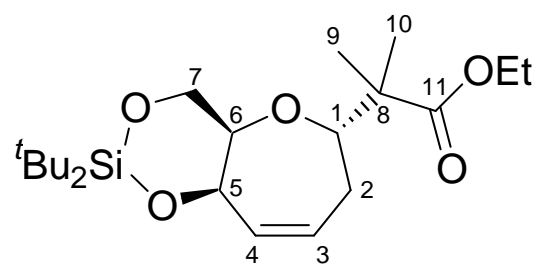

Figure 5.2. Oxepine 3.12 for elaboration showing ring-numbering system used for NMR analysis

One potential use of this oxepine synthon is in the synthesis of peloruside A analogues in a project to investigate structure-activity relationships. Peloruside A (Figure 5.3) is a novel secondary metabolite isolated from the New Zealand marine sponge Mycale hentscheli. $^{100}$ It shows potent microtubule stabilisation activity arresting cells in the G2-M phase of the cell cycle and inducing apoptosis. ${ }^{101}$

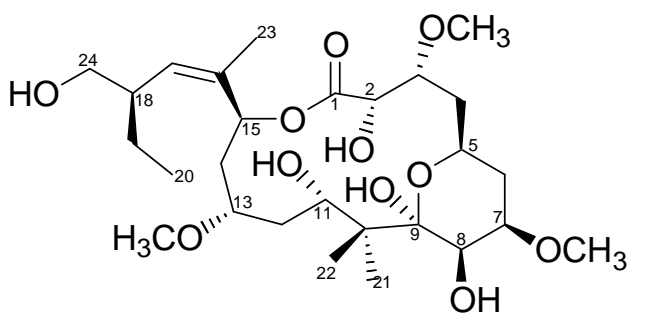

Figure 5.3. Structure of peloruside A. 
Its stereochemically challenging structure has been the subject of several total synthesis efforts. ${ }^{102,103}$ It is also an ideal candidate for the synthesis of analogue compounds to improve its efficiency as an anticancer compound. Overlaying the structure of a potential oxepine target on the pyranose substructure of peloruside A (Figure 5.4), it is apparent that there are many structural similarities which warrant the inclusion of an oxepane as a ring expanded analogue of the pyran ring in a synthesis program.

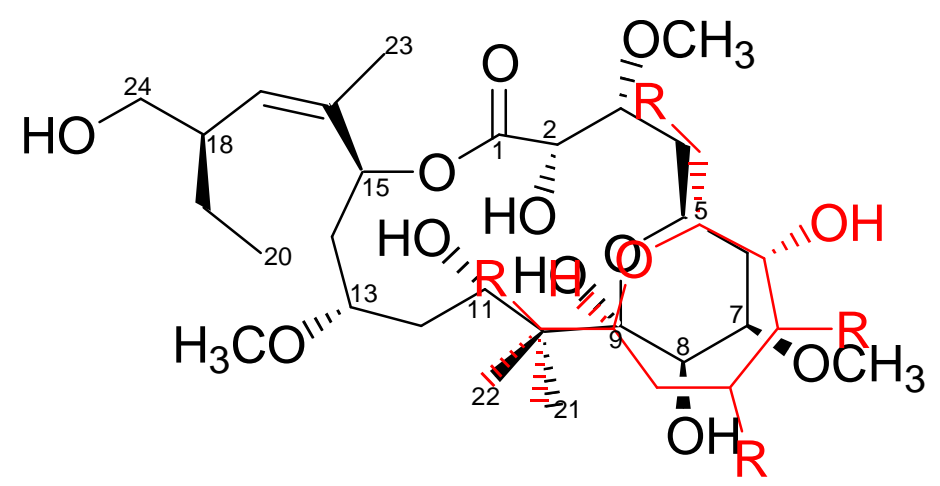

Figure 5.4. Oxepane derived from 3.12 overlayed on peloruside A.

The reactions investigated in the following sections were bromination, bromohydrin formation, epoxidation, dihydroxylation, hydroboration and reduction of the side chain ester group. These reactions were choosen as they provided a simple established set of transformations to probe the reactivity and elaboration potential of the oxepine ring.

\subsection{Halogen addition to oxepine 3.12}

\subsubsection{Bromination}

This simple reaction (Scheme 5.1) was performed first to check the reactivity of the oxepine and also to gain some initial information about the conformation of the resulting oxepane ring in solution. 


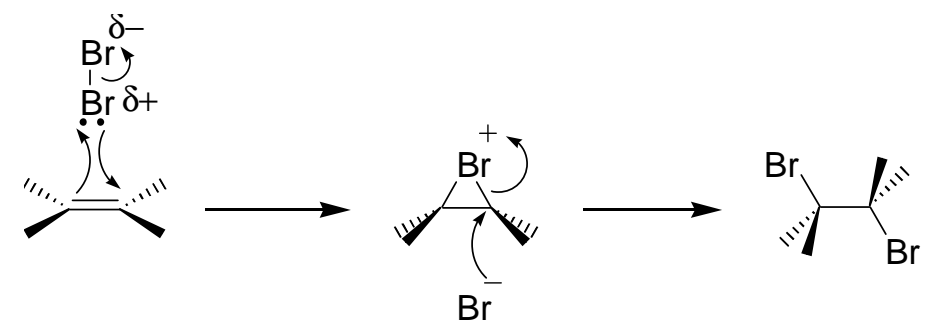

Scheme 5.1. Bromine addition to an alkene.

Addition of bromine to a solution of oxepine 3.12 in $\mathrm{CHCl}_{3}$ at ambient temperature for 30 minutes gave two products in $90 \%$ overall yield in a 2:1 ratio (entry one, table 5.1). Both isomers could be separated and purified by chromatography. Mass spectrometry of both revealed the presence of two bromine atoms, with ${ }^{1} \mathrm{H}$ and ${ }^{13} \mathrm{C}$ NMR indicating that the alkene was no longer present. However, the ${ }^{1} \mathrm{H}$ NMR of both compounds was congested between $\delta_{\mathrm{H}} 4$ and 5 and it was hard to distinguish individual signals. The spectral data set of the minor isomer showed better resolution in the ${ }^{1} \mathrm{H}$ NMR, enabling its assignment.

The structure of the minor isomer, oxepane 5.2, was assigned by ${ }^{1} \mathrm{H},{ }^{13} \mathrm{C}$ COSY, HSQCDEPT, HMBC, 1D TOCSY and NOE experiments. The presence of the gem-dimethyl ester group was confirmed by ${ }^{1} \mathrm{H}$ resonances consistent with two methyls $\left(\delta_{\mathrm{H}} 1.19\right.$ and $1.15)$ and an ethyl group $\left(\delta_{\mathrm{H}} 4.60\right.$ and $\left.\delta_{\mathrm{H}} 1.30\right)$. Similarly observed were ${ }^{13} \mathrm{C}$ resonances consistant with a carbonyl functionality $\left(\delta_{\mathrm{C}} 176.0\right)$ and a quaternary carbon center $\left(\delta_{\mathrm{C}}\right.$ 47.5). ${ }^{1} \mathrm{H}$ resonances also confirmed the presence of the di-tert-butyl silyl protecting group $\left(\delta_{\mathrm{H}} 1.18,18 \mathrm{H}\right)$. The HSQC-DEPT experiment, as well as establishing direct hydrogen-carbon connectivity, revealed the presence on the main ring of three oxymethines, two methines, an oxymethylene and a methylene which COSY correlations confirmed were all part of the same ${ }^{1} \mathrm{H}$ spin system. An HMBC correlation from the gem-dimethyl quaternary carbon was observed to the C-1 oxymethine $\left(\delta_{\mathrm{H}}\right.$ 4.41, $\delta_{\mathrm{C}}$ 78.8). The connectivity of the ${ }^{1} \mathrm{H}$ spin system was then constructed from a 
series of COSY and TOCSY correlations from the C-1 oxymethine proton to a methylene $\left(\mathrm{C}-2: \delta_{\mathrm{H}} 2.80\right.$ and $\left.1.97, \delta_{\mathrm{C}} 28.0\right)$ to a methine $\left(\mathrm{C}-3: \delta_{\mathrm{H}} 4.70, \delta_{\mathrm{C}} 48.5\right)$ to a second methine $\left(\mathrm{C}-4: \delta_{\mathrm{H}} 4.74, \delta_{\mathrm{C}} 57.6\right)$ to an oxymethine $\left(\mathrm{C}-5: \delta_{\mathrm{H}} 4.64, \delta_{\mathrm{C}} 79.8\right)$ to a second oxymethine $\left(\mathrm{C}-6: \delta_{\mathrm{H}} 4.07, \delta_{\mathrm{C}} 66.9\right)$ and finally to an oxymethylene $\left(\mathrm{C}-7: \delta_{\mathrm{H}}\right.$ 4.22 and $\left.4.12, \delta_{\mathrm{C}} 70.4\right)$. The conformation and relative stereochemistry around the oxepane ring were established from an analysis of NOE enhancements and ${ }^{1} \mathrm{H}-{ }^{1} \mathrm{H}$ coupling constants (Figure 5.5). The diagrams in this chapter illustrating the probable solution conformation are drawn with the ${ }^{1} \mathrm{H}$ axial-axial couplings in red, and the NOE enhancements in green.

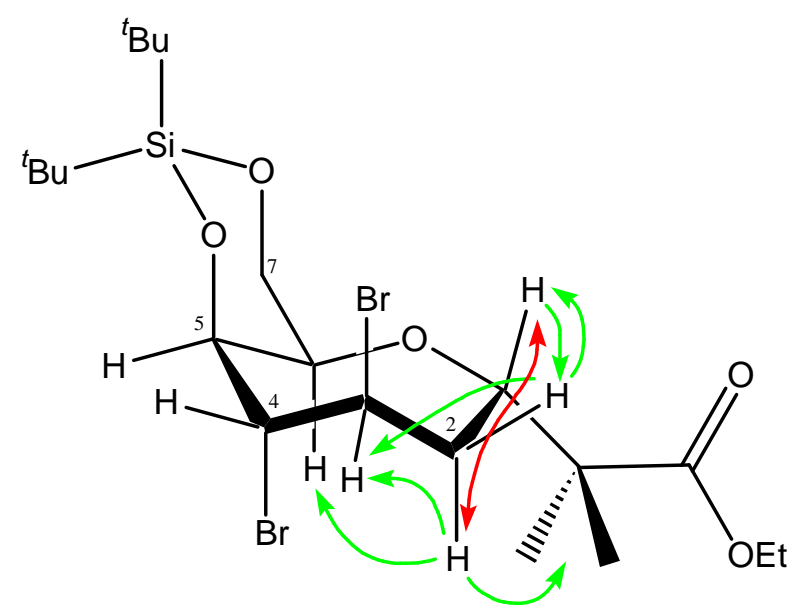

Figure 5.5. Minor isomer 5.2 from bromination showing NOE enhancements and ${ }^{1} \mathrm{H}$ axial-axial interactions.

$\mathrm{H}-1$ on the $\beta$ face of the molecule has a large coupling constant of $12.0 \mathrm{~Hz}$ shared with one of the protons at $\mathrm{H}-2\left(\delta_{\mathrm{H}} 2.80\right)$ indicating the anti-relationship of these two protons and establishes this methylene proton's position on the $\alpha$ face of the ring. Clear NOE enhancements were observed from $\mathrm{H}-2 \alpha$ to $\mathrm{H}-6$ and $\mathrm{H}-3$ which assigns these protons on the same side of the ring $(\alpha)$. These relationships establish the bromine at C-3 as being on the $\beta$ face. The second bromine at $\mathrm{C}-4$ is in an anti-relationship with its partner at $\mathrm{C}$ 3 and must therefore be on the $\alpha$ face. Coupling constants for H-3, H-4 and H-5 were 
all $<7 \mathrm{~Hz}$ assigning them in equatorial positions, consistent with axial bromines at C-3 and C-4. The perhaps somewhat surprising 1,2-axial positioning of the C-3 and C-4 bromines is consistent with a stable twist-chair conformation of the oxepane ring. This conformation is due to the presence of the large bulky $\alpha$ gem-dimethyl substituent at C-1 forcing the 2 bromines into axial orientations (Figure 5.5), and the structure stabilising effect of the di-tert-butylsilyl protecting group.

The major isomer from the reaction, oxepane 5.1, was similarly assigned by ${ }^{1} \mathrm{H},{ }^{13} \mathrm{C}$ COSY, HSQC-DEPT and HMBC. As was previously indicated, ${ }^{1} \mathrm{H}$ NMR spectra of this compound were particularly congested in the 4.2-4.0 ppm area (Figure 5.6 and 5.7).

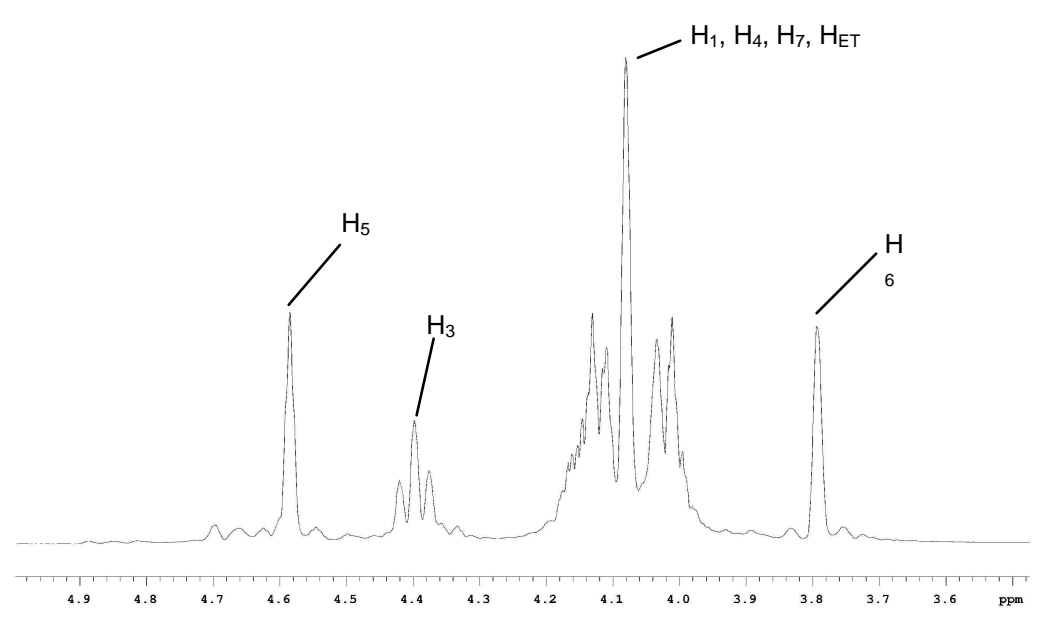

Figure 5.6. ${ }^{1} \mathrm{H}$ NMR spectrum of dibromide 5.1 in $\mathrm{CDCl}_{3}(500 \mathrm{MHz})$ showing the congested region between 4.2 and $4.0 \mathrm{ppm}$. 


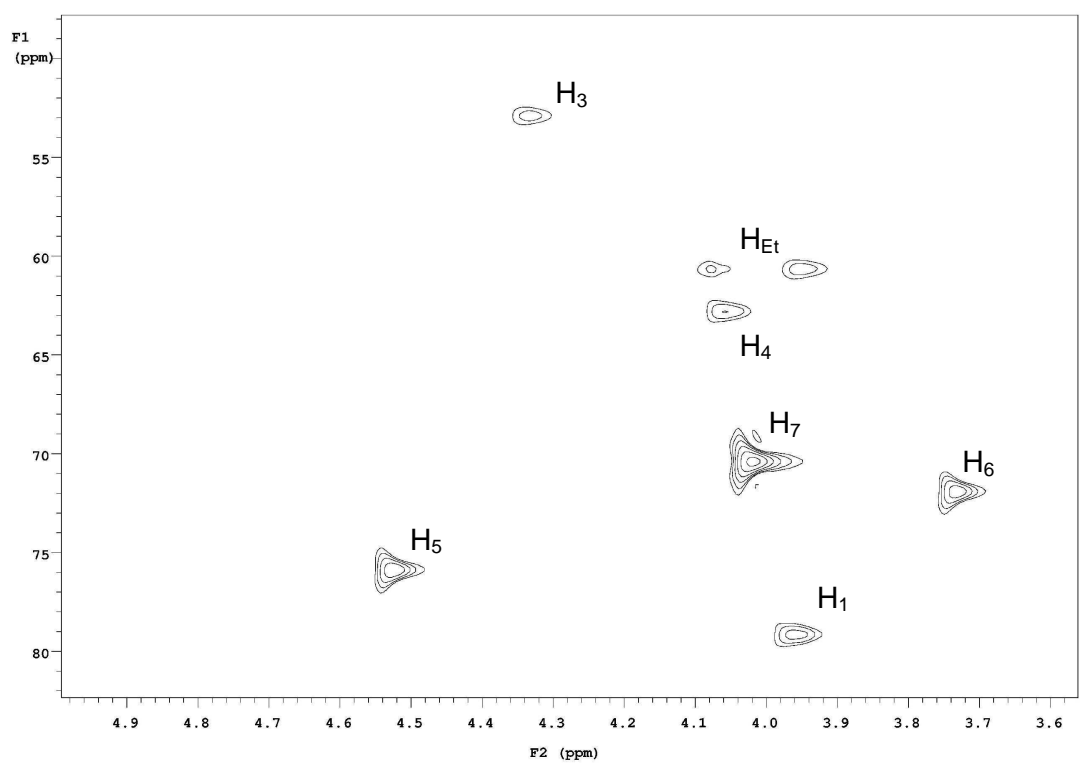

Figure 5.7. HSQC spectrum of dibromide 5.1 in $\mathrm{CDCl}_{3}$ showing overlapping proton signals between 4.2 and $4.0 \mathrm{ppm}$.

${ }^{1} \mathrm{H}$ and ${ }^{13} \mathrm{C}$ NMR again confirmed the presence of the silyl ether protecting group and also the gem-dimethyl-containing ester sidechain. As the C-6 and C-7 positions are not greatly effected by the substitution of the alkene, they were not included in the analysis of the NMR data of this compound. As the oxymethine at C-1 was not resolved, the ${ }^{1} \mathrm{H}$ spin system was constructed from COSY correlations starting with the C-2 methylene $\left(\delta_{\mathrm{H}} 2.57\right.$ and $\left.2.35, \delta_{\mathrm{C}} 38.6\right)$. These protons couple to an oxymethine $\left(\mathrm{C}-1: \delta_{\mathrm{H}} \sim 4.1, \delta_{\mathrm{C}}\right.$ 79.2) and a methine $\left(\mathrm{C}-3: \delta_{\mathrm{H}} 4.45, \delta_{\mathrm{C}} 52.9\right)$. The C-3 methine proton then couples to a second methine $\left(\mathrm{C}-4: \delta_{\mathrm{H}} \sim 4.2, \delta_{\mathrm{C}} 62.8\right)$ which correlates to an oxymethine $\left(\mathrm{C}-5: \delta_{\mathrm{H}}\right.$ $\left.4.64, \delta_{\mathrm{C}} 75.9\right)$ to a second oxymethine $\left(\mathrm{C}-6: \delta_{\mathrm{H}} 3.85, \delta_{\mathrm{C}} 71.9\right)$ and finally to an oxymethylene (C-7: $\left.\delta_{\mathrm{H}} \sim 4.15, \delta_{\mathrm{C}} 70.4\right)$. The conformation and relative stereochemistry around the oxepane ring were established from an analysis of NOE enhancements and ${ }^{1} \mathrm{H}-{ }^{1} \mathrm{H}$ coupling constants (Figure 5.8). The H-2 $\alpha$ proton $\left(\delta_{\mathrm{H}} 2.35\right)$ could be assigned from NOE enhancements to H-6 and the gem-dimethyl protons. The H-2 $\alpha$ proton 
appears as a doublet of triplets with coupling constants of 15.7 and $11.5 \mathrm{~Hz}$, indicating that it is in an anti relationship with both $\mathrm{H}-1$ and $\mathrm{H}-3$ which places the bromine at $\mathrm{C}-3$ in an equatorial orientation. H-3 also appears as a doublet of triplets with coupling constants of 11.0 and $1.7 \mathrm{~Hz}$ which place it in an anti relationship with $\mathrm{H}-2 \alpha$ and also H-4. H-4's axial position is confirmed by an NOE enhancement from $\mathrm{H}-2 \alpha$, placing the bromine at C-4 also in an equatorial orientation. Models suggested that the most probable conformation for this molecule was as a twist chair (Figure 5.8).

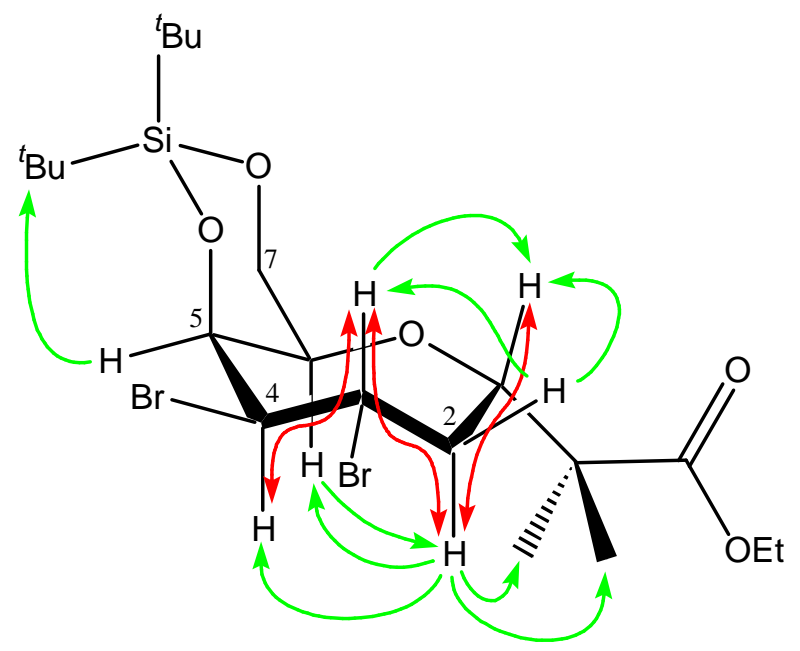

Figure 5.8. Major isomer 5.1 from bromination showing NOE enhancements and anti ${ }^{1} \mathrm{H}$ axial-axial couplings.

Dibromide 5.1 was subsequently deprotected with TBAF to give oxepane $\mathbf{5 . 3}$ in a 58\% yield (Figure 5.9). This reaction had a twofold purpose; i) to determine if the removal of the silyl group under normal conditions resulted in any unexpected rearrangements and ii) to see if any more information could be obtained about the conformation in solution as $\mathbf{5 . 1}$ and $\mathbf{5 . 2}$ had a substantial number of overlapping signals. 


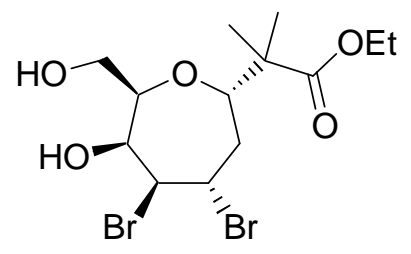

Figure 5.9. Diol 5.3.

The structure of oxepane $\mathbf{5 . 3}$ was assigned by ${ }^{1} \mathrm{H},{ }^{13} \mathrm{C}$ COSY, HSQC-DEPT and NOESY. Analysis of the ${ }^{1} \mathrm{H}$ NMR revealed that removal of the protecting group had resolved most of the protons on the oxepane ring (Figures 5.10 and 5.11). ${ }^{1} \mathrm{H}$ and ${ }^{13} \mathrm{C}$ NMR confirmed the presence of the gem-dimethyl-containing ester sidechain. The connectivity of the ${ }^{1} \mathrm{H}$ spin system was then constructed from a series of COSY correlations from the C-1 oxymethylene $\left(\delta_{\mathrm{H}} 4.34, \delta_{\mathrm{C}} 78.9\right)$ to a methylene $\left(\mathrm{C}-2: \delta_{\mathrm{H}} 2.50\right.$ and $\left.2.23, \delta_{\mathrm{C}} 39.7\right)$ to a methine $\left(\mathrm{C}-3: \delta_{\mathrm{H}} 4.51, \delta_{\mathrm{C}} 53.7\right)$ to a second methine $\left(\mathrm{C}-4: \delta_{\mathrm{H}}\right.$ $\left.4.25, \delta_{\mathrm{C}} 63.5\right)$ to an oxymethine $\left(\mathrm{C}-5 ; \delta_{\mathrm{H}} 4.43, \delta_{\mathrm{C}} 76.0\right)$ to a second oxymethine $\left(\mathrm{C}-6: \delta_{\mathrm{H}}\right.$ $\left.3.84, \delta_{\mathrm{C}} 74.9\right)$ and finally to an oxymethylene $\left(\mathrm{C}-7: \delta_{\mathrm{H}} 3.78\right.$ and $\left.3.71, \delta_{\mathrm{C}} 65.4\right)$. The conformation and relative stereochemistry around the oxepane ring were established from an analysis of NOE enhancements and ${ }^{1} \mathrm{H}-{ }^{1} \mathrm{H}$ coupling constants (figure 5.12). H1 has a large coupling constant of $10.5 \mathrm{~Hz}$ with $\mathrm{H}-2 \alpha\left(\delta_{\mathrm{H}} 2.23\right)$ placing them in an anti relationship.

H-2 $\alpha$ then shows NOE enhancements to H-4 and H-6. H-2 $\alpha$ in turn has a large coupling constant of $12.2 \mathrm{~Hz}$ with $\mathrm{H}-3$ placing these protons also in an anti relationship. Finally there is a large coupling constant of $10.7 \mathrm{~Hz}$ placing $\mathrm{H}-4$ in an axial orientation as well. This places both bromines in equatorial orientations, at C-3 $\alpha$ and at C-4 $\beta$, which confirms the structure of the parent dibromide 5.2. 


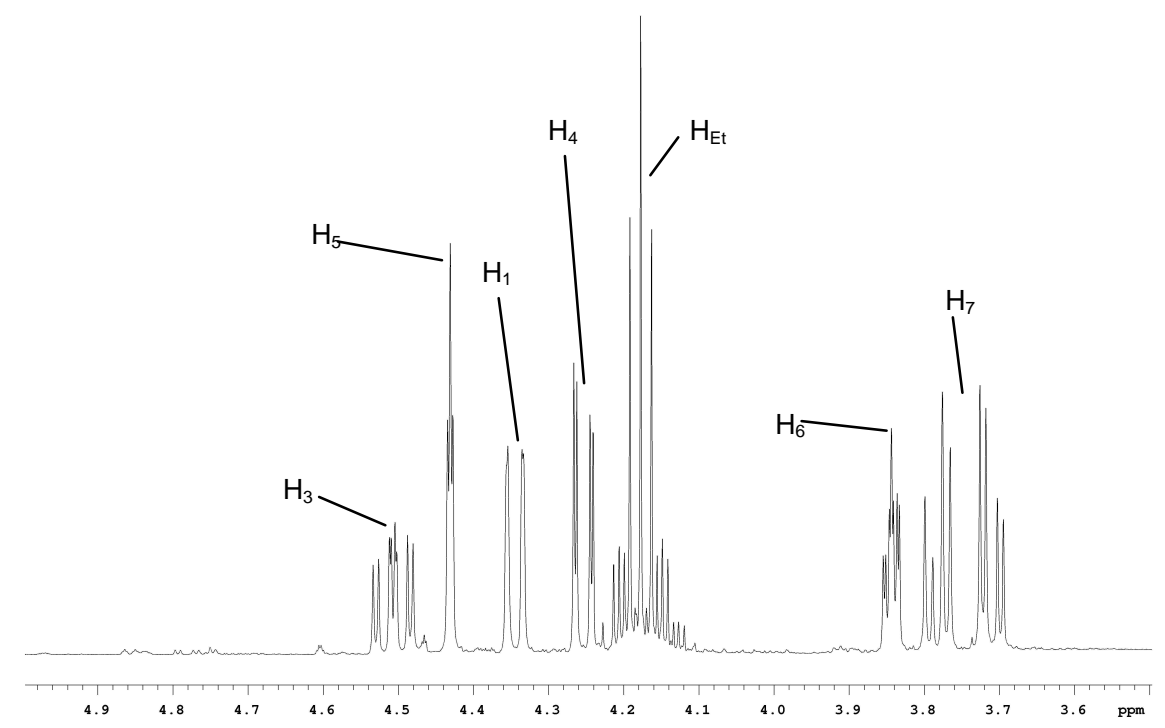

Figure 5.10. ${ }^{1} \mathrm{H}$ NMR spectrum of dibromide 5.3 in $\mathrm{CDCl}_{3}(500 \mathrm{MHz})$ demonstrating resolution of the previously congested area between $\delta_{\mathrm{H}} 4.2$ and 4.0.

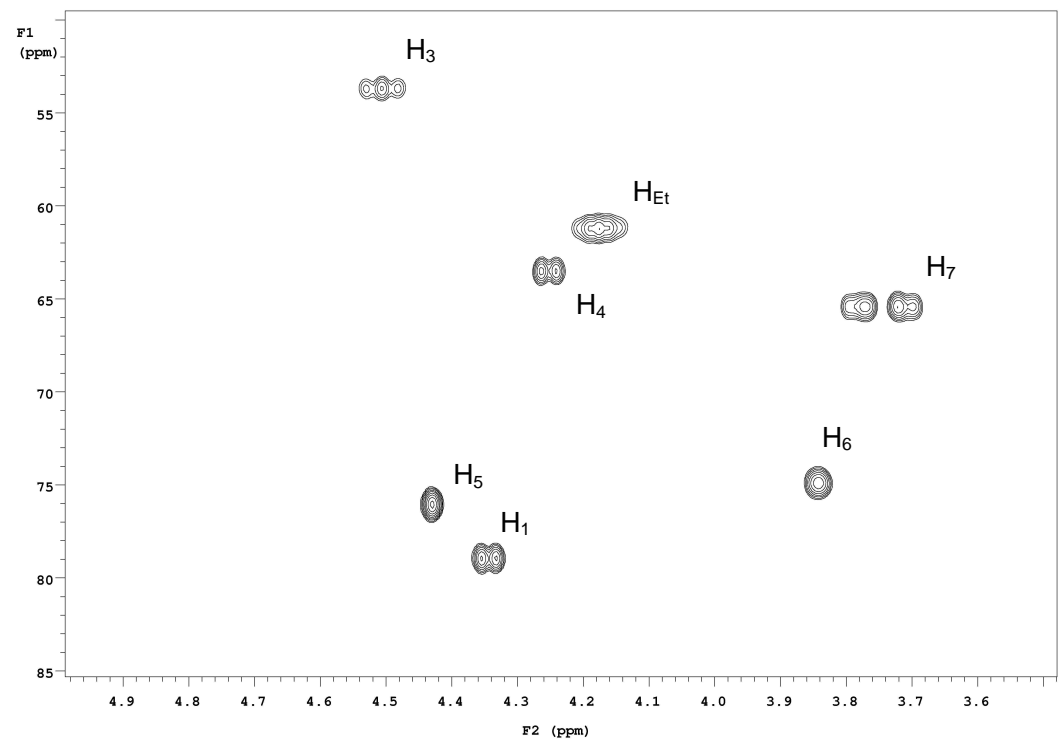

Figure 5.11. HSQC spectrum of dibromide 5.3 in $\mathrm{CDCl}_{3}$ showing resolution of overlapping proton signals. 
Examination of ball and stick models indicate that the solution conformation is the twist-chair form (Figure 5.12).

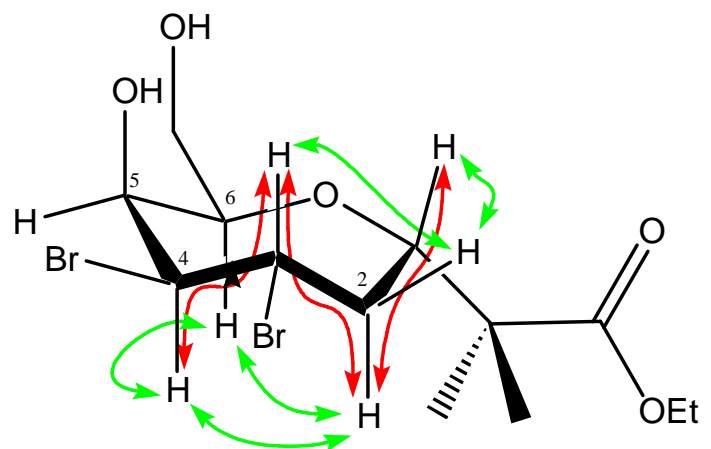

Figure 5.12. Dibromide 5.3 showing NOESY enhancements and ${ }^{1} \mathrm{H}$ axial-axial interactions.

\subsubsection{Bromohydrin formation}

Bromohydrins are an important precursor to several other functionalities, for instance epoxides. ${ }^{104}$ Reaction of oxepine $\mathbf{3 . 1 2}$ with NBS in water gave the bromohydrin $\mathbf{5 . 4}$ in a $77 \%$ yield as a 4:1 mixture with its minor isomer (entry two, table 5.1). The ${ }^{1} \mathrm{H}$ NMR of the major product was too congested to allow complete structural assignment, with the presence of three methines and two oxymethines between $\delta_{\mathrm{H}} 4.0$ and $4.2 \mathrm{ppm}$. To gain better ${ }^{1} \mathrm{H}$ NMR resolution bromohydrin $\mathbf{5 . 4}$ was acetylated to give oxepane 5.10. This derivatisation improved the resolution of the ${ }^{1} \mathrm{H}$ NMR and it was possible to assign the parent structure based on the 1D and 2D NMR data. Signals in the ${ }^{1} \mathrm{H}$ and ${ }^{13} \mathrm{C}$ NMR confirmed the presence of the gem-dimethyl containing ester group, and the di-tertbutyl silyl protecting group. As the oxymethine at C-1 was obscured in the ${ }^{1} \mathrm{H}$ spectrum, the ${ }^{1} \mathrm{H}$ spin system was constructed from COSY correlations starting with the C-2 methylene $\left(\delta_{\mathrm{H}} 1.96, \delta_{\mathrm{C}} 33.3\right)$. These protons couple to an oxymethine $\left(\mathrm{C} 1: \delta_{\mathrm{H}} \sim 4.2\right.$, $\left.\delta_{\mathrm{C}} 78.0\right)$ and to a second oxymethine $\left(\mathrm{C}-3: \delta_{\mathrm{H}} 5.14, \delta_{\mathrm{C}} 58.7\right)$. H-3 couples to a methine $\left(\mathrm{C}-4: \delta_{\mathrm{H}} \sim 4.1, \delta_{\mathrm{C}} 58.7\right)$, which in turn couples to an oxymethine $\left(\mathrm{C}-5: \delta_{\mathrm{H}} 4.53, \delta_{\mathrm{C}} 74.3\right.$ ppm). These correlations confirm the substitution pattern as 3-hydroxy-4-bromo. As 
the C- 6 and C-7 positions are not effected by the substitution of the alkene they were not included in subsequent analysis of the NMR data for the rest of this chapter. The conformation and relative stereochemistry around the oxepane ring were established from an analysis of NOE enhancements and ${ }^{1} \mathrm{H}-{ }^{1} \mathrm{H}$ coupling constants (Figure 5.13). Only two of the protons could be clearly identified: H-3 and H-5. H-3 appears as a triplet of doublets with coupling constants of 10.4 and $1.6 \mathrm{~Hz}$. The two large couplings indicate $\mathrm{H}-3$ is in an anti relationship with both $\mathrm{H}-2 \alpha$ and $\mathrm{H}-4$, placing both the hydroxyl at C-3 and the bromine at C-4 in equatorial orrientations. An NOE enhancement was observed from H-3 to H-1 placing this proton on the $\beta$ face. These relationships establish the stereochemistry of the addition product as, with the 3hydroxy group on the $\alpha$ face and the 4-bromo on the $\beta$ face.

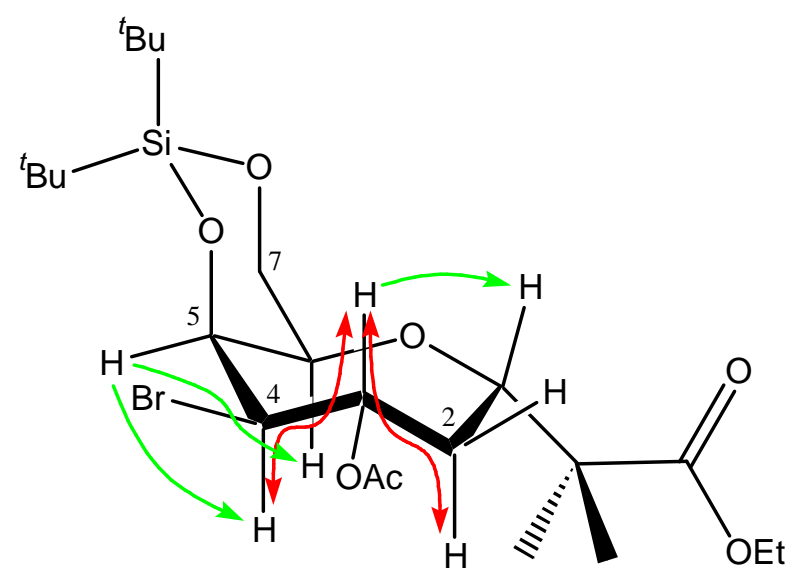

Figure 5.13. Major isomer 5.10 from acetylation of bromohydrin $\mathbf{5 . 4}$ showing NOE enhancements and ${ }^{1} \mathrm{H}$ axial-axial interactions.

Examination of models coupled with this data allowed assignment of the solution conformation as a twist-chair (Figure 5.13). 


\subsubsection{Mechanism of halogen addition to 3.12}

The results from the formation of 5.1, 5.2 and $\mathbf{5 . 4}$ were intuitively hard to explain. At first glance the major isomer would be expected to be that with the initial bromine attack from the $\alpha$ face due to steric constraints precluding attack from the $\beta$ face. However a closer investigation of the mechanism reveals that the initial bromine attack is electrophilic in nature. Thus the results can be rationalised if the initial bromine attack is directed by the lone electron pair on the C-5 oxygen leading to an intermediate state where the bromine is co-ordinated to the oxygen on the $\beta$ face as shown in Scheme 5.2. The nucleophile $\left(\mathrm{Br}^{-}\right.$or $\left.\mathrm{OH}^{-}\right)$is then directed to attack from the $\alpha$ face.

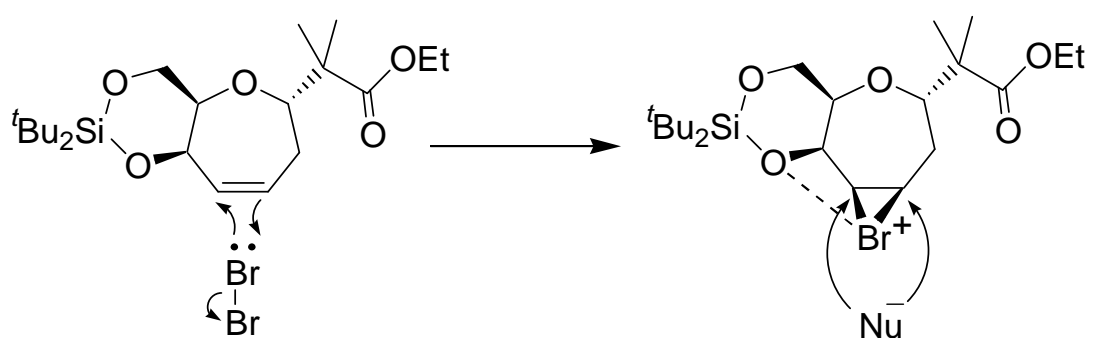

Scheme 5.2. Brominated intermediate co-ordinating to the C-5 oxygen followed by attack of nucleophile.

\subsection{Epoxidation of oxepine 3.12 and formation of derivatives}

\subsubsection{Epoxidation of oxepine 3.12}

One of the most useful reactions to increase the functionality of an alkene is epoxidation. Epoxides are important intermediates in many synthetic routes on the way to more complex molecules. While stereoselective methods of epoxidation are now commonplace, ${ }^{105}$ a simple expoxidation method was chosen to examine the reactivity of the oxepine and to determine the stereochemical outcome of the undirected reaction. 
Epoxidation of oxepine 3.12 with m-CPBA (the Prilezhaev reaction, ${ }^{106}$ Scheme 5.3) gave only one product, oxepine $\mathbf{5 . 5}$ in a $65 \%$ yield (entry three, table 5.1).

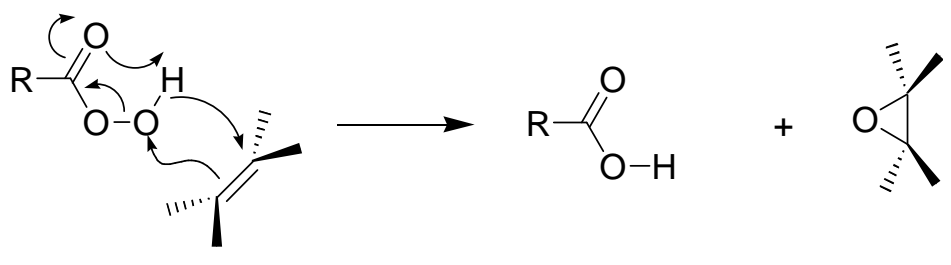

Scheme 5.3. Epoxidation mechanism with a peracid.

The structure was assigned from the 1D and 2D NMR data. Signals in the ${ }^{1} \mathrm{H}$ and ${ }^{13} \mathrm{C}$ NMR confirmed the presence of the gem-dimethyl-containing ester group, and the ditert-butylsilyl protecting group. The connectivity of the ${ }^{1} \mathrm{H}$ spin system was then constructed from a series of COSY correlations from the C-1 oxymethine $\left(\delta_{\mathrm{H}} 4.23, \delta_{\mathrm{C}}\right.$ 78.3) to a methylene $\left(\mathrm{C}-2: \delta_{\mathrm{H}} 2.13\right.$ and $\left.1.91, \delta_{\mathrm{C}} 27.1\right)$ to an oxymethine $\left(\mathrm{C}-3: \delta_{\mathrm{H}} 3.28\right.$, $\delta_{\mathrm{C}}$ 55.6) to a second oxymethine $\left(\mathrm{C}-4: \delta_{\mathrm{H}} 3.05, \delta_{\mathrm{C}} 56.6\right)$ and finally to a third oxymethine $\left(\mathrm{C}-5: \delta_{\mathrm{H}} 4.60, \delta_{\mathrm{C}} 74.1\right)$.

The conformation and relative stereochemistry around the oxepane ring were established from an analysis of NOESY enhancements and ${ }^{1} \mathrm{H}-{ }^{1} \mathrm{H}$ coupling constants. $\mathrm{H}-1$ has a large coupling constant of $12.5 \mathrm{~Hz}$ with $\mathrm{H}-2 \alpha\left(\delta_{\mathrm{H}} 1.91\right)$ confirming their anti relationship. H-2 $\alpha$ shows NOE enhancements to H-6. H-3 has coupling constants of 8.5 and $4.7 \mathrm{~Hz}$, which place it syn to $\mathrm{H}-4$ and in an eclipsed relationship with $\mathrm{H}-2 \beta\left(\delta_{\mathrm{H}}\right.$ 2.14). NOE enhancements are observed from $\mathrm{H}-2 \beta$ to $\mathrm{H}-3$ and $\mathrm{H}-1$ placing these three protons on the $\beta$ face. The position of $\mathrm{H}-3$ indicates that the epoxide is present on the $\alpha$ face and as the mechanism of epoxide addition exclusively gives a syn product, the epoxide is on the $\alpha$ face, consistent with the observed coupling patterns and NOE data. 


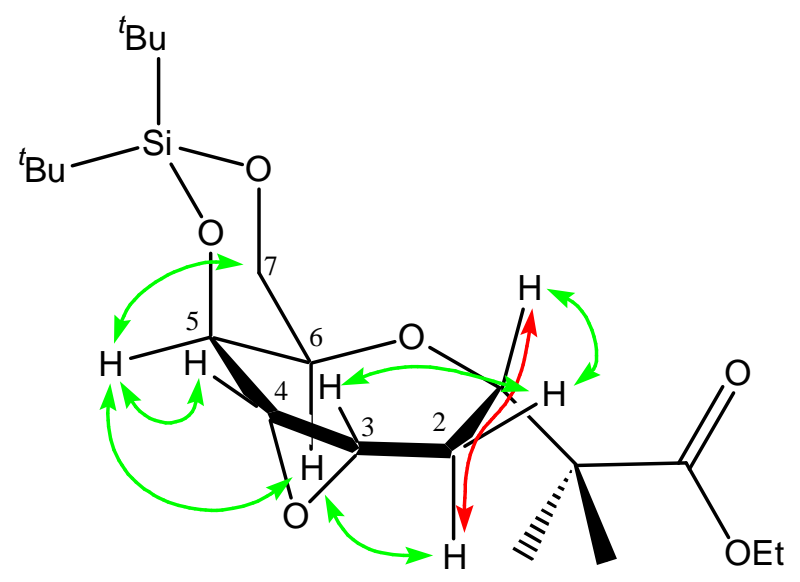

Figure 5.14. Structure of epoxide 5.5 showing NOESY enhancements and ${ }^{1} \mathrm{H}$ axial-axial interactions.

\subsubsection{Addition of sodium azide to epoxide 5.5}

As the epoxide was considered a stepping-stone on the path to more complex molecules, a further simple elaboration was performed. Treatment of epoxide $\mathbf{5 . 5}$ with sodium azide and ammonium chloride in a mixture of 8:1 MeOH: $\mathrm{H}_{2} \mathrm{O}$ heated at reflux overnight gave oxepine $\mathbf{5 . 6}$ as a single product in $63 \%$ yield (entry four, table 5.1 ). The ${ }^{1}$ H NMR spectra was very congested, with four methines and two oxymethylenes in the region between $\delta_{\mathrm{H}} 4.2$ and 4.0, which made it very difficult to assign the structure or the stereochemistry. Acetylation of oxepane 5.6 to compound 5.11 resolved some of the ring protons and made it possible to identify the structure and stereochemistry of the product. Again signals in the ${ }^{1} \mathrm{H}$ and ${ }^{13} \mathrm{C}$ NMR confirmed the presence of the gemdimethyl-containing ester group, and the di-tert-butylsilyl protecting group. As the oxymethine proton of $\mathrm{C}-1$ was obscured in the ${ }^{1} \mathrm{H}$ spectrum, connectivity of the ${ }^{1} \mathrm{H}$ spin system was then constructed from a series of COSY correlations from the C-2 methylene $\left(\delta_{\mathrm{H}} 2.17\right.$ and $\left.1.75, \delta_{\mathrm{C}} 26.7\right)$ to an oxymethine $\left(\mathrm{C}-1: \delta_{\mathrm{H}} \sim 4.15, \delta_{\mathrm{C}} 76.7\right)$ and to a methine $\left(\mathrm{C}-3: \delta_{\mathrm{H}} 3.95, \delta_{\mathrm{C}} 58.8\right)$. H-3 correlates to an oxymethine $\left(\mathrm{C}-4: \delta_{\mathrm{H}} 5.33, \delta_{\mathrm{C}}\right.$ 73.7) to a second oxymethine $\left(\mathrm{C}-5: \delta_{\mathrm{H}} 4.25, \delta_{\mathrm{C}} 75.9\right)$. This assigns the regioselectivity of the azide addition to $\mathrm{C}-3$. The conformation and relative stereochemistry around the 
oxepane ring were established from an analysis of NOE and NOESY enhancements and ${ }^{1} \mathrm{H}-{ }^{1} \mathrm{H}$ coupling constants. A clear NOE enhancement can be seen from $\mathrm{H}-6$ to $\mathrm{H}-2 \alpha$ $\left(\delta_{\mathrm{H}} 2.17\right)$ assigning this proton to the same face. $\mathrm{H}-2 \alpha$ has coupling constants of 12.8 and $3.4 \mathrm{~Hz}$ to $\mathrm{H}-1$ and $\mathrm{H}-3$ respectively. This assigns $\mathrm{H}-1$ as axial and $\mathrm{H}-3$ as equatorial. H-3 also has NOE enhancements to $H-2 \alpha, H-2 \beta$ and $H-4$ which positions this proton in a syn orientation vis these three protons. The coupling constants of $\mathrm{H}-4$ and $\mathrm{H} 5$ are $<6.5 \mathrm{~Hz}$ which places them in equatorial positions as well. Using this information, models indicate that the solution configuration is a twist-chair form (Figure $5.15)$

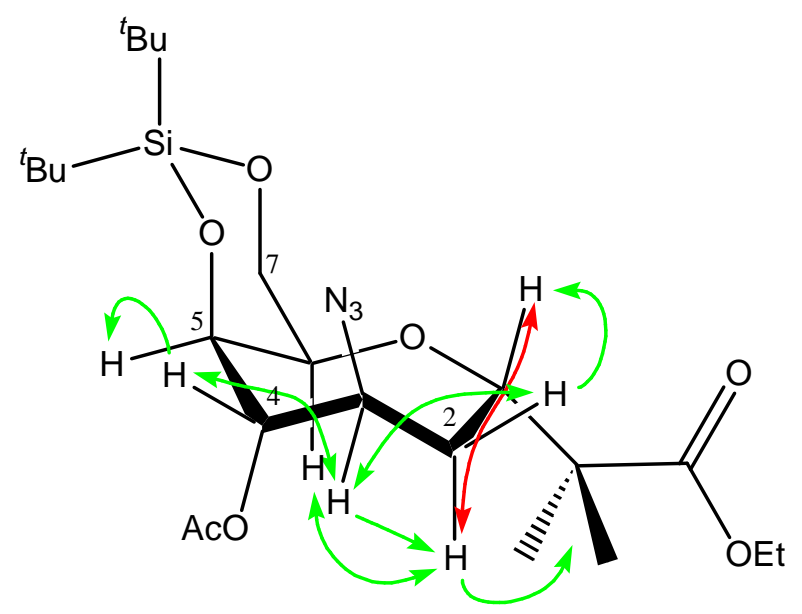

Figure 5.15. Structure of actylated azide 5.11 showing NOE and NOESY enhancements and ${ }^{1} \mathrm{H}$ axialaxial interactions.

As the hydroxyl group formed at C-4 remains $\alpha$, the azide adds in an anti fashion to give the C-3 $\beta$ substituent. The azide addition therefore occurs at the least sterically and electronically hindered carbon as shown in Figure 5.16.<smiles>CCOC(=O)C(C)(C)[C@@H]1CC(N)[C@H](O)[C@H]2O[SiH](C)CC[C@H]2O1</smiles>

Figure 5.16. Stereochemistry of the azide addition to epoxide $\mathbf{5 . 5}$. 


\subsection{Dihydroxylation of oxepine 3.12}

Osmium tetroxide catalyses the cis dihydroxylation of alkenes in the presence of water $^{107}$ (Scheme 5).

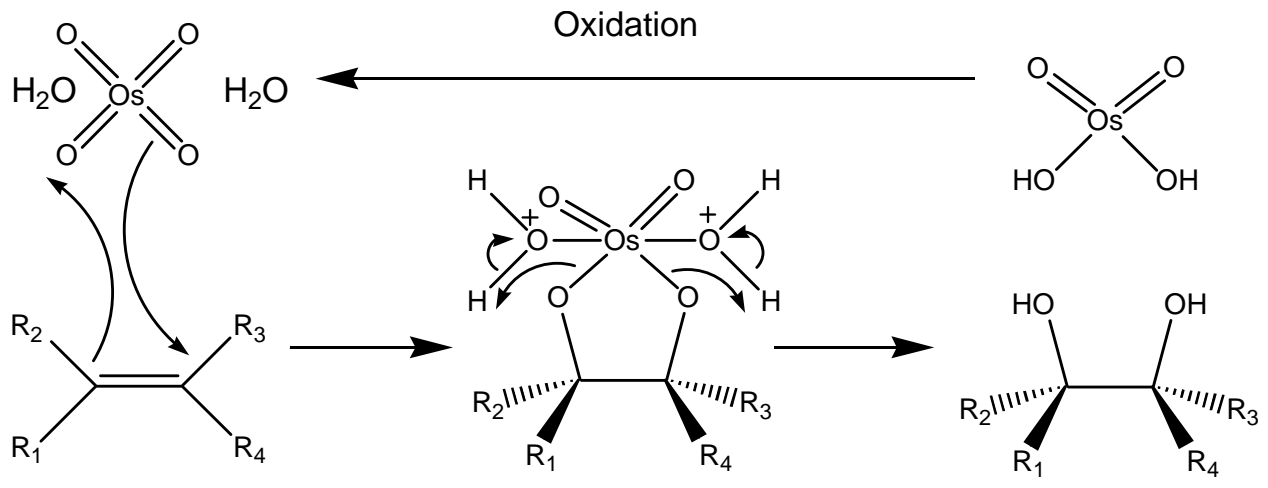

Scheme 5.4. Mechanism for osmium tetroxide dihydroxylation.

The reaction can be made stereoselective by the application of the Sharpless method. ${ }^{108}$ However, this modification was deemed unnecessary given the exploratory nature of the investigations. Reaction of oxepine $\mathbf{3 . 1 2}$ with osmium tetroxide in a 1:1 mix of diethyl ether and water at ambient temperature for one hour gave only one product, diol $\mathbf{5 . 7}$, in a $59 \%$ yield (entry five, table 5.1).

Again, the ${ }^{1} \mathrm{H}$ NMR was too congested to allow detailed assignment of any of the signals with four methines and two oxymethylenes in the region $\delta_{\mathrm{H}}$ 3.9-4.15. Acetylation of the diol $\mathbf{5 . 7}$ gave oxepane $\mathbf{5 . 1 2}$ in a 79\% yield. This derivatisation was successful in dispersing the oxepane ring peaks sufficiently for the stereochemistry to be determined. Connectivity of the ${ }^{1} \mathrm{H}$ spin system was then constructed from a series of COSY correlations from the C-2 methylene $\left(\delta_{\mathrm{H}} 2.49,1.67, \delta_{\mathrm{C}} 27.0\right)$. This couples to an oxymethine $\left(\mathrm{C}-1: \delta_{\mathrm{H}} \sim 4.15, \delta_{\mathrm{C}} 78.5\right)$ and a second oxymethine $\left(\mathrm{C}-3: \delta_{\mathrm{H}} 5.19\right.$, $\left.\delta_{\mathrm{C}} 70.6\right)$. C-3 then couples to an oxymethine $\left(\mathrm{C}-4: \delta_{\mathrm{H}} 5.24, \delta_{\mathrm{C}} 74.0\right)$ which couples to a 
second oxymethine $\left(\mathrm{C}-5: \quad \delta_{\mathrm{H}} 3.99, \quad \delta_{\mathrm{C}} 73.5\right)$. The conformation and relative stereochemistry around the oxepane ring were established from an analysis of NOE enhancements and ${ }^{1} \mathrm{H}-{ }^{1} \mathrm{H}$ coupling constants. A clear NOE enhancement can be seen from H-6 to $\mathrm{H}-2 \alpha\left(\delta_{\mathrm{H}} 2.49\right)$ assigning these protons to the same face. NOE enhancements can be seen from $\mathrm{H}-2 \alpha$ to the gem-dimethyls and both acetates that assign these groups to the $\alpha$ face as well. $\mathrm{H}-2 \alpha$ appears as a triplet of doublets with a large coupling constant of $10.5 \mathrm{~Hz}$, which assigns it in an anti relationship to both $\mathrm{H}-1$ and $\mathrm{H}-3$, placing these protons axial on the $\beta$ face. This then places the acetylated hydroxyl at C-3 in an equatorial orientation. As the dihydroxylation produces a cis diol the hydroxyl at C-4 must be axial. This is confirmed by a coupling constant between $\mathrm{H}$ 3 and $\mathrm{H}-4$ of $1.7 \mathrm{~Hz}$ which places $\mathrm{H}-4$ in an equatorial orientation to the axial proton at H-3. This is the expected product due to steric factors influencing the initial addition of the osmium tetraoxide to the alkene. Models suggested that the most likely conformation of this oxidation product in solution is again twist-chair (Figure 5.17).

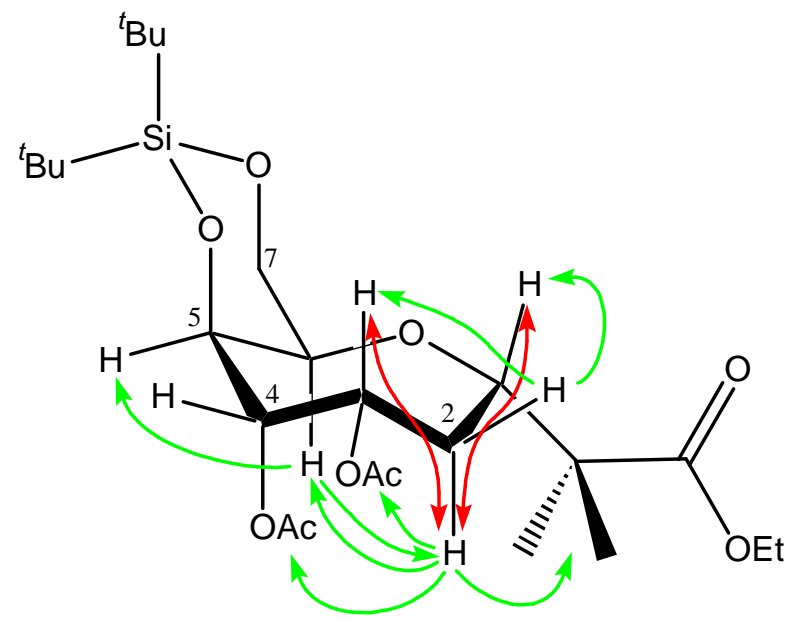

Figure 5.17. Structure of diacetate $\mathbf{5 . 1 2}$ showing NOE enhancements and ${ }^{1} \mathrm{H}$ axial-axial interactions. 


\subsection{Hydroboration of oxepine 3.12}

Several attempts were made to convert the alkene to an alcohol using Browns hydroboration conditions. ${ }^{109,110}$ This reaction adds an alcohol group to the least substituted end of an alkene in an anti-Markovnikov fashion. Addition of a hydroborane species across the alkene is followed by the formation of a borane, which is then converted to a hydroxyl group (Scheme 5.4).

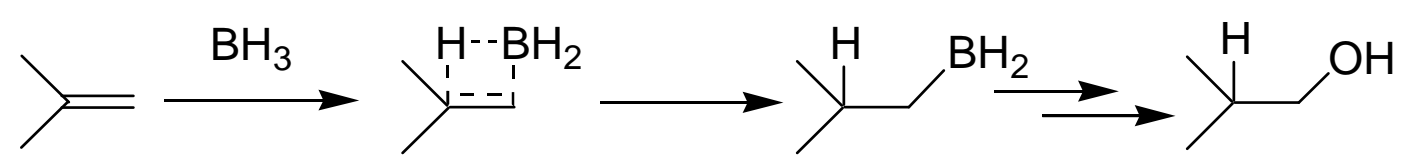

Scheme 5.4. Standard hydroboration reaction showing addition at the least substituted carbon center.

Using more bulky boranes such as 9-BBN increases the selectivity of the addition. Reacting oxepine 3.12 in THF with $9-\mathrm{BBN}$ at $0{ }^{\circ} \mathrm{C}$ followed by addition of $\mathrm{NaOH}$ and $\mathrm{H}_{2} \mathrm{O}_{2}$ then warming to ambient temperature for 12 hours only returned starting material despite repeated attempts. Repeating the reaction substituting $\mathrm{BH}_{3} \cdot \mathrm{DMS}$ as the hydroboration reagent gave one product, $\mathbf{5 . 8}$ in $40 \%$ yield along with $26 \%$ of starting material (entry six, table 5.1). ${ }^{1} \mathrm{H}$ NMR again gave a very congested spectrum with four methine and two oxymethylene signals between $\delta_{\mathrm{H}} 3.9$ and $4.2 \mathrm{ppm}$. It also indicated the expected presence of two unsubstituted methylenes. Acetylation of the alcohol to give 5.13 again dispersed the ${ }^{1} \mathrm{H}$ signals enough to allow assignment of the structure. Connectivity of the ${ }^{1} \mathrm{H}$ spin system was then constructed from a series of COSY correlations from the $\mathrm{C}-1$ oxymethine $\left(\delta_{\mathrm{H}} 3.89, \delta_{\mathrm{C}} 82.4\right)$ to a methylene $\left(\mathrm{C}-2: \delta_{\mathrm{H}} 2.00\right.$ and $\left.1.51, \delta_{\mathrm{C}} 19.7\right)$ to a second methylene $\left(\mathrm{C}-3: \delta_{\mathrm{H}} 1.92, \delta_{\mathrm{C}} 25.5\right)$ to an oxymethine $(\mathrm{C}-4$ : $\left.\delta_{\mathrm{H}} 4.96, \delta_{\mathrm{C}} 73.3\right)$ to a second oxymethine $\left(\mathrm{C}-5: \delta_{\mathrm{H}} 4.10, \delta_{\mathrm{C}} 75.7\right)$. The COSY data reveals that two unsubstituted methylenes are next to each other in the oxepane, 
suggesting that the hydroxylation has occurred at the C-4 position, rather than the expected C-3 position. This is opposite to what is usually generated by the Brown hydroboration, which generates alcohols on the least substituted end of the starting alkene. $^{109,110}$

It is postulated that the boron reagent complexes to the C-5 oxygen and directs the stereo- and regioselectivity of the subsequent alcohol formation overriding the steric conditions that normally drive the regioselectivity. A similar effect was observed previously in the formation of dibromide 5.1. This does not explain why the more bulky 9-BBN failed to give any results, as the steric control would surely be favoured over complexation with the C-5 oxygen. A possible reaction intermediate is shown in Scheme 5.5.

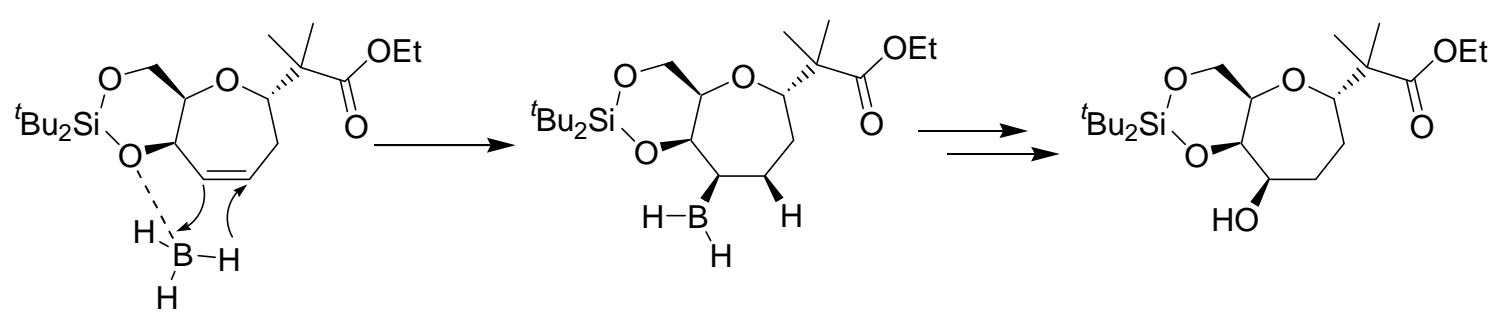

Scheme 5.5. Possible borane intermediate formation leading to the observed alcohol stereochemistry

The conformation and relative stereochemistry around the oxepane ring were established from an analysis of NOESY enhancements and ${ }^{1} \mathrm{H}-{ }^{1} \mathrm{H}$ coupling constants of the acetylated product 5.13. The $\mathrm{H}-1$ proton has a large coupling constant of $12.5 \mathrm{~Hz}$ with $\mathrm{H}-2 \alpha\left(\delta_{\mathrm{H}} 2.00\right)$ placing it axial in an anti relationship. $\mathrm{H}-2 \alpha$ shows clear NOE enhancements to $\mathrm{H}-3 \alpha$ and $\mathrm{H}-4$. This places the acetylated hydroxyl at C-4 on the $\beta$ face which is confirmed by the weak NOE to H-3 $\beta$. These correlations are consistant with the proposed conformation in solution being a twist-chair (Figure 5.1). 


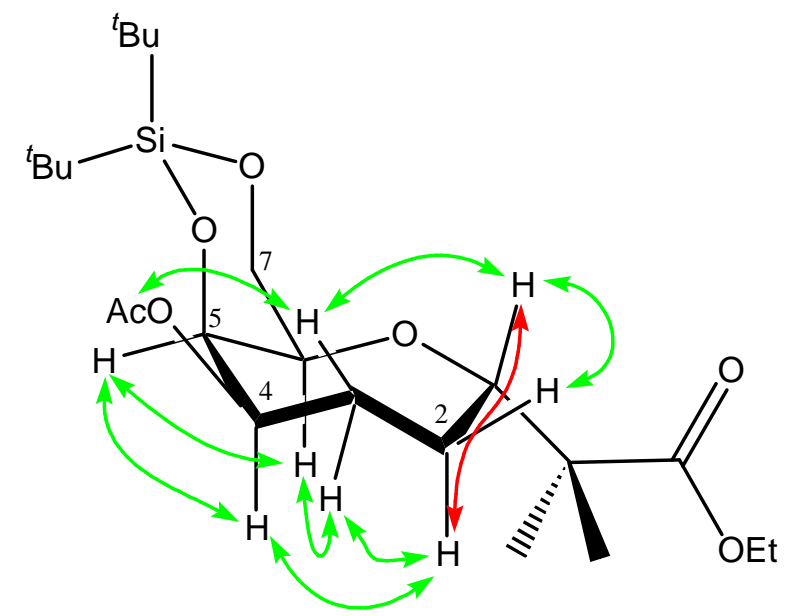

Figure 5.18. Structure of monoacetate $\mathbf{5 . 1 3}$ showing NOESY enhancements and ${ }^{1} \mathrm{H}$ axial-axial interactions.

\subsection{Reduction of the ester of oxepine 3.12}

The chemistry of the ester was investigated with a view towards further expansion of the oxepane from the gem-dimethyl side-chain. Reduction of the ester to the corresponding aldehyde was proposed. It is well precendented that reduction of an ester with DIBAL at $-78{ }^{\circ} \mathrm{C}$ generates an aldehyde selectively. However this method is extremely sensitive and can result in the over reduction of the aldehyde to the corresponding alcohol. ${ }^{111}$ Reaction of oxepine 3.12 with one equivalent of DIBAL in dichloromethane at $-78{ }^{\circ} \mathrm{C}$ gave one alcoholic product, oxepine 5.9 (Figure 5.19) in a $54 \%$ yield along with $23 \%$ recovered starting material (entry seven, table 5.1). ${ }^{1} \mathrm{H}$ and ${ }^{13} \mathrm{C}$ NMR revealed that the desired aldehyde was present in trace amounts, co-eluting with the starting material. All other ${ }^{1} \mathrm{H}$ and ${ }^{13} \mathrm{C}$ data were consistent with oxepine 3.12.<smiles>C[C@@H]1O[C@@H](C(C)(C)CO)CC=C[C@@H]1O[SiH2]O[SiH2]C(C)(C)C</smiles>

Figure 5.19. Alcohol 5.9 from DIBAL reduction of the oxepine ester 3.12. 
This reaction, while unsuccessful, demonstrated the oxepines stability to reducing conditions. A better method to generate the desired aldehyde would be to reduce the ester and oxidise the resulting alcohol $\mathbf{5 . 9}$ over 2 steps as this would result in a higher overall yield.

\subsection{Summary}

The results of the reactions in this chapter are summarised in Table 5.1.

All the reactions in this chapter lead to either the formation of a single product or a mixture of easily separable isomers with the only exception to this being the products of the bromohydrin addition. The reactions all demonstrate high diastereo- and regioselectivity with simple non-selective reagents, and it is relatively easy to predict the stereochemistry of the resulting oxepanes. While the variety of functional groups introduced is not great, there is potential to either displace or alter these to other functionalities. Information was also obtained regarding the conformation of the oxepane in solution. The preferred conformation adopted in solution was that of the twist-chair, with none of the other possible conformers observed. This is in line with other conformations reported for seven membered rings. ${ }^{98}$ These results demonstrate the synthetic utility of the oxepine moiety as a synthon. 
Table 5.1. Results of elaborations of oxepine 3.12.

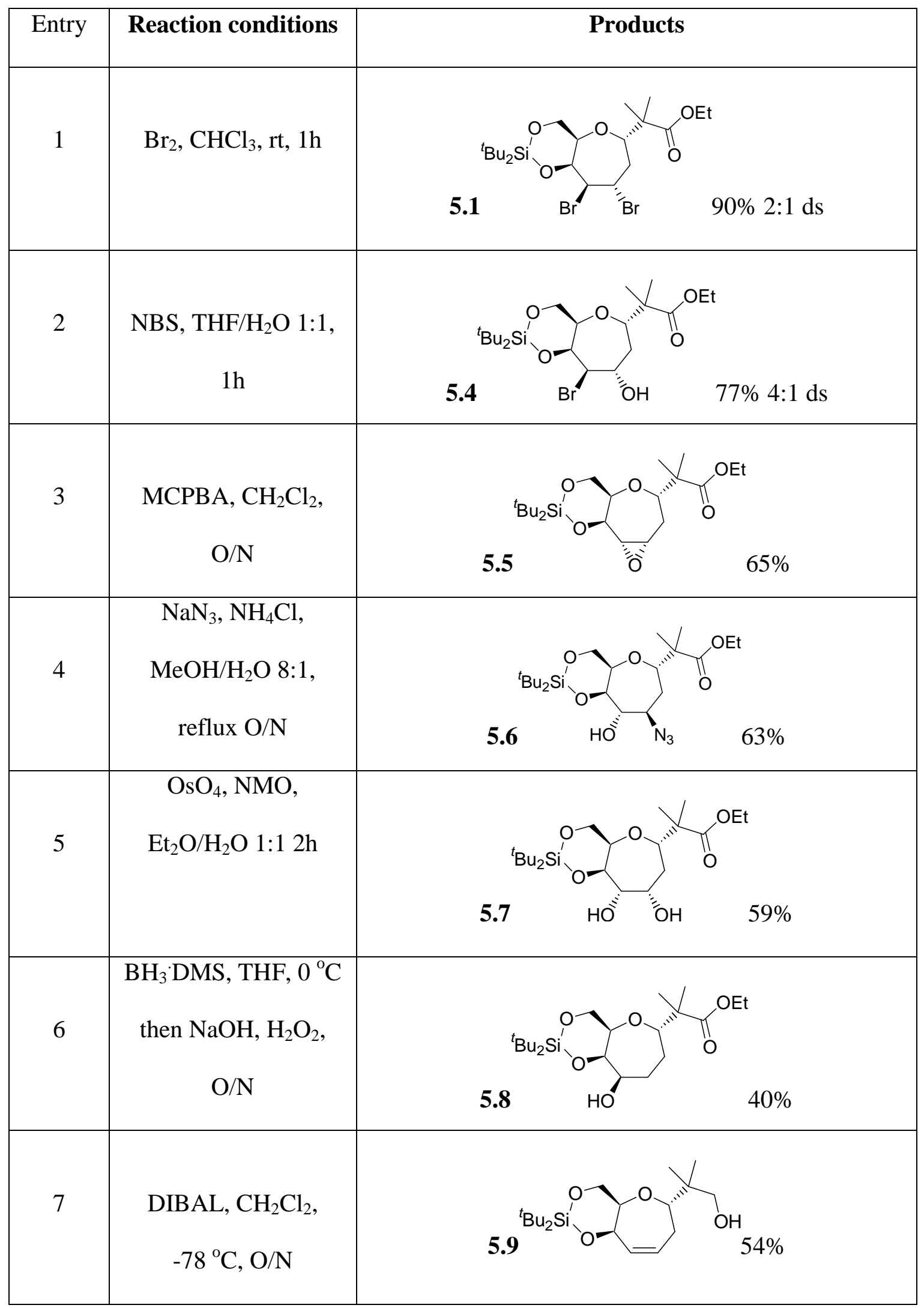




\section{Chapter 6: Synthesis of a natural compound using the developed ring expansion methodology}

'If you're looking to get to there, I wouldn't be starting from here'

As a progression from the investigation of the ring expansions of cyclopropanated glycals, attention turned to the application of the methodology to the synthesis of a naturally occurring compound.

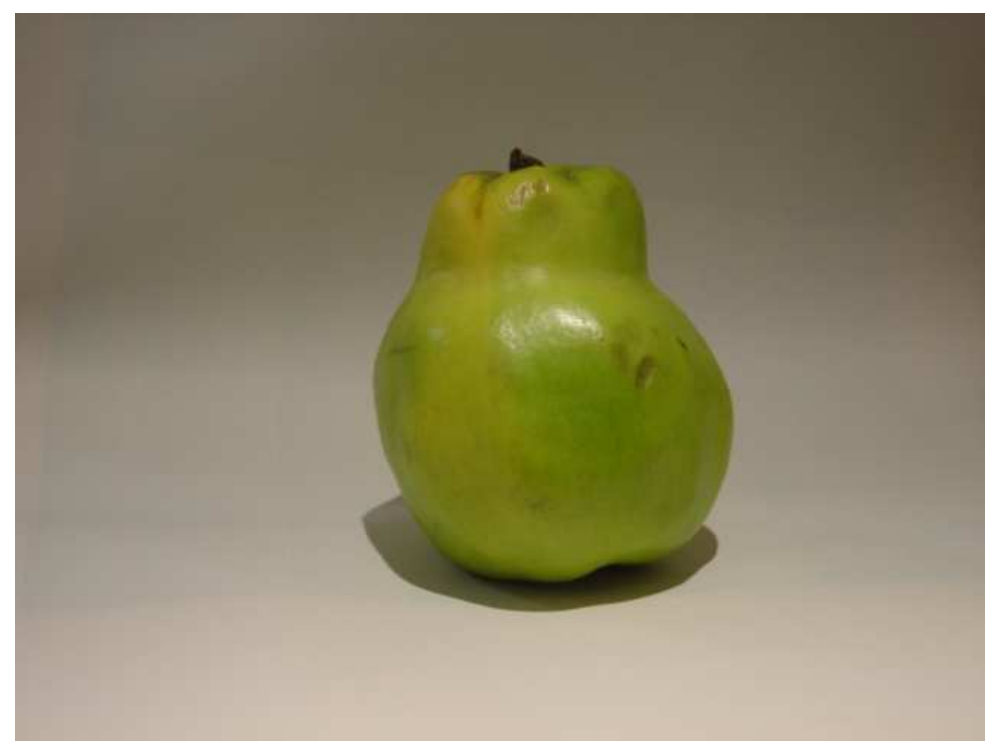

Figure 6.1. Cydonia obolonga.

The fruit of the quince Cydonia oblonga (Figure 6.1) has a very powerful and distinct flavour that is used in marmalade, candied fruits, sweets and brandy. This flavour is the result of a large number of both saturated and unsaturated esters and ethers present in trace amounts in the fruit. During the course of an in-depth investigation of the aromatic components several $\mathrm{C} 12$ compounds with a seven membered ether ring were identified from a sample of Swiss quince brandy. ${ }^{112}$ Two of these are shown in Figure 6.2 . 

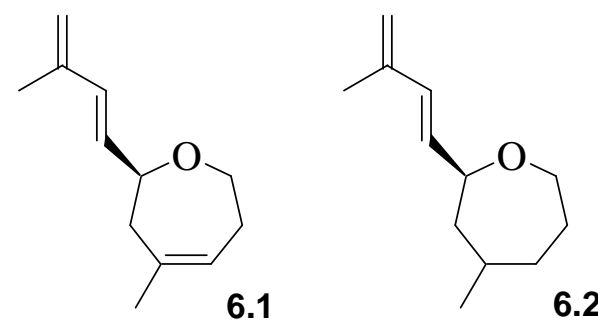

Figure 6.2. Seven membered ring ethers isolated from quince brandy.

The compounds were identified on the basis of mass spectroscopy and ${ }^{1} \mathrm{H}$ NMR data. The proposed structures were confirmed by a total synthesis of the compounds published in 1991 by Escher and Niclass. ${ }^{113}$ The synthetic scheme involved building up the carbon chain followed by $\mathrm{C}-\mathrm{O}$ bond formation as the final step and gave oxepine $\mathbf{6 . 1}$ in nine steps and $2 \%$ overall yield as a racemic mixture (Scheme 6.1).
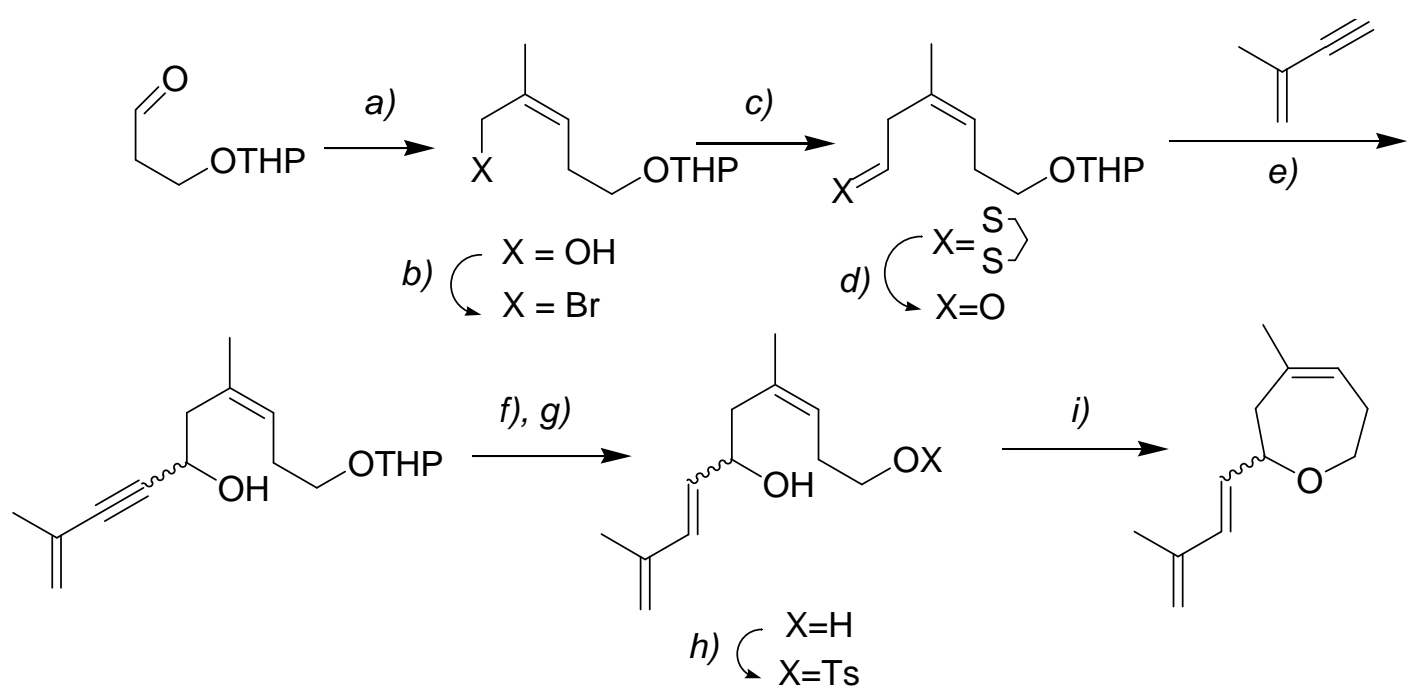

a) $\mathrm{PPh}_{3}=\mathrm{CHMe}, \mathrm{BuLi}, \mathrm{CH}_{2} \mathrm{O}, 58 \%$. b) $\mathrm{PBr}_{3}$, py, hexane, $-5{ }^{\circ} \mathrm{C}, 42 \%$. c) 2-Lithiodithiane, 65\%. d) $\mathrm{MeI}$, $\mathrm{CaCO}_{3}, \mathrm{MeCN} / \mathrm{H}_{2} \mathrm{O}, 87 \%$. e) BuLi, monoglyme, $0{ }^{\circ} \mathrm{C}, 99 \%$. f) LAH, THF, reflux. $g$ ) $10 \%$ aq. $\mathrm{HCl}$, $\mathrm{MeOH}, 60 \% / 2$ steps. h) 1.1 eq. TsCl, $\mathrm{Py}, 0{ }^{\circ} \mathrm{C}, 62 \%$. i) 3.0 eq. $\mathrm{NaH}, 1$ eq. DMPU, monoglyme, $40 \%$.

Scheme 6.1. Escher and Niclass' synthesis of oxepane 6.1.

These compounds were chosen as the synthetic target because they had relatively simple structures which contained features similar to our previously synthesised 
oxepines. The previous synthesis had also been low yielding and racemic whereas our strategy would be stereoselective.

\subsection{Initial synthetic plan starting from cyclopropane 3.3.}

It was initially envisioned that the formation of the main oxepine ring would utilise the general procedure laid out in Section 3.1 in which protection of the galactal ring with the di-tert-butylsilyl moiety would be followed by cyclopropanation. At this point it was planned to insert the methyl group into the ring corresponding to the target molecule that has a methyl substituent at C-4. By harnessing the deoxygenation discovered in the course of the expansions of cyclopropane 3.4 (see Scheme 4.6) it would then prove possible to remove the hydroxyl at C-5 during the course of the ring expansion step. A one pot deprotection and oxidation would be followed by a HornerWadsworth-Emmons olefination to yield the desired product (Scheme 6.2).
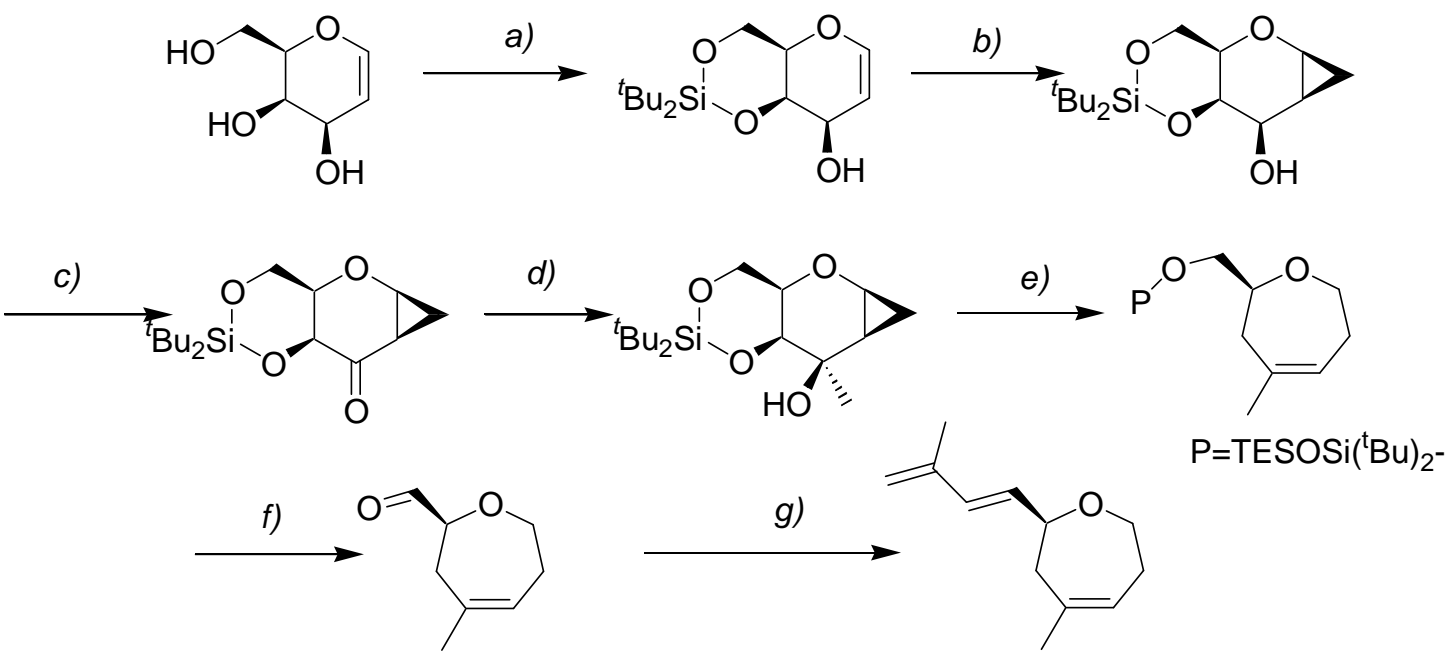
a) Silyl ether formation.
b) Simmons-Smith cyclopropanation.
c) Swern oxidation.
d) Grignard methylation. e) Ring expansion. $f$ ) Oxidative desilylation. $g$ ) Horner-Wadsworth-Emmons.

Scheme 6.2. Projected reaction scheme for the total synthesis of quince oxepane. 
At this point the method of cyclopropanation was altered. This was brought about by problems with the local supply of $\mathrm{Et}_{2} \mathrm{Zn}$ in hexanes used for the Furukawa modification of the Simmons-Smith methodology. Difficulties were experienced with shipping the chemical into the country from overseas, and also with its purity and longevity once it had arrived in the laboratory. It was decided to perform a trial reaction with galactal $\mathbf{3 . 1}$ using the method of Shank and Shechter ${ }^{114}$ with a Zinc-Copper couple. ${ }^{115}$ This gave the desired cyclopropane $\mathbf{3 . 3}$ in almost identical yield and selectivity to that experienced with the use of $\mathrm{Et}_{2} \mathrm{Zn}$. This method was then used for the remainder of the project.
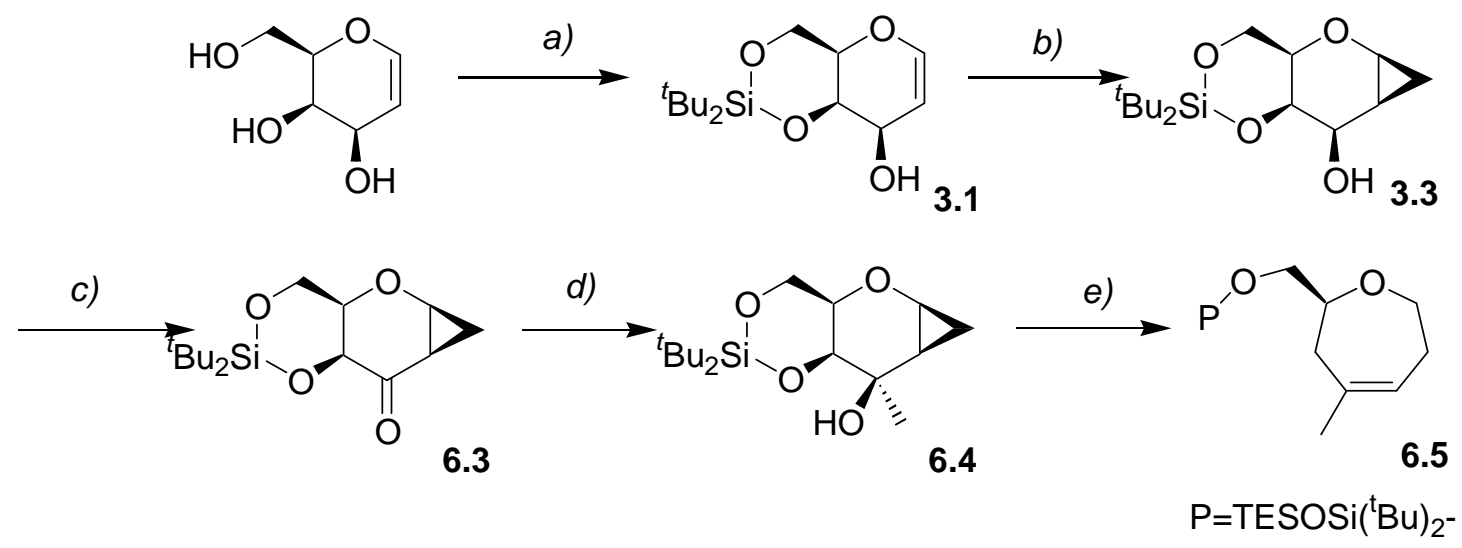

a) Di-tert-butylsilyl bistriflate, py, DMF, $-40{ }^{\circ} \mathrm{C}, 65 \%$. b) $\mathrm{Cu}-\mathrm{Zn}$ couple, $\mathrm{CH}_{2} \mathrm{I}_{2}, \mathrm{Et}_{2} \mathrm{O}, 73 \%$. c) Swern oxidation, $94 \%$. d) $\mathrm{MeMgBr}, \mathrm{Et}_{2} \mathrm{O}, 0{ }^{\circ} \mathrm{C}, 86 \%$, see text. e) TESH, TMSOTf, MeCN.

Scheme 6.3. Reaction scheme for the formation of oxepine $\mathbf{6 . 5}$.

The initial synthetic route is shown in Scheme 6.3. Cyclopropane 3.3 was synthesised as described previously (Section 3.1) and was then subjected to a Swern oxidation ${ }^{116}$ to give ketone 6.3 in a $94 \%$ yield. Treatment of ketone 6.3 with $\mathrm{MeMgBr}$ in $\mathrm{Et}_{2} \mathrm{O}$ gave cyclopropane 6.4 in an $86 \%$ yield. However it proved impossible to acetylate the tertiary alcohol at C-3 in a usable yield. Small amounts of the acetylated product (less that $5 \%$ ) were isolated from the protection reaction. It is postulated that the steric crowding of the $\beta$ face makes the introduction of the acetyl group to the C-3 alcohol unfavourable. 
Despite the lack of a leaving group at C-3, cyclopropane 6.4 was then subjected to the ring expansion conditions. It was thought that a suitable leaving partner would be formed in situ. Ring expansion followed by the standard aqueous workup gave a very low isolated yield of the desired oxepine 6.5. This had also been observed at the analogous step by a previous worker utilising the glucal skeleton as the starting material instead of galactal but in an analogous reaction scheme. ${ }^{117}$ Examination of the ${ }^{1} \mathrm{H}$ and ${ }^{13} \mathrm{C}$ NMR data of cyclopropane 6.4 revealed that during the Grignard addition the silyl protecting group had cleaved from the hydroxyl at C-4, resulting in a di-tert-butyl methyl protecting group at the C-6 position (Scheme 6.4). This was confirmed by the presence of a methyl signal at $\delta_{\mathrm{H}} 0.10$ in the ${ }^{1} \mathrm{H}$ NMR, indicative of a methyl group attached to a silicon atom.

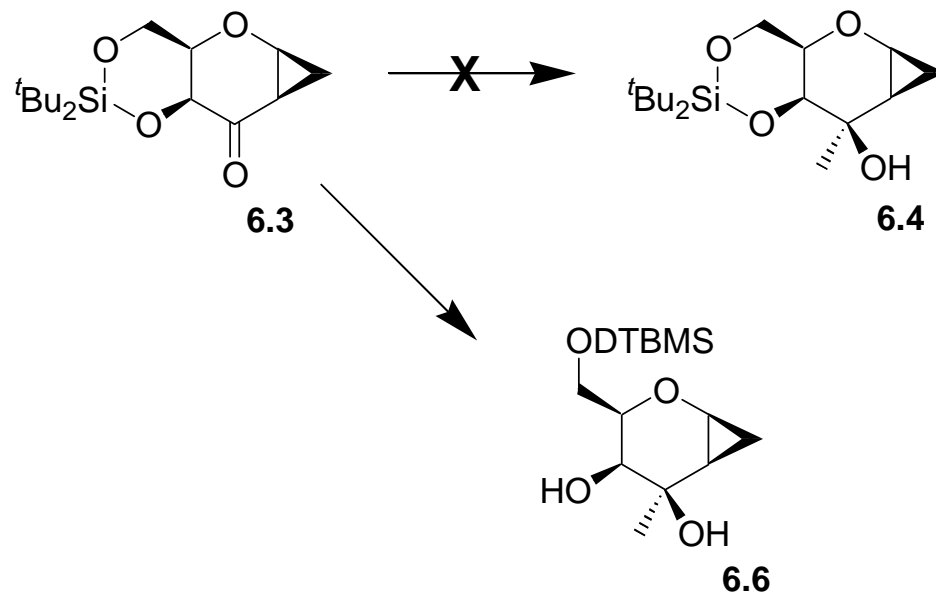

Scheme 6.4. Mono deprotection of 6.3 to give diol 6.6.

Treatment with $\mathrm{MeMgBr}$ is known to convert acetonides to a tert-butyl group selectively deprotecting a secondary hydroxyl over a primary hydroxyl. ${ }^{118}$ However it has not been previously reported in the case of di-tert-butylsilyl group. Cyclopropane 6.6 then undergoes deprotection during the ring expansion step as has been previously observed for non-tethered silyl protecting groups and the diol is then lost in the aqueous phase during workup. In subsequent ring expansions the reaction mixture was 
neutralised with triethylamine and then filtered through a silica plug in order to avoid these aqueous conditions. Elution with hexanes removed the residual TES- and DTBMS- derived by-products followed by elution with EtOAc to give the oxepine diol 6.7 in a $60 \%$ yield (Scheme 6.5).

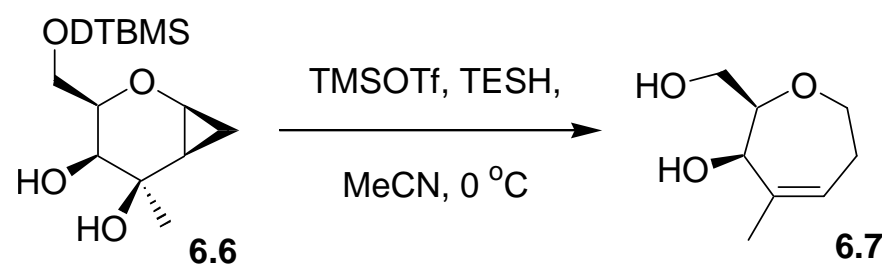

Scheme 6.5. Formation of oxepine diol 6.7.

Attempts were made to oxidise $\mathbf{6 . 7}$ to the desired aldehyde. Several different oxidation methods, including Swern ${ }^{116}, \mathrm{PCC}^{119}$ and Dess-Martin periodane ${ }^{120}$, were tried. Small amounts of aldehydes were observed in the ${ }^{1} \mathrm{H}$ NMR from these reactions, but these proved impossible to purify by flash chromatography on silica gel and were not pursued further due to the unsatisfactory yields. It was postulated that the presence of the hydroxyl group at C-5 leads to hydrogen bonding with the primary alcohol at C-7, thereby inhibiting oxidation (Figure 6.3). Aqueous work ups used for the oxidation then removed the unreacted diol 6.7. Thus no starting material was recovered from any of these reactions.

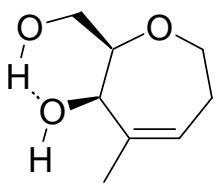

Figure 6.3. Possible $\mathrm{H}$ bonding in diol 6.7 preventing oxidation of primary alcohol. 


\subsection{Revised synthetic plan starting from cyclopropane 1.61}

In an attempt to circumvent the observed problems with the initial synthetic route it was decided to synthesise the oxepine using glucal as a starting material, as had originally been attempted by a co-worker. ${ }^{117}$ It was thought that it should be possible to acetylate the cyclopropanated glucal alcohol as there would be little or no steric interference due to the planar nature of the molecule. This would then lead to retention of the silyl protecting group and an easier purification of the ring expansion products. However it would also introduce extra steps into the synthesis as the alcohol on the oxepine ring would have to be removed separately, for example by Barton-McCombie deoxygenation (Scheme 6.6). ${ }^{121}$
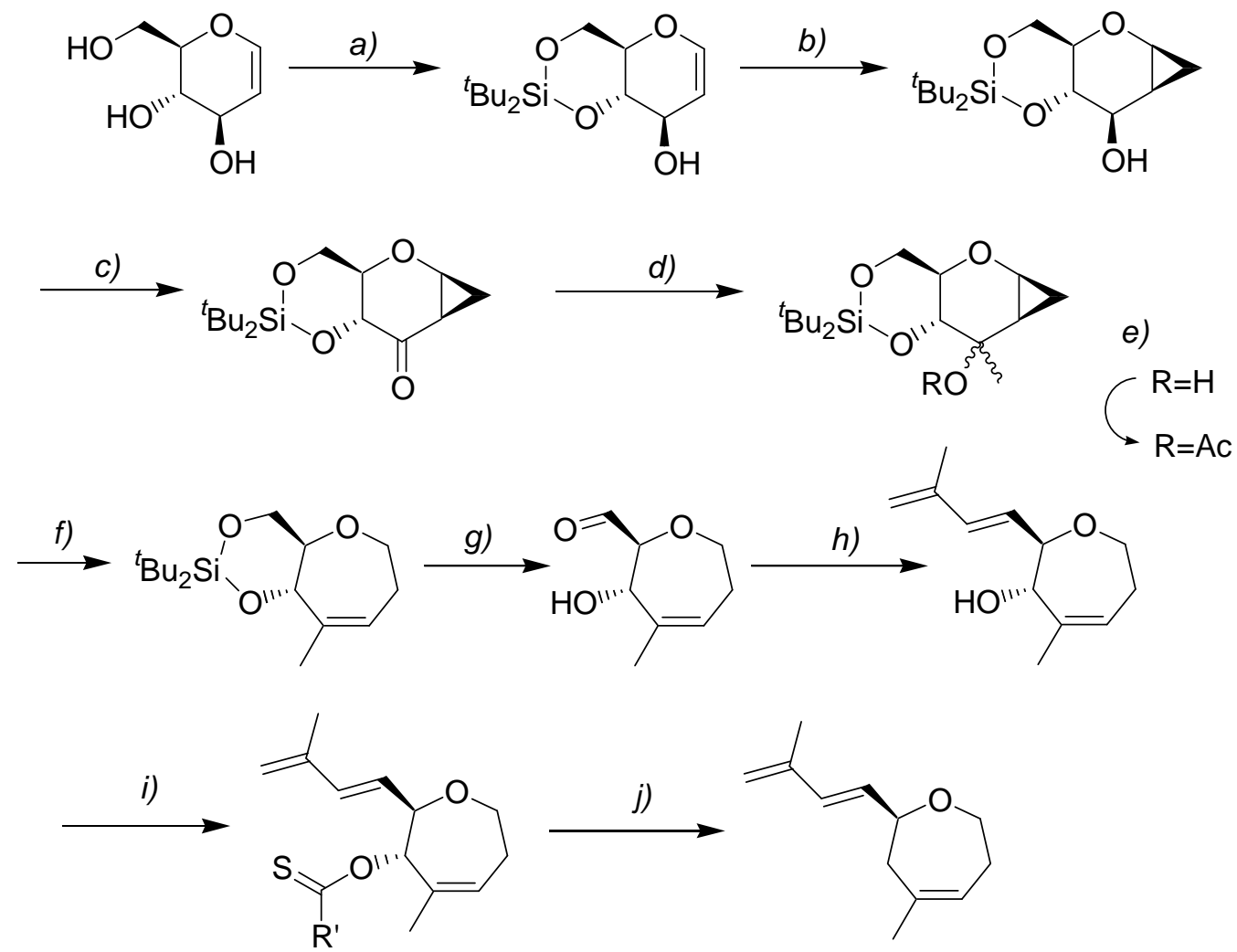

a) Silyl ether formation. b) Simmons-Smith cyclopropanation. c) Swern oxidation. d) Grignard methylation. e) Acetylation. f) Ring expansion. g) Oxidative desylation. h) Horner-WadsworthEmmons reaction. i) Xanthate formation. $j$ ) Barton-McCombie deoxygenation.

Scheme 6.6. Revised synthetic scheme using glucal as a starting point. 
The cyclopropane 1.61 was synthesised using Hoberg's published method. ${ }^{70}$ This compound was then oxidised under Swern conditions ${ }^{116}$ to give ketone $\mathbf{3 . 2 0}$ as before. The Grignard addition to ketone 3.20 was performed with freshly generated MeMgI to give cyclopropane $\mathbf{6 . 8}$ in a $99 \%$ yield. This reaction gave only one isomer by ${ }^{1} \mathrm{H}$ NMR, which was not expected. Examination of the ketone 3.20 demonstrates that Grignard attack is more likely from the sterically less hindered $\alpha$ face (Scheme 6.7).

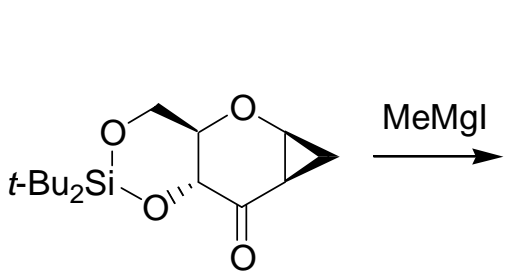

3.20

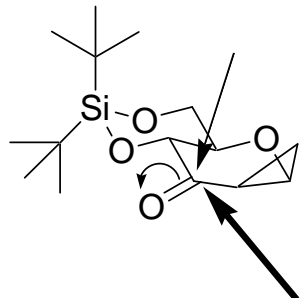

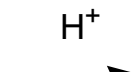

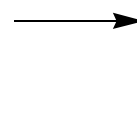

Scheme 6.7. Grignard addition to ketone 3.20 showing preferred route of attack.

However, it again proved impossible to actetylate the C-3 hydroxyl using standard conditions. This suggested that the previous theory of steric hindrance was incorrect and that it is the inherent unreactivity of the tertiary alcohol that is preventing the acetylation. It was again decided to proceed with the ring expansion step with formation of a suitable leaving group in situ as previously demonstrated in the expansion of cyclopropane 6.6. No products could be isolated from the ring expansion, and no starting materials were recovered. Similar results had been observed with cyclopropane 3.4 when no nucleophile was present in the ring expansion reaction. This synthetic route was therefore discarded.

\subsection{Modification of the initial synthetic route.}

Attention then returned to the initial strategy using the galactal starting material. To ameliorate the problems previously experienced in the oxidation step it was decided to 
use a recently reported one pot method for the oxidation of the oxepine primary alcohol followed by a Wittig reaction to attach the diene side-chain. Vatele reported generating aldehydes from primary alcohols with TEMPO and BAIB and then converting them to a trans-alkene in situ by addition of an activated phosphine at $0{ }^{\circ} \mathrm{C} .{ }^{122} \mathrm{~A}$ modification of reaction scheme 6.2 was then proposed. This would see the one pot oxidation/Wittig followed by another Wittig reaction to complete the formation of the sidechain, and the final removal of the hydroxyl group at C-5 with a Barton-McCombie deoxygenation (Scheme 6.8). ${ }^{121}$
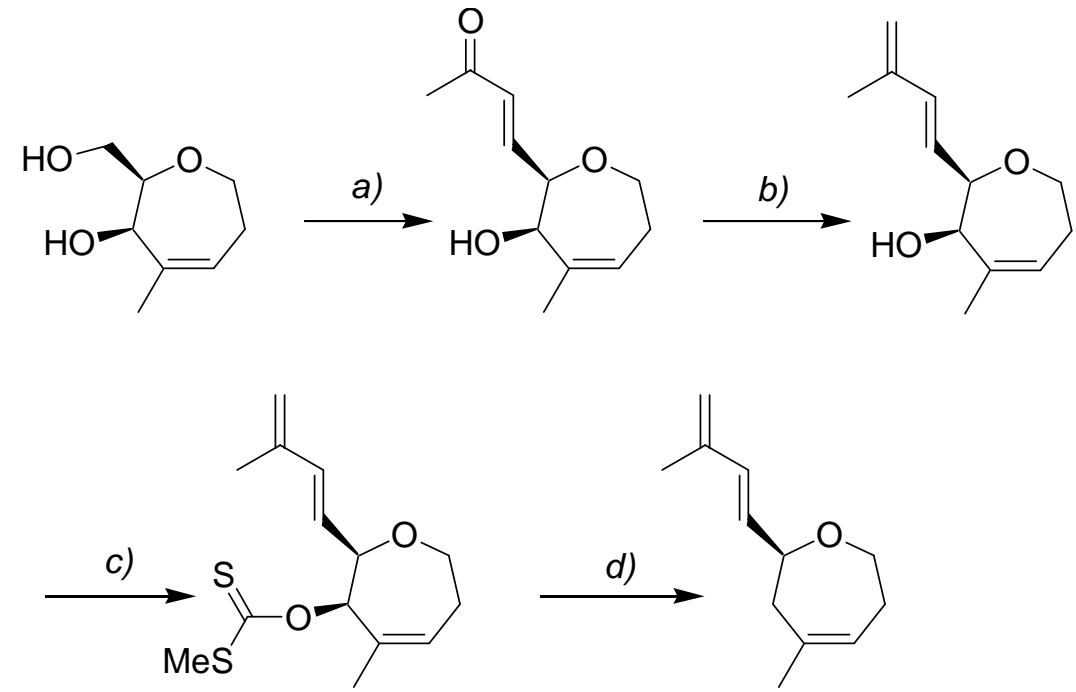

a) Oxidation/Wittig. b) Wittig. c) Xanthate formation. $d$ ) Barton-McCombie deoxygenation.

Scheme 6.8. Reaction scheme for installation of the side chain.

In order to trial the methodology, Vatele's one pot oxidation/Wittig was applied to diol 6.7 using a similar stabilised methyl ester containing phosphoranylidene that was on hand. The desired product 6.9 was isolated in $27 \%$ yield (Scheme 6.9). Also isolated was a similar by-product in $31 \%$ yield. 


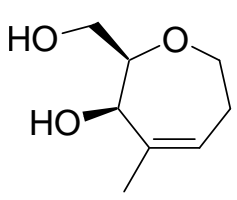

6.7
1) $T E M P O / B A I B$, $\mathrm{CH}_{2} \mathrm{Cl}_{2}, \mathrm{RT}$

2) Wittig

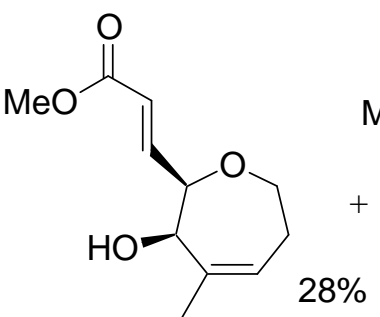

6.9<smiles>COC(=O)/C=C/[C@H]1OCCC=C1C</smiles>

6.10

Scheme 6.9. Products from the oxidation/wittig reaction.

Initial ${ }^{1} \mathrm{H}$ NMR of the by-product gave promising spectra with signals indicating the presence of a new alkene $\left(\delta_{\mathrm{H}}=6.95\right.$ and $\left.\delta_{\mathrm{H}}=6.06\right)$ with a large coupling constant of 15.5 $\mathrm{Hz}$ which suggested a trans alkene was present. However, ${ }^{13} \mathrm{C}$ NMR revealed that there were only 10 carbons present in the molecule vs. the expected 11. HSQC and HMBC experiments proved that the alcohol and its adjacent carbon were missing from the oxepine ring. To explain these observations structure $\mathbf{6 . 1 0}$ was proposed and confirmed by HRMS results.

The mechanism for the formation of this compound was initially unclear. It was proposed that aldehyde formed from the diol 6.7 underwent further oxidation to the acid, and then a radical decarboxylation. A combination of oxonium formation and interception by the C-5 hydroxyl could be responsible for the ring contraction. The resulting aldehyde would then undergo a Wittig reaction with the methyl ester containing phosphoranylidene in situ to form $\mathbf{6 . 1 0}$ (Scheme 6.10).

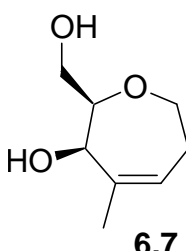<smiles>CC1=CCCOC(C(=O)O)C1O</smiles>

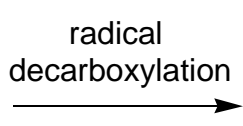<smiles>CC1=CCCOC1C=O</smiles><smiles>COC(=O)/C=C/[C@H]1OCCC=C1C</smiles>

6.7

Scheme 6.10. Possible mechanism formation of the pyrenyl side product.

The reaction was then repeated at $0{ }^{\circ} \mathrm{C}$ under an inert atmosphere with the intention of minimising over oxidation to the carboxylic acid. The correct phosphoranylidene with 
the methyl group was now avaliable and was substituted for the previously used methyl ester containing phosphoranylidene at this point. Reaction of oxepine 6.7 with TEMPO and diacetoxyiodobenzene at $0{ }^{\circ} \mathrm{C}$ followed by addition of the Wittig ylide gave $\mathbf{6 . 1 1}$ in a $40 \%$ yield (Scheme 6.11).

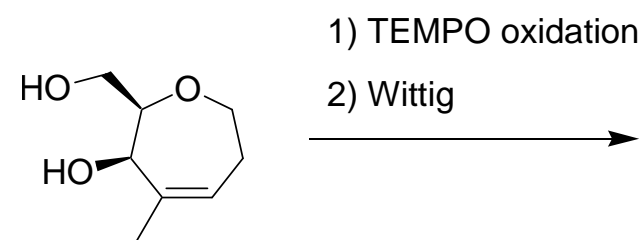

6.7

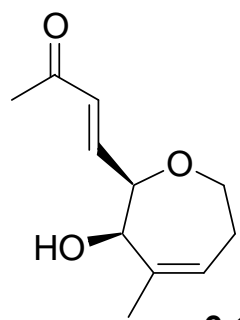

6.11

Scheme 6.11. Formation of oxepine 6.11.

No pyrene by-product identified in the previous reaction could be isolated from this reaction mixture. It is possible that while the pyrene compound is indeed formed in the course of the reaction, its lower boiling point compared to $\mathbf{6 . 1 1}$ precluded isolation as it may have been evaporated along with the solvent during concentration.

Continuing on the synthetic strategy shown in Scheme 6.8, a Wittig reaction was then attempted to convert the carbonyl group on the side chain to the corresponding alkene. Reaction of 6.11 with $\mathrm{nBuLi}$ and $\mathrm{MePPh}_{3} \mathrm{Br}$ in $\mathrm{THF}$ failed to generate the expected product. Isolated from the reaction were four compounds as two pairs of diastereoisomers. One pair was provisionally identified as an n-butyl addition product at the carbonyl to give compound $\mathbf{6 . 1 2}$ (Figure 6.4), while the other had undergone rearrangement that had destroyed the stereochemistry at the C-5 and C-6 positions followed by addition of an $n$-butyl group. Further identification of these products was not pursued. An attempt to use Tebbe's reagent was made to generate the olefin. ${ }^{64}$ This reaction was also unsuccessful and none of the desired product was isolated. 


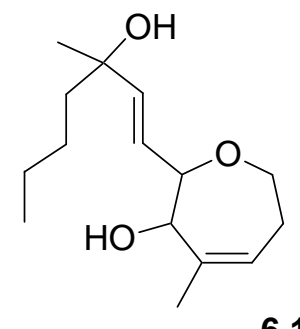

6.12

Figure 6.4. Product 6.12 formed from Wittig reaction with 6.11.

It was then decided to try to form a xanthate on the C-5 hydroxyl of oxepine $\mathbf{6 . 1 1}$ with the carbonyl present. It was intended that this could be used as a protecting group and then removed as the last step in the synthesis. Reaction of oxepine $\mathbf{6 . 1 1}$ with $\mathrm{NaH}, \mathrm{CS}_{2}$ and MeI led to an unidentifiable mixture of low polarity compounds that could not be separated. The reaction conditions were obviously too harsh for the functional groups present.

At this point in the synthesis there was some question as to the presence of a free hydroxyl at C-5 of oxepine $\mathbf{6 . 1 1}$, as a corresponding peak in the ${ }^{1} \mathrm{H}$ NMR spectrum for the $\mathrm{OH}$ proton could not be identified (although this is not uncommon for exchangeable protons in ${ }^{1} \mathrm{H}$ NMR). The presence of the C-5 hydroxyl was demonstrated by acetylation of oxepine $\mathbf{6 . 1 1}$ to give the protected oxepine $\mathbf{6 . 1 3}$ in a $67 \%$ yield (Scheme $6.12)$.

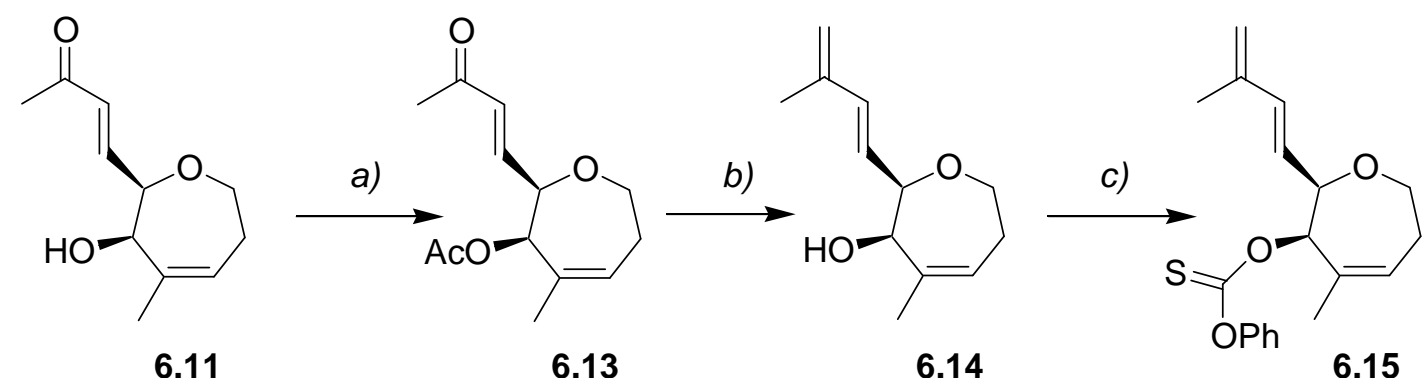

a) $\mathrm{Ac}_{2} \mathrm{O}$, py, DMAP, $\mathrm{CH}_{2} \mathrm{Cl}_{2}, 67 \%$. b) nBuLi, $\mathrm{MePPh} 3 \mathrm{Br}, \mathrm{THF}, 95 \%$. c) Thiophenylorthoformate, imidazole, $\mathrm{CH}_{2} \mathrm{Cl}_{2}, 70 \%$.

Scheme 6.12. Formation of sidechain diene and xanthate 6.15. 
Reaction of 6.13 under Wittig conditions with methylene triphenylphosphonium bromide gave the expected diene $\mathbf{6 . 1 4}$ in a $95 \%$ yield and also resulted in the deprotection of the C-5 hydroxyl in the same reaction pot. With the sidechain diene 6.14 in hand formation of a xanthate under mild conditions with phenyl chlorothionoformate and imidazole proceeded smoothly to form the phenylthioformate 6.15 in a $70 \%$ yield.

The last step of the synthesis was Barton-McCombie deoxygenation which would provide the target oxepine $\mathbf{6 . 1}$ as a single compound. Treatment of phenylthioformate 6.15 with $\mathrm{nBu}_{3} \mathrm{SnH}$ and $\mathrm{AIBN}$ in refluxing toluene yielded an indecipherable mix of products. The target oxepine 6.1 could not be identified by ${ }^{1} \mathrm{H}$ NMR in the crude mixture of compounds. This in itself was not entirely unexpected as the BartonMcCombie deoxygenation is a radical process and it was possible that the radical formed would rearrange around the ring. The desired intermediate required for the formation of the target oxepine $\mathbf{6 . 1}$ may not be the most stable radical intermediate (Scheme 6.13). The deoxygenation reaction could lead to two possible radical centres, however this does not explain the complex mixture of reaction products observed.

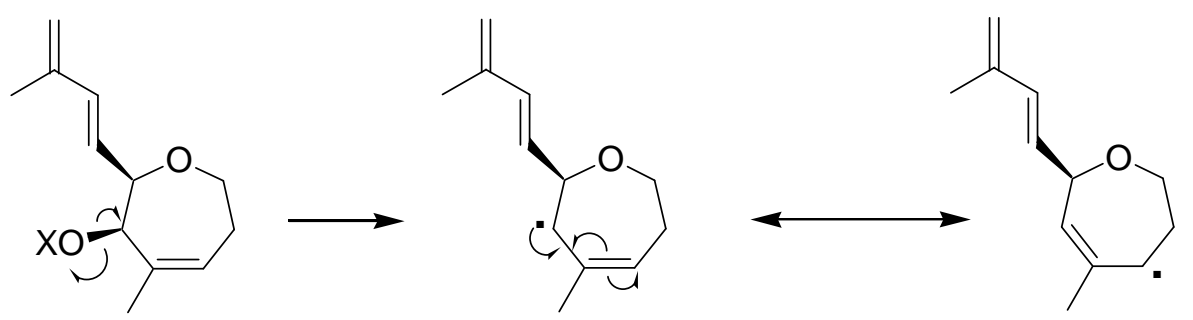

Scheme 6.13. Possible radical isomers formed during Barton-McCombie deoxygenation.

Thus while these proposals explained many of the observations, a few questions remained unanswered. This suggested that an alternative explanation was required. 


\subsection{Unexpected rearrangements during formation of diol 6.7}

\subsubsection{Identification of unexpected products}

At this point a by-product isolated from the products of the ring expansion reaction (Scheme 6.5) provided the first evidence as to what had actually happened during the reaction. An NMR sample of diol 6.7 in $\mathrm{CDCl}_{3}$ was washed back into a flask with acetone and left on the bench. It was subsequently discovered that the sample had undergone a reaction in the flask presumably catalysed by the slightly acidic $\mathrm{CDCl}_{3}$, to give the acetonide protected diol 6.16. During the NMR investigation to identify this product it was noticed that the acetonide carbon was present at $\delta_{\mathrm{C}} 110$ in the ${ }^{13} \mathrm{C}$ spectrum. A literature search revealed that the carbon chemical shift on an acetonide carbon is diagnostic for the size of the ring it is part of. For six membered rings the chemical shift is $100 \mathrm{ppm}$ and for five membered rings it is $110 \mathrm{ppm} .^{123}$ Additional analysis using HMBC NMR experiments revealed that the structure of the diol was actually a six membered ring with an exocyclic diol (Figure 6.5).

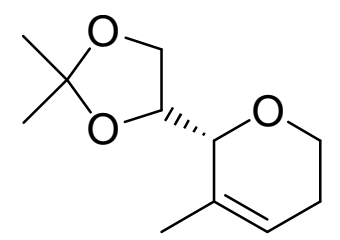

Figure 6.5. Structure of cyclohexyl pyrene 6.16.

This had been missed over nearly three years of research, as HMBC experiments were not available in our laboratories routinely at the inception of this part of the project. The available spectroscopic data at the time was in agreement with other work in the 
formation of oxepines using this method, consequently the formation of a six membered ring had not been considered as a possible reaction product.

Whilst the discovery of the 'cuckoo in the nest' was a surprise, it made sense of many earlier results, which had required convoluted explanations. The reassignment of these structures revealed a far more reasonable path and also explained the failure of the final deoxygenation step. The revised reaction route is shown in Scheme 6.14.
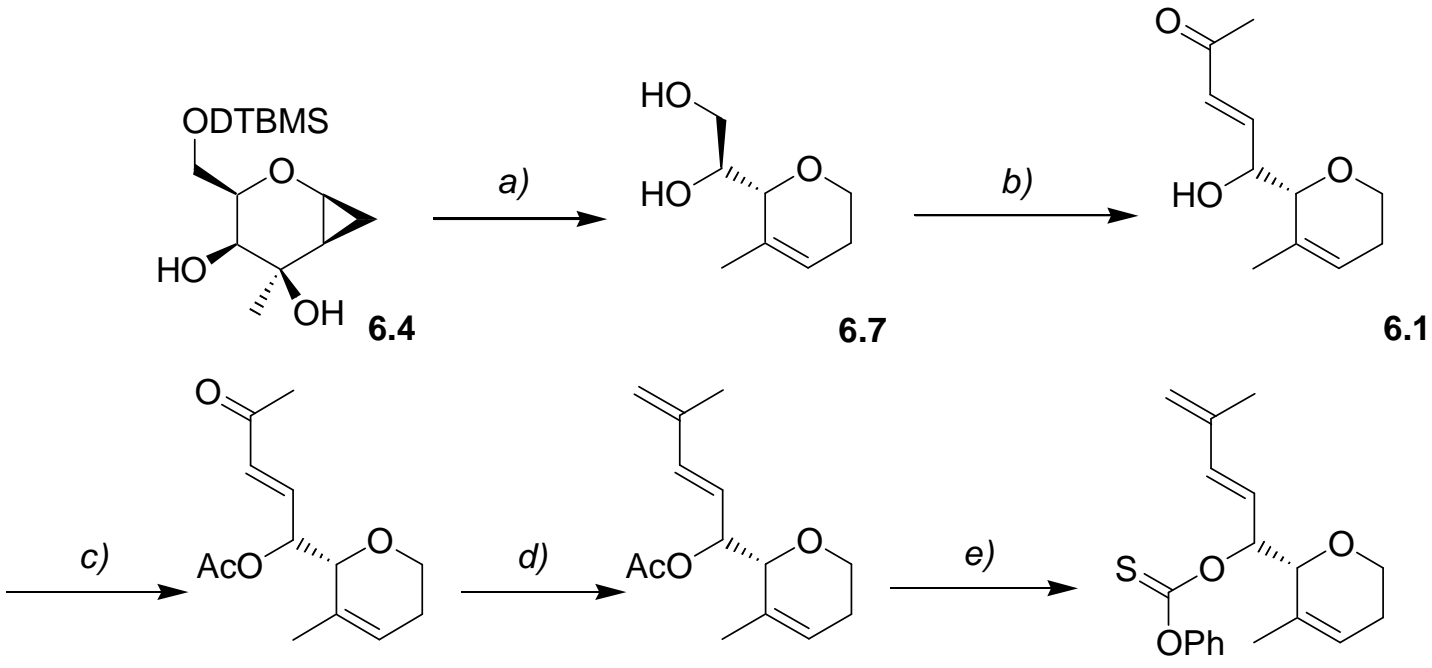

6.13

6.14

6.15

a) TESH, TMSOTf, MeCN, 65\%. b) i) TEMPO, BAIB, $\mathrm{CH}_{2} \mathrm{Cl}_{2}$. ii) $\mathrm{Ph}_{3} \mathrm{PCHCOCH}_{3}, 40 \% . c$ ) $\mathrm{Ac}_{2} \mathrm{O}$, py, DMAP, $\mathrm{CH}_{2} \mathrm{Cl}_{2}, 67 \%$. d) $n \mathrm{BuLi}, \mathrm{MePPh}_{3} \mathrm{Br}$, THF, 95\%. e) phenylchlorothionoformate, imidazole, $\mathrm{CH}_{2} \mathrm{Cl}_{2}, 70 \%$

Scheme 6.14. Revised synthetic scheme for formation of the sidechain.

The Barton-McCombie reaction on $\mathbf{6 . 1 5}$ would have formed a radical center on the sidechain next to a conjugated alkene system, explaining the multitude of inseperable nonpolar products observed.

This also explains the formation of the pyrenyl $\mathbf{6 . 1 0}$ during the one pot oxidation/Wittig reaction shown in Scheme 6.9. A literature search revealed that 1,2-diols when treated 
with an aryl iodosoacetate in the presence of acetic acid cleave to give the corresponding aldehydes. ${ }^{124}$ In this case the acetic acid was formed from reaction of the iodosoacetate with small amounts of water present in the $\mathrm{CH}_{2} \mathrm{Cl}_{2}(\mathrm{Scheme} 6.15)$.

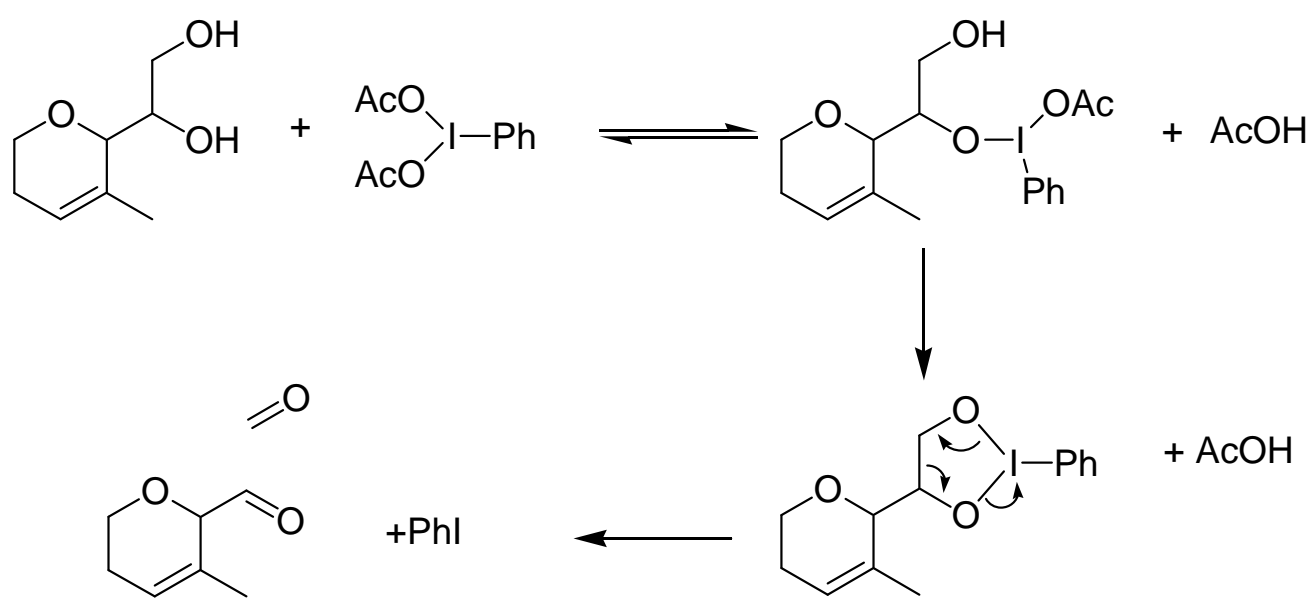

Scheme 6.15. Diol clevage by BAIB.

Thus the mixture of products from the reaction of diol 6.7 in Scheme 6.9 can be explained by the two possible pathways presented in Scheme 6.16.

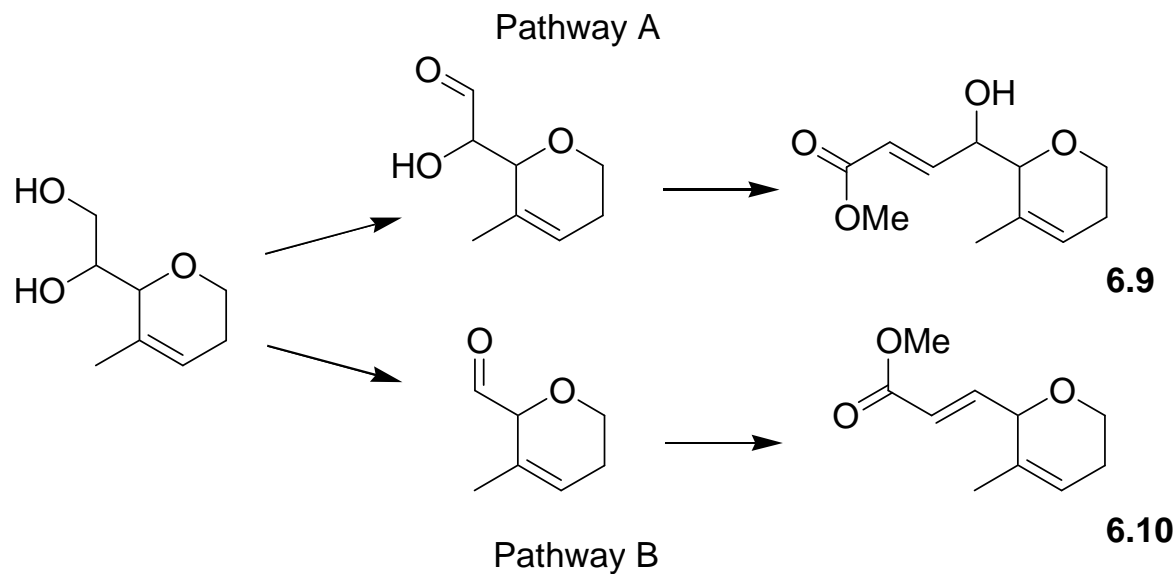

Scheme 6.16. Possible reaction paths for formation of the products 6.9 and 6.10 in scheme 6.9.

In pathway A the primary alcohol of the diol is oxidised to the aldehyde which then reacts with the added Wittig ylide to give the desired product 6.9. In pathway B the diol undergoes oxidative clevage by the BAIB to give an aldehyde which then reacts with 
the Wittig ylide giving the product 6.10. However, products arising from pathway B are not observed in other reactions.

A literature search revealed that the compounds synthesised were previously unknown, starting with the diol 6.7. All compounds exhibited odours consistent with those reported previously for the structurally similar oxepines ${ }^{112,113}$ (namely a slight floral odour). Their flavours were not determined!

\subsubsection{Analysis of the mechanism of ring expansion of cyclopropane 6.6}

Attention now shifted to the mechanism of cyclopropane opening leading from cyclopropane $\mathbf{6 . 6}$ to diol 6.7. The formation of the six membered ring during the cyclopropane opening by TESH presented an interesting problem. The only possible source of the pyrene ring oxygen was from the C-5 position. This acted as an internal nucleophile, attacking the oxonium intermediate after its formation faster than the external nucleophile. This lead to the formation of a bicyclic system containing potential six and seven membered ring systems. As the six membered ring is the more stable of the two possibilities, attack by the hydride-equivalent at the anomeric position lead to ring opening forming a pyrenyl ring with an exocyclic diol. Subsequent generation of triflic acid during the reaction lead to the deprotection of the silyl ether (Scheme 6.17).

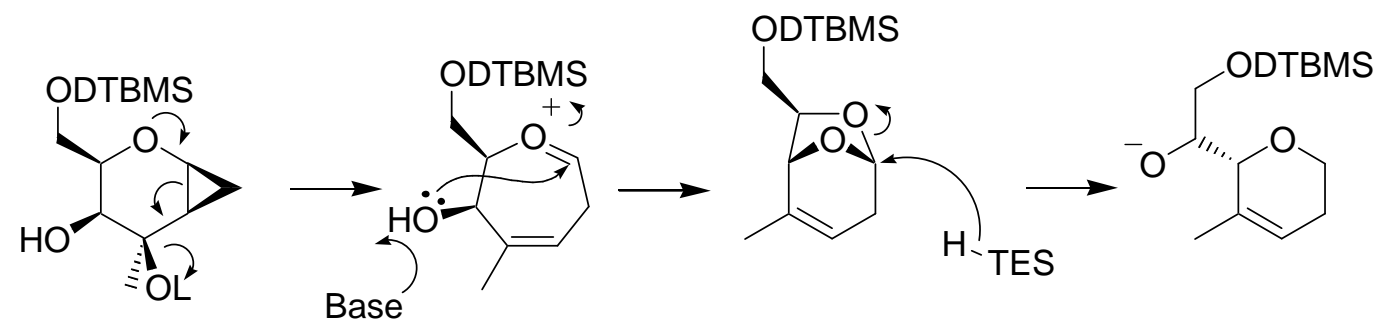

Scheme 6.17. Mechanism of formation of the cyclohexyl diol 6.7. 
The initial steps of this particular ring expansion/contraction were also observed in the formation of compound 2.7a. However in this case the presence of TESH resulted in further conversion of the $[3,2,1]$ bicyclic system to the observed diol 6.7 .

To determine if this was the correct mechanism, deuteride was then substituted for hydride in the ring expansion step. TESD was again synthesised according to the method of Doyle. ${ }^{97}$ Reaction with cyclopropane $\mathbf{6 . 4}$ in acetonitrile as before gave epimeric diols 6.17 and $\mathbf{6 . 1 8}$ as a 6:1 mixture of products with the major product being that shown in Figure 6.6. ${ }^{13} \mathrm{C}$ NMR revealed that the deuterium had only substituted at C-1. The C-1 carbon signal at $\delta_{\mathrm{C}} 63.47$ was a triplet with a coupling constant of 21.7 $\mathrm{Hz}$, indicative of an attached deuterium.<smiles>[2H]C1CC=C(C)[C@@H](C(O)CO)O1</smiles>

6.17

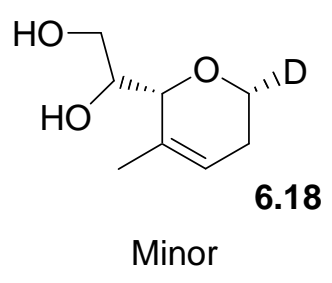

Figure 6.6. Major and minor products from reaction of 6.7 with TESD.

The stereochemistry of the reaction was confirmed by NOESY data that revealed an enhancement between the C-1 and C-5 protons on the minor isomer diol 6.19 (Figure 6.7) which, while it was not separated from the mixture, was clearly observable in the ${ }^{1} \mathrm{H}$ NMR. Diol 6.19 could also be detected in the ${ }^{13} \mathrm{C}$ NMR $\left(\delta_{\mathrm{C}} 63.51\right)$ as a series of 3 small peaks under the major epimer C-1 carbon with a coupling constant of $22.2 \mathrm{~Hz}$.

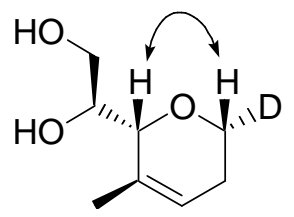

Figure 6.7. NOE enhancements for minor product diol 6.19. 
This agrees with the expected outcome of our proposed mechanism. The major product from the ring expansion is the more thermodynamically stable pyran ring and steric effects play little role in the reaction.

\subsection{Summary.}

An attempt was made to use the ring expansion methodology as part of the synthesis of a natural product. Modification of the cyclopropanated ring system was simple and high yielding. However the ring expansion reaction did not proceed as planned leading to an unexpected product which scuttled the overall synthesis. However a previously unknown series of pyrenes were synthesised and characterised. 


\section{Chapter 7: Conclusion}

At the commencement of this project a set of aims and objectives were set down. These objectives were met with varying degrees of success.

The synthesis of the various glycals involved in the project was optimised to provide good to excellent yields of most starting materials in multi-gram quantities, demonstrating that the chemistry is both efficient and scalable.

The glycals synthesised were then used to probe the application of the Ferrier rearrangement to the synthesis of seven-membered oxacycles with a view towards improving the stereoselectivity of the ring expansion compared to previous work. Different silyl protecting groups were investigated. It was found that the bis-tethered ditert-butylsilyl protecting group was superior to the mono tethered silyl protecting groups investigated due to the former's higher stability under the reaction conditions used for the ring expansions, as the mono-tethered groups TIPS and TBDPS were prone to deprotection. A variety of leaving groups were investigated, with the previously used acetate proving the most successful due to its reliability under the reaction conditions. The rest proved either too unstable to work with (triflate and mesylate) or too stable to be of further use (carbonate).

It was found that the stereoselectivity of the ring expansion was somewhat dependent on the glycal used as the substrate. Galactal in combination with the di-tert-butylsilyl gave the best results both in terms of yield and selectivity. Glucal in combination with TBDPS gave similar selectivity's with lower yields and was prone to unexpected rearrangements. A range of nucleophiles were successfully used in the ring expansions, 
with carbon nucleophiles giving the best selectivity. Of particular note was that $\mathrm{O}-$ nucleophiles failed to react.

Reaction products were characterised by NMR and Mass spectrometry to further investigate the mechanism of the rearrangement. This was consistant with that published for the Ferrier rearrangement i.e. the formation of an oxonium ion followed with interception by a suitable nucleophile to form the product oxepine. Side reactions were investigated and yielded more information about subsequent rearrangements of the oxepine ring. This was obtained with a combination of reaction condition manipulation and deuterium labelling experiments. One general mechanism with two reaction pathways was postulated to account for the observed products.

The controlled reactivity of the oxepine ring system was also examined to determine its suitability as a synthon in organic synthesis. A range of elaborations of the oxepine alkene were performed. The products were easily separated and prediction of the products stereo- and region-selectivity was relatively straightforward in most cases. The resulting oxepanes proved amenable to further elaboration, demonstrating their underlying stability to a range of reaction conditions.

Finally, an attempt was made to synthesise a naturally occurring compound. The deceptively simple target molecule proved ultimately elusive due to a previously unobserved rearrangement that effectively scuttled the project.

Further work with these systems could look at several areas. The oxepines and derivatives synthesised as part of this project in Chapters 3 and 5 should be examined for biological activity. Ring expansions of more complex cyclopropanes could be 
investigated, possibly leading to stereoselective ring expansion in the later stages of a total synthesis to further expand upon the methodology. This was considered at one point during the attempted synthesis of the quince oxepine $\mathbf{6 . 1}$ as described in Chapter 6 (Figure 7.1).
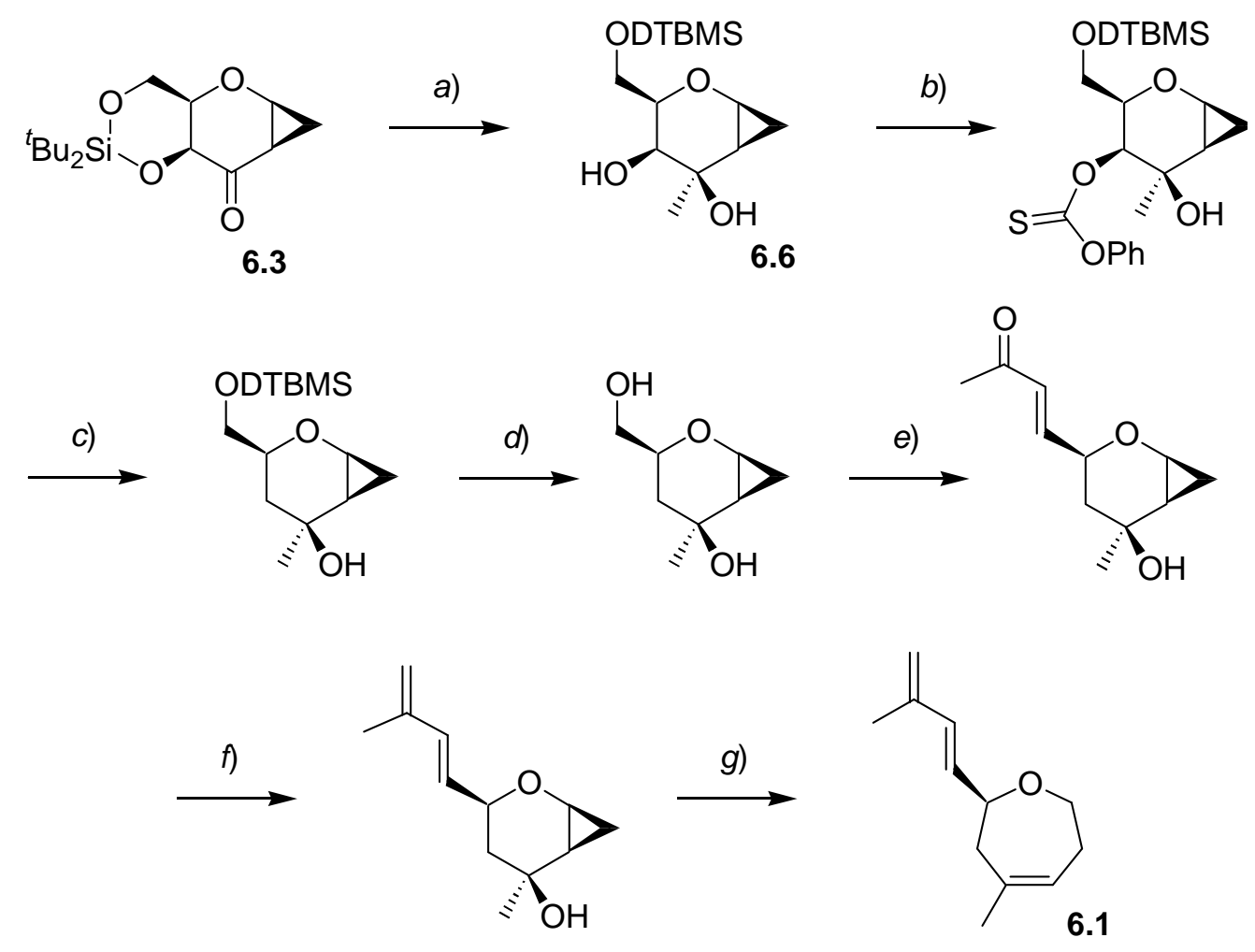

a) Grignard b) Xanthate formation. c) Barton-McCombie deoxygenation. d) Deprotection. e) Oxidation/Wittig. f) Wittig. $g$ ) Ring expansion.

Scheme 7.1. Possible alternative reaction scheme towards the formation of oxepine $\mathbf{6 . 1}$.

Starting with cyclopropane 6.6 the Barton-McCombie deoxygenation would be performed first to remove the $\mathrm{C}-4$ oxygen. This would hopefully remove the main cause of the failure of the total synthesis of $\mathbf{6 . 1}$ in Chapter 6. This would be followed by removal of the protecting group, followed by a one step oxidation/Wittig reaction to install the sidechain. A second Wittig reaction would complete the sidechain formation, leaving the ring expansion as the final step. However due to time constraints and the lack of vital starting materials to hand this alternate route was not attempted. 
Finally the cyclopropanated glycals should be further investigated as substrate molecules of interest in their own right. These investigations could include both radical and transition metal catalysed rearrangements. Some exploratory work has been done on radical rearrangements of simple cyclopropanes in similar systems by Gurjar and coworkers, ${ }^{125}$ which could be extended to the investigation of more complex systems. Palladium insertion into the cyclopropane has been extensively investigated by Madsen and Skaanderup in the case of platinum, ${ }^{48}$ but other transition metal catalysts could also be investigated. 


\section{Chapter 8: Experimental}

General: All melting points are uncorrected. Reaction progress was monitored using aluminium backed TLC plates pre-coated with silica UV 254 and visualised by UV radiation $(254 \mathrm{~nm})$ and either i) ceric ammonium molydate dip [ $\left(\mathrm{NH}_{4}\right)_{2} \mathrm{Mo}(\mathrm{VI}) .4 \mathrm{H}_{2} \mathrm{O}$, $12.5 \mathrm{~g} ; \mathrm{Ce}\left(\mathrm{SO}_{4}\right)_{2} .4 \mathrm{H}_{2} \mathrm{O}, 5 \mathrm{~g} ; 10 \%$ aq $\mathrm{H}_{2} \mathrm{SO}_{4}$ in $\left.\mathrm{H}_{2} \mathrm{O} 500 \mathrm{~mL}\right]$ or ii) $\left[5 \% \mathrm{H}_{2} \mathrm{SO}_{4}\right.$ in $\left.\mathrm{MeOH}\right]$ followed by heating. Flash chromatography was performed using silica gel 60 (220-240 mesh) with solvent systems as indicated. NMR spectra were recorded on Varian Inova 300, 500 and $600 \mathrm{MHz}$ systems, and were referenced to the relevant solvent peaks. Accurate masses were recorded on a Mariner time of flight spectrometer using positive and negative ionisation techniques. GC analyses of stereoselectivities were carried out on a Hewlett-Packard 6890 series instrument using a J\&W $30 \mathrm{~m}, 0.32 \mathrm{~mm}$ ID HP-1 column. Conditions for the determinations were an injection temperature of $100{ }^{\circ} \mathrm{C}$ held for 1 minute followed by a temperature ramp of $2{ }^{\circ} \mathrm{C}$ a minute to $200{ }^{\circ} \mathrm{C}$. Solvents and reagents from stock were purified by standard methods. Unless otherwise stated all reactions were performed under zero grade argon using previously dried reagents and solvents. THF and $\mathrm{Et}_{2} \mathrm{O}$ were freshly distilled from sodium under argon with benzophenone added as an indicator of dryness. Cold baths were generated by the use of dry ice slurries with appropriate solvents to generate the required temperatures.

The names of the compounds were generated with the assistance of the Beilstein on line database. 


\section{6-O-Triisopropylsilyl galactal (2.2).}

To galactal $2.1(806 \mathrm{mg}, 5.52 \mathrm{mmol})$ and $\mathrm{Et}_{3} \mathrm{~N}(1.677 \mathrm{~g}, 16.56 \mathrm{mmol})$ in DMF (15 mL) was added TIPSCl (1.166 $\mathrm{g}, 6.072 \mathrm{mmol})$ and the mixture stirred at ambient temperature for $3 \mathrm{~h}$. The mixture was poured onto EtOAc $(120 \mathrm{~mL})$ and washed with $\mathrm{H}_{2} \mathrm{O}(5 \times 50 \mathrm{~mL})$ and dried over $\mathrm{MgSO}_{4}$. Concentration in vacuo followed by flash chromatography on silica gel with hexanes:EtOAc (5:1) gave 2.2 as a clear oil $(1.104 \mathrm{~g}$, $66 \%$ yield).

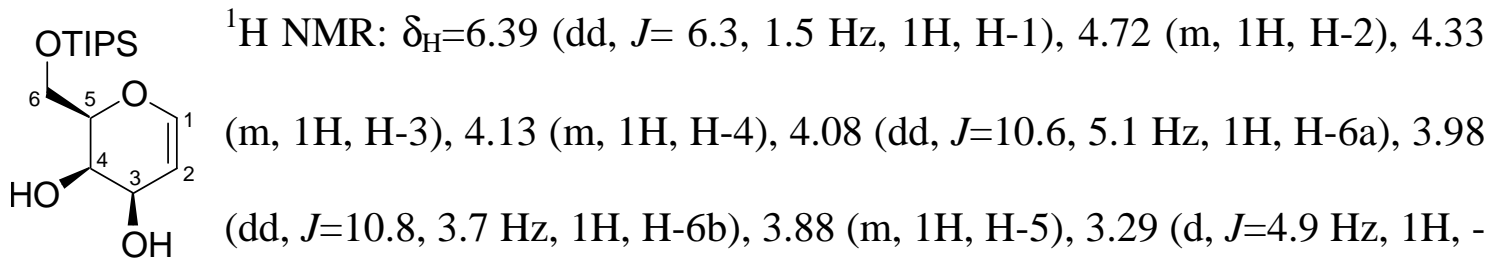
OH), $2.64(\mathrm{~d}, J=10.2 \mathrm{~Hz}, 1 \mathrm{H},-\mathrm{OH}), 1.08(\mathrm{~m}, 21 \mathrm{H}, \mathrm{H}-\mathrm{TIPS})$

\section{$(1 S, 3 R, 4 R, 5 R, 6 S)-3$-Triisopropylsilanyloxymethyl-2-oxa-bicyclo[4.1.0]heptane-4,5-diol} (2.3).

To silyl ether $2.2(437 \mathrm{mg}, 1.45 \mathrm{mmol})$ in $\mathrm{CH}_{2} \mathrm{Cl}_{2}$ at $0{ }^{\circ} \mathrm{C}$ was added dropwise $\mathrm{Et}_{2} \mathrm{Zn}$ (1M in hexanes, $7.8 \mathrm{~mL}, 7.8 \mathrm{mmol})$ and then $\mathrm{CH}_{2} \mathrm{I}_{2}(628 \mu \mathrm{L}, 7.8 \mathrm{mmol})$ dropwise. The mixture was stirred for 15 min and refluxed overnight. The reaction was quenched with saturated $\mathrm{NH}_{4} \mathrm{Cl}(30 \mathrm{~mL})$, extracted with $\mathrm{Et}_{2} \mathrm{O}(3 \mathrm{x} 30 \mathrm{~mL})$ and dried over $\mathrm{MgSO}_{4}$. Concentration in vacuo followed by flash chromatography on silica gel with hexanes: EtOAc (3:1) gave $\mathbf{2 . 3}$ as a white solid (331 $\mathrm{mg}, \mathbf{7 2 \%}$ ).

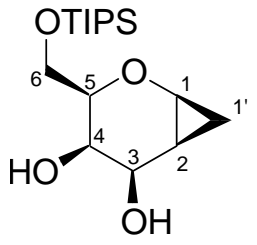

${ }^{1} \mathrm{H}$ NMR: $\delta_{\mathrm{H}}=4.12(\mathrm{dd}, J=6.6,5.3 \mathrm{~Hz}, 1 \mathrm{H}, \mathrm{H}-3), 4.04(\mathrm{~d}, J=5.1 \mathrm{~Hz}, 1 \mathrm{H}$, H-4), 3.94 (dd, $J=10.5,4.7 \mathrm{~Hz}, 1 \mathrm{H}, \mathrm{H}-6 \mathrm{a}), 3.87$ (dd, $J=10.7,3.6 \mathrm{~Hz}, 1 \mathrm{H}$, H-6b), 3.81 (m, 1H, H-1), 3.31 (t, J=4.2 Hz, 1H, H-5), 1.25 (dd, $J=10.3$, 7.1 Hz, 1H, H-2), 1.09 (m, 3H, TIPS- $\underline{\mathrm{CH}}-), 1.06$ (d, J=1.9 Hz, 9H, TIPS- $\left.\underline{\mathrm{CH}}_{3}\right), 1.05$ (d, $\left.J=2.2 \mathrm{~Hz}, 9 \mathrm{H}, \mathrm{TIPS}-\underline{\mathrm{CH}}_{3}\right), 0.65$ (dt, J=10.0, $\left.5.6 \mathrm{~Hz}, 2 \mathrm{H}, \mathrm{H}-1^{\prime}\right)$. 
${ }^{13} \mathrm{C}$ NMR: $\delta_{\mathrm{C}}=76.2(\mathrm{C}-5), 69.4(\mathrm{C}-4), 65.7$ (C-3), 64.3 (C-6), 54.5 (C-1), 17.9 (TIPS$\left.\underline{\mathrm{CH}}_{3}\right), 17.8\left(\mathrm{TIPS}-\underline{\mathrm{CH}}_{3}\right), 16.6$ (C-2), 11.8 (C-1'), 11.7 (TIPS- $\underline{\mathrm{CH}}-$ ).

MP: $83{ }^{\circ} \mathrm{C}$.

\section{$(1 S, 3 R, 4 R, 5 R, 6 S)-5$-acetoxy-4-hydroxy-3-triisopropylsilanyloxymethyl-2-oxa-}

\section{bicyclo[4.1.0]heptane (2.4).}

To cyclopropane 2.3 (100 mg, $0.284 \mathrm{mmol})$ in pyridine $(3 \mathrm{~mL})$ was added $\mathrm{Ac}_{2} \mathrm{O}(55 \mu \mathrm{l}$, $0.58 \mathrm{mmol})$ and DMAP (1.8 $\mathrm{mg}$, cat.). The reaction was stirred at ambient temperature for $2 \mathrm{~h}$. The mixture was poured onto $\mathrm{Et}_{2} \mathrm{O}(50 \mathrm{~mL})$ and washed with water $(2 \times 50$ $\mathrm{mL}$ ), dried over $\mathrm{MgSO}_{4}$ and concentrated in vacuo. Flash chromatography on silica gel with hexanes:EtOAc (3:1) gave $\mathbf{2 . 4}$ as a clear oil (110 $\mathrm{mg}$, 99\% yield).

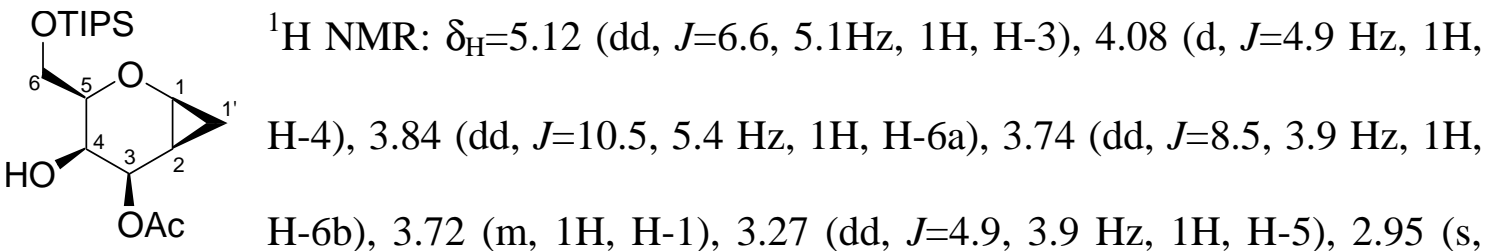
1H, -OH), 2.09 (s, 3H, H-Ac), 1.36 (m, 1H, H-2), 1.21 (m, 1H, H-1'), 0.96 (m, 21H, TIPS- $\underline{\mathrm{CH}}-$, TIPS- $\left.\underline{\mathrm{CH}}_{3}\right), 0.60$ (dt, J=9.8, $\left.5.6 \mathrm{~Hz}, 1 \mathrm{H}, \mathrm{H}-1^{\prime}\right)$

\section{$(1 \mathrm{a} S, 3 R, 3 \mathrm{a} R, 6 \mathrm{a} R, 6 \mathrm{~b} R)-3$-triisopropylsilanyloxymethyl-hexahydro-2,4,6-trioxa- cyclopropa[e]inden-5-one (2.5).}

To 2.3 (100 mg, 0.284mmol) in THF (2 mL) was added carbonyldiimidazole (52 mg, $0.32 \mathrm{mmol})$ and imidazole $(1$ crystal). The reaction mixture was stirred for $30 \mathrm{~min}$ at ambient temperature and then a further portion of carbonyldiimidazole $(26 \mathrm{mg}, 0.16$ $\mathrm{mmol})$ added and the reaction stirred for $36 \mathrm{~h}$. The reaction was poured onto $\mathrm{Et}_{2} \mathrm{O}(50$ $\mathrm{mL})$ and washed with water $(3 \mathrm{x} 50 \mathrm{~mL})$, dried over $\mathrm{MgSO}_{4}$ and concentrated in vacuo. Flash chromatography in hexane:EtOAc (1:1) gave 2.5 as a clear oil (58 mg, 54\%) and recovered starting material (44 $\mathrm{mg}, 44 \%)$. 


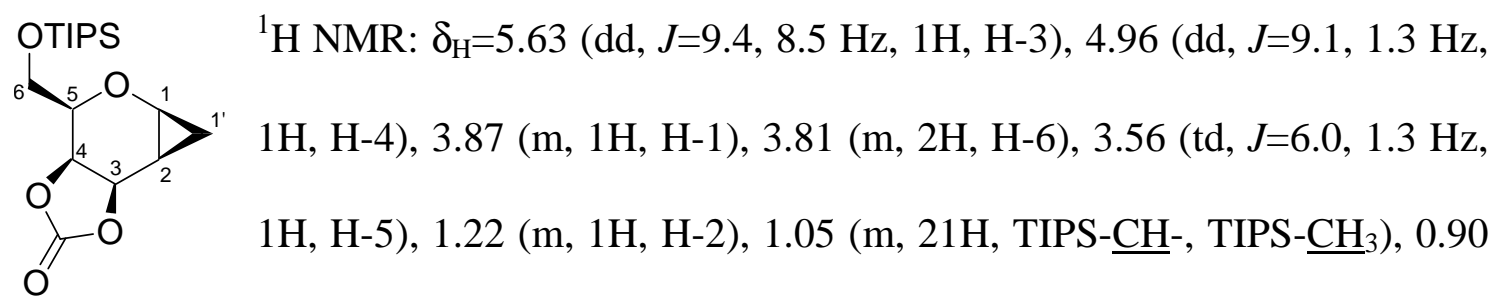
(m, 2H, H-1').

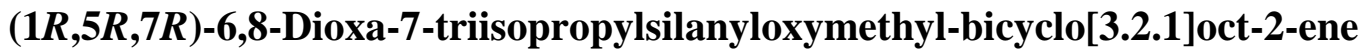
(2.7).

To cyclopropane $2.3(150 \mathrm{mg}, 0.43 \mathrm{mmol})$ in a $1: 1 \mathrm{mix}$ of $\mathrm{CH}_{2} \mathrm{Cl}_{2}$ and pyridine $(2 \mathrm{~mL})$ at $0{ }^{\circ} \mathrm{C}$ was added $\mathrm{Tf}_{2} \mathrm{O}(181 \mu \mathrm{L}, 1.075 \mathrm{mmol})$ dropwise, and the reaction kept at $0{ }^{\circ} \mathrm{C}$ over $24 \mathrm{~h}$. The mixture was poured onto $\mathrm{Et}_{2} \mathrm{O}(10 \mathrm{~mL})$ and washed with water $(4 \mathrm{x}$ $10 \mathrm{~mL})$, dried $\left(\mathrm{MgSO}_{4}\right)$ and concentration in vacuo. Flash chromatography on silica gel with hexanes:EtOAc (3:1) gave 2.7 as a clear oil (17 mg, 12\%).

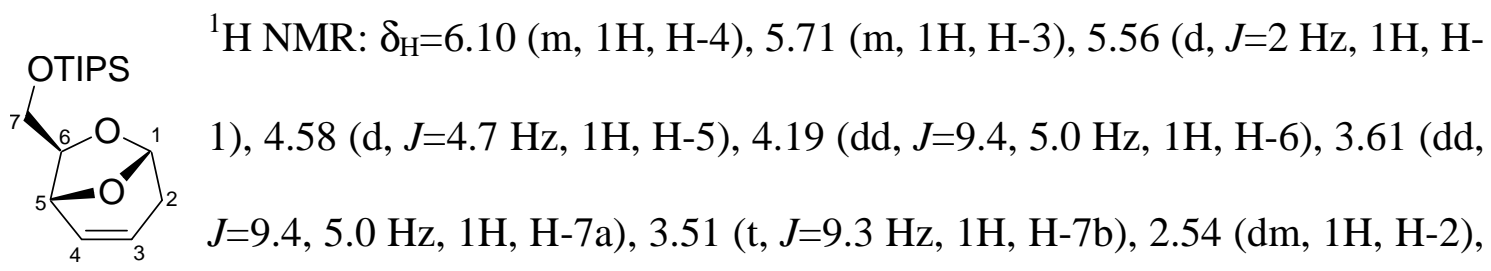

2.08 (dm, 1H, H-2b), 1.09 (m, 21H, TIPS- $\underline{\mathrm{CH}}-$, TIPS- $\left.\underline{\mathrm{CH}_{3}}\right)$.

${ }^{13} \mathrm{C}$ NMR: $\delta_{\mathrm{C}}=129.1(\mathrm{C}-4), 124.2(\mathrm{C}-3), 100.7$ (C-1), 84.8 (C-6), 71.6 (C-5), 63.5 (C-7), 34.2 (C-2), 17.9 (TIPS- $\underline{\mathrm{CH}} 3$ ), 17.7 (TIPS- $\underline{\mathrm{CH}_{3}}$ ), 12.2 (TIPS- $\underline{\mathrm{CH}}-$ ), 11.9 (TIPS- $\underline{\mathrm{CH}}-$ ).

ES HRMS $m / z$ : calculated for $\mathrm{C}_{16} \mathrm{H}_{30} \mathrm{O}_{3} \mathrm{Si}+\mathrm{H}$ 299.2042. Found 299.1821.

$(1 S, 3 R, 4 R, 5 S, 6 S)$-5-Azido-3-triisopropylsilanyloxymethyl-2-oxa-bicyclo[4.1.0]heptan-4-ol (2.9).

To a mixture of cyclopropane $2.3(80 \mathrm{mg}, 0.25 \mathrm{mmol}), \mathrm{PPh}_{3}(73 \mathrm{mg}, 0.28 \mathrm{mmol})$ and $\mathrm{TMSN}_{3}(145 \mathrm{mg}, 1.265 \mathrm{mmol})$ in THF $(2 \mathrm{~mL})$ at $0{ }^{\circ} \mathrm{C}$ was added DEAD $(48 \mathrm{mg}$, $0.28 \mathrm{mmol}$ ). The reaction was warmed to ambient temperature and stirred for $12 \mathrm{~h}$. The mixture was poured onto $\mathrm{Et}_{2} \mathrm{O}(10 \mathrm{~mL})$, washed with saturated aq $\mathrm{NaHCO}_{3}(10 \mathrm{~mL})$ 
and water $(3 \times 10 \mathrm{~mL})$ and dried over $\mathrm{MgSO}_{4}$. Concentration in vacuo gave 2.9 as white solid $(85 \%)$.

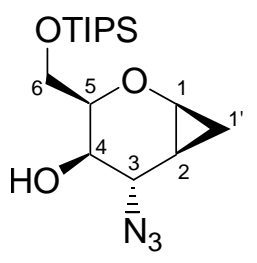

${ }^{1} \mathrm{H}$ NMR: $\delta_{\mathrm{H}}=4.11(\mathrm{t}, J=5.4 \mathrm{~Hz}, 1 \mathrm{H}, \mathrm{H}-3), 4.03(\mathrm{~d}, J=5.1 \mathrm{~Hz}, 1 \mathrm{H}, \mathrm{H}-4)$, $3.92(\mathrm{dd}, J=10.5,5.2 \mathrm{~Hz}, 1 \mathrm{H}, \mathrm{H}-6 \mathrm{a}), 3.85$ (dd, J=10.5, 4.1 Hz, 1H, H6b), 3.79 (ddd, J=6.8, 5.6, 2.7 Hz, 1H, H-1), 3.30 (t, $J=4.4 \mathrm{~Hz}, 1 \mathrm{H}, \mathrm{H}-5$ ), $1.22(\mathrm{~m}, 1 \mathrm{H}, \mathrm{H}-2), 1.05$ (m, 21H, TIPS- $\underline{\mathrm{CH}}-$, TIPS- $\left.\underline{\mathrm{CH}}_{3}\right), 0.63(\mathrm{dt}, J=10.0,5.6 \mathrm{~Hz}, 2 \mathrm{H}$, H-1').

${ }^{13} \mathrm{C}$ NMR: $\delta_{\mathrm{C}}=76.3(\mathrm{C}-5), 69.2(\mathrm{C}-4), 65.6(\mathrm{C}-4), 64.4(\mathrm{C}-6), 54.4(\mathrm{C}-1), 17.9$ (TIPS$\left.\underline{\mathrm{CH}}_{3}\right), 17.8$ (TIPS- $\left.\underline{\mathrm{CH}}_{3}\right), 16.6$ (C-2), 11.8 (TIPS- $\underline{\mathrm{CH}}-$ ), 11.7 (C1').

MP: $85{ }^{\circ} \mathrm{C}$.

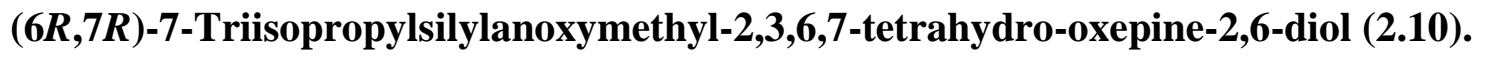

To a mixture of cyclopropane 2.3 (78 mg, $0.24 \mathrm{mmol}), \mathrm{PPh}_{3}(94 \mathrm{mg}, 0.0 .36 \mathrm{mmol})$ and TMSallyl $(76 \mu \mathrm{L}, 0.48 \mathrm{mmol})$ in $\mathrm{CH}_{2} \mathrm{Cl}_{2}(2 \mathrm{~mL})$ at $0{ }^{\circ} \mathrm{C}$ was added DEAD (30 $\mathrm{mg}$, $0.176 \mathrm{mmol})$ dropwise and stirred for $2 \mathrm{~h}$. The mixture was poured onto $\mathrm{CH}_{2} \mathrm{Cl}_{2}(10$ $\mathrm{mL})$, washed with saturated aq $\mathrm{NaHCO}_{3}(10 \mathrm{~mL})$ and water $(4 \times 10 \mathrm{~mL})$, dried over $\mathrm{MgSO}_{4}$ and concentrated in vacuo. Flash chromatography on silica gel with hexanes:EtOAc (3:1) gave $\mathbf{2 . 1 0}$ as a clear oil $(13 \mathrm{mg}, 17 \%)$ as a 1:1 mixture of epimers by GC.<smiles>OC1CC=CC(O)C(COC(F)(F)F)O1</smiles>

${ }^{1} \mathrm{H}$ NMR: $\delta_{\mathrm{H}}=5.85(\mathrm{~m}, 1 \mathrm{H}, \mathrm{H}-3), 5.80(\mathrm{~m})$ and $5.72(\mathrm{~m})(1 \mathrm{H}, \mathrm{H}-4)$, $5.41(\mathrm{~s})$ and $5.10(\mathrm{dd}, J=6.2,2.7 \mathrm{~Hz})(1 \mathrm{H}, \mathrm{H}-1), 4.47(\mathrm{bs}, 1 \mathrm{H}, \mathrm{H}-5)$, 3.75 (m, 2H, H-7), 3.68 (m, 2H, H-6), 3.38 (s) and $3.00(\mathrm{~s})(1 \mathrm{H},-\mathrm{OH})$, $2.68(\mathrm{~s}, 1 \mathrm{H}-\mathrm{OH}), 2.43(\mathrm{~m})$ and $2.34(\mathrm{~m})(1 \mathrm{H}, \mathrm{H}-2 \mathrm{a}), 2.18(\mathrm{~m})$ and $2.12(\mathrm{~m})(1 \mathrm{H}, \mathrm{H}-2 \mathrm{~b})$, $1.07\left(\mathrm{~m}, 21 \mathrm{H}\right.$, TIPS- $\underline{\mathrm{CH}}-$, TIPS- $\left.\underline{\mathrm{CH}_{3}}\right)$. 

(2.11).

a) To a mixture of cyclopropane 2.3 (50 $\mathrm{mg}, 0.16 \mathrm{mmol}), \mathrm{PPh}_{3}(46 \mathrm{mg}, 0.176 \mathrm{mmol})$ and $\mathrm{TMSN}_{3}(91 \mathrm{mg}, 0.8 \mathrm{mmol})$ in DMF $(2 \mathrm{~mL})$ at $0{ }^{\circ} \mathrm{C}$ was added DEAD $(30 \mathrm{mg}, 0.176$ mmol) dropwise and stirred for $2 \mathrm{~h}$. The mixture was poured onto $\mathrm{Et}_{2} \mathrm{O}(10 \mathrm{~mL})$, washed with saturate aq $\mathrm{NaHCO}_{3}(10 \mathrm{~mL})$ and water $(4 \times 10 \mathrm{~mL})$, dried over $\mathrm{MgSO}_{4}$ and concentrated in vacuo. Flash chromatography on silica gel with hexanes:EtOAc (5:1) gave 2.11 as a clear oil (15 mg, $27 \%)$ in a 4:1 mix of $\alpha$ and $\beta$ epimers.

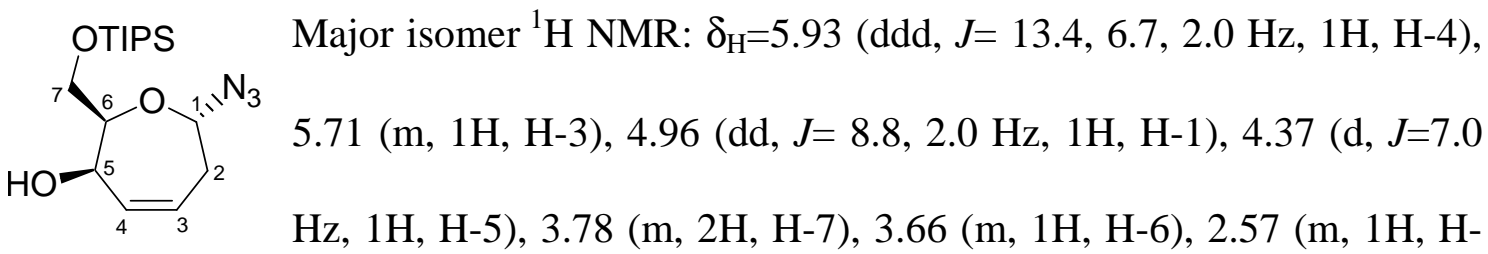
2a), 2.34 (ddd, $J=16.5,7.2,2.1 \mathrm{~Hz}, 1 \mathrm{H}, \mathrm{H}-2 \mathrm{~b}), 1.07$ (m, 21H, TIPS- $\underline{\mathrm{CH}}-$, TIPS- $\underline{\mathrm{CH}}_{3}$ ). ${ }^{13} \mathrm{C}$ NMR: $\delta_{\mathrm{C}}=133.5(\mathrm{C}-4), 128(\mathrm{C}-3), 91(\mathrm{C}-1), 82(\mathrm{C}-6), 68(\mathrm{C}-5), 62.5(\mathrm{C}-7), 36(\mathrm{C}-$ 2), 18.0 (TIPS- $\underline{\mathrm{CH}} 3$ ), 12.0 (TIPS- $\underline{\mathrm{CH}}-$ ).

b) To a mixture of 2.3 (125 mg, $0.4 \mathrm{mmol}), \mathrm{PPh}_{3}(157 \mathrm{mg}, 0.6 \mathrm{mmol})$ and $\mathrm{TMSN}_{3}(228$ $\mathrm{mg}, 2 \mathrm{mmol})$ in DMF (2 mL) at $-20{ }^{\circ} \mathrm{C}$ was added DEAD (104 mg, $\left.0.6 \mathrm{mmol}\right)$ dropwise and stirred for $2 \mathrm{~h}$. The mixture was poured onto $\mathrm{Et}_{2} \mathrm{O}(10 \mathrm{~mL})$, washed with saturated aq $\mathrm{NaHCO}_{3}(10 \mathrm{~mL})$ and water $(4 \times 10 \mathrm{~mL})$, dried dried over $\mathrm{MgSO}_{4}$ and concentrated in vacuo. Flash chromatography on silica gel with hexanes:EtOAc (5:1) gave $\mathbf{2 . 1 2}$ as a clear oil (60 mg, $44 \%$ ) as a 20:1 mix of $\alpha$ and $\beta$ epimers. NMR data as above.

\section{Reaction of cyclopropane 1.61 with the leaving group generated in situ.}

To a solution of cyclopropane $1.61(100 \mathrm{mg}, 0.29 \mathrm{mmol})$ in $\mathrm{MeCN}(2 \mathrm{~mL})$ at $-40{ }^{\circ} \mathrm{C}$ was added $\mathrm{TMSN}_{3}(230 \mu \mathrm{L}, 1.45 \mathrm{mmol})$ and TMSOTf $(60 \mu \mathrm{L}, 0.35 \mathrm{mmol})$. The reaction mixture was stirred and allowed to warm to $0{ }^{\circ} \mathrm{C}$ until complete by TLC $(2 \mathrm{~h})$. 
The mixture was quenched with saturated aq $\mathrm{NaHCO}_{3}(10 \mathrm{~mL})$, extracted with $\mathrm{Et}_{2} \mathrm{O}(2$ x $20 \mathrm{~mL}$ ), dried over $\mathrm{MgSO}_{4}$ and concentrated in vacuo. Flash chromatography on silica gel with hexanes:EtOAc (20:1) gave three compounds.

[(4aR,9aS)-2,2-tert-Butyl-4,4a,7,9a-tetrahydro-1,3,5-trioxa-2-sila-benzocyclohepten -(6E)-ylidene]-trimethylsilanyl-amine (2.13).

${ }^{1} \mathrm{H}$ NMR: $\delta_{\mathrm{H}}=5.67(\mathrm{~m}, 1 \mathrm{H}, \mathrm{H}-4), 5.60(\mathrm{~m}, 1 \mathrm{H}, \mathrm{H}-3), 4.50(\mathrm{t}$,
$J=8.3 \mathrm{~Hz}, 1 \mathrm{H}, \mathrm{H}-5), 3.99(\mathrm{dd}, J=10.8,4.9 \mathrm{~Hz}, 1 \mathrm{H}, \mathrm{H}-7), 3.83$ (t, $J=10.3 \mathrm{~Hz}, 1 \mathrm{H}, \mathrm{H}-7), 3.53$ (td, $J=10.0,4.9 \mathrm{~Hz}, 1 \mathrm{H}, \mathrm{H}-6), 3.33$ (dd, $J=18.3,6.6 \mathrm{~Hz}$, 1H, H-2), 3.26 (dd, J=17.7, $6.4 \mathrm{~Hz}, 1 \mathrm{H}, \mathrm{H}-2), 1.07$ (s, 9H, H- $\left.{ }^{t}{ }^{\mathrm{Bu}}-\underline{\mathrm{CH}}_{3}\right), 1.01$ (s, 9H, H$\left.{ }^{t} \mathrm{Bu}-\underline{\mathrm{CH}}_{3}\right), 0.12\left(\mathrm{~s}, 9 \mathrm{H}, \mathrm{TMS}-\underline{\mathrm{CH}}_{3}\right)$.

${ }^{13} \mathrm{C}$ NMR: $\delta_{\mathrm{C}}=135.5(\mathrm{C}-4), 120.2(\mathrm{C}-3), 118.1$ (C-1), 75.1 (C-5), 70.5 (C-6), 68.5 (C-7), $27.4\left(\mathrm{C}-{ }^{t} \mathrm{Bu}-\underline{\mathrm{CH}}_{3}\right), 27.0\left(\mathrm{C}-{ }^{t} \mathrm{Bu}-\underline{\mathrm{CH}}_{3}\right), 22.6$ (Si-C), 19.9 (Si-C), 16.7 (C-2), 0.1 (C-TMS). ES HRMS m/z: calculated for $\mathrm{C}_{18} \mathrm{H}_{35} \mathrm{NO}_{3} \mathrm{Si}_{2}+\mathrm{NH}_{4}$ 387.2494. Found $\left(\mathrm{M}+\mathrm{NH}_{4}\right)^{+}$ 387.2484 .

IR: $2934,2860,2108,1472,1253,1092,839,824,733 \mathrm{~cm}^{-1}$.

\section{(4S,5R)-2,2-Di-tert-butyl-4-((Z)-4,4-diazidobut-1-enyl)-[1,3,2]dioxasilinan-5-ol} (2.14).

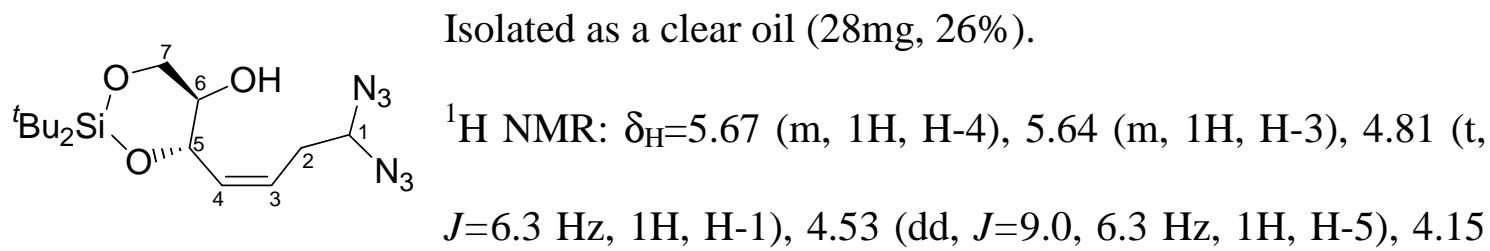
(dd, $J=10.5,4.7 \mathrm{~Hz}, 1 \mathrm{H}, \mathrm{H}-7), 3.86$ (t, $J=10.5 \mathrm{~Hz}, 1 \mathrm{H}, \mathrm{H}-7), 3.58$ (td, $J=9.1,4.7 \mathrm{~Hz}$, 1H, H-6), 2.59 (m, 2H, H-2), 2.05 (bs, 1H, -OH), 1.06 (s, 9H, H- $\left.{ }^{t} \mathrm{Bu}-\underline{\mathrm{CH}}_{3}\right), 0.99$ (s, 9H, $\left.\mathrm{H}-{ }^{t} \mathrm{Bu}-\underline{\mathrm{CH}}_{3}\right)$. 
${ }^{13} \mathrm{C}$ NMR: $\delta_{\mathrm{C}}=134.8(\mathrm{C}-4), 126.2(\mathrm{C}-3), 77.3(\mathrm{C}-1), 74.5(\mathrm{C}-5), 69.7$ (C-6), $67.8(\mathrm{C}-7)$, 33.2 (C-2), $27.4\left(\mathrm{C}-{ }^{t} \mathrm{Bu}-\underline{\mathrm{CH}}_{3}\right), 27.1\left(\mathrm{C}-{ }^{t} \mathrm{Bu}-\underline{\mathrm{CH}}_{3}\right), 22.6$ (Si-C), 20.0 (Si-C).

IR: 3431, 2934, 2860, 2099, 1473, 1213, 1136, 1062, 908, 824, $731 \mathrm{~cm}^{-1}$.

\section{(Z)-4-((4S,5R)-2,2-Di-tert-butyl-5-hydroxy[1,3,2]dioxasilinan-4-yl)-but-3-enenitrile}

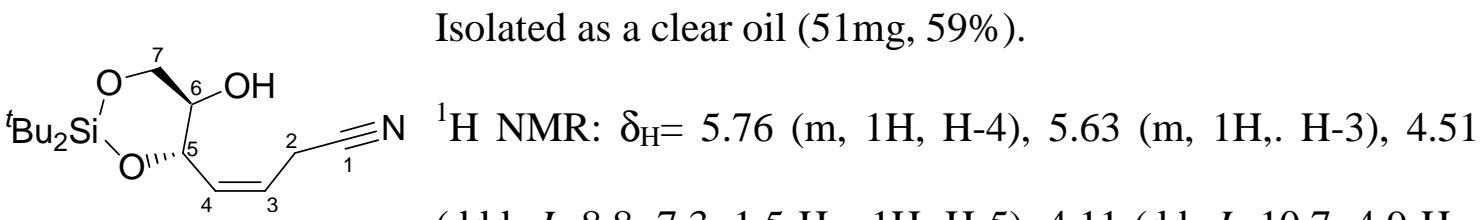
(ddd, $J=8.8,7.3,1.5 \mathrm{~Hz}, 1 \mathrm{H}, \mathrm{H}-5), 4.11$ (dd, $J=10.7,4.9 \mathrm{~Hz}$,

1H, H-7), 3.85 (t, J=10.5 Hz, 1H, H-7), 3.59 (td, J=10.0, $4.7 \mathrm{~Hz}, 1 \mathrm{H}, \mathrm{H}-6), 3.30$ (d, $J=7.1,1.7 \mathrm{~Hz}, 2 \mathrm{H}, \mathrm{H}-2), 2.35$ (bs, 1H, $-\mathrm{OH}), 1.06$ (s, 9H, H- $\left.{ }^{t}{ }^{\mathrm{Bu}}-\underline{\mathrm{CH}}_{3}\right), 0.99$ (s, 9H, H$\left.{ }^{t} \mathrm{Bu}-\underline{\mathrm{CH}}_{3}\right)$.

${ }^{13} \mathrm{C}$ NMR: $\delta=135.4$ (C-4), 120.8 (C-3), 118.1 (C-1), 74.7 (C-5), 69.6 (C-6), 68.0 (C-7), $27.4\left(\mathrm{C}-{ }^{t} \mathrm{Bu}-\underline{\mathrm{CH}}_{3}\right), 27.0\left(\mathrm{C}-{ }^{t} \mathrm{Bu}-\underline{\mathrm{CH}}_{3}\right), 22.6$ (Si-C), 20.0 (Si-C), 16.8 (C-2).

IR: $3435,2934,2860,2254,2113,1473,1134,1064,907,824,729 \mathrm{~cm}^{-1}$.

\section{1-((4aR,9aS)-2,2-tert-Butyl-4a,6,7,9a-tetrahydro-(4H)-1,3,5-trioxa-2-sila-benzo}

cyclohepten-6-yl)-propan-2-one (2.16)

To a solution of $\mathbf{1 . 6 1}(222 \mathrm{mg}, 0.65 \mathrm{mmol})$ in a mixture of THF (6 mL) and pyridine $(1.5 \mathrm{~mL})$ at $-40{ }^{\circ} \mathrm{C}$ was added dropwise a solution of Tebbe's reagent in toluene (1.94 mmol, $0.5 \mathrm{M})$. After $2 \mathrm{~h}$ a further portion of Tebbe's reagent was added (1.3 mmol, 0.5 M) and the mixture stirred for $1 \mathrm{~h}$. The reaction was quenched at $-40{ }^{\circ} \mathrm{C}$ with $0.1 \mathrm{M}$ $\mathrm{NaOH}$ until evolution of gas was complete. It was then diluted with hexane $(30 \mathrm{~mL})$ and filtered through a silica plug. Flash chromatography on silica gel with hexanes:EtOAc 9:1 containing 2\% TEA gave a pale yellow oil that was immediately transferred to a RBF fitted with a condenser, purged with argon and then refluxed with 
PhCN (2 mL). Flash chromatography on silica gel with hexanes:EtOAc (3:1) gave the oxepine $2.16(73 \mathrm{mg}, 33 \%)$ as a 1:1 mixture of epimers by NMR.

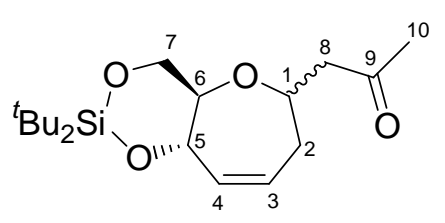

${ }^{1} \mathrm{H}$ NMR: $\delta_{\mathrm{H}}=5.80(\mathrm{~m})$ and $5.72(\mathrm{~m})(1 \mathrm{H}, \mathrm{H}-4), 5.65(\mathrm{~m}, 1 \mathrm{H}$, $\mathrm{H}-3), 4.76(\mathrm{~m})$ and $4.51(\mathrm{~m})(1 \mathrm{H}, \mathrm{H}-5), 4.42(\mathrm{~m}), 1 \mathrm{H}$ and $3.95(\mathrm{~m})(1 \mathrm{H}, \mathrm{H}-1), 3.90(\mathrm{~m})$ and $3.40(\mathrm{~m})(1 \mathrm{H}, \mathrm{H}-6), 3.78$ (m, 2H, H-7), $2.70(\mathrm{~m})$ and $2.40(\mathrm{~m})(2 \mathrm{H}, \mathrm{H}-8), 2.30(\mathrm{~m})$ and $2.15(\mathrm{~m})(2 \mathrm{H}, \mathrm{H}-2), 2.17$ (s, 3H, H-10), 2.15 (m, 3H, H-10), 1.03 (s) and 0.98 (s) (18H, $\left.\mathrm{H}^{t}{ }^{t} \mathrm{Bu}-\underline{\mathrm{CH}}_{3}\right)$.

${ }^{13} \mathrm{C}$ NMR: $\delta_{\mathrm{C}}=206.6(\mathrm{C}-9), 138.9$ and $136.1(\mathrm{C}-4), 124.75$ and $124.70(\mathrm{C}-3), 78.4$ and 72.7 (C-6), 77.7 and $75.6(\mathrm{C}-5), 77.2$ and $71.8(\mathrm{C}-1), 67.0$ and $66.7(\mathrm{C}-7), 50.2$ and 49.0 (C-8), 36.3 and 34.0 (C-2), 31.0 and 30.9 (C-10), 27.4 and $27.0\left(\mathrm{C}-{ }^{t} \mathrm{Bu}-\underline{\mathrm{CH}}_{3}\right), 19.9$ and $19.8\left(\mathrm{C}^{t}{ }^{t} \mathrm{Bu}-\underline{\mathrm{CH}}_{3}\right)$.

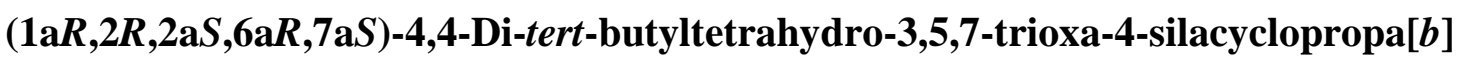

\section{naphthalene-2-ol (3.3).}

To galactal $3.1(890 \mathrm{mg}, 3.1 \mathrm{mmol})$ in $\mathrm{Et}_{2} \mathrm{O}(20 \mathrm{~mL})$ at $0{ }^{\circ} \mathrm{C}$ was added $\mathrm{Et}_{2} \mathrm{Zn}(1 \mathrm{M}$ in hexanes, $9.3 \mathrm{~mL}, 9.33 \mathrm{mmol})$ followed by $\mathrm{CH}_{2} \mathrm{I}_{2}(750 \mu \mathrm{L}, 9.33 \mathrm{mmol})$. The solution was stirred at $0{ }^{\circ} \mathrm{C}$ for $5 \mathrm{~h}$ and then one further aliquot of $\mathrm{Et}_{2} \mathrm{Zn}(1 \mathrm{M}$ in hexanes, 9.33mL, $9.33 \mathrm{mmol})$ and $\mathrm{CH}_{2} \mathrm{I}_{2}(750 \mu \mathrm{L}, 9.33 \mathrm{mmol})$ were added. After a further $4 \mathrm{~h}$ at $0{ }^{\circ} \mathrm{C}$, the solution was poured onto $\mathrm{NH}_{4} \mathrm{Cl}(100 \mathrm{~mL})$ and extracted with $\mathrm{Et}_{2} \mathrm{O}(2 \times 75$ $\mathrm{mL})$. The combined ether layers were washed with $\mathrm{H}_{2} \mathrm{O}(50 \mathrm{~mL})$, saturated brine $(50$ $\mathrm{mL}$ ) and dried with $\mathrm{MgSO}_{4}$. Concentration in vacuo followed by flash chromatography on silica gel with hexanes:EtOAc $(5: 1)$ gave of $\mathbf{3 . 3}$ as a white solid $(650 \mathrm{mg}, 70 \%)$ with traces of starting material.<smiles>CC(C)(C)[SiH2]O[C@H]1O[C@@H]2CO[C@@H]3C[C@H]3[C@H]2O1</smiles>

${ }^{1} \mathrm{H}$ NMR: $\delta_{\mathrm{H}}=4.38(\mathrm{~d}, J=5.9 \mathrm{~Hz}, 1 \mathrm{H}, \mathrm{H}-4), 4.20(\mathrm{~m}, 3 \mathrm{H}, \mathrm{H}-3, \mathrm{H}-$ 6), 3.82 (ddd, $J=4.1,2.9,1.5 \mathrm{~Hz}, 1 \mathrm{H}, \mathrm{H}-1$ ), 3.35, (bs, $1 \mathrm{H} \mathrm{H}-5$ ), $2.95(\mathrm{~d}, J=10.5 \mathrm{~Hz}, 1 \mathrm{H},-\mathrm{OH}), 1.25\left(\mathrm{~m}, 1 \mathrm{H}, \mathrm{H}-1^{\prime}\right), 1.20(\mathrm{~m}, 1 \mathrm{H}$, 
$\mathrm{H}-2), 1.03\left(\mathrm{~s}, 9 \mathrm{H}, \mathrm{H}^{t}{ }^{t} \mathrm{Bu}-\underline{\mathrm{CH}}_{3}\right), 1.01\left(\mathrm{~s}, 9 \mathrm{H}, \mathrm{H}-{ }^{t} \mathrm{Bu}-\underline{\mathrm{CH}}_{3}\right), 0.60$ (dt, J=9.7, 5.6 Hz, 1H, H1').

${ }^{13} \mathrm{C} \quad \mathrm{NMR} \quad \delta_{\mathrm{C}}=74.0(\mathrm{C}-5), 71.2(\mathrm{C}-4), 68.1(\mathrm{C}-6), 64.5(\mathrm{C}-3), 54.1(\mathrm{C}-1), 27.7$ (C- $\left.{ }^{t} \mathrm{Bu}-\underline{\mathrm{CH}}_{3}\right), 27.4\left(\mathrm{C}-{ }^{t} \mathrm{Bu}-\underline{\mathrm{CH}}_{3}\right), 23.3(\mathrm{Si}-\mathrm{C}), 15.6(\mathrm{C}-2), 10.8\left(\mathrm{C}-1^{\prime}\right)$.

ES HRMS m/z: calculated for $\mathrm{C}_{15} \mathrm{H}_{28} \mathrm{O}_{4} \mathrm{Si}+\mathrm{Na}$ : 323.1649. Found 323.1657.

IR: $3550,2984,2859,1473,1182,1148,1088,908,825,788 \mathrm{~cm}^{-1}$. MP: $74-75^{\circ} \mathrm{C}$.

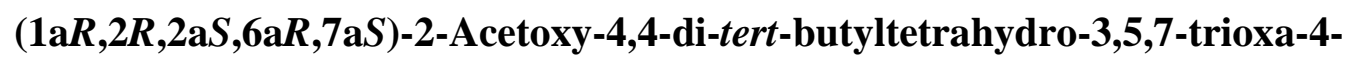
silacyclopropa $[b]$ naphthalene (3.4).

Compound 3.3 from above was dissolved in $\mathrm{CH}_{2} \mathrm{Cl}_{2}(20 \mathrm{~mL})$ then $\mathrm{Ac}_{2} \mathrm{O}(410 \mu \mathrm{L}, 4.33$ mmol), py $(525 \mu \mathrm{L}, 6.5 \mathrm{mmol})$ and a catalytic amount of DMAP were added. The solution was stirred for $2 \mathrm{~h}$ at ambient temperature, diluted with $\mathrm{CH}_{2} \mathrm{Cl}_{2}(20 \mathrm{~mL})$, washed with $\mathrm{H}_{2} \mathrm{O}(3 \times 50 \mathrm{~mL})$ then dried over $\mathrm{MgSO}_{4}$. Concentration in vacuo followed by flash chromatography on silica gel with hexanes:EtOAc (5:1) gave $\mathbf{3 . 4}$ as a white amorphous solid (692 mg, 93\%).

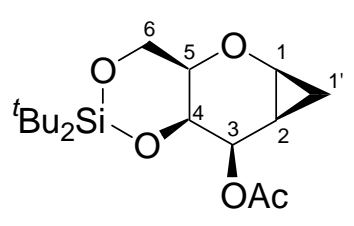

${ }^{1} \mathrm{H}$ NMR: $\delta_{\mathrm{H}}=5.17(\mathrm{dd}, J=7.0,5.1 \mathrm{~Hz}, 1 \mathrm{H}, \mathrm{H}-3), 4.70(\mathrm{~d}, J=5.4$ $\mathrm{Hz}, 1 \mathrm{H}, \mathrm{H}-4), 4.21(\mathrm{dd}, J=12.3,1.7 \mathrm{~Hz}, 1 \mathrm{H}, \mathrm{H}-6 \mathrm{a}), 4.14$ (dd, $J=12.4,2.6 \mathrm{~Hz}, 1 \mathrm{H}, \mathrm{H}-6 \mathrm{~b}), 3.82$ (m, 1H, H-1), 3.38 (m, 1H, H-5), 2.15 (s, 3H, H-Ac), 1.47 (m, 1H, H-1'), 1.18 (m, 1H, H-2), 1.05 (s, 9H, H- ${ }^{t} \mathrm{Bu}^{-\mathrm{CH}_{3}}$ ), $1.00\left(\mathrm{~s}, 9 \mathrm{H}, \mathrm{H}-{ }^{t} \mathrm{Bu}-\underline{\mathrm{CH}}_{3}\right), 0.76\left(\mathrm{dt}, J=10.0,5.6 \mathrm{~Hz}, 1 \mathrm{H}, \mathrm{H}-1^{\prime}\right)$.

${ }^{13} \mathrm{C}$ NMR: $\delta_{\mathrm{C}}=171.1(\mathrm{CO}), 73.4(\mathrm{C}-5), 68.8(\mathrm{C}-3), 68.6(\mathrm{C}-4), 67.9(\mathrm{C}-6), 53.7(\mathrm{C}-1)$, $27.5\left(\mathrm{C}-{ }^{t} \mathrm{Bu}-\underline{\mathrm{CH}}_{3}\right), 27.4\left(\mathrm{C}-{ }^{t} \mathrm{Bu}-\underline{\mathrm{CH}}_{3}\right), 23.1\left(\mathrm{C}-{ }^{t} \mathrm{Bu}-\underline{\mathrm{CH}} 3\right), 20.6(\mathrm{C}-\mathrm{Ac}-\underline{\mathrm{CH}} 3), 12.0$ (C-2), $11.7\left(\mathrm{C} 1^{\prime}\right)$.

IR (neat): 2935, 2860, 1724, 1474, 1126, 1084, 825, $784 \mathrm{~cm}^{-1}$.

ES HRMS $m / z$ : calculated for $\mathrm{C}_{17} \mathrm{H}_{30} \mathrm{O}_{5} \mathrm{Si}+\mathrm{Na}$ : 365.1755. Found 365.1818. 


\section{6-Azido-2,2-di-tert-butyl-4a,6,7,9a-tetrahydro-(4H)-1,3,5-trioxa-2-silabenzocyclo heptene (3.5).}

To a solution of $3.4(70 \mathrm{mg}, 0.205 \mathrm{mmol})$ in $\mathrm{MeCN}(1.0 \mathrm{~mL})$ at $0{ }^{\circ} \mathrm{C}$ was added $\mathrm{TMSN}_{3}$ $(136 \mu \mathrm{L}, 1.03 \mathrm{mmol})$ then TMSOTf $(18 \mu \mathrm{L}, 0.103 \mathrm{mmol})$. The mixture was stirred for $4 \mathrm{~h}$ at $0{ }^{\circ} \mathrm{C}$ then poured onto saturated aqueous $\mathrm{NaHCO}_{3}(10 \mathrm{~mL})$, extracted with ether (2 $\times 20 \mathrm{~mL}$ ) and dried over $\mathrm{MgSO}_{4}$. Concentration in vacuo followed by flash chromatography on silica gel with hexanes:EtOAc (20:1) gave 3.5 as 2 epimers.

Major isomer: $\quad(4 \mathrm{a} R, 6 \mathrm{~S}, 9 \mathrm{a} R)-6$-Azido-2,2-di-tert-butyl-4a,6,7,9a-tetrahydro-(4H)1,3,5-trioxa-2-silabenzocycloheptene (3.5a).

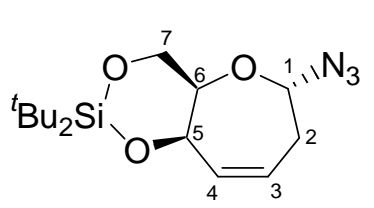

Clear oil (30mg, 46\%).

${ }^{1} \mathrm{H}$ NMR: $\delta_{\mathrm{H}}=5.71(\mathrm{ddd}, J=10.2,3.6,2.2 \mathrm{~Hz}, 1 \mathrm{H}, \mathrm{H}-4), 5.55$ (ddd, $J=12.2,4.1,1.0 \mathrm{~Hz}, 1 \mathrm{H}, \mathrm{H}-3), 5.43$ (dd, $J=9.3,4.4 \mathrm{~Hz}$, 1H, H-1), 4.86 (d, J=2.2 Hz, 1H, H, H-5), 4.32 (dd, J=11.9, $1.9 \mathrm{~Hz}, 1 \mathrm{H}, \mathrm{H}-7 \mathrm{a}), 4.20$ (dd, $J=11.9,2.2 \mathrm{~Hz}, 1 \mathrm{H}, \mathrm{H}-7 \mathrm{~b}), 4.12$ (dd, $J=2.2,1.9 \mathrm{~Hz}, 1 \mathrm{H}, \mathrm{H}-6$ ), 2.48 (ddd, $J=16.4$, 7.8, $4.4 \mathrm{~Hz}, 1 \mathrm{H}, \mathrm{H}-2 \mathrm{a}), 2.31(\mathrm{~m}, 1 \mathrm{H}, \mathrm{H}-2 \mathrm{~b}), 1.05$ (s, 9H, H- $\left.{ }^{t} \mathrm{Bu}-\underline{\mathrm{CH}}_{3}\right), 1.04(\mathrm{~s}, 9 \mathrm{H}, \mathrm{H}-$ $\left.{ }^{t} \mathrm{Bu}-\underline{\mathrm{CH}}_{3}\right)$.

${ }^{13} \mathrm{C}$ NMR: $\delta_{\mathrm{C}}=134.0(\mathrm{C}-4), 122.4$ (C-3), 90.1 (C-1), 74.7 (C-5), 70.0 (C-6), 68.1 (C-7), 31.0 (C-2), $27.5\left(\mathrm{C}-{ }^{t}{ }^{\mathrm{Bu}}-\underline{\mathrm{CH}}_{3}\right), 27.2\left(\mathrm{C}-{ }^{t} \mathrm{Bu}-\underline{\mathrm{CH}}_{3}\right), 23.2$ (Si-C), 20.7 (Si-C).

IR (neat): 2933, 2858, 2111, 1473, 1071, 825, $738 \mathrm{~cm}^{-1}$.

ES HRMS could not be obtained for this compound in either positive or negative modes.

Minor isomer: $\quad(4 \mathrm{a} R, 6 \boldsymbol{R}, 9 \mathrm{a} R)-6$-Azido-2,2-di-tert-butyl-4a,6,7,9a-tetrahydro-(4H)1,3,5-trioxa-2-sila-benzocycloheptene (3.5b).

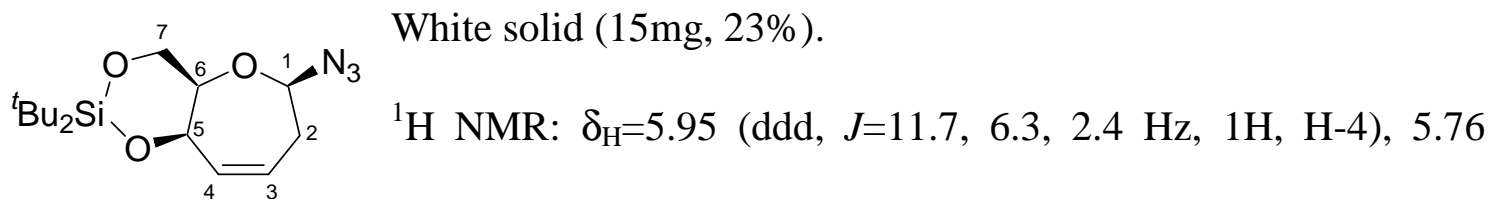
(ddd, $J=11.7,7.6,3.4 \mathrm{~Hz}, 1 \mathrm{H}, \mathrm{H}-3), 4.83$ (dd, $J=9.3,1.9 \mathrm{~Hz}, 1 \mathrm{H}, \mathrm{H}-1$ ), 4.74 (dd, $J=6.6$, 
$1.7 \mathrm{~Hz}, 1 \mathrm{H}, \mathrm{H}-5), 4.27$ (t, J=2.2 Hz, 2H, H-7) 3.71 (dd, J=3.3, $2.2 \mathrm{~Hz}, 1 \mathrm{H}, \mathrm{H}-6), 2.68$ (m, 1H, H-2a), 2.42 (ddd, $J=16.8,7.7,2.2 \mathrm{~Hz}, 1 \mathrm{H}, \mathrm{H}-2 \mathrm{~b}$ ), 1.07 (s, 9H, H- ${ }^{t} \mathrm{Bu}^{-\mathrm{CH}_{3}}$ ), 1.05 (s, $\left.9 \mathrm{H}, \mathrm{H}-{ }^{t} \mathrm{Bu}-\underline{\mathrm{CH}}_{3}\right)$.

${ }^{13} \mathrm{C}$ NMR: $\delta_{\mathrm{C}}=131.6(\mathrm{C}-4), 127.2(\mathrm{C}-3), 90.6(\mathrm{C}-1), 77.3$ (C-6), 72.9 (C-5), $68.3(\mathrm{C}-7)$, $36.1(\mathrm{C}-2), 27.5\left(\mathrm{C}-{ }^{t} \mathrm{Bu}-\underline{\mathrm{CH}}_{3}\right), 27.4\left(\mathrm{C}-{ }^{t} \mathrm{Bu}-\underline{\mathrm{CH}}_{3}\right), 23.1$ (Si-C), 21.1 (Si-C).

${ }^{15} \mathrm{~N}$ NMR: $\delta_{\mathrm{N}}=-300.1,-288.4,-135.8$.

IR (neat): 2933, 2859, 2108, 1473, 1246, 1117, 884, 825, $780 \mathrm{~cm}^{-1}$.

ES HRMS could not be obtained for this compound in either positive or negative modes.

(4a,6,9a)-2,2-Di-tert-butyl-6-phenylsulfanyl-4a,6,7,9a-tetrahydro-(4H)-1,3,5-trioxa2-sila-benzocycloheptene (3.6).

To a solution of $3.4(120 \mathrm{mg}, 0.35 \mathrm{mmol})$ in $\mathrm{MeCN}(1.0 \mathrm{~mL})$ at $0{ }^{\circ} \mathrm{C}$ was added TMSSPh $(330 \mu \mathrm{L}, 1.75 \mathrm{mmol})$ followed by TMSOTf $(15 \mu \mathrm{L}, 0.075 \mathrm{mmol})$. The solution was stirred for $30 \mathrm{~min}$ at $0{ }^{\circ} \mathrm{C}$ then $5 \mathrm{~h}$ warming to ambient temperature. The mixture was poured onto saturated aq $\mathrm{NaHCO}_{3}(20 \mathrm{~mL})$, extracted with $\mathrm{Et}_{2} \mathrm{O}(2 \times 20$ $\mathrm{mL}$ ) and dried over $\mathrm{MgSO}_{4}$. Concentration in vacuo followed by flash chromatography on silica gel with hexanes:EtOAc (20:1) gave $\mathbf{3 . 6}$ as a colourless oil (117 mg, 85\% yield) as a 6:1 mixture of epimers by GC.

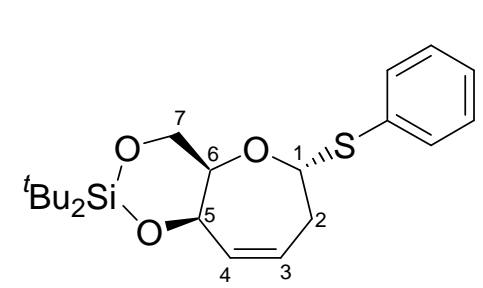

Major isomer $(4 \mathbf{a} \boldsymbol{R}, \mathbf{6} \boldsymbol{R}, 9 \mathrm{a} \boldsymbol{R}) .{ }^{1} \mathrm{H}$ NMR: $\delta_{\mathrm{H}}=7.55(\mathrm{~m}, 2 \mathrm{H}, \mathrm{H}-$ Ar), 7.30 (m, 3H, H-Ar), 5.75 (m, 2H, H-3, H-4), 5.66 (dd, $J=9.3,5.9 \mathrm{~Hz}, 1 \mathrm{H}, \mathrm{H}-1), 4.95$ (s, 1H, H-5), 4.30 (dd, $J=12.0$, $2.2 \mathrm{~Hz}, 1 \mathrm{H}, \mathrm{H}-7 \mathrm{a}), 4.26$ (dd, J=4.4, $2.2 \mathrm{~Hz}, 1 \mathrm{H}, \mathrm{H}-6), 4.12$ (dd, J=12.0, $2.4 \mathrm{~Hz}, 1 \mathrm{H}, \mathrm{H}-$ 7b), 2.69 (m, 2H, H-H-2), 1.07 (s, 9H, H- $\left.{ }^{t}{ }^{\mathrm{Bu}}-\underline{\mathrm{CH}}_{3}\right), 1.06$ (s, 9H, H- $\left.{ }^{t} \mathrm{Bu}-\underline{\mathrm{CH}}_{3}\right)$. 
${ }^{13} \mathrm{C}$ NMR: $\delta_{\mathrm{C}}=133.6(\mathrm{C}-4), 130.1(\mathrm{C}-\mathrm{Ar}), 128.7(\mathrm{C}-\mathrm{Ar}), 126.5(\mathrm{C}-\mathrm{Ar}), 123.9(\mathrm{C}-3)$, 86.2 (C-1), 75.4 (C-5), 68.8 (C-6), 68.3 (C-7), 32.2 (C-2), $27.5\left(\mathrm{C}-t \mathrm{Bu}-\underline{\mathrm{CH}}_{3}\right), 27.4$ (C$\left.{ }^{t} \mathrm{Bu}-\underline{\mathrm{CH}}_{3}\right), 27.3\left(\mathrm{C}-{ }^{t} \mathrm{Bu}-\underline{\mathrm{CH}}_{3}\right), 27.2\left(\mathrm{C}-{ }^{t} \mathrm{Bu}-\underline{\mathrm{CH}}_{3}\right), 23.5$ (Si-C), 20.8 (Si-C).

IR (neat): 2934, 2858, 1473, 1140, 1067, 824, $729 \mathrm{~cm}^{-1}$.

ES HRMS m/z: calculated for $\mathrm{C}_{21} \mathrm{H}_{32} \mathrm{O}_{3} \mathrm{Si}+\mathrm{H}$ : $(\mathrm{M}+\mathrm{H})$ 393.1911. Found 393.1914.

(4aR,6R,9aR)-6-Allyl-2,2-di-tert-butyl-4a,6,7,9a-tetrahydro-(4H)-1,3,5-trioxa-2silabenzocycloheptane (3.7).

To a solution of $3.4(120 \mathrm{mg}, 0.35 \mathrm{mmol})$ in $\mathrm{MeCN}(1 \mathrm{~mL})$ at $-20{ }^{\circ} \mathrm{C}$ was added allyltrimethylsilane $(280 \mu \mathrm{L}, 1.75 \mathrm{mmol})$ followed by TMSOTf $(15 \mu \mathrm{L}, 0.087 \mathrm{mmol})$. The solution was stirred for 30 min allowing it to warm to $0{ }^{\circ} \mathrm{C}$. After stirring an additional $30 \mathrm{~min}$ at $0{ }^{\circ} \mathrm{C}$, the solution was poured onto saturated aq $\mathrm{NaHCO}_{3}(10 \mathrm{~mL})$, extracted with $\mathrm{Et}_{2} \mathrm{O}(2 \times 20 \mathrm{~mL})$ and dried over $\mathrm{MgSO}_{4}$. Concentration in vacuo followed by flash chromatography on silica gel with hexanes:EtOAc (20:1) gave 3.7 as a colourless oil (113 $\mathrm{mg}, 76 \%)$ as an 80:1 mix of epimers by GC.

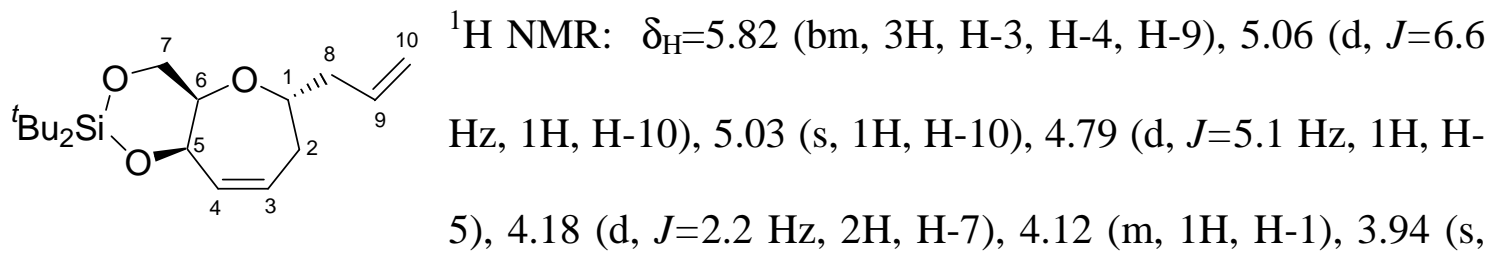

1H, H-6), 2.25 (m, 4H, H-2, H-8), 1.05 (s, 9H, H- $\left.{ }^{t} \mathrm{Bu}-\underline{C H}_{3}\right), 1.04$ (s, 9H, H- $\left.{ }^{t} \mathrm{Bu}-\underline{C H}_{3}\right)$.

${ }^{13} \mathrm{C}$ NMR: $\delta_{\mathrm{C}}=135.1(\mathrm{C}-9), 131.9(\mathrm{C}-4), 130.7(\mathrm{C}-3), 117.3(\mathrm{C}-10), 76.5(\mathrm{C}-1), 74.3(\mathrm{C}-$ 5), 72.7 (C-6), 69.8 (C-7), 41.1 (C-8), 32.1 (C-2), $28.0\left(\mathrm{C}-{ }^{t} \mathrm{Bu}-\underline{\mathrm{CH}}_{3}\right), 27.6\left(\mathrm{C}-{ }^{t}{ }^{\mathrm{Bu}}-\underline{\mathrm{CH}}_{3}\right)$, 23.3 (Si-C), $21.0(\mathrm{Si}-\mathrm{C})$.

IR (neat): 2934, 2865, 1473, 1085, 907, 825, $730 \mathrm{~cm}^{-1}$.

ES HRMS m/z: calculated for $\mathrm{C}_{18} \mathrm{H}_{32} \mathrm{O}_{3} \mathrm{Si}+\mathrm{H}$ : 325.2194. Found 325.2207. 
(4aR,6R,9a $R)-2,2-D i-t e r t-b u t y l-6-p r o p a-1,2-d i e n y l-4 a, 6,7,9 a-t e t r a h y d r o-(4 H)-1,3,5-$ trioxa-2-silabenzocycloheptane (3.8).

To a solution of $3.4(70 \mathrm{mg}, 0.205 \mathrm{mmol})$ in $\mathrm{MeCN}(1.0 \mathrm{~mL})$ at $0{ }^{\circ} \mathrm{C}$ was added 2propynylTMS (153 $\mu \mathrm{L}, 1.08 \mathrm{mmol})$ followed by TMSOTf $(18 \mu \mathrm{L}, 0.121 \mathrm{mmol})$. The mixture was stirred at $0{ }^{\circ} \mathrm{C}$ for $1 \mathrm{~h}$ then poured onto saturated aq $\mathrm{NaHCO}_{3}(20 \mathrm{~mL})$, extracted with ether $(3 \times 20 \mathrm{~mL})$ and dried over $\mathrm{MgSO}_{4}$. Column chromatography on silica gel with hexanes:EtOAc (20:1) gave 3.7 as a colourless oil (45 mg, 68\%).

${ }^{1} \mathrm{H}$ NMR: $\delta_{\mathrm{H}}=5.7(\mathrm{ddd}, J=11.0,7.3,3.4 \mathrm{~Hz}, 1 \mathrm{H}, \mathrm{H}-3), 5.78$

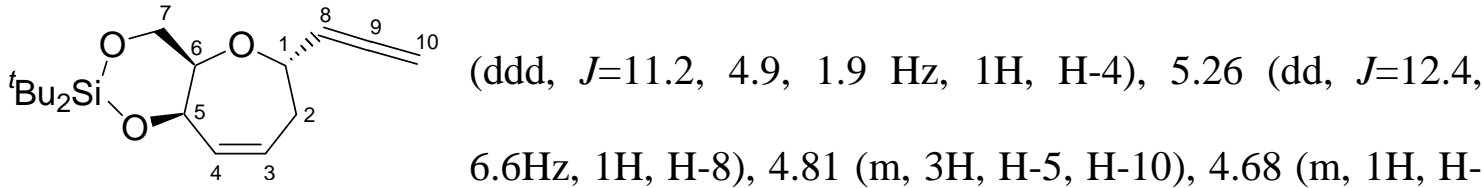
1), 4.19 (dd, J=4.4, 2.2 Hz, 2H, H-7), 3.95 (d, $J=1.9 \mathrm{~Hz}, 1 \mathrm{H}, \mathrm{H}-6), 2.6-2.3$ (bm, 2H, H2), $1.06\left(\mathrm{~s}, 9 \mathrm{H}, \mathrm{H}-{ }^{t} \mathrm{Bu}-\underline{\mathrm{CH}}_{3}\right), 1.05\left(\mathrm{~s}, 9 \mathrm{H}, \mathrm{H}-{ }^{t} \mathrm{Bu}-\underline{\mathrm{CH}}_{3}\right)$

${ }^{13} \mathrm{C}$ NMR: $\delta_{\mathrm{C}}=207.7(\mathrm{C}-9), 132.0(\mathrm{C}-4), 129.0(\mathrm{C}-3), 92.6$ (C-8), $77.0(\mathrm{C}-10), 74.3(\mathrm{C}-$ 1), 74.2 (C-5), 71.7 (C-6), 69.3 (C-7), $32.2(\mathrm{C}-2), 27.7\left(\mathrm{C}-t \mathrm{Bu}-\underline{\mathrm{CH}}_{3}\right), 27.6\left(\mathrm{C}-t \mathrm{Bu}-\underline{\mathrm{CH}}_{3}\right)$, $27.5\left(\mathrm{C}-{ }^{t} \mathrm{Bu}-\underline{\mathrm{CH}}_{3}\right), 27.3\left(\mathrm{C}-{ }^{t} \mathrm{Bu}-\underline{\mathrm{CH}}_{3}\right), 27.2\left(\mathrm{C}-{ }^{t} \mathrm{Bu}-\underline{\mathrm{CH}}_{3}\right), 23.1$ (Si-C), 20.8 (Si-C). IR (neat): $3312,2933,2859,1954,1472,1077,908,839,733 \mathrm{~cm}^{-1}$

(4aR,6S,9aR)-2,2-Di-tert-butyl-4a,6,7,9a-tetrahydro-(4H)-1,3,5-trioxa-2-silabenzo cyclohepten-6-yl)-acetic acid ethyl ester (3.11).

To a solution of $3.4(57 \mathrm{mg}, 0.167 \mathrm{mmol})$ in $\mathrm{MeCN}(1.0 \mathrm{~mL})$ at $0{ }^{\circ} \mathrm{C}$ was added 1 (trimethylsilyl)oxy-1-ethoxyethene $\mathbf{3 . 9}$ (181 mg, $1.17 \mathrm{mmol}$ ) followed by TMSOTf (15 $\mu \mathrm{L}, 0.083 \mathrm{mmol})$. The solution was allowed to warm to room temperature and stirred for $4 \mathrm{~h}$ then poured onto saturated aq $\mathrm{NaHCO}_{3}(10 \mathrm{~mL})$, extracted with $\mathrm{Et}_{2} \mathrm{O}(2 \times 20$ $\mathrm{mL}$ ) and dried over $\mathrm{MgSO}_{4}$. Concentration in vacuo followed by flash chromatography on silica gel with hexanes:EtOAc (20:1) gave $\mathbf{3 . 1 1}$ as a colourless oil (38 $\mathrm{mg}, 68 \%)$ as a 3:1 mixture of epimers by GC. 


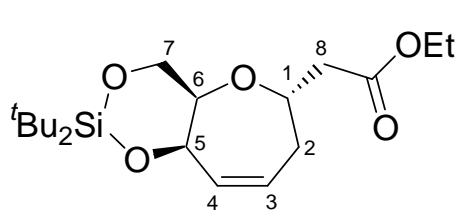

${ }^{1} \mathrm{H}$ NMR: $\delta_{\mathrm{H}}=5.86-5.75(\mathrm{~m}, 2 \mathrm{H}, \mathrm{H}-3, \mathrm{H}-4), 4.83(\mathrm{~m})$ and $4.78(\mathrm{dd}, J=5.9,1.9 \mathrm{~Hz})(1 \mathrm{H}, \mathrm{H}-5), 4.46(\mathrm{~m}, 1 \mathrm{H}, \mathrm{H}-1)$, 4.19-4.08 (m, 4H, H-7, O- $\left.\underline{\mathrm{CH}}_{2}-\mathrm{CH}_{3}\right), 3.99$ (dd, $J=4.5,2.5$ $\mathrm{Hz})$ and $3.67(\mathrm{dd}, J=5.4,2.4 \mathrm{~Hz})(1 \mathrm{H}, \mathrm{H}-6), 2.60-2.39$ (m, 3H, H-2a, H-8), 2.30-2.22 (m, 1H, H-2b), 1.25 (q, J=6.1 Hz, 3H, O- $\left.\mathrm{CH}_{2}-\underline{\mathrm{CH}}_{3}\right), 1.04$ (s, 9H, H- $\left.{ }^{t}{ }^{\mathrm{Bu}}-\underline{\mathrm{CH}}_{3}\right), 1.03$ (s, $\left.9 \mathrm{H}, \mathrm{H}-{ }^{t} \mathrm{Bu}-\underline{\mathrm{CH}}_{3}\right)$.

${ }^{13} \mathrm{C}$ NMR: major isomer: $\delta_{\mathrm{C}}=171.0(\mathrm{C}-9), 132.4(\mathrm{C}-4), 128.4(\mathrm{C}-3), 74.4(\mathrm{C}-5), 73.5$ (C-1), 71.8 (C-6), 68.7 (C-7), $60.4\left(\mathrm{O}-\underline{\mathrm{CH}}_{2}-\mathrm{CH}_{3}\right), 41.5(\mathrm{C}-8), 32.2(\mathrm{C}-2), 27.6\left(\mathrm{H}-{ }^{t} \mathrm{Bu}-\right.$ $\left.\underline{\mathrm{CH}}_{3}\right), 27.3\left(\mathrm{H}_{-}{ }^{t} \mathrm{Bu}-\underline{\mathrm{CH}}_{3}\right), 22.9(\mathrm{Si}-\mathrm{C}), 20.8(\mathrm{Si}-\mathrm{C}), 14.2\left(\mathrm{O}-\mathrm{CH}_{2}-\underline{\mathrm{CH}}_{3}\right)$; minor isomer: $\delta_{\mathrm{C}}=171.3(\mathrm{C}-9), 130.9(\mathrm{C}-4), 129.9(\mathrm{C}-3), 78.6(\mathrm{C}-5), 76.0(\mathrm{C}-1), 73.6(\mathrm{C}-6), 68.4(\mathrm{C}-$ 7), $60.4\left(\mathrm{Et}^{-\mathrm{CH}_{2}-}\right), 41.9$ (C-8), $36.3(\mathrm{C}-2), 27.4\left(\mathrm{C}-{ }^{t} \mathrm{Bu}-\underline{\mathrm{CH}}_{3}\right), 27.2\left(\mathrm{C}-{ }^{t} \mathrm{Bu}-\underline{\mathrm{CH}}_{3}\right), 21.1$ (Si-C), 14.2 (Et-Me).

IR (neat): 2934, 2858, 1731, 1473, 1095, 825, 776, 732 $\mathrm{cm}^{-1}$.

\section{2-((4aR,6S,9aR)-2,2-Di-tert-butyl-4a,6,7,9a-tetrahydro-(4H)-1,3,5-trioxa-2-sila benzocyclohepten-6-yl)-2-methylpropionic acid ethyl ester (3.12).}

To a solution of $3.4(70 \mathrm{mg}, 0.205 \mathrm{mmol})$ in $\mathrm{MeCN}(1.0 \mathrm{~mL})$ at $0{ }^{\circ} \mathrm{C}$ was added of 1 (trimethylsilyl)oxy-1-ethoxy-2-methyl-1-propene $\mathbf{3 . 1 0}$ (188 mg, $1.03 \mathrm{mmol}$ ) followed by TMSOTf $(18 \mu \mathrm{L}, 0.134 \mathrm{mmol})$. The solution was allowed to warm to ambient temperature and stirred overnight then was poured onto saturated aq $\mathrm{NaHCO}_{3}(10 \mathrm{~mL})$, extracted with $\mathrm{Et}_{2} \mathrm{O}(3 \times 20 \mathrm{~mL})$ and dried over $\mathrm{MgSO}_{4}$. Flash chromatography on silica gel with hexanes:EtOAc (20:1) gave $\mathbf{3 . 1 2}$ as a colourless oil (63 mg 77\%).<smiles>CCOC(=O)C(C)(C)[C@H]1CC=C[C@@H]2O[SiH](C(C)(C)C)OC[C@H]2O1</smiles>

${ }^{1} \mathrm{H}$ NMR: $\delta_{\mathrm{H}}=5.82-5.65(\mathrm{~m}, 2 \mathrm{H}, \mathrm{H}-3, \mathrm{H}-4), 4.80(\mathrm{~d}, J=1.7$ $\mathrm{Hz}, 1 \mathrm{H}, \mathrm{H}-5), 4.25-4.01$ (m, 6H, H-1, H-6, H-7, O- $\underline{\mathrm{H}}_{2}-$ $\left.\mathrm{CH}_{3}\right), 2.51$ (tdd, $\left.J=14.7,4.5,2.7 \mathrm{~Hz}, 1 \mathrm{H}, \mathrm{H}-2 \mathrm{a}\right), 1.97$ (ddd, 
$J=16.4,8.8,1.9 \mathrm{~Hz}, 1 \mathrm{H}, \mathrm{H}-2 \mathrm{~b}), 1.23\left(\mathrm{t}, J=7.1 \mathrm{~Hz}, 3 \mathrm{H}, \mathrm{O}-\mathrm{CH}_{2}-\underline{\mathrm{CH}}_{3}\right), 1.16$ (s, 3H, H-13), $1.11(\mathrm{~s}, 3 \mathrm{H}, \mathrm{H}-14), 1.01$ (d, $\left.J=1 \mathrm{~Hz}, 18 \mathrm{H}, \mathrm{H}^{t}{ }^{t} \mathrm{Bu}-\underline{\mathrm{CH}}_{3}\right)$.

${ }^{13} \mathrm{C}$ NMR: $\delta_{\mathrm{C}}=176.5$ (C-11), $132.7(\mathrm{C}-4), 126.8$ (C-3), 81.3 (C-1), 75.3 (C-5), 71.9 (C6), 68.2 (C-7), $60.4\left(\mathrm{O}-\underline{\mathrm{CH}}_{2}-\mathrm{CH}_{3}\right), 48.0(\mathrm{C}-11), 27.5\left(\mathrm{C}-{ }^{t} \mathrm{Bu}-\underline{\mathrm{CH}}_{3}\right), 27.3\left(\mathrm{C}-{ }^{t} \mathrm{Bu}-\underline{\mathrm{CH}}_{3}\right)$, 26.1 (C-2), 22.9 (C-9), 22.0 (C-10), 20.7 (Si-C), 20.5 (Si-C), $14.1\left(\mathrm{O}-\mathrm{CH}_{2}-\mathrm{CH}_{3}\right)$.

IR (neat): 2934, 2859, 1724, 1473, 1263, 1099, 910, 825, 775, $732 \mathrm{~cm}^{-1}$.

ES HRMS $m / z$ : calculated for $\mathrm{C}_{21} \mathrm{H}_{38} \mathrm{O}_{5} \mathrm{Si}+\mathrm{H}: 399.2561$. Found 399.2582.

\section{$(1 S, 3 R, 4 S, 5 R, 6 S)-3$-(tert-Butyldiphenylsilanyloxymethyl)-2-oxa-bicyclo[4.1.0]}

\section{heptane-4,5-diol. (3.15).}

To a solution of $\mathbf{3 . 1 4}(1.7 \mathrm{~g}, 4.427 \mathrm{mmol})$ in $\mathrm{Et}_{2} \mathrm{O}(20 \mathrm{~mL})$ was added $\mathrm{CH}_{2} \mathrm{I}_{2}(1.78 \mathrm{~mL}$, $22.135 \mathrm{mmol})$ and $\mathrm{Et}_{2} \mathrm{Zn}(1 \mathrm{M}, 22.1 \mathrm{~mL}, 22.1 \mathrm{mmol})$. The mixture was refluxed for 12 $\mathrm{h}$ and was then treated with a further five equivalents of $\mathrm{Et}_{2} \mathrm{Zn}$ and $\mathrm{CH}_{2} \mathrm{I}_{2}$, then refluxed until the reaction was complete by ${ }^{1} \mathrm{H}$ NMR. The solution was poured onto saturated aq $\mathrm{NH}_{4} \mathrm{Cl}(100 \mathrm{~mL})$ and extracted with $\mathrm{Et}_{2} \mathrm{O}(2 \times 75 \mathrm{~mL})$. The combined ether layers were washed with $\mathrm{H}_{2} \mathrm{O}(50 \mathrm{~mL})$, saturated brine $(50 \mathrm{~mL})$ and dried over $\mathrm{MgSO}_{4}$. Concentration in vacuo followed by flash chromatography on silica gel with hexanes:EtOAc (1:1) gave $\mathbf{3 . 1 5}$ as a white solid (1.34g, 76\%).

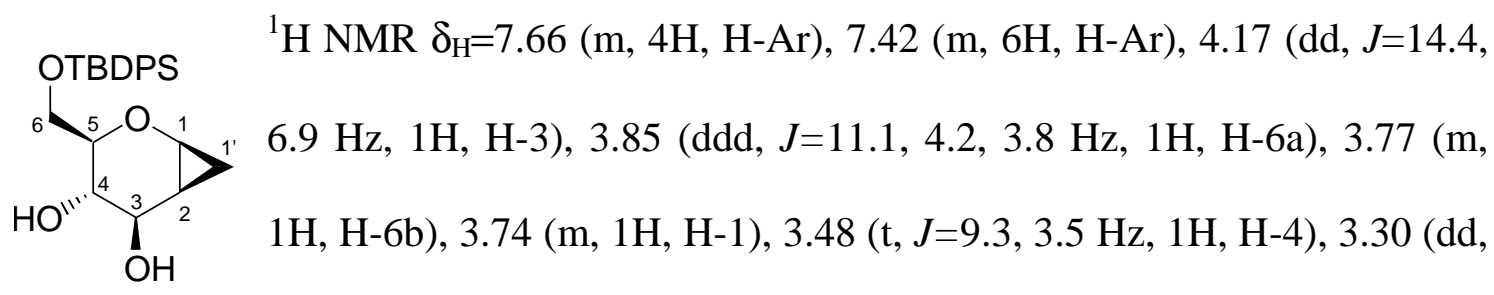
$J=9.5,4.5 \mathrm{~Hz}, 1 \mathrm{H}, \mathrm{H}-5), 2.93$ (s, 1H, -OH), 2.30 (s, 1H, -OH), 1.42 (m, 1H, H-2), 1.07 (s, 9H, H- $\left.{ }^{t} \mathrm{Bu}-\underline{\mathrm{CH}}_{3}\right), 0.72$ (dd, J=8.4, $4.5 \mathrm{~Hz}, 2 \mathrm{H}, \mathrm{H}-{ }^{\prime}$ ').

${ }^{13} \mathrm{C} \delta_{\mathrm{C}}=135.6(\mathrm{C}-\mathrm{Ar}), 132.6(\mathrm{C}-\mathrm{Ar}), 129.9(\mathrm{C}-\mathrm{Ar}), 127.8$ (C-Ar), $77.2(\mathrm{C}-5), 74.1(\mathrm{C}-4)$, 71.9 (C-3), 64.6 (C-6), 53.9 (C-1), $26.8\left(\mathrm{C}^{-}{ }^{t} \mathrm{Bu}-\underline{\mathrm{CH}}_{3}\right), 19.2$ (Si-C), 17.9 (C-2), 11.9 C-1'). 
ES HRMS m/z: calculated for $\mathrm{C}_{23} \mathrm{H}_{30} \mathrm{O}_{4} \mathrm{Si}+\mathrm{NH}_{4}$ 416.2252. Found 416.2252.

MP: $123{ }^{\circ} \mathrm{C}$.

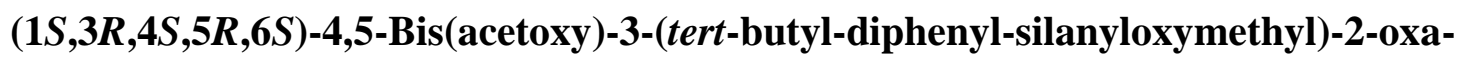
bicyclo[4.1.0]heptane-4,5-diol. (3.16).

To a solution of $\mathbf{3 . 1 5}(1.6 \mathrm{~g}, 4.0 \mathrm{mmol})$ in $\mathrm{CH}_{2} \mathrm{Cl}_{2}(40 \mathrm{~mL})$ with a catalytic amount of DMAP at ambient temperature was added acetic anhydride $(1.52 \mathrm{~mL}, 16 \mathrm{mmol})$ and pyridine (1.94 $\mathrm{mL}, 24 \mathrm{mmol})$, and the reaction stirred for $12 \mathrm{~h}$. The mixture was then poured onto $\mathrm{CH}_{2} \mathrm{Cl}_{2}(50 \mathrm{~mL})$, washed with $\mathrm{H}_{2} \mathrm{O}(4 \times 10 \mathrm{~mL})$, and dried over $\mathrm{MgSO}_{4}$. Concentration in vacuo followed by flash chromatography on silica gel with hexanes:EtOAc (5:1) gave 3.16 as a colourless oil $(1.857 \mathrm{~g}, 96 \%)$.

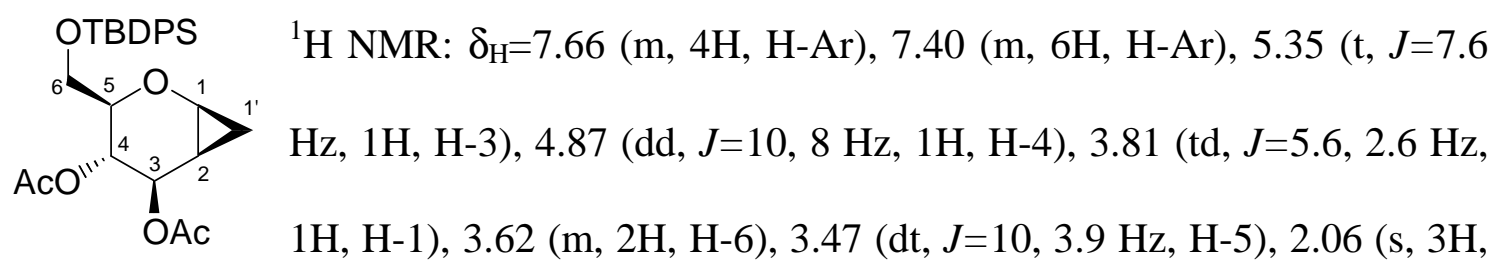
H-Ac), 1.88 (s, 3H, H-Ac), 1.53 (m, 1H, H-2), 1.04 (s, 9H, H- $\left.{ }^{t}{ }^{\mathrm{Bu}}-\underline{\mathrm{CH}}_{3}\right), 0.86$ (td, J=6.6, $2.7 \mathrm{~Hz}, 1 \mathrm{H}, \mathrm{H}-1^{\prime}$ ), 0.77 (dt, $J=9.8,5.8 \mathrm{~Hz}, 1 \mathrm{H}, \mathrm{H}-1^{\prime}$ ).

${ }^{13} \mathrm{C}$ NMR: $\delta_{\mathrm{C}}=171.1(\mathrm{CO}), 169.2(\mathrm{CO}), 135.7(\mathrm{C}-\mathrm{Ar}), 135.6(\mathrm{C}-\mathrm{Ar}), 133.4(\mathrm{C}-\mathrm{Ar})$, 133.3 (C-Ar), 129.62 (C-Ar), 129.61 (C-Ar), 127.6 (C-Ar), 127.5 (C-Ar), 77.7 (C-5), 72.5 (C-3), 69.9 (C-4), 62.9 (C-6), $54.3(\mathrm{C}-1), 26.7\left(\mathrm{C}-{ }^{t} \mathrm{Bu}-\underline{\mathrm{CH}}_{3}\right), 21.1\left(\mathrm{C}-\mathrm{Ac}-\underline{\mathrm{CH}}_{3}\right), 20.7$ (C-Ac- $\left.\underline{\mathrm{CH}}_{3}\right), 19.2$ (Si-C), 16.0 (C-2), 12.5 (C-1').

ES HRMS $m / z$ : calculated for $\mathrm{C}_{27} \mathrm{H}_{34} \mathrm{O}_{6} \mathrm{Si}+\mathrm{NH}_{4}$. 500.2463. Found 500.2468. IR: $2932,2858,1749,1714,1428,1370,1218,1110,822,741,702 \mathrm{~cm}^{-1}$.

(2R,3S,7S)-3-Acetoxy-7-azido-2-(tert-butyldipenylsilanyloxymethyl)-2,3,6,7tetrahydro-oxepin-4-yl (3.17). 
To a solution of $\mathbf{3 . 1 6}(99 \mathrm{mg}, 0.205 \mathrm{mmol})$ in $\mathrm{MeCN}(1 \mathrm{~mL})$ at $0{ }^{\circ} \mathrm{C}$ was added $\mathrm{TMSN}_{3}$ $(136 \mu \mathrm{L}, 1.03 \mathrm{mmol})$ and TMSOTf $(18 \mu \mathrm{L}, 0.103 \mathrm{mmol})$. The mixture was stirred for $30 \mathrm{~min}$ at $0{ }^{\circ} \mathrm{C}$ and then warmed to ambient temperature and stirred for $1 \mathrm{~h}$. The mixture was poured onto saturated aq $\mathrm{NaHCO}_{3}(10 \mathrm{~mL})$, extracted with $\mathrm{Et}_{2} \mathrm{O}(2 \mathrm{x}$ 20mL), dried over $\mathrm{MgSO}_{4}$ and concentrated in vacuo. Flash chromatography on silica gel with hexanes:EtOAc (20:1) gave $\mathbf{3 . 1 7}$ as a clear oil $(59 \mathrm{mg}, 61 \%)$ in a 2.5:1 ratio of isomers by GC.

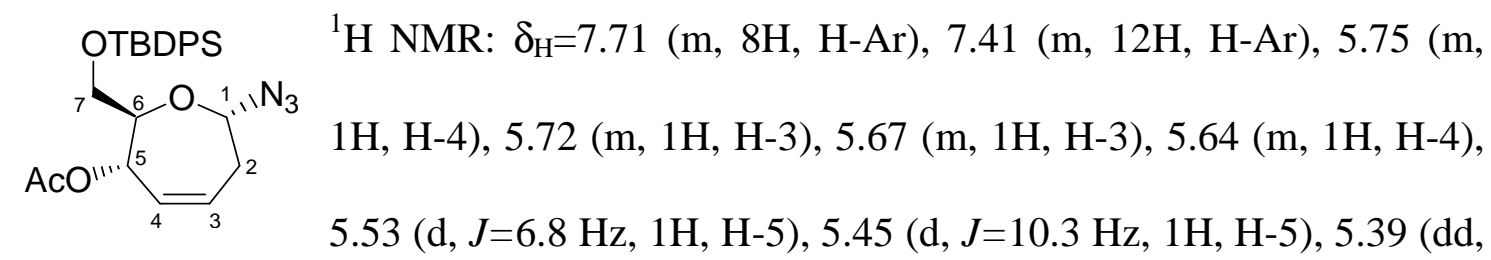
$J=8.8,2.9 \mathrm{~Hz}, 1 \mathrm{H}, \mathrm{H}-1), 4.94(\mathrm{dd}, J=10.1,4.9 \mathrm{~Hz}, 1 \mathrm{H}, \mathrm{H}-1), 4.29$ (dt, $J=10.2,3.4 \mathrm{~Hz}$, 1H, H-6), 3.81 (m, 1H, H-6), 3.76 (m, 4H, H-7), 2.35-2.65 (bm, 4H, H-2), 1.97 (s, 3H, H-Ac), 1.91 (s, 3H, H-Ac), 1.08 (s, 9H, H- $\left.{ }^{t} \mathrm{Bu}-\underline{\mathrm{CH}}_{3}\right), 1.06$ (s, 9H, H- $\left.{ }^{t} \mathrm{Bu}-\underline{\mathrm{CH}}_{3}\right)$.

${ }^{13} \mathrm{C}$ NMR: $\delta_{\mathrm{C}}=169.6(\mathrm{C}-\mathrm{Ac}), 135.7(\mathrm{C}-\mathrm{Ar}), 135.5$ (C-Ar), $135.1(\mathrm{C}-\mathrm{Ar}), 134.7(\mathrm{C}-\mathrm{Ar})$, 133.1 (C-Ar), 132.0 (C-4), 129.6 (C-Ar), 129.4 (C-4), 127.7 (C-Ar), 125.1 (C-3), 125.0 (C-3), 90.5 (C-1), 89.2 (C-1), 81.1 (C-6), 72.2 (C-6), 71.1 (C-5), 70.2 (C-5), 64.2 (C-7), 64.0 (C-7), $36.0(\mathrm{C}-2), 31.4(\mathrm{C}-2), 26.6\left(\mathrm{C}-{ }^{t} \mathrm{Bu}-\underline{\mathrm{CH}}_{3}\right), 26.5\left(\mathrm{C}-{ }^{t} \mathrm{Bu}-\underline{\mathrm{CH}}_{3}\right), 20.9(\mathrm{C}-\mathrm{Ac}-$ $\left.\underline{\mathrm{CH}}_{3}\right), 19.1$ (Si-C), 19.0 (Si-C).

\section{(2R,3S,7S)-3-Acetoxy-7-phenylsulfanyl-2-(tert-butyldipenylsilanyloxymethyl)-}

2,3,6,7-tetrahydro-oxepin-4-yl (3.18).

To a solution of $\mathbf{3 . 1 6}(97 \mathrm{mg}, 0.2 \mathrm{mmol})$ in $\mathrm{MeCN}(1 \mathrm{~mL})$ at $0{ }^{\circ} \mathrm{C}$ was added TMSSPh (190 uL, $1 \mathrm{mmol})$ and TMSOTf (17 uL, $0.1 \mathrm{mmol})$, the mixture stirred for $30 \mathrm{~min}$ and then allowed to warm to ambient temperature. The mixture was poured onto saturated aq $\mathrm{NaHCO}_{3}(10 \mathrm{~mL})$, extracted with $\mathrm{Et}_{2} \mathrm{O}(2 \times 20 \mathrm{~mL})$ and the organic layers dried over 
$\mathrm{MgSO}_{4}$ and concentrated in vacuo. Flash chromatography on silica gel with hexanes:EtOAc (20:1) gave $\mathbf{3 . 1 8}$ as a clear oil (46 mg, 45\%) in a 6:1 ratio of isomers.

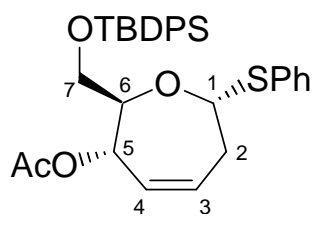

${ }^{1} \mathrm{H}$ NMR: $\delta_{\mathrm{H}}=7.66(\mathrm{~m}, 4 \mathrm{H}, \mathrm{H}-\mathrm{Ar}), 7.51(\mathrm{~m}, 2 \mathrm{H}, \mathrm{H}-\mathrm{Ar}), 7.39$ (m, 6H, H-Ar), 7.21 (m, 3H, H-Ar), 5.83 (s, 2H, H-3, H-4), 5.60 (m, 1H, H5), $5.53(\mathrm{dd}, J=9.8,6.1 \mathrm{~Hz}, 1 \mathrm{H}, \mathrm{H}-1), 4.39$ (dt, $J=9.7,3.4 \mathrm{~Hz}, 1 \mathrm{H}$, H-6), 3.75 (m, 2H, H-7), 2.75 (m, 2H, H-2), 1.96 (s, 3H, H-Ac- $\left.\underline{\mathrm{CH}}_{3}\right), 1.03$ (s, 9H, H$\left.{ }^{t} \mathrm{Bu}-\underline{\mathrm{CH}}_{3}\right)$.

${ }^{13} \mathrm{C}$ NMR: $\delta_{\mathrm{C}}=169.7(\mathrm{CO}), 135.7(\mathrm{C}-\mathrm{Ar}), 130.4(\mathrm{C}-4), 129.7(\mathrm{C}-\mathrm{Ar}), 128.6(\mathrm{C}-\mathrm{Ar})$, 127.7 (C-Ar), 126.9 (C-Ar), 126.3 (C-3), 85.1 (C-1), 71.0 (C-5), 70.9 (C-6), 64.5 (C-7), $32.6(\mathrm{C}-2), 26.7\left(\mathrm{C}-{ }^{t} \mathrm{Bu}-\underline{\mathrm{CH}}_{3}\right), 20.9(\mathrm{Si}-\mathrm{C}), 19.2\left(\mathrm{C}-\mathrm{Ac}-\underline{\mathrm{CH}}_{3}\right)$.

ES HRMS $m / z$ : calculated for $\mathrm{C}_{31} \mathrm{H}_{36} \mathrm{O}_{4} \mathrm{SSi}+\mathrm{NH}_{4}$ 550.2442. Found 550.2422.

\section{(2R,3S,7S)-3-Acetoxy-7-propa-1,2-dienyl-2-(tert-butyldipenylsilanyloxymethyl)-}

\section{2,3,6,7-tetrahydro-oxepin-4-yl (3.19).}

To a solution of $3.16(84 \mathrm{mg}, 0.174 \mathrm{mmol})$ in $\mathrm{MeCN}(1 \mathrm{~mL})$ at $0{ }^{\circ} \mathrm{C}$ was added 2propynyltrimethylsilane $(129 \mu \mathrm{L}, 0.87 \mathrm{mmol})$ and TMSOTf $(15 \mu \mathrm{L}, 0.087 \mathrm{mmol})$. The reaction was stirred at $0{ }^{\circ} \mathrm{C}$ for $4 \mathrm{~h}$ and then poured onto saturated aq $\mathrm{NaHCO}_{3}(10 \mathrm{~mL})$, extracted with $\mathrm{Et}_{2} \mathrm{O}(2 \times 20 \mathrm{~mL})$, and the extracts dried over $\mathrm{MgSO}_{4}$. Concentration in vacuo followed by flash chromatography on silica gel with hexanes:EtOAc (20:1) gave 3.19 as a clear oil $(8 \mathrm{mg}, 10 \%)$ as one detectable isomer.

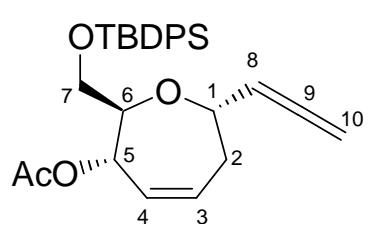

${ }^{1} \mathrm{H}$ NMR: $\delta_{\mathrm{H}}=7.69(\mathrm{~m}, 4 \mathrm{H}, \mathrm{Ar}-\mathrm{H}), 7.39(\mathrm{~m}, 6 \mathrm{H}, \mathrm{Ar}-\mathrm{H}), 5.81(\mathrm{~m}$, $1 \mathrm{H}, \mathrm{H}-3), 5.65$ (m, 1H, H-4), 5.48 (m, 1H, H-5), 5.39 (dt, J=6.5, $5.4 \mathrm{~Hz}, 1 \mathrm{H}, \mathrm{H}-8), 4.82(\mathrm{dd}, J=6.6,3.9, \mathrm{~Hz}, 2 \mathrm{H}, \mathrm{H}-10), 4.58$ (m, 1H, H-1), 4.04 (m, 1H, H-6), 3.71 (m, 2H, H-7), 2.63 (m, 1H, H-2a), 2.35 (m, 1H, H2b), 1.93 (s, 3H, H-Ac- $\left.\underline{\mathrm{CH}}_{3}\right), 1.06$ (s, 9H, H- $\left.{ }^{t} \mathrm{Bu}-\underline{\mathrm{CH}}_{3}\right)$. 
${ }^{13} \mathrm{C}$ NMR: $\delta_{\mathrm{C}}=207.8(\mathrm{C}-9), 169.8(\mathrm{CO}), 135.7(\mathrm{C}-\mathrm{Ar}), 133.4(\mathrm{C}-\mathrm{Ar}), 129.6(\mathrm{C}-\mathrm{Ar})$, 128.7 (C-4), 128.0 (C-3), 127.6 (C-Ar), 92.7 (C-8), 77.0 (C-10), 74.0 (C-6), 71.6 (C-1), 71.0 (C-5), 64.8 (C-7), $32.6(\mathrm{C}-2), 26.8\left(\mathrm{C}-{ }^{t} \mathrm{Bu}-\underline{\mathrm{CH}}_{3}\right), 26.7\left(\mathrm{C}-{ }^{t} \mathrm{Bu}-\underline{\mathrm{CH}}_{3}\right), 21.0(\mathrm{C}-\mathrm{Ac}-$ $\left.\underline{\mathrm{CH}}_{3}\right), 19.2(\mathrm{Si}-\mathrm{C})$.

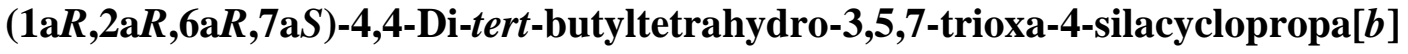

naphthalene-2-one (3.20)

To a solution of oxalyl chloride $(197 \mu \mathrm{L}, 2.28 \mathrm{mmol})$ in $\mathrm{CH}_{2} \mathrm{Cl}_{2}(8 \mathrm{~mL})$ at $-78{ }^{\circ} \mathrm{C}$ was added dropwise DMSO (324 $\mu \mathrm{L}, 4.56 \mathrm{mmol})$ and stirred 15 minutes. Glucal 1.61 (570 $\mathrm{mg}, 1.9 \mathrm{mmol})$ in $\mathrm{CH}_{2} \mathrm{Cl}_{2}(3 \mathrm{~mL})$ was added dropwise and the mixture stirred for 30 min. $\mathrm{Et}_{3} \mathrm{~N}(1.06 \mathrm{~mL}, 7.6 \mathrm{mmol})$ was then added dropwise and the mixture was stirred for $90 \mathrm{~min}$. The mixture was warmed to room temperature, poured onto saturated aq $\mathrm{NaHCO}_{3}(30 \mathrm{~mL})$ and extracted with $\mathrm{CH}_{2} \mathrm{Cl}_{2}(2 \times 30 \mathrm{~mL})$. The combined organic washings were then washed with aq $\mathrm{Na}_{2} \mathrm{SO}_{3}(2 \times 30 \mathrm{~mL})$ and saturated brine $(3 \times 20$ $\mathrm{mL})$ and concentrated in vacuo to give $\mathbf{3 . 2 0}$ as a white solid $(5.14 \mathrm{~g}, 90 \%)$.

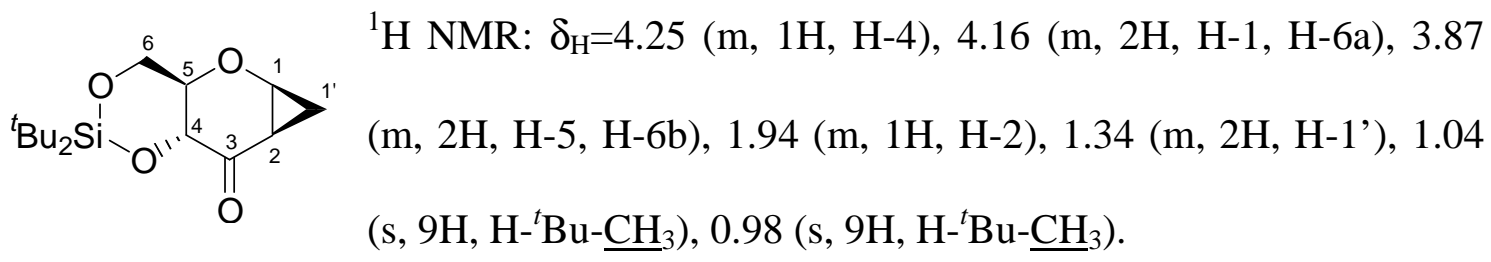

${ }^{13} \mathrm{C}$ NMR: $\delta_{\mathrm{C}}=201.6(\mathrm{C}-3), 77.9(\mathrm{C}-5), 77.7(\mathrm{C}-4), 65.6(\mathrm{C}-7), 58.7(\mathrm{C}-1), 27.3\left(\mathrm{C}-{ }^{t} \mathrm{Bu}-\right.$ $\left.\underline{\mathrm{CH}}_{3}\right), 26.8\left(\mathrm{C}-{ }^{t} \mathrm{Bu}-\underline{\mathrm{CH}}_{3}\right), 25.6(\mathrm{C}-2), 22.7$ (C-1'), 20.2 (Si-C), 20.1 (Si-C).

ES HRMS m/z: calculated for $\mathrm{C}_{15} \mathrm{H}_{26} \mathrm{O}_{4} \mathrm{Si}+\mathrm{Na} 321.1493$. Found 321.1477.

IR: $2933,2858,1711,1472,1145,1058,824,763,652 \mathrm{~cm}^{-1}$.

$\mathrm{Mp}: 137^{\circ} \mathrm{C}$.

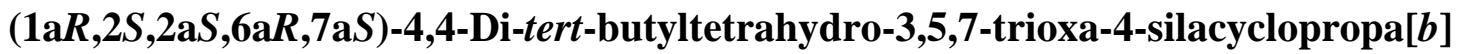
naphthalene-2-ol (3.21). 
To a mixture of $\mathbf{3 . 2 0}$ (400 mg, $1.34 \mathrm{mmol})$ in $\mathrm{EtOH}(5 \mathrm{~mL})$ was added $\mathrm{NaBH}_{4}(56 \mathrm{mg}$, $1.47 \mathrm{mmol}$ ) and the reaction stirred overnight at ambient temperature. The resulting mixture was poured onto $\mathrm{Et}_{2} \mathrm{O}(30 \mathrm{~mL})$ and washed with $\mathrm{H}_{2} \mathrm{O}(3 \times 30 \mathrm{~mL})$. The organic phase was dried over $\mathrm{MgSO}_{4}$ and concentrated in vacuo. Flash chromatography on silica gel with hexanes:EtOAc (5:1) gave the desired product $\mathbf{3 . 2 1}$ as a clear oil (95 mg, $24 \%$ ) along with starting material (200 mg, 50\%).

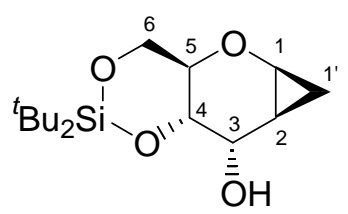

${ }^{1} \mathrm{H}$ NMR: $\delta=4.40(\mathrm{~d}, J=3.6 \mathrm{~Hz}, 1 \mathrm{H}, \mathrm{H}-3), 4.12(\mathrm{dd}, J=10.5,4.9 \mathrm{~Hz}$,

1H, H-6a), 3.73 (m, 1H, H-5), 3.67 (m, 2H, H-1, H-4), 3.64 (m,

1H, H-6b), 2.83 (s, 1H, -OH), 1.34 (dt, J=11, $7.3 \mathrm{~Hz}, 1 \mathrm{H}, \mathrm{H}-2)$,

1.03 ( s, 9H, H- $\left.{ }^{t} \mathrm{Bu}-\underline{\mathrm{CH}}_{3}\right), 1.00,\left(\mathrm{~s}, 9 \mathrm{H}, \mathrm{H}-{ }^{t} \mathrm{Bu}-\underline{\mathrm{CH}}_{3}\right), 0.74$ ( dt, J=11, $5.8 \mathrm{~Hz}, \mathrm{H}-1$ ' a), 0.39 (ddd, $J=7.6,6.4,2.4 \mathrm{~Hz}, 1 \mathrm{H}, \mathrm{H}-1$ 'b).

${ }^{13} \mathrm{C}$ NMR: $\delta_{\mathrm{C}}=74.6(\mathrm{C}-4), 66.4(\mathrm{C}-5), 66.3(\mathrm{C}-6), 65.0(\mathrm{C}-3), 51.0(\mathrm{C}-1), 27.5\left(\mathrm{C}-{ }^{t} \mathrm{Bu}-\right.$ $\left.\underline{\mathrm{CH}}_{3}\right), 27.1\left(\mathrm{C}-{ }^{t} \mathrm{Bu}-\underline{\mathrm{CH}}_{3}\right), 22.8$ (Si-C), 20.2 (Si-C), 18.0 (C-2), 10.6 (C-1').

Mp: $86^{\circ} \mathrm{C}$.

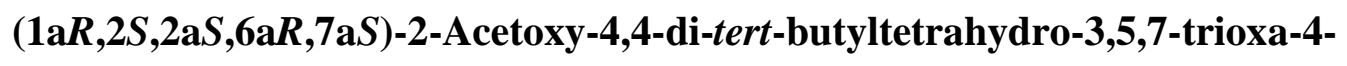
silacyclopropa[b]naphthalene (3.22).

To a mixture of $\mathbf{3 . 2 1}(95 \mathrm{mg}, 0.32 \mathrm{mmol})$ in $\mathrm{CH}_{2} \mathrm{Cl}_{2}(5 \mathrm{~mL})$ was added py $(78 \mu \mathrm{L}, 0.95$ mmol) and $\mathrm{Ac}_{2} \mathrm{O}(63 \mathrm{~mL}, 0.64 \mathrm{mmol})$ and a catalytic amount of DMAP. The reaction was stirred at ambient temperature until complete by TLC (12 h). The mixture was poured onto saturated aq $\mathrm{NaHCO}_{3}(20 \mathrm{~mL})$ and washed with $\mathrm{H}_{2} \mathrm{O}(3 \times 10 \mathrm{~mL})$. The organic phase was dried over $\mathrm{MgSO}_{4}$ and concentrated in vacuo. Column chromatography on silica gel with hexanes:EtOAc (1:1) gave 3.22 as a colourless oil (101 mg, 89\%).

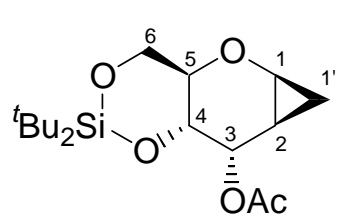

${ }^{1} \mathrm{H}$ NMR: $\delta_{\mathrm{H}}=5.61(\mathrm{~d}, J=3.5 \mathrm{~Hz}, 1 \mathrm{H}, \mathrm{H}-3), 4.08(\mathrm{dd}, J=10.5,4.9$

$\mathrm{Hz}, 1 \mathrm{H}, \mathrm{H}-6 \mathrm{a}), 3.78$ (dd, $J=9.9,4.8 \mathrm{~Hz}, 1 \mathrm{H}, \mathrm{H}-5), 3.71$ (dd, $J=$ 
9.7, $5.7 \mathrm{~Hz}, 1 \mathrm{H}, \mathrm{H}-4), 3.63$ (m, 1H, H-1), 3.61 (m, 1H, H-6b), 2.11 (s, 3H, H-Ac- $\underline{\mathrm{CH}}_{3}$ ), 1.21 (m, 1H, H-2), 0.99 (s, 9H, H- $\left.{ }^{t}{ }^{\mathrm{Bu}}-\underline{\mathrm{CH}}_{3}\right), 0.97$ (s, 9H, H- $\left.{ }^{t} \mathrm{Bu}-\underline{\mathrm{CH}}_{3}\right), 0.75$ (m, 1H, H1'a), 0.47 (ddd, $J=7.4,6.6,2.5 \mathrm{~Hz}, 1 \mathrm{H}, \mathrm{H}-1$ 'b).

${ }^{13} \mathrm{C}$ NMR: $\delta_{\mathrm{C}}=170.6(\mathrm{C}-\mathrm{Ac}), 72.8(\mathrm{C}-4), 67.9(\mathrm{C}-3), 67.4(\mathrm{C}-5), 66.2(\mathrm{C}-6), 50.6(\mathrm{C}-1)$, $27.4\left(\mathrm{C}-{ }^{t} \mathrm{Bu}-\underline{\mathrm{CH}}_{3}\right), 26.8\left(\mathrm{C}-{ }^{t} \mathrm{Bu}-\underline{\mathrm{CH}}_{3}\right) 22.7$ (C-Ac), 21.5 (Si-C), 20.1 (Si-C), 17.4 (C-2), $12.0\left(\mathrm{H}-1^{\prime}\right)$.

ES HRMS m/z: calculated for $\mathrm{C}_{15} \mathrm{H}_{27} \mathrm{O}_{3} \mathrm{Si}+\mathrm{H}$ (parent ion - OAc group) 284.18076. Found 284.0307.

\section{7-(Ethyl-2'-ethanoate)-2,7-dihydro-oxepin-2-methoxy-tert-butyldimethylsilane}

To a solution of glucal $3.16(87 \mathrm{mg}, 0.18 \mathrm{mmol})$ in acetonitrile $(1 \mathrm{~mL})$ at $0{ }^{\circ} \mathrm{C}$ was added $3.9(300 \mu \mathrm{L}, 1.44 \mathrm{mmol})$ and TMSOTf $(15 \mu \mathrm{L}, 0.09 \mathrm{mmol})$. The reaction warmed to ambient temperature and stirred for $5 \mathrm{~h}$. The mixture was poured onto saturated aq $\mathrm{NaHCO}_{3}(10 \mathrm{~mL})$, extracted with $\mathrm{Et}_{2} \mathrm{O}(2 \times 20 \mathrm{~mL})$, and the extracts dried over $\mathrm{MgSO}_{4}$. Concentration in vacuo followed by flash chromatography on silica gel with hexanes:EtOAc (50:1) gave 4.1 as a clear oil $(46 \mathrm{mg}, 57 \%)$ as a $6: 1$ mixture of products by GC.

Major isomer: (2S,7R)-7-(Ethyl-2'-ethanoate)-2,7-dihydro-oxepin-2-methoxy-tertbutyldiphenylsilane (4.1a).

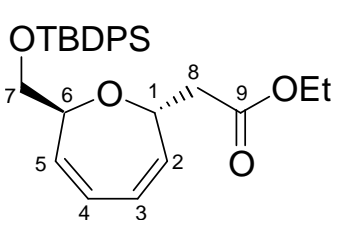

${ }^{1} \mathrm{H}$ NMR: $\delta_{\mathrm{H}}=7.65(\mathrm{~m}, 4 \mathrm{H}, \mathrm{H}-\mathrm{Ar}), 7.39(\mathrm{~m}, 6 \mathrm{H}, \mathrm{H}-\mathrm{Ar}), 6.16(\mathrm{~m}$, 1H, H-5), 6.05 (m, 1H, H-4), 6.04 (m, 1H, H-3), 5.98 (m, 1H, H2), 4.78 (m, 1H, H-1), 4.26 (m, 1H, H-6), 4.05 (q, J=7 Hz, 2H, O$\left.\underline{\mathrm{CH}}_{2}-\mathrm{CH}_{3}\right), 3.75$ (dd, J=6.1, 4.4 Hz, 2H, H-7), 2.63 (t, J=6.6 Hz, 2H, H-8), 1.17 (t, $\left.J=6.1 \mathrm{~Hz}, 3 \mathrm{H}, \mathrm{O}-\mathrm{CH}_{2}-\underline{\mathrm{CH}}_{3}\right), 1.05\left(\mathrm{~s}, 9 \mathrm{H}, \mathrm{H}-{ }^{t} \mathrm{Bu}-\underline{\mathrm{CH}}_{3}\right)$. 
${ }^{13} \mathrm{C}$ NMR: $\delta_{\mathrm{C}}=170.7(\mathrm{C}-9), 137.0(\mathrm{C}-2), 136.2(\mathrm{C}-5), 135.6(\mathrm{C}-\mathrm{Ar}), 133.4(\mathrm{C}-\mathrm{Ar}), 129.7$ (C-Ar), 127.7 (C-Ar), 126.8 (C-3), 126.7 (C-4), 75.4 (C-6), 72.7 (C-1), 65.7 (C-7), 60.5 $\left(\mathrm{O}-\underline{\mathrm{CH}}_{2}-\mathrm{CH}_{3}\right), 40.9(\mathrm{C}-8), 26.8\left(\mathrm{C}-{ }^{t} \mathrm{Bu}-\underline{\mathrm{CH}}_{3}\right), 19.2(\mathrm{Si}-\mathrm{C}), 14.1\left(\mathrm{O}-\mathrm{CH}_{2}-\underline{\mathrm{CH}}_{3}\right)$.

ES HRMS $m / z$ : calculated for $\mathrm{C}_{27} \mathrm{H}_{34} \mathrm{O}_{4} \mathrm{Si}+\mathrm{NH}_{4}$ 468.2565. Found 468.2556. IR: 2931, 2858, 1734, 1427, 1109, 910, 823, 734, $701 \mathrm{~cm}^{-1}$.

Minor isomer: (2S,7S)-7-(Ethyl-2'-ethanoate)-2,7-dihydro-oxepin-2-methoxy-tertbutyldiphenylsilane $(4.1 \mathrm{~b})$.

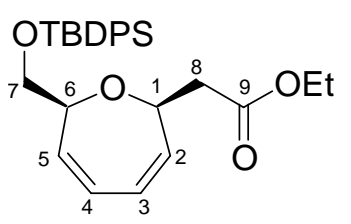

${ }^{1} \mathrm{H}$ NMR: $\delta_{\mathrm{H}}=7.65(\mathrm{~m}, 4 \mathrm{H}, \mathrm{H}-\mathrm{Ar}), 7.39(\mathrm{~m}, 6 \mathrm{H}, \mathrm{H}-\mathrm{Ar}), 6.02(\mathrm{~m}$, 1H, H-5), 5.91 (m, 1H, H-4), 5.87 (m, 1H, H-3), 5.82 (m, 1H, H2), $4.83(\mathrm{~m}, 1 \mathrm{H}, \mathrm{H}-1), 4.56$ (m, 1H, H-6), 4.10 (q, J=7.1 Hz, 2H, O- $\underline{\mathrm{CH}}_{2}-\mathrm{CH}_{3}$ ), 3.81 ( dd, J=6.4, $9.8 \mathrm{~Hz}, 1 \mathrm{H}, \mathrm{H}-7$ ), 3.60 (dd, J=6.9, $10.0 \mathrm{~Hz}, 1 \mathrm{H}, \mathrm{H}-7$ ), $2.70(\mathrm{dd}, J=8.8,15.6 \mathrm{~Hz}, 1 \mathrm{H}, \mathrm{H}-8), 2.51$ (dd, $J=5.6,15.6 \mathrm{~Hz}, 1 \mathrm{H}, \mathrm{H}-8), 1.17$ (t, $J=6.1$ $\left.\mathrm{Hz}, 3 \mathrm{H}, \mathrm{O}-\mathrm{CH}_{2}-\underline{\mathrm{CH}}_{3}\right), 1.05$ (s, 9H, H- $\left.{ }^{t} \mathrm{Bu}-\underline{\mathrm{CH}}_{3}\right)$.

${ }^{13} \mathrm{C}$ NMR: $\delta_{\mathrm{C}}=170.9(\mathrm{C}-9), 136.7$ (C-Ar), 135.61 (C-2), 135.59 (C-5), 133.5 (C-Ar), 129.6 (C-Ar), 127.6 (C-Ar), 126.0 (C-3), 125.3 (C-4), 80.9 (C-6), 76.0 (C-1), 60.5 (O$\left.\underline{\mathrm{CH}_{2}}-\mathrm{CH}_{3}\right), 40.3(\mathrm{C}-8), 26.7\left(\mathrm{C}-{ }^{t} \mathrm{Bu}-\underline{\mathrm{CH}} 3\right), 19.2(\mathrm{Si}-\mathrm{C}), 14.1\left(\mathrm{O}-\underline{\mathrm{CH}_{2}}-\mathrm{CH}_{3}\right)$.

ES HRMS $m / z$ : calculated for $\mathrm{C}_{27} \mathrm{H}_{34} \mathrm{O}_{4} \mathrm{Si}+\mathrm{NH}_{4}$ 486.2565. Found 468.2556. IR: 2931, 2858, 1734, 1427, 1110, 1028, 909, 823, 733, $701 \mathrm{~cm}^{-1}$.

\section{(2S,7R)-7-(Ethyl-1',1'-dimethyl-2'-ethanoate)-2,7-dihydro-oxepin-2-methoxy-tert-}

\section{butyldiphenylsilane (4.2)}

To a solution of cyclopropanated glucal 3.16 (90 mg, $0.187 \mathrm{mmol})$ in $\mathrm{MeCN}(1 \mathrm{~mL})$ at $0{ }^{\circ} \mathrm{C}$ was added $3.10(195 \mu \mathrm{L}, 0.96 \mathrm{mmol})$ and TMSOTf $(16 \mu \mathrm{L}, 0.094 \mathrm{mmol})$. The reaction was stirred overnight warming to ambient temperature and then poured onto saturated aq $\mathrm{NaHCO}_{3}(10 \mathrm{~mL})$, extracted with $\mathrm{Et}_{2} \mathrm{O}(2 \times 20 \mathrm{~mL})$, and the extracts dried 
over $\mathrm{MgSO}_{4}$. Concentration in vacuo followed by flash chromatography on silica gel with hexanes:EtOAc (50:1) gave $\mathbf{4 . 2}$ as a clear oil (13 mg, 10\%) as one isomer.

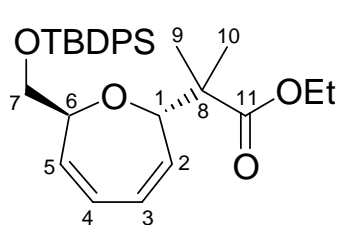

${ }^{1} \mathrm{H}$ NMR: $\delta_{\mathrm{H}}=7.67(\mathrm{~m}, 4 \mathrm{H}, \mathrm{H}-\mathrm{Ar}), 7.42(\mathrm{~m}, 6 \mathrm{H}, \mathrm{H}-\mathrm{Ar}), 6.13(\mathrm{~m}$, 1H, H-5), 6.07 (s, 2H, H-3, H-4), 6.05 (m, 1H, H-2), 4.42 (t, J=5.9 $\mathrm{Hz}, 1 \mathrm{H}, \mathrm{H}-6), 4.33$ (dd, $J=4.2,2.0 \mathrm{~Hz}, 1 \mathrm{H}, \mathrm{H}-1$ ), 4.06 (dq, $J=11.0$, 7.1Hz, $\left.1 \mathrm{H}, \mathrm{O}-\underline{\mathrm{CH}_{2}}-\mathrm{CH}_{3}\right), 3.93\left(\mathrm{dq}, J=10.7,7.1 \mathrm{~Hz}, 1 \mathrm{H}, \mathrm{O}-\underline{\mathrm{CH}}_{2}-\mathrm{CH}_{3}\right), 3.73(\mathrm{dd}, J=6.1$, $0.9 \mathrm{~Hz}, 2 \mathrm{H}, \mathrm{C}-7), 1.27$ (s, 3H, H-9), 1.20 (s, 3H, H-10), 1.13 (t, J=7.1 Hz, 3H, H-Ac), 1.06 (s, 9H, H- $\left.{ }^{t} \mathrm{Bu}-\underline{\mathrm{CH}}_{3}\right)$.

${ }^{13} \mathrm{C}$ NMR: $\delta_{\mathrm{C}}=176.5(\mathrm{C}-11), 136.3(\mathrm{C}-4), 135.6(\mathrm{C}-\mathrm{Ar}), 135.6(\mathrm{C}-\mathrm{Ar}), 134.9(\mathrm{C}-2)$, 129.7 (C-Ar), 129.7 (C-Ar), 128.8 (C-5), 127.7 (C-Ar), 126.3 (C-3), 78.8 (C-6), 77.0 (C-1), 66.3 (C-7), $60.4\left(\mathrm{O}-\mathrm{CH}_{2}-\underline{\mathrm{CH}}_{3}\right), 46.3$ (C-8), 26.8 (C- $\left.{ }^{t} \mathrm{Bu}-\underline{\mathrm{CH}}_{3}\right), 21.9$ (C-9), 20.5 (C-10), 19.2 (Si-C), $14.1\left(\mathrm{O}-\mathrm{CH}_{2}-\underline{\mathrm{CH}}_{3}\right)$.

\section{2,7-Diallyl-2,3,6,7-tetrahydro-oxepin-2-methoxy-tert-butyldiphenylsilane (4.3)}

a) To a solution of cyclopropane $3.16(102 \mathrm{mg}, 0.212 \mathrm{mmol})$ in $\mathrm{MeCN}(1 \mathrm{~mL})$ at $-40{ }^{\circ} \mathrm{C}$ was added TMSallyl $(157 \mu \mathrm{L}, 1.06 \mathrm{mmol})$ and TMSOTf $(11.5 \mu \mathrm{L}, 0.64 \mathrm{mmol})$. The reaction was stirred for $4 \mathrm{~h}$ while warming to room temperature. Excess solid $\mathrm{NaHCO}_{3}$ was added to the mixture and stirred for $5 \mathrm{~min}$. The mixture was then poured onto a silica plug, eluted with hexanes $(100 \mathrm{~mL})$ and concentrated in vacuo to give $\mathbf{4 . 3}$ as a clear oil (51mg, 54\%).

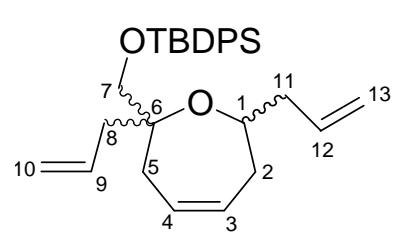

${ }^{1} \mathrm{H}$ NMR: $\delta_{\mathrm{H}}=7.69(\mathrm{~m}, 4 \mathrm{H}, \mathrm{Ar}-\mathrm{H}), 7.39(\mathrm{~m}, 6 \mathrm{H}, \mathrm{Ar}-\mathrm{H}), 5.92$ (m, 1H, H-9), 5.82 (m, 1H, H-12), 5.51 (m, 1H, H-4), 5.43 (m, 1H, H-3), 5.12 (m, 2H, H-10), 5.03 (m, 2H, H13), 4.12 (m, 1H, H1), 3.56 (d, J=9.5 Hz, H-7), 3.47 (d, $J=9.5 \mathrm{~Hz}, \mathrm{H}-7), 2.55$ (m, 1H, H-2), 2.45 (m, 1H, H-8), 2.41 (m, 1H, H-8), 2.30 (m, 1H, H-2), 2.25 (m, 1H, H-5), 2.23 (m, 1H, H-11), $2.10(\mathrm{~m}, 1 \mathrm{H}, \mathrm{H}-11), 2.05$ (m, 1H, H-5), 1.05 (s, 9H, H- $\left.{ }^{t} \mathrm{Bu}^{-\mathrm{CH}_{3}}\right)$. 
${ }^{13} \mathrm{C}$ NMR: $\delta_{\mathrm{C}}=135.7(\mathrm{C}-12), 135.6(\mathrm{C}-9), 134.5(\mathrm{C}-\mathrm{Ar}), 134.4(\mathrm{C}-\mathrm{Ar}), 134.3(\mathrm{C}-\mathrm{Ar})$, 133.6 (C-Ar), 133.5 (C-Ar), 130.2 (C-4), 129.6 (C-Ar), 129.5 (C-Ar), 127.9 (C-Ar), 127.6 (C-Ar), 127.5 (C-Ar), 124.5 (C-3), 117.4 (C-10), 116.6 (C-13), 81.5 (C-6), 69.9 (C-1), 66.2 (C-7), 41.7 (C-11), 40.4 (C-8), 38.7 (C-5), 32.3 (C-2), 26.9 (C- $\left.{ }^{t} \mathrm{Bu}^{-\mathrm{CH}_{3}}\right)$, 19.3 (Si-C).

b) To a solution of cyclopropane $\mathbf{3 . 1 6}(100 \mathrm{mg}, 0.207 \mathrm{mmol})$ in $\mathrm{MeCN}(1 \mathrm{~mL})$ at $-40{ }^{\circ} \mathrm{C}$ was added TMSallyl $(40 \mu \mathrm{L}, 0.248 \mathrm{mmol})$ and TMSOTf $(11 \mu \mathrm{L}, 0.62 \mathrm{mmol})$. The reaction was stirred for $1 \mathrm{~h}$ while warming to room temperature then poured onto saturates aq $\mathrm{NaHCO}_{3}(10 \mathrm{~mL})$, extracted with $\mathrm{Et}_{2} \mathrm{O}(2 \times 20 \mathrm{~mL})$, and the combined extracts dried over $\mathrm{MgSO}_{4}$. Concentration in vacuo followed by flash chromatography on silica gel with hexanes:EtOAc (25:1) gave 3 compounds with some recovered starting material (23 mg, 23\%)

\section{(2S,7R)-7-Allyl-2,7-dihydro-oxepin-2-methoxy-tert-butyldiphenylsilane (4.4)}

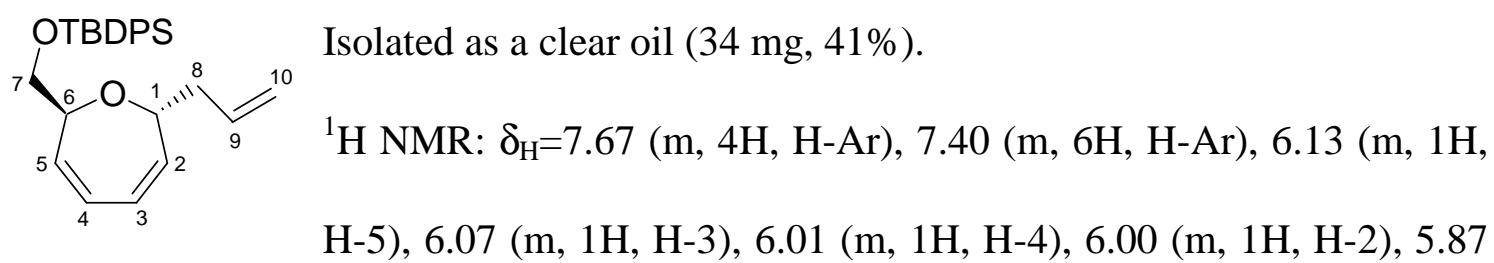
(m, 1H, H-9), 5.07 (m, 1H, H-10), 5.03 (m, 1H, H-10), 4.39 (td, J=7.3, $2.7 \mathrm{~Hz}, 1 \mathrm{H}, \mathrm{H}-$ 1), 4.35 (m, 1H, H-6), 3.80 (dd, $J=10.5,6.4 \mathrm{~Hz}, 1 \mathrm{H}, \mathrm{H}-7 \mathrm{a}), 3.72$ (dd, $J=10.4,6.1 \mathrm{~Hz}$, 1H, H-7b), 2.40 (m, 2H, H-8), 1.08 (s, 9H, H- $\left.{ }^{t} \mathrm{Bu}-\underline{\mathrm{CH}}_{3}\right)$.

${ }^{13} \mathrm{C}$ NMR: $\delta_{\mathrm{C}}=138.1(\mathrm{C}-2), 136.1(\mathrm{C}-5), 136.0(\mathrm{C}-\mathrm{Ar}), 135.6(\mathrm{C}-9), 134.6(\mathrm{C}-\mathrm{Ar}), 133.4$ (C-Ar), 129.7 (C-Ar), 129.6 (C-Ar), 127.7 (C-Ar), 126.8 (C-3), 126 9C-4), 117 (C-10), 75.3 9C-6), 75.2 (C-1), 65.9 (C-7), 40.0 (C-8), $26.7\left(\mathrm{C}-{ }^{t} \mathrm{Bu}^{-\mathrm{CH}_{3}}\right), 19.2$ (Si-C). 


\section{$(2 R, 3 S, 7 R)$-7-Allyl-3-acetate-2-(tert-butyldiphenylsilanyloxymethyl)-2,3,6,7-}

\section{tetrahydro-oxepin-4-ene (4.5)}

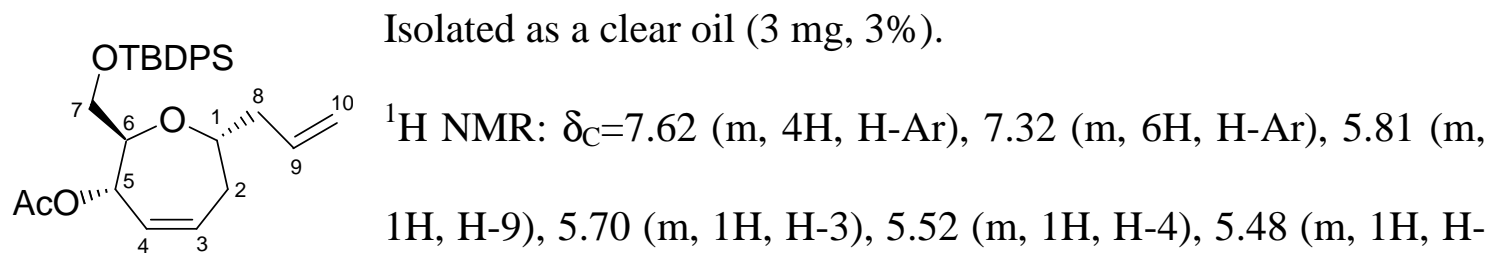

5), 5.00 (m, 1H, H-10a), 4.96 (m, 1H, H-10b), 3.99 (m, 1H, H-1), 3.95 (m, 1H, H-6), 3.64 (d, J= 3.9 Hz, 1H, H-7), 2.50 (m, 1H, H-8a), 2.40 (m, 1H, H-2a), 2.30 (m, 1H, H2b), 2.20 (m, 1H, H-8b), 1.84 (s, 3H, H-Ac- $\underline{\mathrm{CH}}_{3}$ ), 0.99 (s, 9H, H- $\left.{ }^{t} \mathrm{Bu}-\underline{\mathrm{CH}}_{3}\right)$.

\section{$(1 R, 2 S, 6 R)$-2-Acetate-7,9-dioxa-bicyclo[4.2.1]non-3-ene (4.6)}

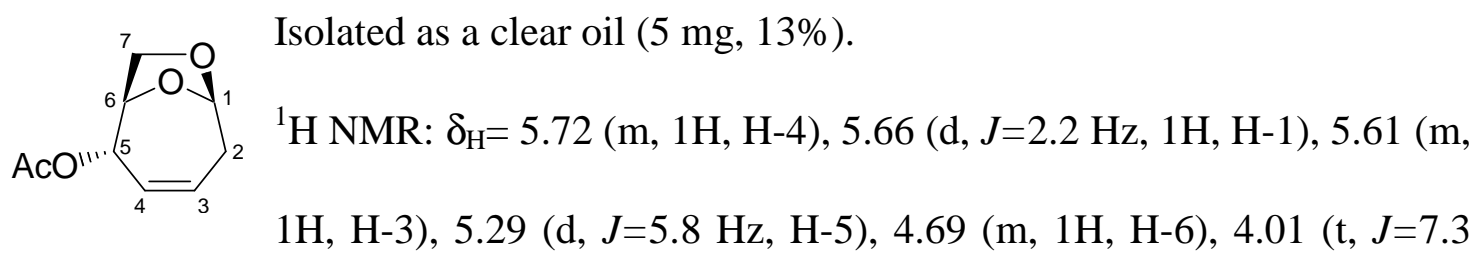

Hz, 1H, H-7a), 3.88 (dd, J=7.8, 2.2 Hz, 1H, H-7b), 2.62 (dd, J=10.4, 2.2 Hz, 1H, H-2a), 2.58 (dd, $J=10.8,5.8 \mathrm{~Hz}, 1 \mathrm{H}, \mathrm{H}-2 \mathrm{~b}$ ), 2.10 (s, 3H, H-Ac- $\left.\underline{\mathrm{CH}}_{3}\right)$.

${ }^{13} \mathrm{C}$ NMR: $\delta_{\mathrm{C}}=131(\mathrm{C}-4), 12(\mathrm{C}-3), 104$ (C-1), 78 (C-6), 75 ((C-5), 68 (C-7), 39 (C-2), $21(\mathrm{C}-\mathrm{Ac}-\underline{\mathrm{CH}} 3)$.

\section{2(S)-2-O-(Di-tert-butyl-O-triethylsilyl)silyl-2-hydroxymethyl-2,3,6,7-tetrahydro- oxepin-4-ene(4.7).}

To a solution of $3.4(155 \mathrm{mg}, 0.45 \mathrm{mmol})$ in $\mathrm{MeCN}(1.0 \mathrm{~mL})$ at $0{ }^{\circ} \mathrm{C}$ was added $\mathrm{Et}_{3} \mathrm{SiH}$ ( $720 \mu \mathrm{L}, 4.5 \mathrm{mmol})$ then TMSOTf $(20 \mu \mathrm{L}, 0.113 \mathrm{mmol})$. The solution was allowed to warm to ambient temperature and stirred $4 \mathrm{~h}$ then poured onto saturates aq $\mathrm{NaHCO}_{3}(10$ $\mathrm{mL})$, extracted with $\mathrm{Et}_{2} \mathrm{O}(3 \times 20 \mathrm{~mL})$ and dried over $\mathrm{MgSO}_{4}$. Concentration in vacuo followed by flash chromatography on silica gel with hexanes:EtOAc (100:1) gave 4.7 as a colourless oil $(111 \mathrm{mg}, 86 \%)$. 


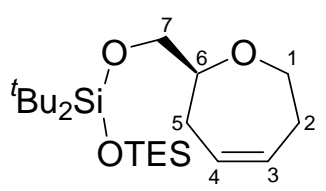

${ }^{1} \mathrm{H}$ NMR: $\delta_{\mathrm{H}}=5.84(\mathrm{~d}, J=3.4 \mathrm{~Hz}, 1 \mathrm{H}, \mathrm{H}-4), 5.83(\mathrm{~d}, J=3.9 \mathrm{~Hz}, 1 \mathrm{H}$, H-3), 4.06 (dt, $J=11.9,3.9 \mathrm{~Hz}, 1 \mathrm{H}, \mathrm{H}-7), 3.86$ (dd, $J=9.5,4.9 \mathrm{~Hz}$,

1H, H-6), 3.56 (dt, J=9.5, 7.6 Hz, 1H, H-6), 3.46 (m, 2H, H-2, H-8), 2.50 (bm, 2H, H-2, H-5), 2.20 (bm, 2H, H-2, H-5), 0.9 (m, 27H, H- $\left.{ }^{t} \mathrm{Bu}^{-\mathrm{CH}_{3}}, \mathrm{H}-\mathrm{CH}_{2}-\mathrm{CH}_{3}\right), 0.66$ (q, $\left.J=8.1 \mathrm{~Hz}, 6 \mathrm{H}, \mathrm{Si}-\underline{\mathrm{CH}}_{2}-\mathrm{CH}_{3}\right)$.

${ }^{13} \mathrm{CNMR}: \delta_{\mathrm{C}}=130.7$ (C-4), 129.5 (C-3), 80.7 (C-6), 69.6 (C-1), 66.4 (C-7), 33.7 (C-2),

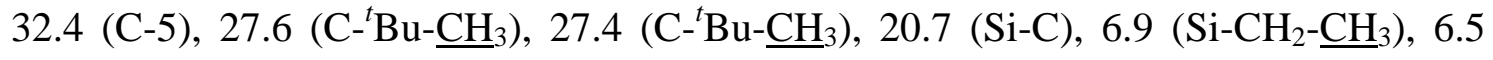
$\left(\mathrm{Si}-\underline{\mathrm{CH}}_{2}-\mathrm{CH}_{3}\right)$.

IR (neat): 2934, 2859, 1472, 1068, 826, $731 \mathrm{~cm}^{-1}$.

ES HRMS $m / z$ : calculated for $\mathrm{C}_{21} \mathrm{H}_{44} \mathrm{O}_{3} \mathrm{Si}_{2}+\mathrm{H}$ : 401.2902. Found 401.2892.

\section{Deuterium Labelling Experiment.}

To a solution of oxepane $3.4(100 \mathrm{mg}, 0.29 \mathrm{mmol})$ in $\mathrm{MeCN}(1 \mathrm{~mL})$ at $0{ }^{\circ} \mathrm{C}$ was added TESD $(226 \mu \mathrm{L}, 1.46 \mathrm{mmol})$ and TMSOTf $(26 \mu \mathrm{L}, 0.146 \mathrm{mmol})$. The reaction mixture was allowed to warm to ambient temperature and stirred until TLC revealed no starting material was left. The reaction mixture was poured onto saturates aq $\mathrm{NH}_{4} \mathrm{Cl}(10 \mathrm{~mL})$, and extracted with $\mathrm{Et}_{2} \mathrm{O}(2 \times 10 \mathrm{~mL})$. The combined organic extracts were dried over $\mathrm{MgSO}_{4}$ and concentrated in vacuo to give a colourless oil $(99 \mathrm{mg})$. Repeated flash chromatography on silica gel with hexanes to remove TES impurities gave a colourless oil (10 mg) containing two compounds. As the ${ }^{1} \mathrm{H}$ and ${ }^{13} \mathrm{C}$ NMR of these compounds were congested in the area where the protecting groups' signals appear, only the oxepine rings were definitively assigned. 
Major component: 2,7-Deutero-2-O-(di-tert-butyl-O-triethylsilyl)silyl-2-

hydroxymethyl-2,3,6,7-tetrahydro-oxepin-4-ene (4.8) as a 1:1 mixture of epimers.

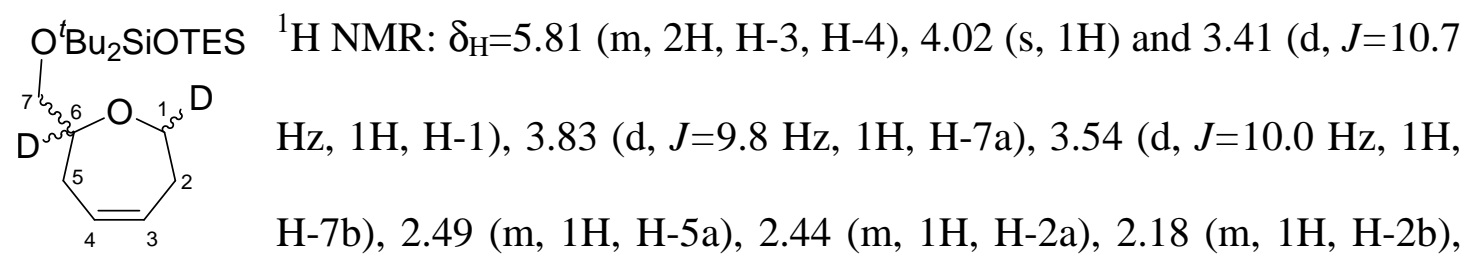

$2.16(\mathrm{~m}, 1 \mathrm{H}, \mathrm{H}-5 \mathrm{~b})$.

${ }^{13} \mathrm{C}$ NMR: $\delta_{\mathrm{C}}=130.7(\mathrm{C}-3), 129.4(\mathrm{C}-4), 80.2(\mathrm{C}-6, \mathrm{t}, J=20.5 \mathrm{~Hz}), 69.3(\mathrm{C}-1, \mathrm{t}, J=21.9$ Hz), 69.2 (C-1, t, J=21.4 Hz), 66.3 (C-7), 33.6 (C-5), 32.3 (C-2).

Minor component: 7-deutero-2- $\boldsymbol{O}$-(di-tert-butylsilyl- $\boldsymbol{O}$-triethylsilyl)-2-hydroxy methyl-2,7-dihydro-oxepin-4-ene (4.9).

$\mathrm{O}^{t} \mathrm{Bu}_{2} \mathrm{SiOTES}{ }^{1} \mathrm{H}$ NMR: $\delta_{\mathrm{H}}=6.01(\mathrm{~m}, 1 \mathrm{H}, \mathrm{H}-5), 5.98(\mathrm{~m}, 1 \mathrm{H}, \mathrm{H}-3), 5.94(\mathrm{~m}, 1 \mathrm{H}, \mathrm{H}-$<smiles>[2H][C@H]1C=CC=C[C@@H](C)O1</smiles>
4), 5.93 (m, 1H, H-2), 4.43 (d, J=2.2 Hz, 1H, H-1), 4.25 (s, 1H, H-6), 3.97 (dd, $J=10.0,6.6 \mathrm{~Hz}, 1 \mathrm{H}, \mathrm{H}-7), 3.77$ (m, 1H, H-7).

${ }^{13}$ C NMR: $\delta_{C}=135.4(\mathrm{C}-3), 135.3(\mathrm{C}-2), 126.2(\mathrm{C}-4), 125.6(\mathrm{C}-5), 80.4(\mathrm{C}-6), 70.2(\mathrm{C}-$ $1, \mathrm{t}, J=21.6 \mathrm{~Hz}), 65.1(\mathrm{C}-7)$.

\section{2-((4aR,6S,8,9,9aS)-8,9-Dibromo-2,2-di-tert-butylhexahydro-1,3,5-trioxa-2-sila benzocyclohepten-6-yl)-2-methylpropionic acid ethyl ester.}

To a solution of oxepine $\mathbf{3 . 1 2}(101 \mathrm{mg}, 0.25 \mathrm{mmol})$ in $\mathrm{CHCl}_{3}(1 \mathrm{~mL})$ at ambient temperature was added bromine $(19 \mu \mathrm{L}, 0.38 \mathrm{mmol}, 1.5 \mathrm{eq})$ and stirred for $30 \mathrm{~min}$. The mixture was poured onto $\mathrm{Et}_{2} \mathrm{O}(20 \mathrm{~mL})$ and washed with $\mathrm{H}_{2} \mathrm{O}(2 \times 20 \mathrm{~mL})$ and brine $(1$ x $20 \mathrm{~mL}$ ). The organic layer was dried over $\mathrm{MgSO}_{4}$ and the solvent removed in vacuo. Flash chromatography on silica with hexanes:EtOAc (9:1) gave $\mathbf{5 . 1}$ and $\mathbf{5 . 2}$ as a 2:1 mix of epimers as a clear oil in $90 \%$ overall yield. 
Major fraction (85 mg, 60\%) (5.1) -8S,9S-: ${ }^{1} \mathrm{H}$ NMR:

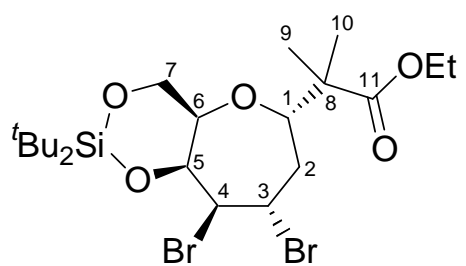

$\delta_{\mathrm{H}}=4.64(\mathrm{~d}, J=3.2 \mathrm{~Hz}, 1 \mathrm{H}, \mathrm{H}-5), 4.45(\mathrm{td}, J=11.0,1.7 \mathrm{~Hz}$,

1H, H-3), 4.23-4.0 (m, 6H, H-1, H-4, H-7, O- $\left.\underline{\mathrm{CH}}_{2}-\mathrm{CH}_{3}\right)$, 3.85 (s, 1H, H-6), 2.57 (dt, J=15.4, $2.2 \mathrm{~Hz}, 1 \mathrm{H}, \mathrm{H}-2 \mathrm{a}), 2.35$ (dt, J=15.4, $11.5 \mathrm{~Hz}, 1 \mathrm{H}$, H-2b), 1.27 (t, J=7.1 Hz, 3H, O- $\mathrm{CH}_{2}-\underline{\mathrm{CH}_{3}}$ ), 1.19 (s, 3H, H-9), 1.15 (s, 3H, H-10), 1.08 (s, $\left.18 \mathrm{H}, \mathrm{H}^{-}{ }^{t} \mathrm{Bu}-\underline{\mathrm{CH}}_{3}\right)$.

${ }^{13} \mathrm{C}$ NMR: $\delta_{\mathrm{C}}=175.8(\mathrm{C}-11), 79.2(\mathrm{C}-1), 75.9(\mathrm{C}-5), 71.9$ (C-6), $70.4(\mathrm{C}-7), 62.8(\mathrm{C}-4)$, $60.7\left(\mathrm{O}-\underline{\mathrm{CH}}_{2}-\mathrm{CH}_{3}\right), 52.9(\mathrm{C}-3), 47.9(\mathrm{C}-8), 38.6(\mathrm{C}-2), 27.6\left(\mathrm{C}-{ }^{t} \mathrm{Bu}-\underline{\mathrm{CH}}_{3}\right), 23.5(\mathrm{C}-9)$, 20.9 (C-10), 20.6 ( $\mathrm{Si}-\mathrm{C}), 14.1\left(\mathrm{O}-\mathrm{CH}_{2}-\underline{\mathrm{CH}_{3}}\right)$.

ES HRMS $m / z$ : calculated for $\mathrm{C}_{21} \mathrm{H}_{38} \mathrm{O}_{5} \mathrm{BR}_{2} \mathrm{Si}+\mathrm{H}$ 557.0928. Found 557.0945.

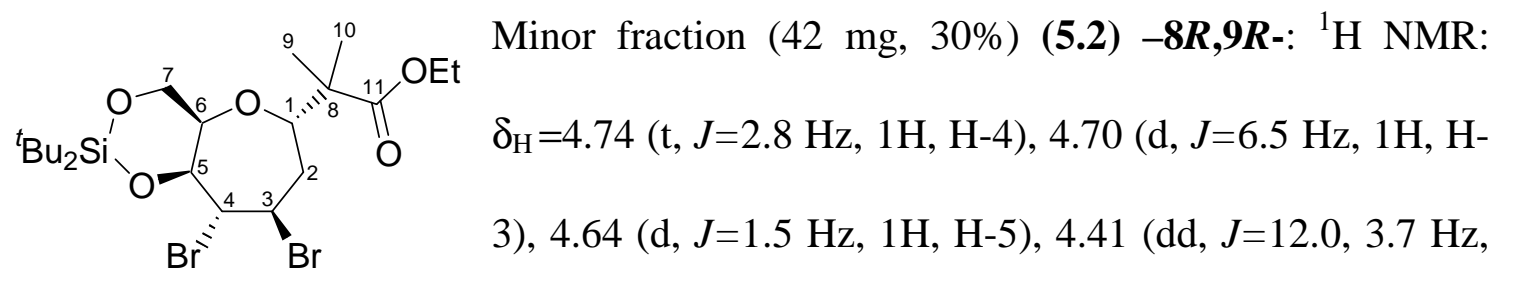
1H, H-1), 4.22 (dd, J=11.4, 1.9 Hz, 1H, H-7a), 4.18 (dd, J=10.7, 7.0 Hz, 1H, O- $\underline{\mathrm{CH}}_{2}-$ $\left.\mathrm{CH}_{3}\right), 4.12$ (dd, J=11.4, 1.9 Hz, 1H, H-7b), 4.07 (m, 1H, H-6), 4.06 (dd, J=10.7, 7.0 Hz, $\left.1 \mathrm{H}, \mathrm{O}-\underline{\mathrm{CH}}_{2}-\mathrm{CH}_{3}\right), 2.80(\mathrm{ddd}, 1 \mathrm{H}, J=15.6,12.2,1.2 \mathrm{~Hz}, \mathrm{H}-2 \mathrm{a}), 1.97$ (dt, 1H, $J=16.6$, $1.2 \mathrm{~Hz}, \mathrm{H}-2 \mathrm{~b}$ ), 1.30 (t, 3H, J=7.1 Hz, O- $\mathrm{CH}_{2}-\underline{\mathrm{CH}}_{3}$ ), 1.25 (s, 3H, H-9), 1.24 (s, 3H, H10), 1.09 (s, 9H, H- $\left.{ }^{t} \mathrm{Bu}-\underline{\mathrm{CH}}_{3}\right), \mathrm{d} 1.04\left(\mathrm{~s}, 9 \mathrm{H}, \mathrm{H}-{ }^{t} \mathrm{Bu}-\underline{\mathrm{CH}}_{3}\right)$.

${ }^{13} \mathrm{C}$ NMR: $\quad \delta_{\mathrm{C}}=176(\mathrm{C}-11), 79.8(\mathrm{C}-5), 78.9(\mathrm{C}-1), 70.4(\mathrm{C}-7), 66.9(\mathrm{C}-6), 60.7(\mathrm{O}-$ $\left.\underline{\mathrm{CH}}_{2}-\mathrm{CH}_{3}\right), 57.6(\mathrm{C}-4), 48.6(\mathrm{C}-3), 47.5(\mathrm{C}-8), 28.0(\mathrm{C}-2), 27.6\left(\mathrm{C}-{ }^{t} \mathrm{Bu}-\underline{\mathrm{CH}}_{3}\right), 27.3(\mathrm{C}-$ $\left.{ }^{t} \mathrm{Bu}-\underline{\mathrm{CH}}_{3}\right), 22.5$ (C-10), 22.3 (C-9), 20.9 (Si-C), $14.1\left(\mathrm{O}-\mathrm{CH}_{2}-\underline{\mathrm{CH}}_{3}\right)$.

ES HRMS $m / z$ : calculated for $\mathrm{C}_{21} \mathrm{H}_{38} \mathrm{O}_{5} \mathrm{BR}_{2} \mathrm{Si}+\left(\mathrm{NH}_{4} \mathrm{Na}\right)$ 597.1091. Found 597.0825. 


\section{2-((2S,4S,5S,6S,7R)-4,5-Dibromo-6-hydroxy-7-hydroxymethyloxepan-2-yl)-2-}

\section{methylpropionic acid ethyl ester (5.3).}

To a solution of dibromide $5.1(32 \mathrm{mg}, 0.057 \mathrm{mmol})$ in THF (1 mL) at ambient temperature was added TBAF ( $1 \mathrm{M}$ in hexanes, $171 \mu \mathrm{L}, 0.17 \mathrm{mmol}, 3$ eq) and the reaction stirred for $2 \mathrm{~h}$. The resulting mixture was poured onto $\mathrm{Et}_{2} \mathrm{O}(20 \mathrm{~mL})$ and washed with $\mathrm{H}_{2} \mathrm{O}(3 \times 20 \mathrm{~mL})$. The organic phase was dried over $\mathrm{MgSO}_{4}$ and concentrated in vacuo. Flash chromatography on silica with hexanes:EtOAc (9:1) gave 5.3 as a colourless oil (14 $\mathrm{mg}, 58 \%)$.

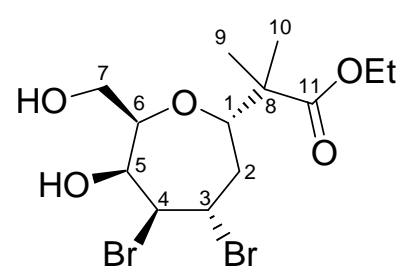

${ }^{1} \mathrm{H}$ NMR: $\delta_{\mathrm{H}}=4.51(\mathrm{dt}, J=11.0,3.7 \mathrm{~Hz}, 1 \mathrm{H}, \mathrm{H}-3), 4.43(\mathrm{t}, J=1.7$ $\mathrm{Hz}, 1 \mathrm{H}, \mathrm{H}-5), 4.34$ (dd, J=10.5, $1.0 \mathrm{~Hz}, 1 \mathrm{H}, \mathrm{H}-1), 4.25$ (dd, $J=10.7,1.9 \mathrm{~Hz}, 1 \mathrm{H}, \mathrm{H}-4), 4.18\left(\mathrm{t}, J=7.3 \mathrm{~Hz}, 2 \mathrm{H}, \mathrm{O}-\underline{\mathrm{CH}_{2}}-\mathrm{CH}_{3}\right)$, $3.84(\mathrm{td}, J=4.1,1.7 \mathrm{~Hz}, 1 \mathrm{H}, \mathrm{H}-6), 3.78(\mathrm{dd}, J=11.7,4.1 \mathrm{~Hz}, 1 \mathrm{H}$, H-7), 3.71 (dd, J=11.7, 4.1 Hz, 1H, H-7), 2.50 (ddd, J=15, 3.7, 1.2 Hz, 1H, H-2a), 2.23 (ddd, $J=15.1,12.2,10.5 \mathrm{~Hz}, 1 \mathrm{H}, \mathrm{H} 2 \mathrm{~b}), 1.28$ (t, J 7.1 Hz, 3H, O-CH $\left.2-\underline{\mathrm{CH}}_{3}\right), 1.18,(\mathrm{~s}, 3 \mathrm{H}$, H-9), 1.15 9s, 3H, H-10).

${ }^{13} \mathrm{C}$ NMR: $\delta_{\mathrm{C}}=177.2(\mathrm{C}-11), 78.9(\mathrm{C}-1), 76.0(\mathrm{C}-5), 74.9$ (C-6), 65.4 (C-7), $63.5(\mathrm{C}-4)$, $61.2\left(\mathrm{O}-\underline{\mathrm{CH}}_{2}-\mathrm{CH}_{3}\right), 53.7$ (C-3), 47.8 (C-8), 39.7 (C-2), 22.4 (C-9), 18.8 (C-10), 14.1 (O$\left.\mathrm{CH}_{2}-\underline{\mathrm{CH}}_{3}\right)$.

\section{2-((4aR,6S,8S,9R,9aS)-9-Bromo-2,2-di-tert-butyl-8-hydroxy-hexahydro-1,3,5-} trioxa-2-silabenzocyclohepten-6-yl)-2-methylpropionic acid ethyl ester (5.4). To a solution of oxepine $3.12(103 \mathrm{mg}, 0.26 \mathrm{mmol})$ in a miture of $1: 1 \mathrm{THF}: \mathrm{H}_{2} \mathrm{O}(2 \mathrm{~mL})$ at ambient temperature, was added NBS (55 mg, $0.31 \mathrm{mmol}, 1.2 \mathrm{eq}$ ) with vigorous stirring. After $1 \mathrm{~h}, \mathrm{H}_{2} \mathrm{O}(2 \mathrm{~mL})$ was added and the mixture stirred a further 5 minutes. The resulting mixture was poured onto $\mathrm{Et}_{2} \mathrm{O}(20 \mathrm{~mL})$ and washed with $\mathrm{H}_{2} \mathrm{O}(2 \times 20$ $\mathrm{mL}$ ). The organic phase was dried over $\mathrm{MgSO}_{4}$ and concentrated in vacuo. Flash 
chromatography on silica with hexanes:EtOAc (9:1) gave 5.4 as a colourless oil (99 mg, $77 \%$ ) in a 4:1 mix of isomers by NMR.

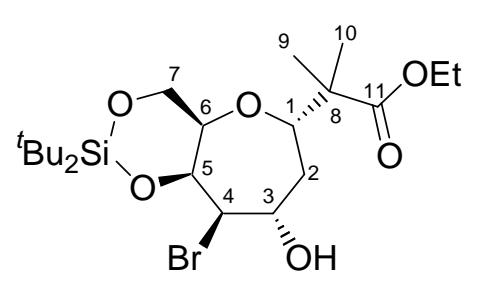

${ }^{1} \mathrm{H}$ NMR major isomer: $\delta_{\mathrm{H}}=4.50(\mathrm{~s}, 1 \mathrm{H}, \mathrm{H}-5), 4.25-4.0(\mathrm{~m}$, 7H, H-1, H-3, H-4, H-7, O- $\left.\underline{\mathrm{CH}}_{2}-\mathrm{CH}_{3}\right), 3.80$ (s, 1H, H-6), $2.39(\mathrm{~s}, 1 \mathrm{H},-\mathrm{OH}), 2.18(\mathrm{dd}, J=15.6,3.2 \mathrm{~Hz}, 1 \mathrm{H}, \mathrm{H}-2 \mathrm{a})$, 1.83 (m, 1H, H-2b), 1.25 (t, J=7.1 Hz, 3H, O- $\left.\mathrm{CH}_{2}-\underline{\mathrm{CH}}_{3}\right), 1.18$ (s, 3H, H-9), 1.15 (s, 3H, H-10), 1.07 (s, 9H, H- $\left.{ }^{t} \mathrm{Bu}-\underline{\mathrm{CH}}_{3}\right), 1.05$ (s, 9H, $\left.{ }^{-}{ }^{t} \mathrm{Bu}-\underline{\mathrm{CH}}_{3}\right)$.

${ }^{13} \mathrm{C}$ NMR: $\delta_{\mathrm{C}}=176.1(\mathrm{C}-8), 77.7(\mathrm{C}-1), 74.5$ (C-5), $71.3(\mathrm{C}-6), 70.7$ (C-7), $69.8(\mathrm{C}-3)$, $67.6(\mathrm{C}-4), 60.7\left(\mathrm{O}-\underline{\mathrm{CH}}_{2}-\mathrm{CH}_{3}\right), 48.0(\mathrm{C}-8), 33.2(\mathrm{C}-2), 27.6\left(\mathrm{C}-{ }^{t} \mathrm{Bu}-\underline{\mathrm{CH}}_{3}\right), 27.5\left(\mathrm{C}-{ }^{t} \mathrm{Bu}-\right.$ $\left.\underline{\mathrm{CH}}_{3}\right), 21.8$ (C-9), 21.5 (C-10), 20.7 ( $\left.\mathrm{Si}-\mathrm{C}\right), 14.1\left(\mathrm{O}-\mathrm{CH}_{2}-\underline{\mathrm{CH}}_{3}\right)$.

\section{2-((1aS,3S,4aR,8aS,8bS)-7,7-di-tert-butylhexahydro-1,4,6,8-trioxa-7-silabenzo[a] cyclopropa[c]cyclohepten-3-yl)-2-methylpropionic acid ethyl ester (5.5).}

To a solution of oxepine $3.12(230 \mathrm{mg}, 0.58 \mathrm{mmol})$ in $\mathrm{CH}_{2} \mathrm{Cl}_{2}(10 \mathrm{~mL})$ at ambient temperature was added $75 \% \mathrm{~m}-\mathrm{CPBA}(1.78 \mathrm{mg}, 0.75 \mathrm{mmol}, 1.5 \mathrm{eq})$ and the reaction stirred overnight. The resulting mixture was poured onto $\mathrm{Et}_{2} \mathrm{O}(20 \mathrm{~mL})$ and washed with $\mathrm{H}_{2} \mathrm{O}(3 \times 20 \mathrm{~mL})$. The organic phase was dried over $\mathrm{MgSO}_{4}$ and concentrated in vacuo. Flash chromatography on silica with 9:1 hexanes: EtOAc (9:1) gave $\mathbf{5 . 5}$ as a colourless oil (198 mg, 83\%).

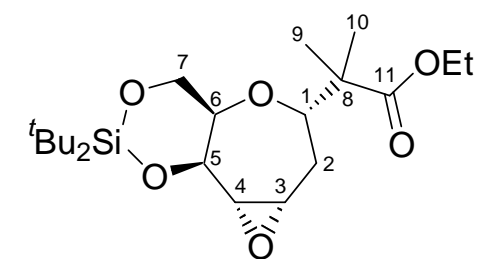

${ }^{1} \mathrm{H}$ NMR: $\delta_{\mathrm{H}}=4.60(\mathrm{~s}, 1 \mathrm{H}, \mathrm{H}-5), 4.23(\mathrm{dd}, J=12.5,1.9 \mathrm{~Hz}$, $1 \mathrm{H}, \mathrm{H}-1), 4.16$ (q, J=7.0 Hz, 2H, O- $\left.\underline{\mathrm{CH}}_{2}-\mathrm{CH}_{3}\right), 4.14$ (s, 2H, H-7), 3.58 (s, 1H, H-6), 3.28 (dt, J=8.5, 4.5 Hz, 1H, H-3), 3.05 (dd, J=4.1, $1.2 \mathrm{~Hz}, 1 \mathrm{H}, \mathrm{H}-4), 2.14$ (ddd, $J=15.9,7.8,1.9 \mathrm{~Hz}, 1 \mathrm{H}, \mathrm{H}-2 \mathrm{a}), 1.91$ (ddd, $J=15.9,12.4,5.1 \mathrm{~Hz}, 1 \mathrm{H}, \mathrm{H}-2 \mathrm{~b}), 1.24$ (t, $\left.J=7.2 \mathrm{~Hz}, 3 \mathrm{H}, \mathrm{O}-\mathrm{CH}_{2}-\underline{\mathrm{CH}}_{3}\right), 1.15$ (s, 3H, $\mathrm{H}-9), 1.08$ (s, 3H, H-10), 1.03 (s, 18H, $\left.\mathrm{H}^{t}{ }^{t} \mathrm{Bu}-\underline{\mathrm{CH}}_{3}\right)$. 
${ }^{13} \mathrm{C}$ NMR $\delta_{\mathrm{C}}=176.2(\mathrm{C}-11), 78.3(\mathrm{C}-1), 74.1(\mathrm{C}-5), 69.2(\mathrm{C}-7), 68.0(\mathrm{C}-6), 60.5(\mathrm{O}-$ $\left.\underline{\mathrm{CH}}_{2}-\mathrm{CH}_{3}\right), 56.6(\mathrm{C}-4), 55.6(\mathrm{C}-3), 47.7(\mathrm{C}-8), 27.6\left(\mathrm{C}-{ }^{t} \mathrm{Bu}-\underline{\mathrm{CH}}_{3}\right), 27.3\left(\mathrm{C}-{ }^{t}{ }^{\mathrm{Bu}}-\underline{\mathrm{CH}}_{3}\right)$, 27.1 (C-2), 23.3 (C-Si), 22.5 (C-9), 22.4 (Si-C), 20.0 (C-10), $14.2\left(\mathrm{O}-\mathrm{CH}_{2}-\underline{\mathrm{CH}}_{3}\right)$.

\section{2-((4aR,6S,8R,9S,9aR)-8-Azido-2,2-di-tert-butyl-9-hydroxy-hexahydro-1,3,5-trioxa- 2-silabenzocyclohepten-6-yl)-2-methylpropionic acid ethyl ester (5.6).}

To a solution of epoxide $\mathbf{5 . 5}(108 \mathrm{mg}, 0.26 \mathrm{mmol})$ in a mixture of 8:1 $\mathrm{MeOH}: \mathrm{H}_{2} \mathrm{O}(1$ $\mathrm{mL})$ was added $\mathrm{NaN}_{3}(89 \mathrm{mg}, 1.31 \mathrm{mmol})$ and $\mathrm{NH}_{4} \mathrm{Cl}(31 \mathrm{mg}, 0.57 \mathrm{mmol})$ and the mixture heated at reflux overnight. The mixture was poured onto $\mathrm{Et}_{2} \mathrm{O}(30 \mathrm{~mL})$, washed with $\mathrm{H}_{2} \mathrm{O}(2 \times 20 \mathrm{~mL})$ and saturated brine $(1 \times 20 \mathrm{~mL})$ and dried over $\mathrm{MgSO}_{4}$. Concentration in vacuo followed by flash chromatography on silica in hexanes:EtOAc (9:1) gave $\mathbf{5 . 6}$ as a colourless oil (76 $\mathrm{mg}, 63 \%)$.

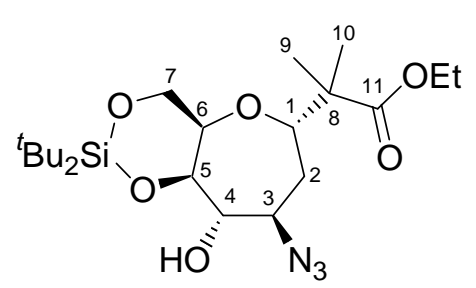

${ }^{1} \mathrm{H}$ NMR: $\delta_{\mathrm{H}}=4.25-3.95(\mathrm{~m}, 8 \mathrm{H}, \mathrm{H}-1, \mathrm{H}-3, \mathrm{H}-4, \mathrm{H}-5, \mathrm{H}-7$, O- $\left.\underline{\mathrm{CH}}_{2}-\mathrm{CH}_{3}\right), 3.8(\mathrm{~m}, 1 \mathrm{H}, \mathrm{H}-6), 3.01,(\mathrm{~s}, 1 \mathrm{H},-\mathrm{OH}), 2.05$ (ddd, $J=15.9,12.7,3.6 \mathrm{~Hz}, 1 \mathrm{H}, \mathrm{H}-2), 1.73$ (ddd, $J=15.9$, 5.2, 3.2 Hz, 1H, H-2), 1.27 (t, J=7.1 Hz, 3H, O- $\left.\mathrm{CH}_{2}-\underline{\mathrm{CH}}_{3}\right), 1.18$ (s, 3H, H-9), 1.16 (s, 3H, H-10), 1.04 (s, 9H, H- $\left.{ }^{t} \mathrm{Bu}-\underline{\mathrm{CH}}_{3}\right), 1.02$ (s, 9H, H- $\left.{ }^{t} \mathrm{Bu}-\underline{\mathrm{CH}}_{3}\right)$.

${ }^{13} \mathrm{C}$ NMR: $\delta_{\mathrm{C}}=175.9(\mathrm{C}-11), 76.9(\mathrm{C}-1), 74.5$ (C-4), 69.7 (C-3), 64.6 (C-7), 61.5 (C-6), $60.7\left(\mathrm{O}-\underline{\mathrm{CH}}_{2}-\mathrm{CH}_{3}\right), 47.4(\mathrm{C}-8), 28.5(\mathrm{C}-2), 27.4\left(\mathrm{C}-{ }^{t} \mathrm{Bu}-\underline{\mathrm{CH}}_{3}\right), 27.2\left(\mathrm{C}-{ }^{t} \mathrm{Bu}-\underline{\mathrm{CH}}_{3}\right)$, 22.1(C-9), 21.9 (C-10), 21.6 ( $\mathrm{Si}-\mathrm{C}), 21.2(\mathrm{Si}-\mathrm{C}), 14.1\left(\mathrm{O}-\mathrm{CH}_{2}-\underline{\mathrm{CH}}_{3}\right)$.

\section{2-((4aR,6S,8S,9S,9aR)-2,2-Di-tert-butyl-8,9-dihydroxyhexahydro-1,3,5-trioxa-2- silabenzocyclohepten-6-yl)-2-methylpropionic acid ethyl ester (5.7).}

To a solution of oxepine 3.12 ( $99 \mathrm{mg}, 0.25 \mathrm{mmol}$ ) in a 1:1 mix of $\mathrm{Et}_{2} \mathrm{O}: \mathrm{H}_{2} \mathrm{O}(2 \mathrm{~mL})$ at ambient temperature was added a $2.5 \%$ solution of $\mathrm{OsO}_{4}$ in water $(126 \mu \mathrm{L}, 3.2 \mathrm{mg}$, 
$0.026 \mathrm{mmol})$ and NMO $(118 \mathrm{mg}, 0.27 \mathrm{mmol})$. After $1 \mathrm{~h}$ the mixture was poured onto $\mathrm{Et}_{2} \mathrm{O}(20 \mathrm{~mL})$, washed with $\mathrm{H}_{2} \mathrm{O}(20 \mathrm{~mL})$ and dried over $\mathrm{MgSO}_{4}$. Concentration in vacuo followed by flash chromatography on silica in hexanes:EtOAc (9:1) gave $\mathbf{5 . 7}$ as a colourless oil (64 mg, 59\%).

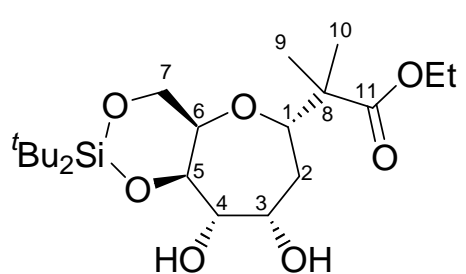

${ }^{1} \mathrm{H}$ NMR: $\delta_{\mathrm{H}}=4.15-3.95(\mathrm{~m}, 8 \mathrm{H}, \mathrm{H}-1, \mathrm{H}-3, \mathrm{H}-4, \mathrm{H}-5, \mathrm{H}-7$, O- $\left.\underline{\mathrm{CH}}_{2}-\mathrm{CH}_{3}\right), 3.80$ (s, 1H, H-6), 2.7 (bs, $\left.2 \mathrm{H},-\mathrm{O} \underline{\mathrm{H}}\right), 2.34$ (m, 1H, H-2a), 1.51 (dd, J=14.4, $1.3 \mathrm{~Hz}, 1 \mathrm{H}, \mathrm{H}-2 \mathrm{~b}), 1.19$ (t, $\left.J=7.1 \mathrm{~Hz}, 3 \mathrm{H}, \mathrm{O}-\mathrm{CH}_{2}-\underline{\mathrm{CH}}_{3}\right), 1.12$ (s, 3H, H-9), 1.07 (s, 3H, H-10), 0.96 (s, 9H, H- ${ }^{t} \mathrm{Bu}-$ $\left.\underline{\mathrm{CH}}_{3}\right), 0.95\left(\mathrm{~s}, 9 \mathrm{H}, \mathrm{H}^{-}{ }^{t} \mathrm{Bu}-\underline{\mathrm{CH}}_{3}\right)$.

${ }^{13} \mathrm{C}$ NMR: $\delta_{\mathrm{C}}=176.6(\mathrm{C}-8), 78.3(\mathrm{C}-1), 75.5(\mathrm{C}-3), 75.0(\mathrm{C}-4), 70.1(\mathrm{C}-7), 70.0(\mathrm{C}-5)$, $68.2(\mathrm{C}-6), 60.7\left(\mathrm{O}-\underline{\mathrm{CH}}_{2}-\mathrm{CH}_{3}\right), 47.9(\mathrm{C}-8), 28.3(\mathrm{C}-2), 27.6\left(\mathrm{C}-{ }^{t} \mathrm{Bu}-\underline{\mathrm{CH}}_{3}\right), 27.5\left(\mathrm{C}-{ }^{t} \mathrm{Bu}-\right.$ $\left.\underline{\mathrm{CH}}_{3}\right), 21.9$ (C-9), 21.2 (C-10), 20.7 (Si-C), $14.1\left(\mathrm{O}-\mathrm{CH}_{2}-\underline{\mathrm{CH}}_{3}\right)$.

\section{2-((4aR,6S,9R,9aR)-2,2-Di-tert-butyl-9-hydroxyhexahydro-1,3,5-trioxa-2-silabenzo cyclohepten-6-yl)-2-methylpropionic acid ethyl ester (5.8).}

To a solution of oxepine $3.12(78 \mathrm{mg}, 0.20 \mathrm{mmol})$ in $\mathrm{THF}(1 \mathrm{~mL})$ at $0{ }^{\circ} \mathrm{C}$ was added $2 \mathrm{M} \mathrm{BH}_{3}$-DMS complex $(198 \mu \mathrm{L}, 0.39 \mathrm{mmol})$. After stirring $1 \mathrm{~h}$ at $0{ }^{\circ} \mathrm{C}$ the reaction was quenched by the addition of $\mathrm{H}_{2} \mathrm{O}(100 \mu \mathrm{L})$ and stirred for $5 \mathrm{~min}$. To the mixture was added $3 \mathrm{M} \mathrm{NaOH}(0.2 \mathrm{~mL})$ and $3 \mathrm{M} \mathrm{H}_{2} \mathrm{O}_{2}(0.2 \mathrm{~mL})$. This was warmed to ambient temperature and stirred overnight. The mixture was poured onto $\mathrm{Et}_{2} \mathrm{O}(20 \mathrm{~mL})$, washed with $\mathrm{H}_{2} \mathrm{O}(2 \times 20 \mathrm{~mL})$ and dried over $\mathrm{MgSO}_{4}$. Concentration in vacuo followed by flash chromatography on silica in hexanes:EtOAc (9:1) gave $\mathbf{5 . 8}$ as a colourless oil (32 $\mathrm{mg}, 40 \%)$ and recovered starting material (20 mg, 26\%) 
${ }^{1} \mathrm{H}$ NMR: $\delta_{\mathrm{H}}=4.2-3.95(\mathrm{~m}, 7 \mathrm{H}, \mathrm{H}-4, \mathrm{H}-5, \mathrm{H}-6, \mathrm{H}-7, \mathrm{O}-$

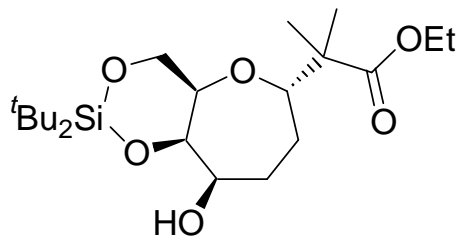

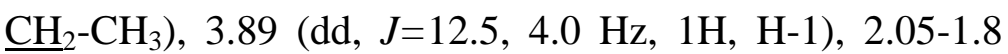

(m, 3H, H-2, H-3), 1.48 (m, 1H, H-3), 1.25 (t, J=7.1 Hz, $\left.3 \mathrm{H}, \mathrm{O}-\mathrm{CH}_{2}-\underline{\mathrm{CH}}_{3}\right), 1.18$ (s, 3H, H-9), 1.12 (s, 3H, H-10), 1.03 (s, 9H, H- ${ }^{t} \mathrm{Bu}-\underline{\mathrm{CH}}_{3}$ ), 1.02 (s, $\left.9 \mathrm{H}, \mathrm{H}-{ }^{t} \mathrm{Bu}-\underline{\mathrm{CH}}_{3}\right)$.

${ }^{13} \mathrm{C}$ NMR: $\delta_{\mathrm{C}}=176.7(\mathrm{C}-11), 81.7(\mathrm{C}-1), 78.6(\mathrm{C}-6), 70.9$ (C-5), 69.7 (C-7), 68.8 (C-4), $60.5\left(\mathrm{O}-\underline{\mathrm{CH}}_{2}-\mathrm{CH}_{3}\right), 48.1(\mathrm{C}-8), 28.0(\mathrm{C}-2), 27.5\left(\mathrm{C}-{ }^{t} \mathrm{Bu}-\underline{\mathrm{CH}}_{3}\right), 23.2\left(\mathrm{C}-{ }^{t} \mathrm{Bu}-\underline{\mathrm{CH}}_{3}\right)$,

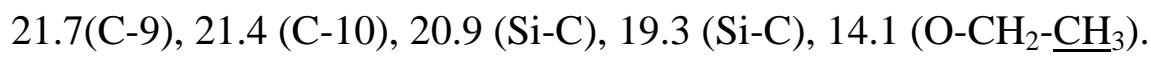

\section{2-((4aR,6S,9aR)-Di-tert-butyl-4a,6,7,9a-tetrahydro-4H-1,3,5-trioxa-2-silabenzo} cyclohepten-6-yl)-2-methylpropan-1-ol (5.9).

To a solution of the oxepine $\mathbf{3 . 1 2}(65 \mathrm{mg}, 0.163 \mathrm{mmol})$ in $\mathrm{CH}_{2} \mathrm{Cl}_{2}(1 \mathrm{~mL})$ at $-78{ }^{\circ} \mathrm{C}$ was added 1M DIBAL in $\mathrm{CH}_{2} \mathrm{Cl}_{2}(163 \mu \mathrm{L}, 0.163 \mathrm{mmol})$. After stirring for $2 \mathrm{~h}, \mathrm{H}_{2} \mathrm{O}(500$ $\mu \mathrm{L}$ ) was added and the reaction warmed to ambient temperature. The mixture was poured onto $\mathrm{Et}_{2} \mathrm{O}(20 \mathrm{~mL})$, washed with $\mathrm{H}_{2} \mathrm{O}(2 \times 20 \mathrm{~mL})$ and dried over $\mathrm{MgSO}_{4}$. Concentration in vacuo followed by flash chromatography on silica in hexanes:EtOAc (9:1) gave 5.9 as a colourless oil $(13 \mathrm{mg}, 54 \%)$ and recovered starting material (15 mg, $23 \%)$.

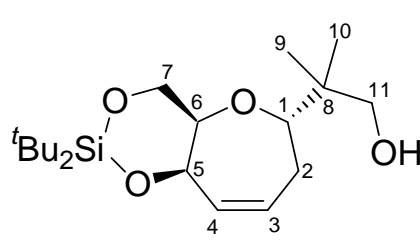

${ }^{1} \mathrm{H}$ NMR: $\delta_{\mathrm{H}}=5.87(\mathrm{~m}, 1 \mathrm{H}, \mathrm{H}-3), 5.74(\mathrm{ddd}, J=11.2,4.4,2.6$ $\mathrm{Hz}, 1 \mathrm{H}, \mathrm{H}-4), 4.85$ (m, 1H, H-5), 4.26 (s, 2H, H-11), 4.06 (s, 1H, H-6), 4.01 (dd, $J=11.5,1.7 \mathrm{~Hz}, 1 \mathrm{H}, \mathrm{H}-1), 3.56$ (d, $J=9.0$ Hz, 1H, H-7a), 3.37 (d, J=9.0 Hz, 1H, H-7b), 2.52 (dddd, J=16.4, 10.5, 5.4, $2.7 \mathrm{~Hz}, 1 \mathrm{H}$, H-2a), 2.14 (ddd, J=16.9, 9.1, $1.7 \mathrm{~Hz}, 1 \mathrm{H}, \mathrm{H}-2 \mathrm{~b}), 1.08$ (s, 9H, H- ${ }^{t} \mathrm{Bu}-\underline{\mathrm{CH}}_{3}$ ), 1.07 (s, 9H, $\left.\mathrm{H}-{ }^{t} \mathrm{Bu}-\underline{\mathrm{CH}}_{3}\right), 0.97$ (s, 3H, H-9), 0.82 (s, 3H, H-10). 
${ }^{13} \mathrm{C}$ NMR: $\delta_{\mathrm{C}}=132.1(\mathrm{C}-4), 128.0(\mathrm{C}-3), 86.1(\mathrm{C}-1), 75.0(\mathrm{C}-5), 72.8(\mathrm{C}-7), 72.2(\mathrm{C}-6)$, 68.7 (C-11), 39.0 (C-8), $27.7\left(\mathrm{C}-{ }^{t} \mathrm{Bu}-\underline{\mathrm{CH}}_{3}\right), 27.2\left(\mathrm{C}-{ }^{t} \mathrm{Bu}-\underline{\mathrm{CH}}_{3}\right), 25.7$ (C-2), 22.7 (C-9), $20.6(\mathrm{C}-10), 18.2(\mathrm{Si}-\mathrm{C})$.

General method for acetylation of oxepanes: to the oxepane (1 eq.) in $\mathrm{CH}_{2} \mathrm{Cl}_{2}$ (1 mL) was added $\mathrm{Ac}_{2} \mathrm{O}$ (2 eq. per hydroxyl), py (3 eq. per hydroxyl) and a catalytic amount of DMAP. The reaction was stirred till complete by TLC, poured onto $\mathrm{Et}_{2} \mathrm{O}(10 \mathrm{~mL})$ and washed with $\mathrm{H}_{2} \mathrm{O}(3 \times 10 \mathrm{~mL})$. The mixture was dried over $\mathrm{MgSO}_{4}$ and concentrated in vacuo followed by flash chromatography on silica with hexanes: EtOAc (25:1) to give the acetylated oxepane product.

2-((4aR,6S,8S,9R,9aS)-8-Acetoxy-9-bromo-2,2-di-tert-butylhexahydro-1,3,5-trioxa2-silabenzocyclohepten-6-yl)-2-methylpropionic acid ethyl ester (5.10).

Bromohydrin $5.4(54 \mathrm{mg}, 0.11 \mathrm{mmol})$ was acetylated using the general procedure to give 5.10 as a clear oil $(50 \mathrm{mg}, 85 \%)$.

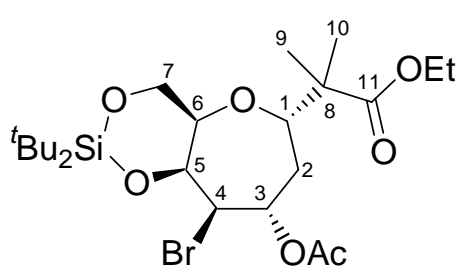

${ }^{1} \mathrm{H}$ NMR: $\delta_{\mathrm{H}}=5.14(\mathrm{td}, J=10.4,1.6 \mathrm{~Hz}, 1 \mathrm{H}, \mathrm{H}-3), 4.53(\mathrm{~d}$, $1 \mathrm{H}, J=3.3 \mathrm{~Hz}, 1 \mathrm{H}, \mathrm{H}-5), 4.2-4.0$ (m, 6H, H-1, H-4, H-7, O$\left.\underline{\mathrm{CH}_{2}}-\mathrm{CH}_{3}\right), 3.80$ (s, 1H, H-6), 2.09 (s, 3H, H-Ac- $\left.\underline{\mathrm{CH}}_{3}\right), 1.96$ (m, 2H, H-2), $1.24\left(\mathrm{t}, 3 \mathrm{H}, J=7.0 \mathrm{~Hz}, 3 \mathrm{H}, \mathrm{O}-\mathrm{CH}_{2}-\underline{\mathrm{CH}}_{3}\right), 1.15(\mathrm{~s}, 3 \mathrm{H}, \mathrm{H}-9), 1.12(\mathrm{~s}, 3 \mathrm{H}$, $\mathrm{H}-10), 1.06$ (s, 18H, H- $\left.{ }^{t} \mathrm{Bu}-\underline{\mathrm{CH}}_{3}\right)$.

${ }^{13} \mathrm{C}$ NMR: $\delta_{\mathrm{C}}=176.0(\mathrm{C}-11), 169.6$ (C-Ac-CO), $78.0(\mathrm{C}-1), 74.3(\mathrm{C}-5), 72.1(\mathrm{C}-3), 70.5$ (C-7), $60.8\left(\mathrm{O}-\underline{\mathrm{CH}}_{2}-\mathrm{CH}_{3}\right), 58.7(\mathrm{C}-4), 47.8(\mathrm{C}-8), 33.3(\mathrm{C}-2), 27.6\left(\mathrm{C}-{ }^{t} \mathrm{Bu}-\underline{\mathrm{CH}}_{3}\right), 23.7$ (Si-C), 21.7 (C-9), 21.0 (C-10), $20.7\left(\mathrm{C}-\mathrm{Ac}-\underline{\mathrm{CH}}_{3}\right), 14.1\left(\mathrm{O}-\mathrm{CH}_{2}-\underline{\mathrm{CH}}_{3}\right)$.

ES HRMS $m / z$ : calculated for $\mathrm{C}_{23} \mathrm{H}_{42} \mathrm{O}_{7} \mathrm{BrSi}+\mathrm{NH}_{4}$ 554.21485. Found 554.75042. 
2-silabenzocyclohepten-6-yl)-2-methylpropionic acid ethyl ester (5.11).

Aza alcohol 5.6 (37 mg, $0.08 \mathrm{mmol})$ was acetylated using the general procedure to give $\mathbf{5 . 1 1}$ as a clear oil (28 $\mathrm{mg}, 70 \%)$.

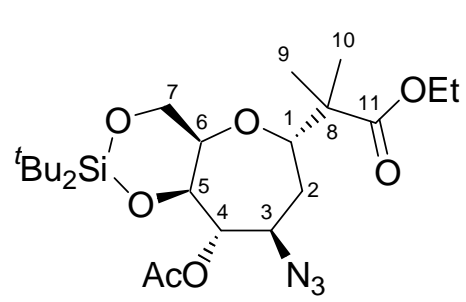

${ }^{1} \mathrm{H}$ NMR: $\delta_{\mathrm{H}}=5.33(\mathrm{t}, J=6.2 \mathrm{~Hz}, 1 \mathrm{H}, \mathrm{H}-4), 4.2-4.05(\mathrm{~m}$, $6 \mathrm{H}, \mathrm{H}-1, \mathrm{H}-5, \mathrm{H}-7, \mathrm{O}-\underline{\mathrm{CH}}_{2}-\mathrm{CH}_{3}$ ), 3.95 (ddd, J=6.6, 5.1, 3.4 Hz, 1H, H-3), 3.88 (dd, J=6.6, 3.7 Hz, 1H, H-6), 2.17 (ddd, $J=15.7,12.8,3.4 \mathrm{~Hz}, 1 \mathrm{H}, \mathrm{H}-2 \mathrm{a}), 2.10$ (s, 3H, H-Ac- $\left.\underline{\mathrm{H}}_{3}\right)$, 1.75 (ddd, J=15.7, 3.7, 3.0, 1H, H-2b) 1.24 (t, 3H, J 7.1 Hz, O-CH $\left.2-\underline{C H}_{3}\right), 1.17$ (s, 3H, H-9), 1.15 (s, 3H, H-10), 1.02 (s, 9H, H- $\left.{ }^{t} \mathrm{Bu}-\underline{\mathrm{CH}}_{3}\right), 1.00$ (s, 9H, H- $\left.{ }^{t} \mathrm{Bu}-\underline{\mathrm{CH}}_{3}\right)$.

${ }^{13} \mathrm{C}$ NMR: $\delta_{\mathrm{C}}=176.1(\mathrm{C}-11), 169.5$ (C-Ac- $\left.\underline{\mathrm{CO}}\right), 76.7$ (C-1), 75.9 (C-5), $73.7(\mathrm{C}-4), 69.3$ (C-6), $67.6(\mathrm{C}-7), 60.7\left(\mathrm{O}-\underline{\mathrm{CH}}_{2}-\mathrm{CH}_{3}\right), 58.8(\mathrm{C}-3), 47.8(\mathrm{C}-8), 27.5\left(\mathrm{C}-{ }^{t} \mathrm{Bu}-\underline{\mathrm{CH}}_{3}\right), 27.1$ (C- $\left.{ }^{t} \mathrm{Bu}-\underline{\mathrm{CH}}_{3}\right), 26.7$ (C-2), 22.9 (Si-C), $22.2(\mathrm{C}-9), 21.7$ (C-10), $14.1\left(\mathrm{O}-\mathrm{CH}_{2}-\underline{\mathrm{CH}}_{3}\right)$.

\section{2-((4aR,6S,8S,9S,9aRS)-8,9-Acetoxy-2,2-di-tert-butylhexahydro-1,3,5-trioxa-2-sila} benzocyclohepten-6-yl)-2-methylpropionic acid ethyl ester (5.12)

Diol $5.7(17 \mathrm{mg}, 0.04 \mathrm{mmol})$ was acetylated using the general procedure to give $\mathbf{5 . 1 2}$ as a clear oil (16 mg, 79\%).

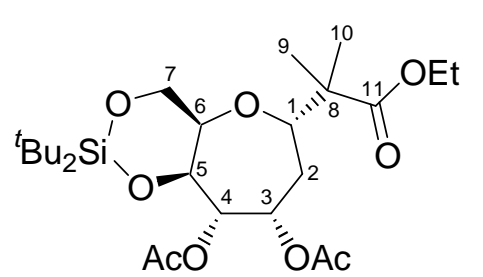

${ }^{1} \mathrm{H}$ NMR: $\delta_{\mathrm{H}}=5.24(\mathrm{~d}, J=1.7 \mathrm{~Hz}, 1 \mathrm{H}, \mathrm{H}-4), 5.19(\mathrm{~d}, J=10.5$ $\mathrm{Hz}, 1 \mathrm{H}, \mathrm{H}-3), 4.2-4.05$ (m, 5H, H-1, H-7, O- $\underline{\mathrm{CH}}_{2}-\mathrm{CH}_{3}$ ), 3.99 (d, J=3.7 Hz, 1H, H-5), 3.81 (s, 1H, H-6), 2.49 (td, $\mathrm{J}=12.5,10.5 \mathrm{~Hz}, 1 \mathrm{H}, \mathrm{H}-2 \mathrm{a}$ ), 2.14 (s, 3H, H-Ac- $\underline{\mathrm{CH}}_{3}$ ), 2.02 (s, 3H, H-Ac- $\underline{\mathrm{CH}}_{3}$ ), 1.67 (dd, $J=13.4,3.0 \mathrm{~Hz}, \mathrm{H}-2 \mathrm{~b}), 1.27$ (t, J=7.1 Hz, 3H, O- $\mathrm{CH}_{2}-\underline{\mathrm{CH}}_{3}$ ), 1.19 (s, 3H, H-9), 1.17 (s, 3H, H-10), 1.03 (s, 9H, H- ${ }^{t} \mathrm{Bu}-\underline{\mathrm{CH}}_{3}$ ), 1.01 (s, 9H, H- $\left.{ }^{t} \mathrm{Bu}-\underline{\mathrm{CH}}_{3}\right)$.

${ }^{13} \mathrm{C}$ NMR: $\delta_{\mathrm{C}}=176.1(\mathrm{C}-11), 169.9$ (C-Ac-므), 167.7 (C-Ac므), 78.5 (C-1), 74.0 (C4), 73.5 (C-5), 70.6 (C-3), 70.4 (C-7), 68.6 (C-6), $60.7\left(\mathrm{O}-\underline{\mathrm{CH}}_{2}-\mathrm{CH}_{3}\right), 47.7$ (C-8), 27.5 
(C- $\left.{ }^{t} \mathrm{Bu}-\underline{\mathrm{CH}}_{3}\right), 27.4\left(\mathrm{C}-{ }^{t} \mathrm{Bu}-\underline{\mathrm{CH}}_{3}\right), 27.0(\mathrm{C}-2), 23.5(\mathrm{Si}-\mathrm{C}), 22.4(\mathrm{Si}-\mathrm{C}), 21.5(\mathrm{C}-9), 21.2$ (C-10), $21.1\left(\mathrm{C}-\mathrm{Ac}-\underline{\mathrm{CH}}_{3}\right), 20.8(\mathrm{C}-\mathrm{Ac}-\underline{\mathrm{CH}} 3), 14.1\left(\mathrm{O}-\mathrm{CH}_{2}-\underline{\mathrm{CH}}_{3}\right)$.

2-((4aR,6S,9R,9aR)-9-Acetoxy-2,2-di-tert-butylhexahydro-1,3,5-trioxa-2-silabenzo cyclohepten-6-yl)-2-methylpropionic acid ethyl ester (5.13).

Alcohol $5.8(28 \mathrm{mg}, 0.07 \mathrm{mmol})$ was acetylated using the general procedure to give $\mathbf{5 . 1 3}(19 \mathrm{mg}$, 68\%).

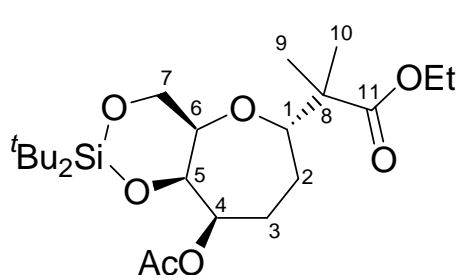

${ }^{1} \mathrm{H}$ NMR: $\delta_{\mathrm{H}}=4.96(\mathrm{t}, J=3.4 \mathrm{~Hz}, 1 \mathrm{H}, \mathrm{H}-4), 4.2-4.0(\mathrm{~m}, 5 \mathrm{H}$, $\left.\mathrm{H}-5, \mathrm{H}-7, \mathrm{O}-\underline{\mathrm{CH}}_{2}-\mathrm{CH}_{3}\right), 3.89$ (dd, J=12.5, $4.4 \mathrm{~Hz}, 1 \mathrm{H}, \mathrm{H}-$ 1), $3.82(\mathrm{t}, J=2.0 \mathrm{~Hz}, 1 \mathrm{H}, \mathrm{H}-6), 2.08$ (s, 3H, H-Ac- $\left.\underline{\mathrm{CH}}_{3}\right)$, $2.00(\mathrm{~m}, 1 \mathrm{H}, \mathrm{H}-2), 1.92(\mathrm{~m}, 2 \mathrm{H}, \mathrm{H}-3), 1.51(\mathrm{~m}, 1 \mathrm{H}, \mathrm{H}-2), 1.26(\mathrm{t}, J=7.0 \mathrm{~Hz}, 3 \mathrm{H}, \mathrm{O}-$ $\left.\mathrm{CH}_{2}-\underline{\mathrm{CH}}_{3}\right), 1.19$ (s, 3H, H-9), 1.15 (s, 3H, H-10), 1.03 (s, 9H, H- $\left.{ }^{t} \mathrm{Bu}-\underline{\mathrm{CH}}_{3}\right), 1.02$ (s, 9H, $\left.\mathrm{H}-{ }^{t} \mathrm{Bu}-\underline{\mathrm{CH}}_{3}\right)$.

${ }^{13} \mathrm{C}$ NMR: $\delta_{\mathrm{C}}=176.6(\mathrm{C}-11), 169.7(\mathrm{C}-\mathrm{Ac}-\mathrm{CO}), 82.4(\mathrm{C}-1), 75.7$ (C-5), $73.3(\mathrm{C}-4), 70.6$ (C-7), $68.9(\mathrm{C}-6), 60.5\left(\mathrm{O}-\underline{\mathrm{CH}}_{2}-\mathrm{CH}_{3}\right), 48.0(\mathrm{C}-8), 27.6\left(\mathrm{C}-{ }^{t} \mathrm{Bu}-\underline{\mathrm{CH}}_{3}\right), 27.4\left(\mathrm{C}-{ }^{t} \mathrm{Bu}-\underline{\mathrm{CH}}_{3}\right)$, 25.5 (C-3), 22.0 (Si-C), 21.7 (C-9), 21.3 (C-10), 20.8 (C-Ac- $\underline{\mathrm{CH}}_{3}$ ), 19.7 (C-2), 14.1 (O$\left.\mathrm{CH}_{2}-\underline{\mathrm{CH}}_{3}\right)$.

\section{$(1 \mathrm{a} R, 2 \mathrm{a} S, 6 \mathrm{a} R, 7 \mathrm{a} S)-4,4-D i-t e r t-b u t y l t e t r a h y d r o-3,5,7-t r i o x a-4-s i l a c y c l o p r o p a[b]$} naphthalene-2-one (6.3).

To a solution of oxalyl chloride $(490 \mu \mathrm{L}, 5.66 \mathrm{mmol})$ in $\mathrm{CH}_{2} \mathrm{Cl}_{2}(22 \mathrm{~mL})$ at $-78{ }^{\circ} \mathrm{C}$ was added dropwise DMSO (800 $\mu \mathrm{L}, 11.33 \mathrm{mmol})$ and stirred for $15 \mathrm{~min}$. Cyclopropane 3.3 (1.417 g, $4.72 \mathrm{mmol})$ in $\mathrm{CH}_{2} \mathrm{Cl}_{2}(8 \mathrm{~mL})$ was added dropwise and the mixture stirred for 30 minutes. $\mathrm{Et}_{3} \mathrm{~N}(2.63 \mathrm{~mL}, 18.88 \mathrm{mmol})$ was then added dropwise and the mixture was stirred for $90 \mathrm{~min}$. The mixture was warmed to ambient temperature, poured onto saturated aq $\mathrm{NaHCO}_{3}(30 \mathrm{~mL})$ and extracted with $\mathrm{CH}_{2} \mathrm{Cl}_{2}(2 \times 30 \mathrm{~mL})$. The combined 
organic layers were then washed with saturated aq $\mathrm{Na}_{2} \mathrm{SO}_{3}(2 \times 30 \mathrm{~mL})$, saturated brine (3 x $30 \mathrm{~mL}$ ), dried over $\mathrm{MgSO}_{4}$ and concentrated in vacuo to give 6.3 as a white solid (1.325 g, 94\%) which was considered pure enough by ${ }^{1} \mathrm{H}$ NMR to use without purification.<smiles>CC(C)C[SiH2]OC1C(=O)C2CC2O[C@@H]2COCC12</smiles>

${ }^{1} \mathrm{H}$ NMR: $\delta_{\mathrm{H}}=4.36(\mathrm{~s}, 1 \mathrm{H}, \mathrm{H}-4), 4.25(\mathrm{~d}, J=1.5 \mathrm{~Hz}, 1 \mathrm{H}, \mathrm{H}-6 \mathrm{a})$, $4.22(\mathrm{~d}, J=2.2 \mathrm{~Hz}, 1 \mathrm{H}, \mathrm{H}-6 \mathrm{~b}), 4.18(\mathrm{td}, J=5.4,3.2 \mathrm{~Hz}, 1 \mathrm{H}, \mathrm{H}-1)$, 3.79 (s, 1H, H-5), 1.99 (td, J=5.9, 3.4 Hz, 1H, H-1'a), 1.74 (m, 1H, H-2), 1.31 (m, 1H, H-1'b), 1.03 (s, $\left.18 \mathrm{H}, \mathrm{H}^{-}{ }^{t} \mathrm{Bu}-\underline{\mathrm{CH}}_{3}\right)$.

${ }^{13} \mathrm{C}$ NMR: $\delta_{\mathrm{C}}=200.6(\mathrm{C}-3), 77.9(\mathrm{C}-5), 77.4(\mathrm{C}-4), 66.9(\mathrm{C}-6), 57.6(\mathrm{C}-1), 27.5\left(\mathrm{C}-{ }^{t} \mathrm{Bu}-\right.$ $\left.\underline{\mathrm{CH}}_{3}\right), 27.4\left(\mathrm{C}-{ }^{t} \mathrm{Bu}-\underline{\mathrm{CH}}_{3}\right), 23.4(\mathrm{C}-2), 22.0$ (Si-C), 20.7 (Si-C), 18.2 (C-1').

IR: $2935,2859,1700,1203,1130,912,733,650 \mathrm{~cm}^{-1}$.

$\operatorname{MP} 112^{\circ}$

\section{$(1 S, 3 R, 4 R, 5 R, 6 R)-6$-(tert-Butyldiphenylsilanyloxymethyl)-5-methyl-2-oxa-bicyclo}

\section{[4.1.0]heptane-4,5-diol (6.4).}

To a solution of ketone $6.3(1.325 \mathrm{~g}, 4.43 \mathrm{mmol})$ in THF $(20 \mathrm{~mL})$ at $0{ }^{\circ} \mathrm{C}$ was added 3 $\mathrm{M} \mathrm{MeMgBr}$ in hexanes $(2.27 \mathrm{~mL}, 6.81 \mathrm{mmol})$. The mixture was allowed to warm to ambient temperature and stirred for $4 \mathrm{~h}$. The reaction mixture was diluted with $\mathrm{Et}_{2} \mathrm{O}$ (50 mL) and washed with $\mathrm{H}_{2} \mathrm{O}(3 \times 30 \mathrm{~mL})$. The organic phase was dried over $\mathrm{MgSO}_{4}$ and concentrated in vacuo. Flash chromatography on silica gel with hexanes: EtOAc (5:1) gave 6.4 as a colourless oil $(1.20 \mathrm{~g}, 86 \%)$.

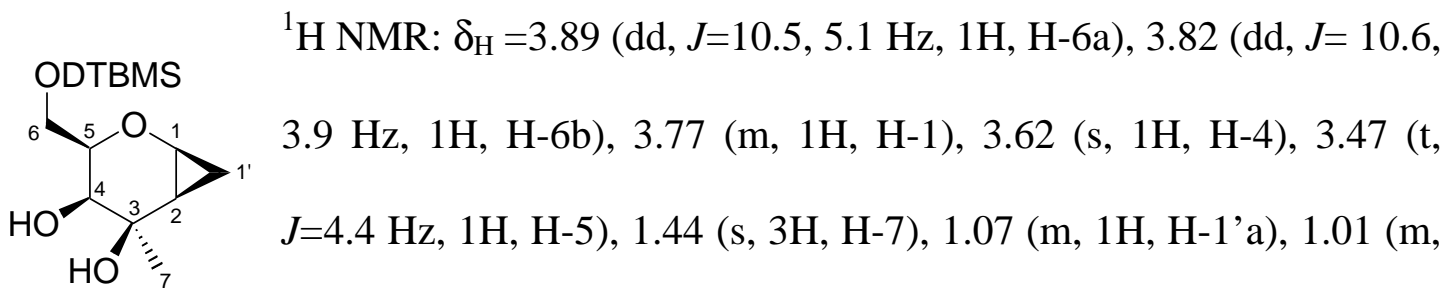

1H, H-2), 0.97 (s, 9H, H- $\left.{ }^{t} \mathrm{Bu}-\underline{\mathrm{CH}}_{3}\right), 0.96$ (s, 9H, H- $\left.{ }^{t} \mathrm{Bu}-\underline{\mathrm{CH}}_{3}\right), 0.65$ (dt, J=10.0, $5.8 \mathrm{~Hz}$, 1H, H-1'b), 0.10 (s, 3H, Si- $\left.\underline{\mathrm{CH}}_{3}\right)$. 
${ }^{13} \mathrm{C}$ NMR: $\delta_{\mathrm{C}}=75.2(\mathrm{C}-5), 74.9(\mathrm{C}-4), 67.5(\mathrm{C}-3), 65.5(\mathrm{C}-6), 54.3(\mathrm{C}-1), 27.7$ (C-C-7), $27.6\left(\mathrm{C}-{ }^{t} \mathrm{Bu}-\underline{\mathrm{CH}}_{3}\right), 27.5\left(\mathrm{C}-{ }^{t} \mathrm{Bu}-\underline{\mathrm{CH}}_{3}\right), 22.6$ (C-2), 20.7 (Si-C), 20.6 (Si-C), 12.1 (C-1'), $-9.5\left(\mathrm{Si}-\underline{\mathrm{CH}}_{3}\right)$.

ES HRMS m/z: calculated for $\mathrm{C}_{17} \mathrm{H}_{34} \mathrm{O}_{4} \mathrm{Si}-\mathrm{H} 329.2154$. Found 329.2141. IR: $2934,1254,910,735,648 \mathrm{~cm}^{-1}$.

\section{$(1 S, 3 R, 4 S, 5 R, 6 R)-6$-(tert-Butyldiphenylsilanyloxymethyl)-5-methyl-2-oxa-bicyclo}

\section{[4.1.0]heptane-4,5-diol (6.8).}

To a solution of ketone $3.20(0.98 \mathrm{~g}, 3.28 \mathrm{mmol})$ in $\mathrm{THF}(40 \mathrm{~mL})$ at $0{ }^{\circ} \mathrm{C}$ was added 3 $\mathrm{M} \mathrm{MeMgI}$ in $\mathrm{Et}_{2} \mathrm{O}(2.19 \mathrm{~mL}, 6.57 \mathrm{mmol})$. The mixture was allowed to warm to ambient temperature and stirred for $4 \mathrm{~h}$. The reaction mixture was poured onto $\mathrm{Et}_{2} \mathrm{O}$ (50 mL) and washed with $\mathrm{H}_{2} \mathrm{O}(3 \times 30 \mathrm{~mL})$. The organic phase was dried over $\mathrm{MgSO}_{4}$ and concentrated in vacuo. Flash chromatography on silica gel with hexanes:EtOAc (5:1) gave diol 6.8 as a colourless oil (1.036 g, 99\%).

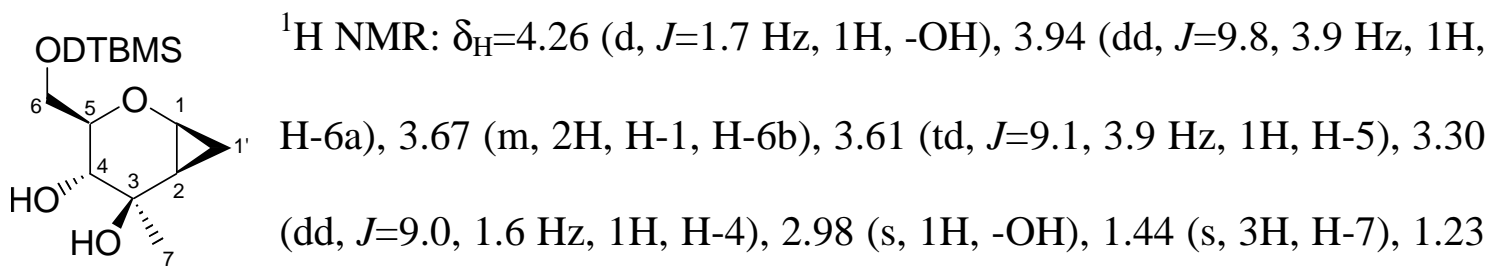
(m, 1H, H-2), 0.97 (s, 9H, H- $\left.{ }^{t} \mathrm{Bu}-\underline{\mathrm{CH}}_{3}\right), 0.96$ (s, 9H, H- $\left.{ }^{t} \mathrm{Bu}-\underline{\mathrm{CH}}_{3}\right), 0.72$ (dt, J=11.0, 6.3 Hz, 1H, H-1'a), 0.29 (m, 1H, H-1'b), 0.10 (s, 3H, H-Si- $\underline{\mathrm{CH}}_{3}$ ).

${ }^{13} \mathrm{C}$ NMR: $\delta_{\mathrm{C}}=76.0(\mathrm{C}-5), 70.3(\mathrm{C}-4), 68.6(\mathrm{C}-6), 67.1(\mathrm{C}-3), 52.1(\mathrm{C}-1), 27.6\left(\mathrm{C}-{ }^{t} \mathrm{Bu}-\right.$ $\left.\underline{\mathrm{CH}}_{3}\right), 27.5\left(\mathrm{C}-{ }^{t} \mathrm{Bu}-\underline{\mathrm{CH}}_{3}\right), 26.0$ (C-7), 23.1 (C-2), 20.7 (Si-C), 20.5 (Si-C), 11.5 (C-1'), $9.6\left(\mathrm{Si}-\underline{\mathrm{CH}}_{3}\right)$.

(2S)-((1S)-1,2-dihydroxy)ethyl-3-methyl-5,6-dihydro-(2H)-pyran (6.7).

To a mixture of $6.4(1.2 \mathrm{~g}, 3.81 \mathrm{mmol})$ and TESH $(3.037 \mathrm{~mL}, 19.05 \mathrm{mmol})$ in $\mathrm{MeCN}$ $(20 \mathrm{~mL})$ at $0{ }^{\circ} \mathrm{C}$ was added dropwise TMSOTf $(345 \mu \mathrm{L}, 1.905 \mathrm{mmol})$ and the reaction 
stirred for $2 \mathrm{~h}$. The reaction was quenched with $\mathrm{Et}_{3} \mathrm{~N}(530 \mu \mathrm{L}, 3.81 \mathrm{mmol})$ and poured onto a silica plug. The silica plug was washed with hexanes $(100 \mathrm{~mL})$ to remove excess TESH, and then washed with EtOAc $(2 \times 100 \mathrm{~mL})$. The EtOAc washings were concentrated in vacuo to give $\mathbf{6 . 7}$ as a colourless oil (365 mg, 60\%).

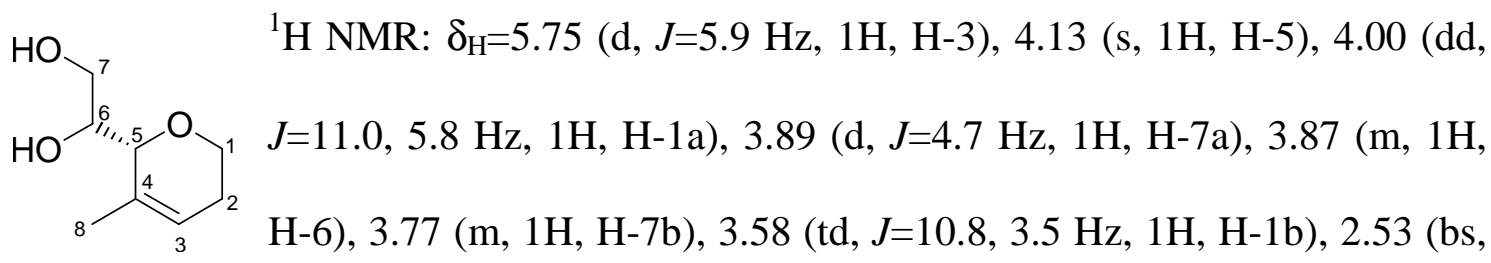
1H, -OH), 2.37 (bd, J=7.0 Hz, 1H, -OH), 2.32 (m, 1H, H-2a), 1.90 (m, 1H, H-2b), 1.70 (s, 3H, H-8).

${ }^{13} \mathrm{C}$ NMR: $\delta_{\mathrm{C}}=132.6(\mathrm{C}-4), 122.8(\mathrm{C}-3), 79.2(\mathrm{C}-5), 69.9$ (C-6), 65.5 (C-7), $63.7(\mathrm{C}-1)$, $25.1(\mathrm{C}-2), 19.0(\mathrm{C}-8)$.

ES HRMS m/z: calculated for $\mathrm{C}_{8} \mathrm{H}_{14} \mathrm{O}_{3}+\mathrm{H}$ 159.1016. Found 159.1015.

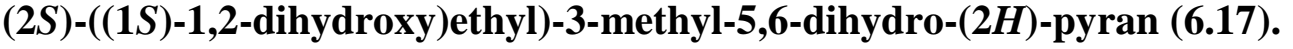

Method as described in text (page 138).

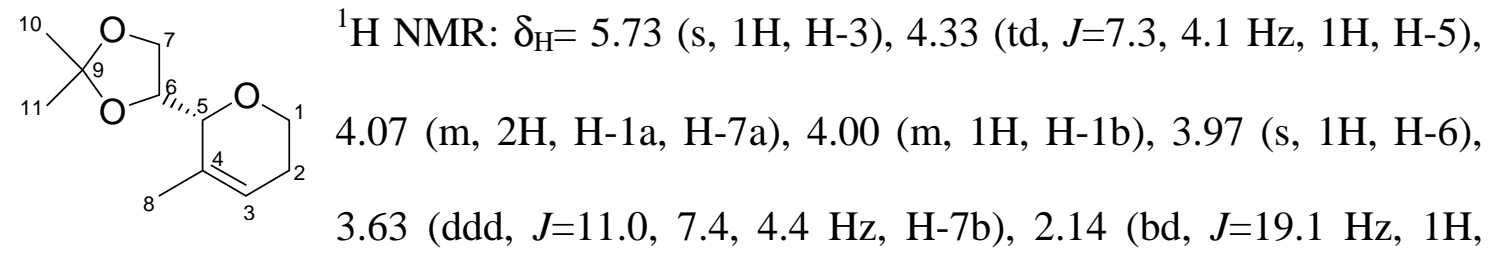
H-2a), 2.00 (bd, J=17.1 Hz, 1H, H-2b), 1.71 (d, J=0.5 Hz, 3H, H-8), 1.45 (s, 3H, H-10), 1.35 (s, 3H, H-11).

${ }^{13} \mathrm{C}$ NMR: $\delta_{\mathrm{C}}=132.2(\mathrm{C}-4), 122.9(\mathrm{C}-3), 109.1(\mathrm{C}-9), 76.2(\mathrm{C}-5), 75.2(\mathrm{C}-6), 65.5$ (C-7), 62.9 (C-1), 26.1 (C-11), 25.4 (C-10), 25.2 (C-2), 19.7 (C-8).

\section{(2S)-(2-methoxycabonyl-E-ethylene)-3-methyl-5,6-dihydro-(2H)-pyran (6.10).}

To a solution of diol $6.7(100 \mathrm{mg}, 0.63 \mathrm{mmol})$ in wet $\mathrm{CH}_{2} \mathrm{Cl}_{2}(2 \mathrm{~mL})$ at ambient temperature was added BAIB (243 $\mathrm{mg}, 0.756 \mathrm{mmol})$ and TEMPO (10 $\mathrm{mg}, 0.063$ 
mmol). The mixture was stirred until no starting material remained by TLC ( $4 \mathrm{~h})$ and then was added 1-methyltriphenylphosphoranylidene-2-propanoate (274mg, $0.82 \mathrm{mmol})$ and the reaction stirred for $12 \mathrm{~h}$. The mixture was loaded onto a silica gel column and eluted with hexanes:EtOAc (5:1) to give $\mathbf{6 . 9}$ as a colourless oil (37 mg, 28\%) along with byproduct $\mathbf{6 . 1 0}$ as a colourless oil (42 $\mathrm{mg}, 31 \%)$.

6.9. ${ }^{1} \mathrm{H}$ NMR: $\delta_{\mathrm{H}}=7.06(\mathrm{dd}, J=15.6,4.1 \mathrm{~Hz}, 1 \mathrm{H}, \mathrm{H}-7), 6.17$ (td, (s, 3H, H-10), 3.56 (td, J=10.8, 3.4 Hz, 1H, H-1b), 2.28 (m, 1H, H-2a), 1.90 (m, 1H, H2b), $1.70(\mathrm{~m}, 3 \mathrm{H}, \mathrm{H}-11)$.

${ }^{13} \mathrm{C}$ NMR: $\delta_{\mathrm{C}}=166.8(\mathrm{C}-9), 148.9$ (C-7), 131.9 (C-4), 123.9 (C-3), $121.1(\mathrm{C}-8), 78.9$ (C5), 70.3 (C-6), 63.6 (C-1), 51.6 (C-10), 25.7 (C-2), 19.0 (C-11).

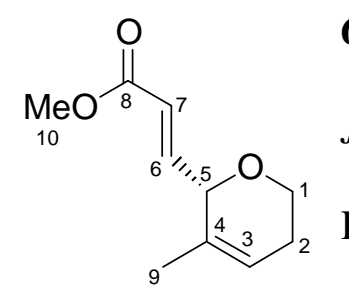

6.10. ${ }^{1} \mathrm{H}$ NMR: $\delta_{\mathrm{H}}=6.95(\mathrm{dd}, J=15.5,5.9 \mathrm{~Hz}, 1 \mathrm{H}, \mathrm{H}-6), 6.06(\mathrm{dd}$, $J=15.7,1.5 \mathrm{~Hz}, 1 \mathrm{H}, \mathrm{H}-7), 5.65(\mathrm{~s}, 1 \mathrm{H}, \mathrm{H}-3), 4.55(\mathrm{~d}, J=3.7 \mathrm{~Hz}, 1 \mathrm{H}$, H-5), 3.82 (dd, J=11.2, 5.5Hz, 1H, H-1a), 3.74 (s, 3H, H-10), 3.70

(dd, $J=11.2,5.7 \mathrm{~Hz}, 1 \mathrm{H}, \mathrm{H}-1 \mathrm{~b}), 2.10$ (m, 2H, H-2), 1.64 (s, 3H, H-9).

${ }^{13} \mathrm{C}$ NMR: $\delta_{\mathrm{C}}=166.8(\mathrm{C}-8), 145.3(\mathrm{C}-6), 132.4(\mathrm{C}-4), 121.9(\mathrm{C}-7), 121.5(\mathrm{C}-3), 75.8(\mathrm{C}-$ 5), $61.4(\mathrm{C}-1), 51.6(\mathrm{C}-10), 25.2(\mathrm{C}-2), 19.6(\mathrm{C}-9)$.

IR: $2953,1721,1658,1435,1267,1169,1048,980,913,731 \mathrm{~cm}^{-1}$.

\section{(2S)-(pen-2-E-ene-1-hydroxy-4-one)-3-methyl-5,6-dihydro-(2H)-pyran (6.11).}

To a solution of $\mathbf{6 . 7}(97 \mathrm{mg}, 0.61 \mathrm{mmol})$ in wet $\mathrm{CH}_{2} \mathrm{Cl}_{2}(10 \mathrm{~mL})$ at ambient temperature was added BAIB (236 mg, $0.73 \mathrm{mmol})$ and TEMPO (10 mg, $0.063 \mathrm{mmol})$. The mixture was stirred until no starting material remained by TLC $(4 \mathrm{~h})$ and then was added 1(triphenylphosphoranylidene)-2-propane $(274 \mathrm{mg}, 0.819 \mathrm{mmol})$ and the reaction stirred 
for $12 \mathrm{~h}$. The resulting mixture was loaded onto a silica gel column and eluted with hexanes:EtOAc (5:1) to give $\mathbf{6 . 1 1}$ as a colourless oil (47 mg, 39\%).

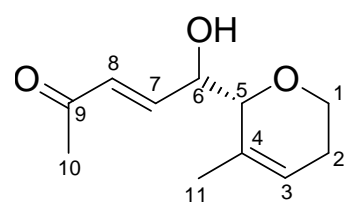

${ }^{1} \mathrm{H}$ NMR: $\delta_{\mathrm{H}}=6.73(\mathrm{dd}, J=16.1,4.6 \mathrm{~Hz}, 1 \mathrm{H}, \mathrm{H}-7), 6.23(\mathrm{dd}$, $J=15.9,1.7 \mathrm{~Hz}, 1 \mathrm{H}, \mathrm{H}-8), 5.63(\mathrm{~d}, J=5.2 \mathrm{~Hz}, 1 \mathrm{H}, \mathrm{H}-3), 4.31$ (m, 1H, H-6), 3.96 (s, 1H, H-5), 3.82 (dd, J=11.0, 5.4Hz, 1H, H-1a), 3.41 (td, J=11.0, 3.7 Hz, 1H, H-1b), 2.25 (bs, 1H, -OH), 2.13 (m, 1H, H-2a), 2.13 (s, 3H, H-10), 1.75 (m, 1H, H-2b), 1.56 (s, 3H, H-11).

${ }^{13}$ C NMR: $\delta_{C}=198.3(\mathrm{C}-9), 147.3$ (C-7), 131.9 (C-4), 130.3 (C-8), 124.0 (C-3), 79.0 (C5), 70.5 (C-6), 63.6 (C-1), 27.5 (C-10), 25.1 (C-2), 19.0 (C-11).

\section{(2S)-(1-acetyl-pen-2-E-ene-1-hydroxy-4-one)-3-methyl-5,6-dihydro-(2H)-pyran} (6.13).

To a solution of $\mathbf{6 . 1 1}(33 \mathrm{mg}, 0.168 \mathrm{mmol})$ in $\mathrm{CH}_{2} \mathrm{Cl}_{2}(1 \mathrm{~mL})$ at ambient temperature was added $\mathrm{Ac}_{2} \mathrm{O}(32 \mu \mathrm{L}, 0.336 \mathrm{mmol})$ and pyridine $(41 \mu \mathrm{L}, 0.504 \mathrm{mmol})$ and the mixture stirred for $12 \mathrm{~h}$. The resulting mixture was poured onto $\mathrm{Et}_{2} \mathrm{O}(10 \mathrm{~mL})$ and washed with $\mathrm{H}_{2} \mathrm{O}\left(2 \mathrm{x}\right.$ 10mL). The organic layer was dried over $\mathrm{MgSO}_{4}$ and concentrated in vacuo. Flash chromatography on silica gel with hexanes: EtOAc $(5: 1)$ gave 6.13 as a colourless oil (27 $\mathrm{mg}, 67 \%)$.

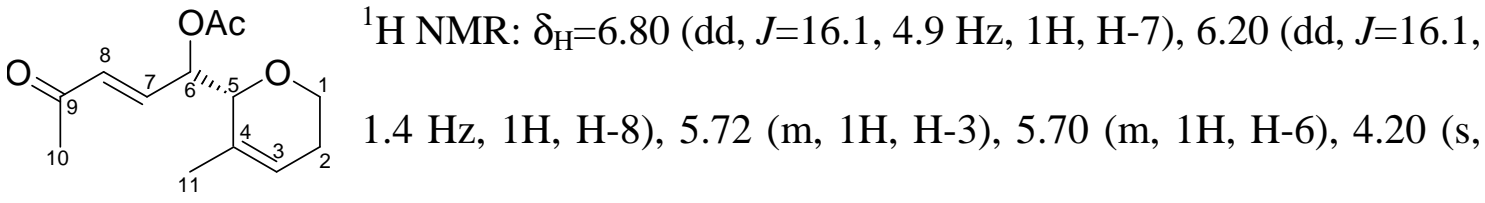
1H, H-5), 3.99 (m, 1H, H-1a), 3.57 (m, 1H, H-1b), 2.27 (s, 3H, H-10), 2.23 (m, 1H, H2a), 2.11 (s, 3H, H-Ac- $\left.\underline{\mathrm{CH}}_{3}\right), 1.94$ (m, 1H, H-2b), 1.61 (s, 3H, H-11).

${ }^{13} \mathrm{C}$ NMR: $\delta_{\mathrm{C}}=197.8\left(\mathrm{C}-\mathrm{Ac}-\underline{\mathrm{CH}}_{3}\right), 169.8(\mathrm{C}-9), 142.2(\mathrm{C}-7), 131.1$ (C-4), $131.0(\mathrm{C}-8)$, 123.8 (C-3), 77.4 (C-5), 72.2 (C-6), 63.4 (C-1), 27.1 (C-10), 25.0 (C-2), 20.8 (C-Ac), $19.0(\mathrm{C}-11)$.

IR: $2924,1743,1678,1473,1372,1228,1114,1047,977,915,731 \mathrm{~cm}^{-1}$. 


\section{(2S)-(4-methyl-2,4-E-dipenten-1-ol)-3-methyl-5,6-dihydro-(2H)-pyran (6.14).}

To a solution of $\mathrm{MePPh}_{3} \mathrm{Br}(105 \mathrm{mg}, 0.295 \mathrm{mmol})$ in $\mathrm{THF}(1 \mathrm{~mL})$ at $0{ }^{\circ} \mathrm{C}$ was added 1.5 $\mathrm{M} \mathrm{BuLi}$ in hexanes $(160 \mu \mathrm{L}, 0.24 \mathrm{mmol})$ and the mixture stirred for $1 \mathrm{~h}$. The mixture was cooled to $-78{ }^{\circ} \mathrm{C}$ and $\mathbf{6 . 1 3}(14 \mathrm{mg}, 0.059 \mathrm{mmol})$ in THF (1mL) added dropwise. The mixture was allowed to warm to ambient temperature and stirred for $3 \mathrm{~h}$. Five drops of water were added to the mixture and stirred for 5 minutes. The reaction mixture was poured onto $\mathrm{Et}_{2} \mathrm{O}(10 \mathrm{~mL})$, washed with saturated aq $\mathrm{NaHCO}_{3}(10 \mathrm{~mL})$ and water $(10 \mathrm{~mL})$, dried over $\mathrm{MgSO}_{4}$ and concentrated in vacuo. Flash chromatography on silica gel with hexanes: EtOAc $(5: 1)$ gave $\mathbf{6 . 1 4}$ as a colourless oil (11 mg, 95\%).

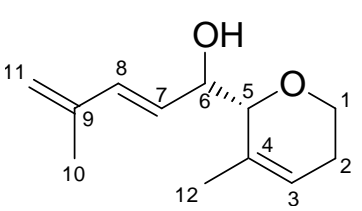

${ }^{1} \mathrm{H}$ NMR: $\delta_{\mathrm{H}}=6.42(\mathrm{dd}, J=15.9,0.8 \mathrm{~Hz}, 1 \mathrm{H}, \mathrm{H}-8), 5.86(\mathrm{dd}$, $J=15.9,6.6 \mathrm{~Hz}, 1 \mathrm{H}, \mathrm{H}-7), 5.76$ (m, 1H, H-3), 5.00, (s, 2H, H-11),

4.34 (m, 1H, H-6), 4.02 (m, 1H, H-1a), 4.01 (m, 1H, H-5), 3.61 (ddd, $J=11.0,10.0,3.9 \mathrm{~Hz}, 1 \mathrm{H}, \mathrm{H}-1 \mathrm{~b}), 2.28$ (m, 1H, H-2a), 2.27 (d, J=7.3 Hz, 1H, -O프), 1.95 (m, 1H, H-2b), 1.88 (t, J=1.0 Hz, 3H, H-12), 1.72 (m, 3H, H-10). IR: $3448,2919,2857,1736,1609,1436,1376,1235,1115,1048,967,910,732 \mathrm{~cm}^{-1}$.

(2S)-(1-phenylxanthate-4-methyl-2,4-dipentene-1-ol)-3-methyl-5,6-dihydro-(2H)pyran (6.15)

To a mixture of $6.14(18 \mathrm{mg}, 0.093 \mathrm{mmol})$ in $\mathrm{CH}_{2} \mathrm{Cl}_{2}(1 \mathrm{~mL})$ at $0{ }^{\circ} \mathrm{C}$ was added DMAP (13mg, $0.93 \mathrm{mmol})$ and phenylchlorothionoformate $(73 \mathrm{mg}, 0.465 \mathrm{mmol})$ and the reaction stirred for $12 \mathrm{~h}$ at ambient temperature. The mixture was poured onto $\mathrm{Et}_{2} \mathrm{O}$ (10mL), washed with $\mathrm{H}_{2} \mathrm{O}(3 \times 10 \mathrm{~mL})$, dried over $\mathrm{MgSO}_{4}$ and concentrated in vacuo. Flash chromatography on silica gel with hexanes: EtOAc (25:1) gave 6.15 as a colourless oil (19 mg, 70\%). 


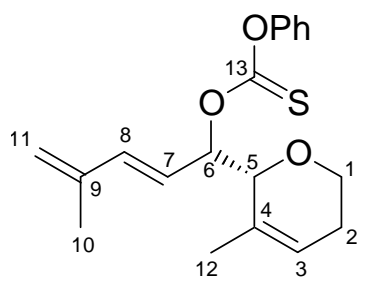

${ }^{1} \mathrm{H}$ NMR: $\delta_{\mathrm{H}}=7.37$ (m, 2H, H-Ar), 7.23 (m, 1H, H-Ar), 7.14, (m, 2H, H-Ar), 5.87 (dd, $J=14.6,6.9 \mathrm{~Hz}, 1 \mathrm{H}, \mathrm{H}-8), 5.79$ (dd, $J=15.4$, $6.9 \mathrm{~Hz}, 1 \mathrm{H}, \mathrm{H}-7), 5.62$ (m, 1H, H-6), 5.09 (d, J=0.7 Hz, 1H, H11), 4.98 (m, 1H, H-11), 4.60 (d, J=7.3 Hz, 1H, H-6), 4.42 (d, J=7.1 Hz, 1H, H-5), 3.85 (m, 1H, H-1a), 3.65 (m, 1H, H-1b), 2.10 (m, 2H, H-2), 1.87 (m, 3H, H-10), 1.58 (s 3H, $\mathrm{H}-12)$.

\section{(2S)-((1S)-1,2-dihydroxy)ethyl)-3-methyl-5-hydro-6-deuetro-(2H)-pyran.}

To a mixture of $6.7(42 \mathrm{mg}, 0.13 \mathrm{mmol})$ and TESD (106 $\mu \mathrm{L}, 0.6 \mathrm{mmol})$ in $\mathrm{MeCN}$ $(1 \mathrm{~mL})$ at $0{ }^{\circ} \mathrm{C}$ was added dropwise TMSOTf $(12 \mu \mathrm{L}, 0.06 \mathrm{mmol})$. The mixture was allowed to warm to ambient temperature and stirred for $4 \mathrm{~h}$. The reaction was quenched with $\mathrm{Et}_{3} \mathrm{~N}(100 \mu \mathrm{L}, 0.72 \mathrm{mmol})$ and poured onto a silica plug. The silica plug was washed with hexanes $(150 \mathrm{~mL})$ to remove excess TESD, and then washed with EtOAc $(2 \times 50 \mathrm{~mL})$. The EtOAc washings were concentrated in vacuo to give a mixture of 6.17 and 6.18 as a colourless oil (10 $\mathrm{mg}, 48 \%)$ in a 6:1 ratio by GC.

Major isomer 6.17. ${ }^{1} \mathrm{H}$ NMR: $\delta_{\mathrm{H}}=5.76(\mathrm{~d}, J=4.1 \mathrm{~Hz}, 1 \mathrm{H}, \mathrm{H}-3), 4.14$
${ }_{3}$ $J=8.6 \mathrm{~Hz}, 1 \mathrm{H},-\mathrm{OH}), 2.29$ (m, 1H, H-2a), 1.90, (m, 1H, H-2b), 1.71 (s, 3H, H-8).

${ }^{13} \mathrm{C}$ NMR: $\delta_{\mathrm{C}}=132.6(\mathrm{C}-4), 122.8(\mathrm{C}-3), 79.1(\mathrm{C}-5), 70.4(\mathrm{C}-6), 65.5(\mathrm{C}-7), 63.3(\mathrm{t}$, $J=21.6 \mathrm{~Hz}, \mathrm{C}-1), 25.1$ (C-2), $19.2(\mathrm{C}-8)$. 


\section{Appendix A}

To confirm that the reactions lead to the postulated oxepine products, an example was assigned from first principles. The example chosen was $(4 \mathrm{a} R, 9 \mathrm{a} R)$-6-Azido-2,2-di-tertbutyl-4a,6,7,9a-tetrahydro-4H-1,3,5-trioxa-2-silabenzocycloheptene $(\mathbf{3 . 5 a} \& \mathbf{3 . 5 b})$ as shown in Figure A.1.

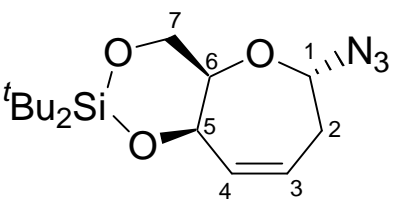

$3.5 \mathrm{a}$

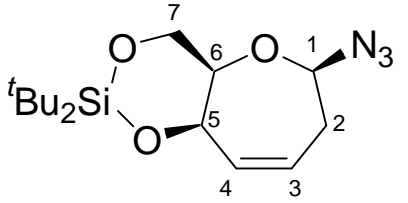

$3.5 b$

Figure A.1. Major 3.5a and minor 3.5b oxepines showing NMR numbering scheme.

This example was chosen as both isomers were separable and thus the stereochemistry of the major and minor isomer could be individually established. This information could then be extrapolated to the other products. Unfortunately with this example it was not possible to get any high resolution mass spectrometry data. However, all 15 carbons, 27 protons, three nitrogens and one silicon could be accounted for. Presence of an azide group was suggested by IR, which showed a strong peak at $2103 \mathrm{~cm}^{-1}$ in the major epimer 3.5a and $2109 \mathrm{~cm}^{-1}$ in the minor epimer $\mathbf{3 . 5 b}$.

The minor component 3.5b was assigned first as there were no overlapping signals in the ${ }^{1} \mathrm{H}$ NMR spectrum, and a more complete NMR experiment set was available including NOE and HMBC data. The presence of a di-tert-butylsilyl protecting group was indicated by ${ }^{1} \mathrm{H}$ and ${ }^{13} \mathrm{C}$ NMR resonances for the tert-butyl methyl groups $\left(\delta_{\mathrm{H}} 1.06\right.$, $\delta_{\mathrm{C}} 27.5$ and $\left.\delta_{\mathrm{H}} 1.08, \delta_{\mathrm{C}} 27.4\right)$ and the quaternary carbons $\left(\delta_{\mathrm{C}} 23.1\right.$ and $\left.\delta_{\mathrm{C}} 21.1\right)$. HMBC correlations were observed between the tert-butyl methyl groups and also to the tertbutyl quaternary carbons. Integration of the ${ }^{1} \mathrm{H}$ resonances gave approximately 18 protons. ${ }^{29} \mathrm{Si}$ NMR gave one peak at $\delta_{\mathrm{Si}}-8.74$. HSQC-DEPT revealed the presence of 
three oxymethines, two alkenic methines, an oxymethylene group and a methylene. The COSY spectrum revealed the presence of a single ${ }^{1} \mathrm{H}$ spin system. The connectivity of the ${ }^{1} \mathrm{H}$ spin system was then constructed from a series of COSY correlations from the $\mathrm{C}-1$ azamethine $\left(\delta_{\mathrm{H}} 4.82, \delta_{\mathrm{C}} 90.6\right)$ to a methylene $\left(\mathrm{C}-2: \delta_{\mathrm{H}} 2.67\right.$, $\left.\delta_{\mathrm{C}} 36.0\right)$ to an alkenic methine $\left(\mathrm{C}-3: \delta_{\mathrm{H}} 5.75, \delta_{\mathrm{C}} 122.4\right)$ to a second alkenic methine $\left(\mathrm{C}-4 ; \delta_{\mathrm{H}} 5.94, \delta_{\mathrm{C}} 133.9\right)$ to an oxymethine $\left(\mathrm{C}-5: \delta_{\mathrm{H}} 4.74, \delta_{\mathrm{C}} 72.9\right)$ to a second oxymethine (C-6: $\left.\delta_{\mathrm{H}} 3.70, \delta_{\mathrm{C}} 77.4\right)$ to an oxymethylene $\left(\mathrm{C}-7: \delta_{\mathrm{H}} 4.26, \delta_{\mathrm{C}} 68.3\right)$. This spin system is illustrated in Figure A.2.

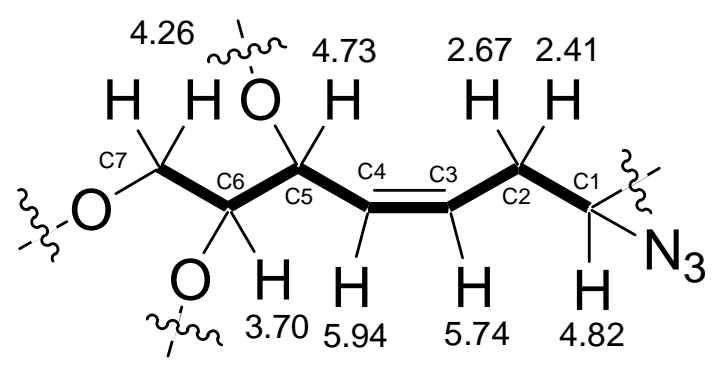

Figure A.2. COSY assignment of the C7 backbone of the minor isomer 3.5b.

An HMBC correlation from the proton resonances of $\mathrm{C} 1$ to $\mathrm{C} 6$ clearly established the presence of the seven membered ring by established an oxygen bridge between these two centres. Finally to establish the stereochemistry NOESY data gave clear correlations between $\mathrm{H}-1$ and H-6, conclusively assigning the minor isomer as the $\beta$ substituted azido oxepine. The relative stereochemistry of C-5 and C-6 was taken to be the same as in the starting galactal and was assigned as such. Finally the presence of the azide group was confirmed by ${ }^{15} \mathrm{~N}-{ }^{1} \mathrm{H}$ HMBC measurements. This gave 3 signals at $\delta_{\mathrm{N}}-135.8,-288.4$ and -300.1 . The nitrogen at $\delta_{\mathrm{N}}-288.4$ had correlations from the protons at C-1, C-2 and C-3. The nitrogen at $\delta_{\mathrm{N}}-135.8$ showed correlations from the 
protons of $\mathrm{C}-1$ and $\mathrm{C}-2$. The third nitrogen at $\delta_{\mathrm{N}}-300.1$ showed a single correlation from the proton at C-1. These could thus be assigned as N-1, N-2 and N-3 respectively.

Moving to the major isomer, again no HRMS data could be obtained for the compound. However all 15 carbon and 27 protons could be accounted for. ${ }^{13} \mathrm{C}$ and ${ }^{1} \mathrm{H}$ resonances consistent with a di-tert-butylsilyl protecting group were found and assigned as before. HSQC-DEPT revealed the presence of three oxymethines, two alkenic methines, an oxymethylene and a methylene. COSY correlations again revealed the presence of one ${ }^{1} \mathrm{H}$ spin system. The connectivity of the ${ }^{1} \mathrm{H}$ spin system was then constructed from a series of COSY correlations from the C-1 azamethine $\left(\delta_{\mathrm{H}} 5.42, \delta_{\mathrm{C}} 90.1\right)$ to a methylene $\left(\mathrm{C}-2 ; \delta_{\mathrm{H}} 2.48\right.$ and $\left.\delta_{\mathrm{H}} 2.30, \delta_{\mathrm{C}} 31.0\right)$ to an alkenic methine $\left(\mathrm{C}-3: \delta_{\mathrm{H}} 5.61, \delta_{\mathrm{C}} 122.4\right)$ to a second alkenic methine $\left(\mathrm{C}-4: \delta_{\mathrm{H}} 5.71, \delta_{\mathrm{C}} 133.9\right)$ to an oxymethine $\left(\mathrm{C}-5: \delta_{\mathrm{H}} 4.86, \delta_{\mathrm{C}}\right.$ $74.9)$ to a second oxymethine $\left(\mathrm{C}-6: \delta_{\mathrm{H}} 4.13, \delta_{\mathrm{C}} 70.0\right)$ to an oxymethylene $\left(\mathrm{C}-7: \delta_{\mathrm{H}} 4.32\right.$, $\left.\delta_{\mathrm{C}} 68.1\right)$ as shown in Figure A.3.

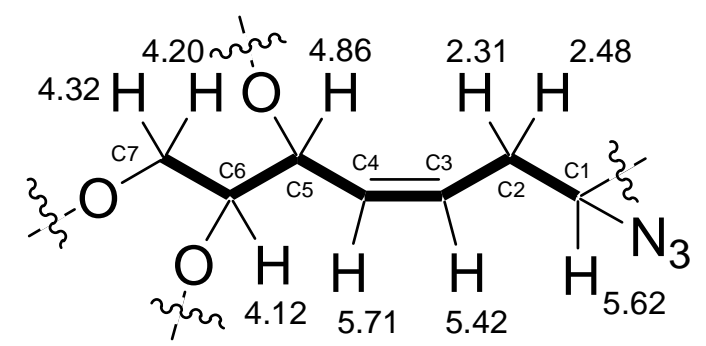

Figure A.3. COSY assignment of the C7 backbone of the major isomer 3.5a.

Unfortunately no HMBC data was available for this compound and so the ring linkage could not be positively determined between C-1 and C- 6 . As the link has been assigned in the minor epimer it was extrapolated to be present in the major epimer. 


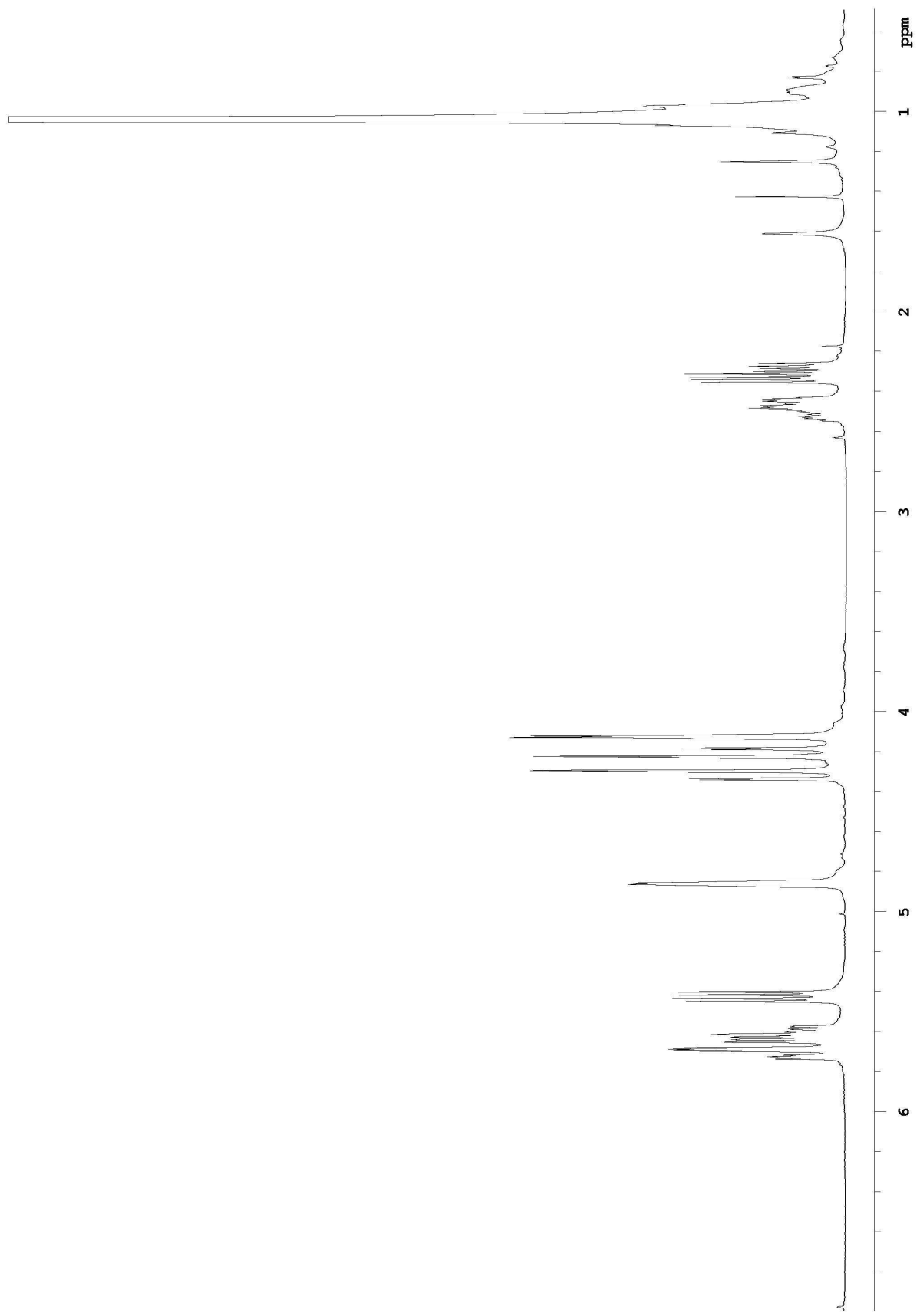

${ }^{1} \mathrm{H}$ NMR spectrum of azide 3.5a $\mathrm{In} \mathrm{CDCl}_{3}(500 \mathrm{MHz})$. 


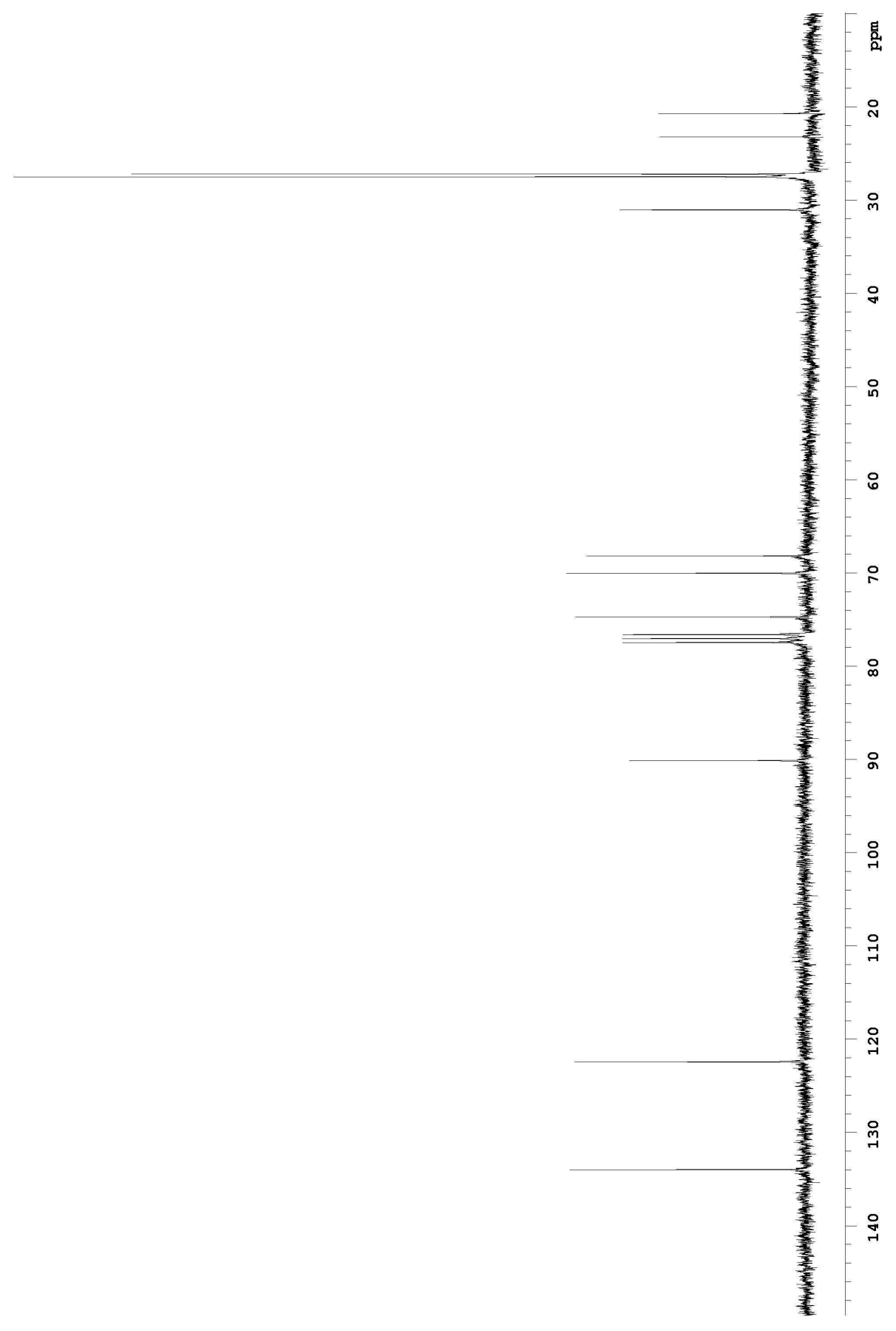

${ }^{13} \mathrm{C}$ NMR spectrum of azide 3.5a In $\mathrm{CDCl}_{3}(500 \mathrm{MHz})$. 


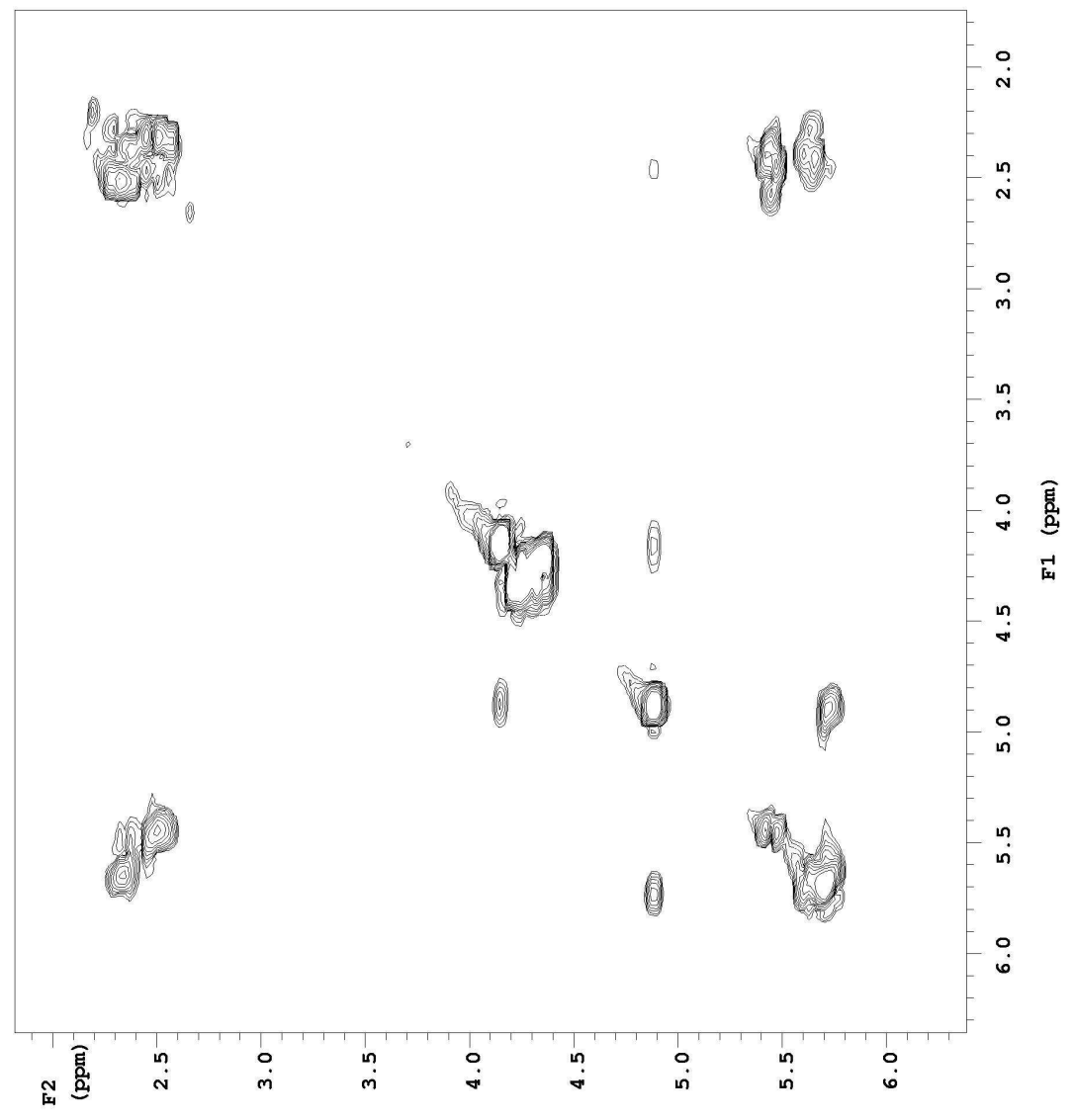

COSY NMR spectrum of azide 3.5a In $\mathrm{CDCl}_{3}(500 \mathrm{MHz})$. 


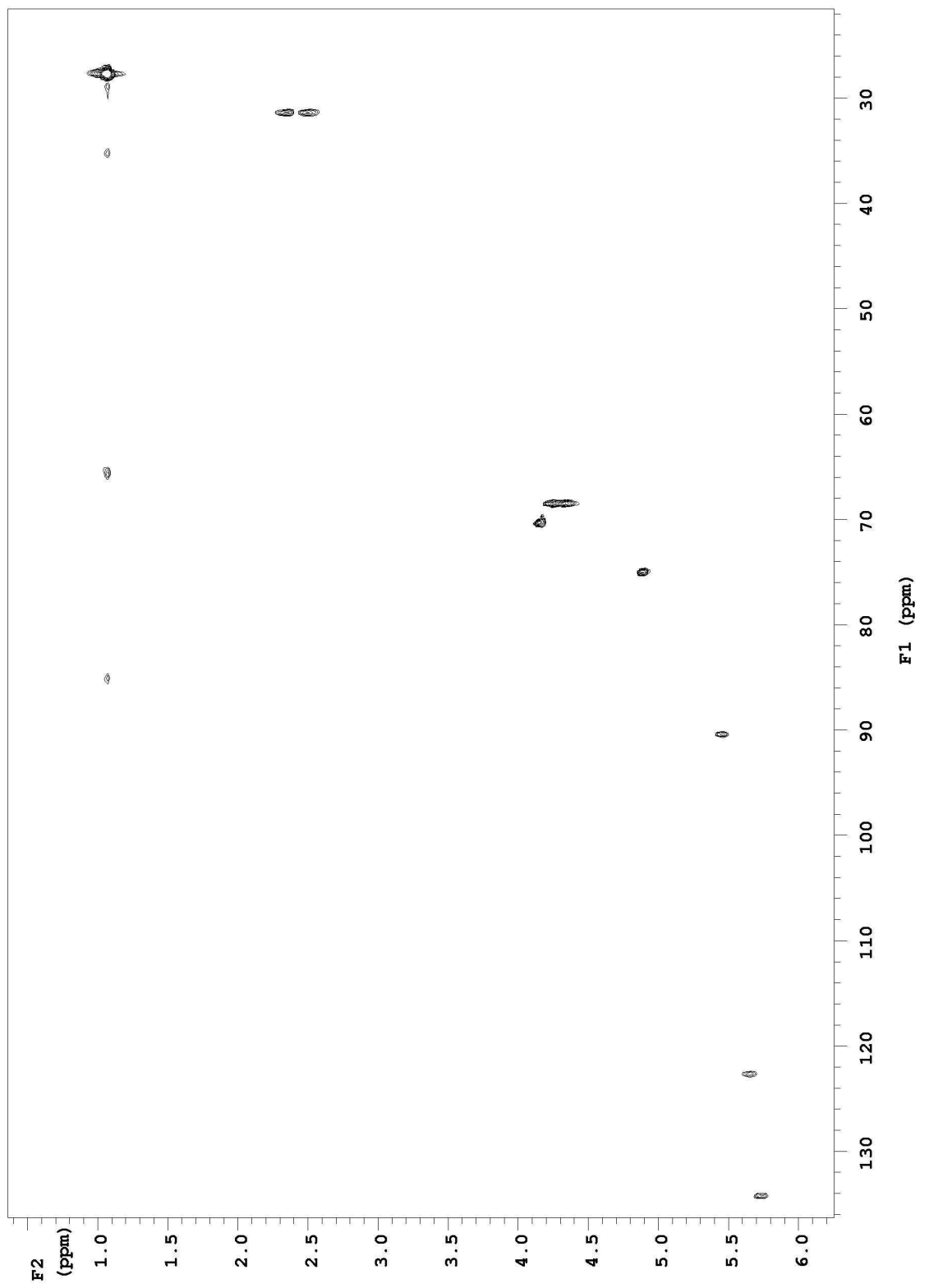

HSQC NMR spectrum of azide 3.5a In $\mathrm{CDCl}_{3}(500 \mathrm{MHz})$. 


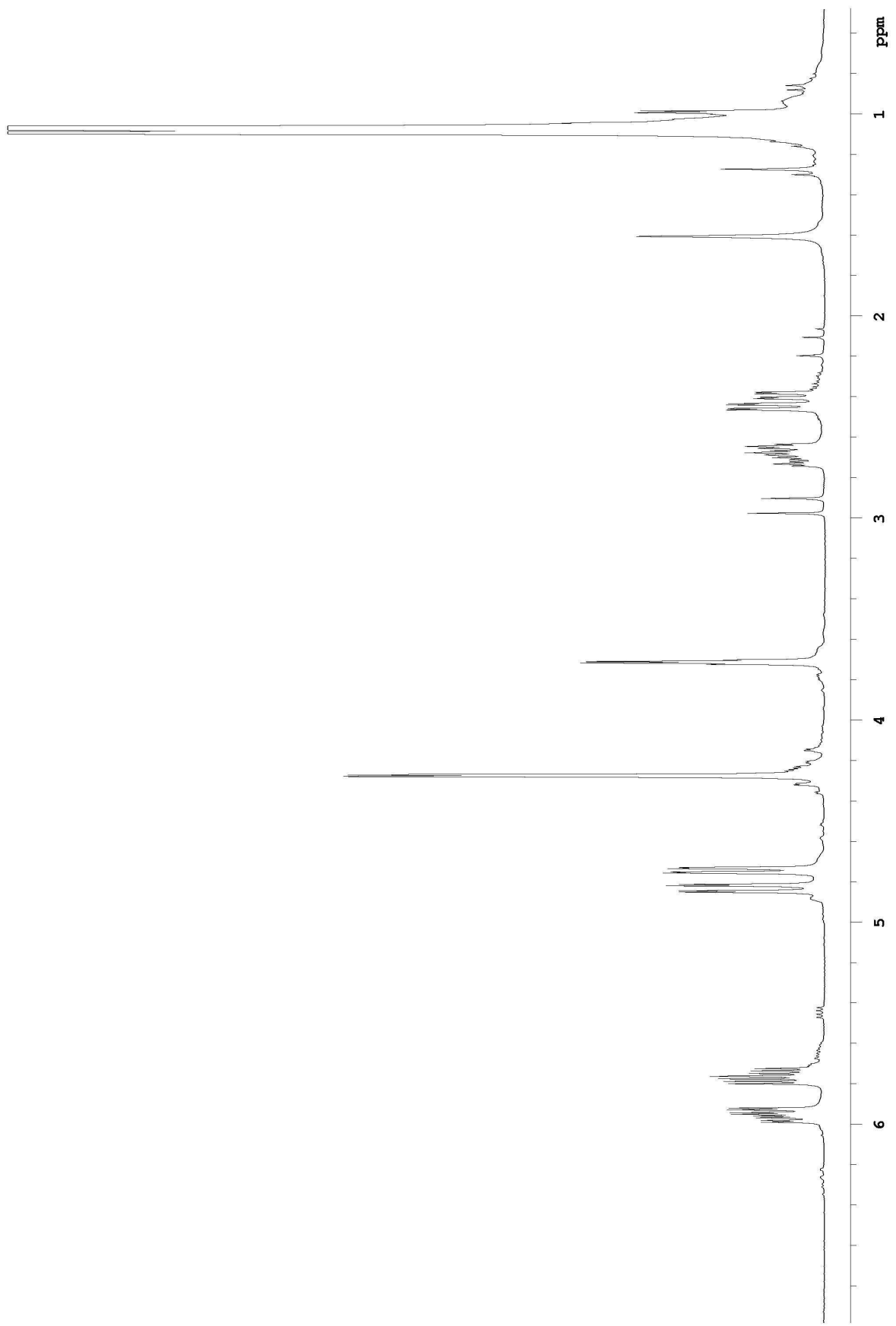

${ }^{1} \mathrm{H}$ NMR spectrum of azide 3.5b $\mathrm{In} \mathrm{CDCl}_{3}(500 \mathrm{MHz})$. 


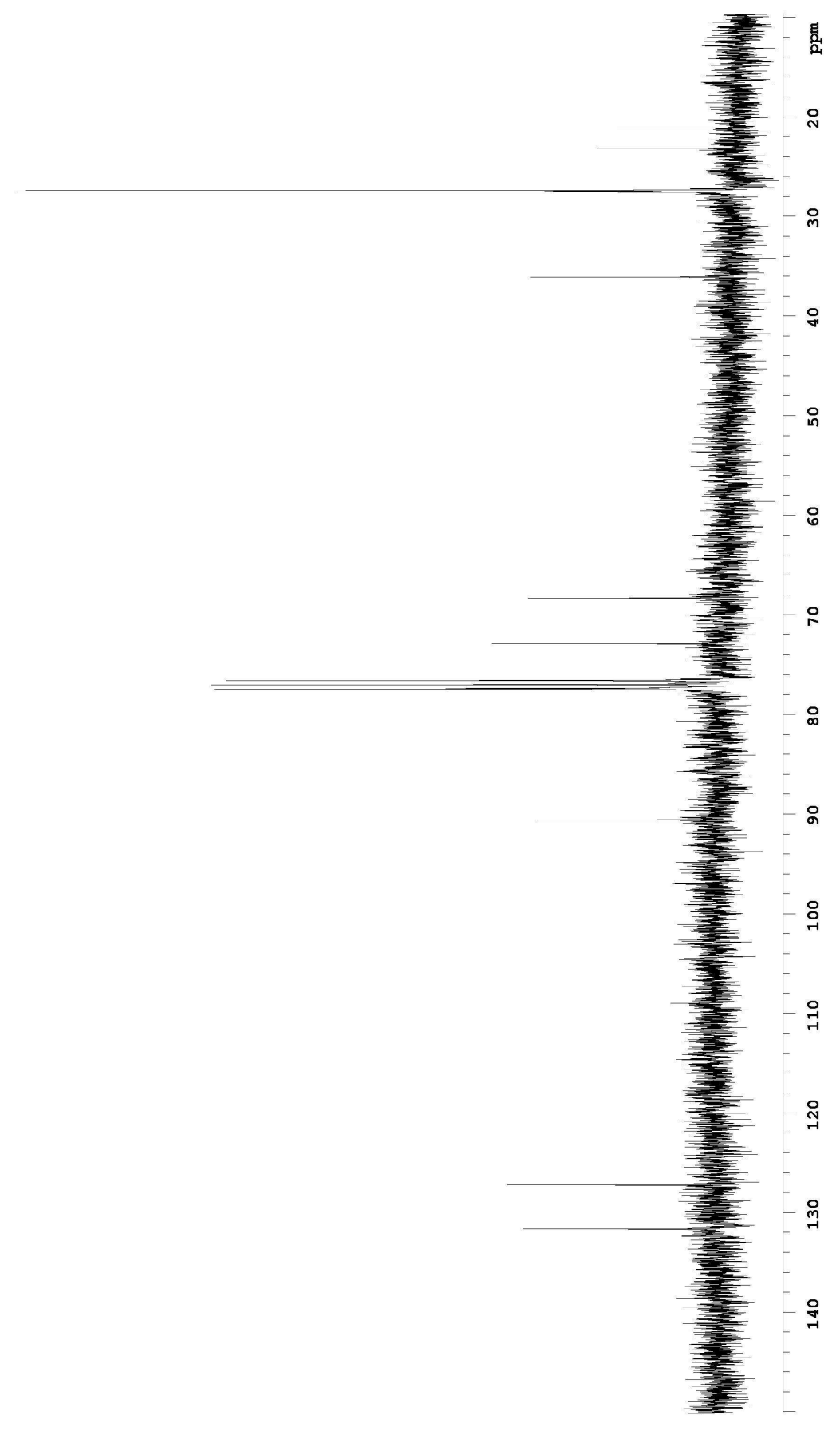

${ }^{13} \mathrm{C}$ NMR spectrum of azide $\mathbf{3 . 5} \mathbf{b}$ In $\mathrm{CDCl}_{3}(500 \mathrm{MHz})$. 


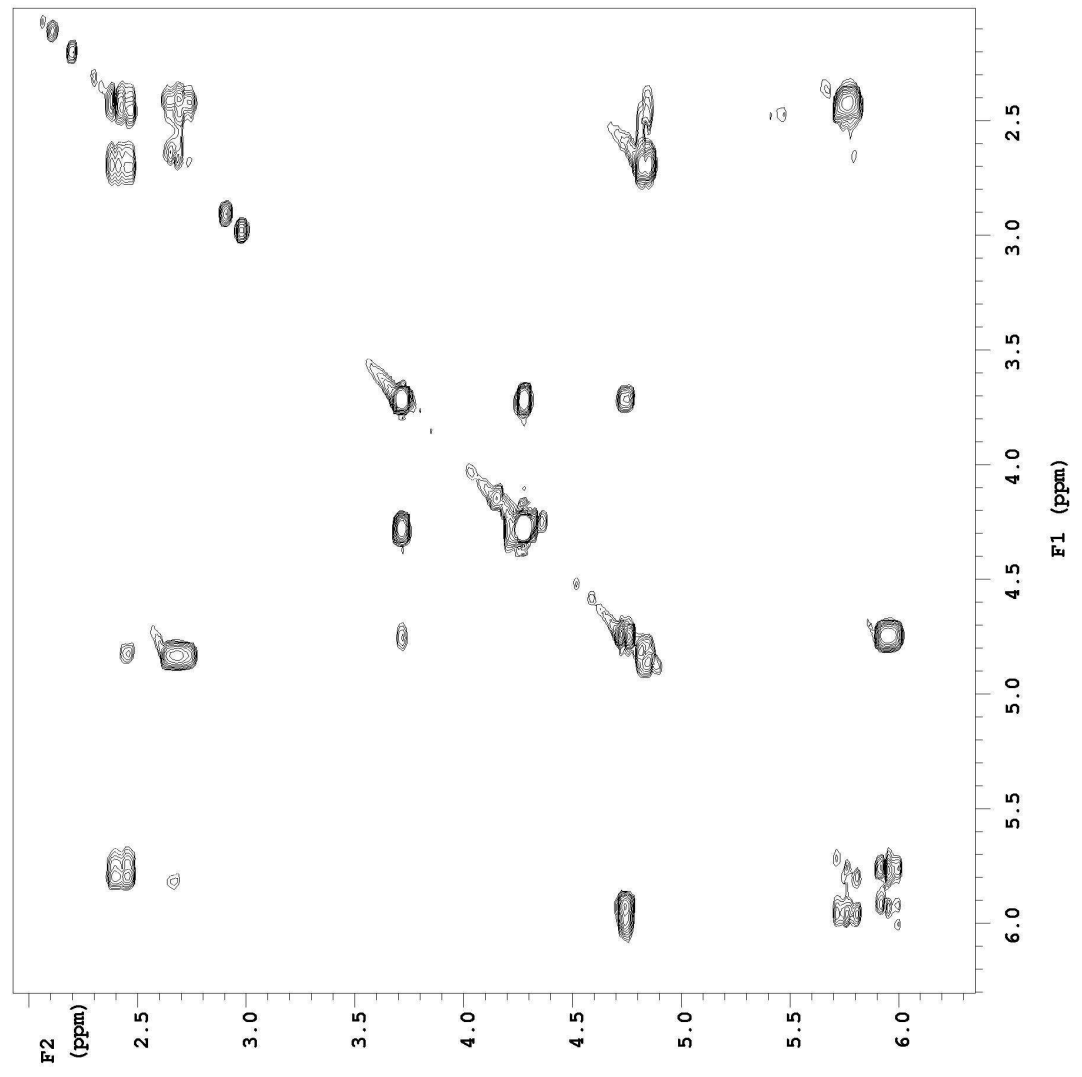

COSY NMR spectrum of azide $\mathbf{3 . 5 b}$ in $\mathrm{CDCl}_{3}(500 \mathrm{MHz})$ 


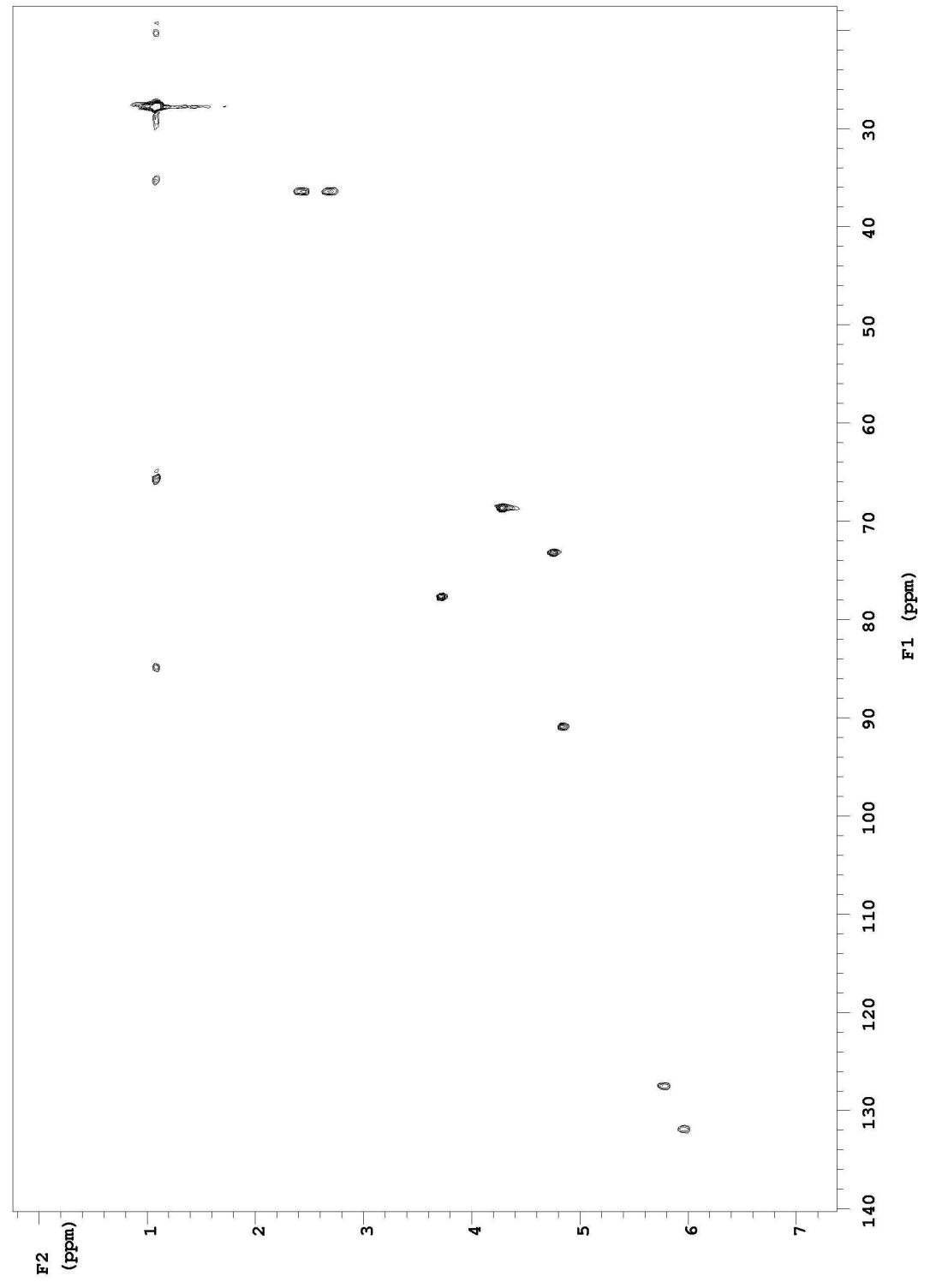

HSQC NMR spectrum of azide 3.5b In $\mathrm{CDCl}_{3}(500 \mathrm{MHz})$. 


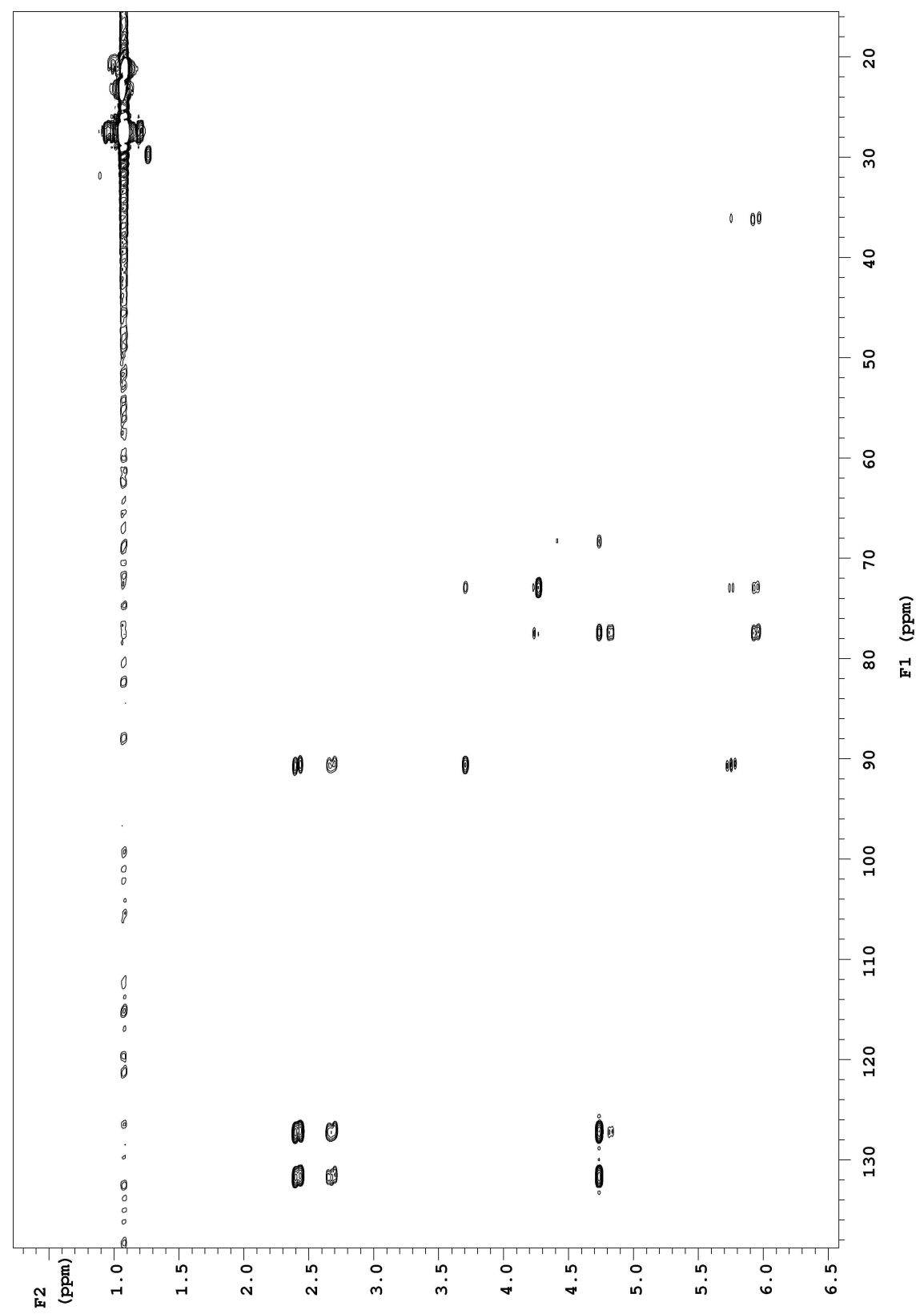

HMBC NMR spectrum of azide $\mathbf{3 . 5 b}$ In $\mathrm{CDCl}_{3}(500 \mathrm{MHz})$. 


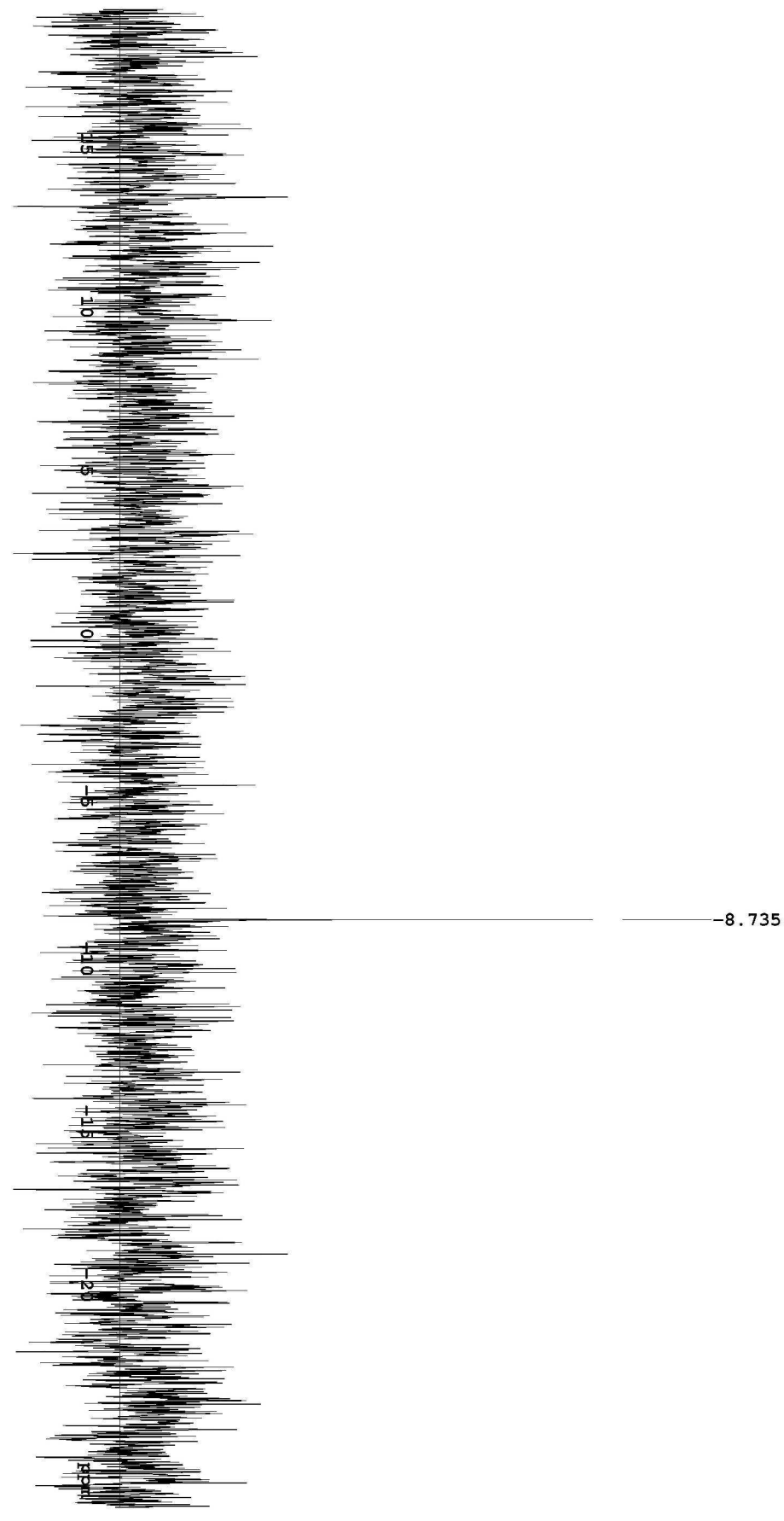

${ }^{29} \mathrm{Si}$ NMR spectrum of azide $\mathbf{3 . 5 b}$ In $\mathrm{CDCl}_{3}(500 \mathrm{MHz})$. 


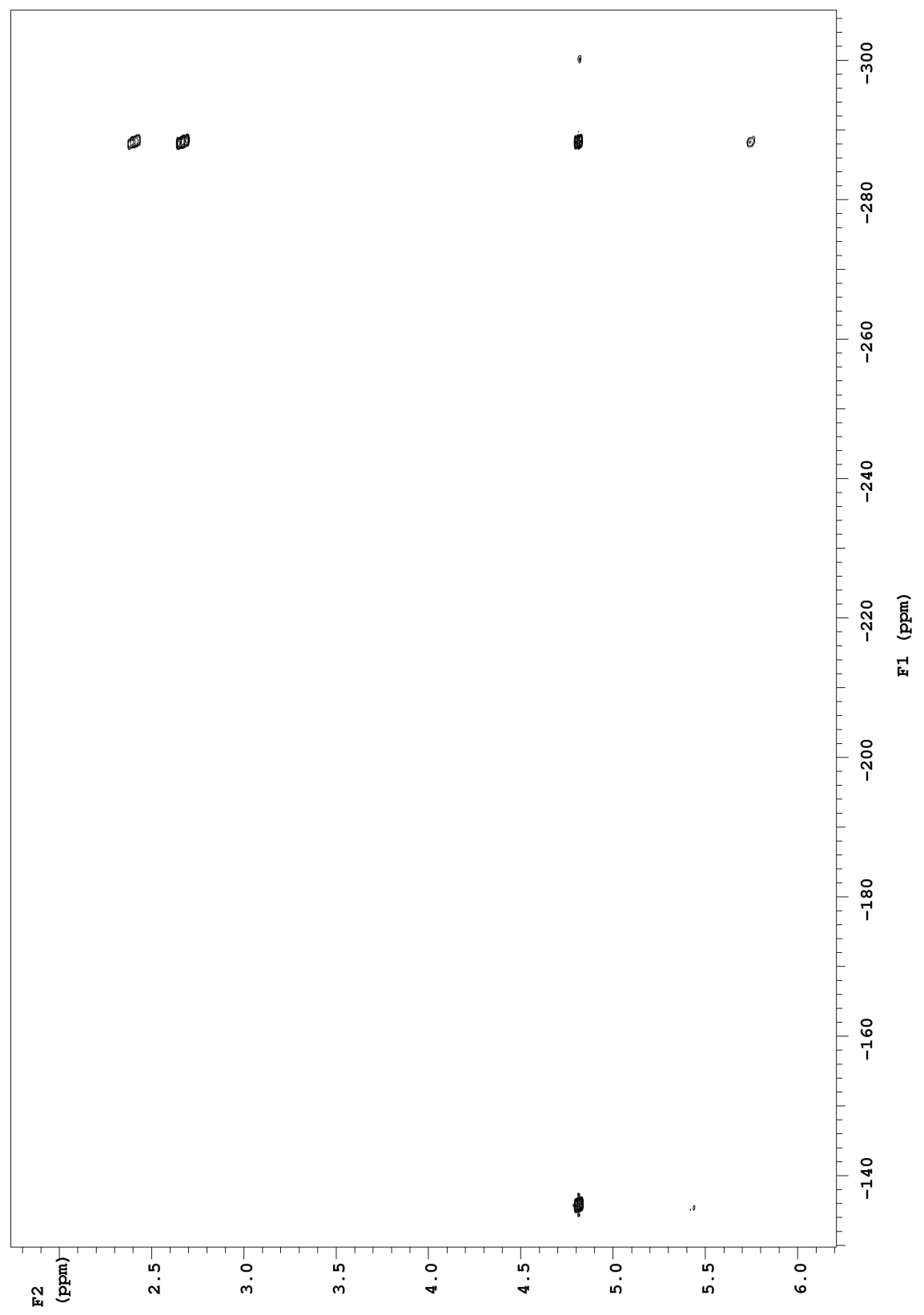

${ }^{15} \mathrm{~N}$ HMBC NMR spectrum of azide 3.5b In $\mathrm{CDCl}_{3}(600 \mathrm{MHz})$. 


\section{Bibliography}

1. Fraser-Reid, B. Acc. Chem. Res. 1975, 8, 192-201.

2. Calvin, M. J. Chem. Soc. 1956, 1895-1914.

3. Calvin, M. http://nobelprize.org/nobel_prizes/chemistry/laureates/1961/calvinlecture.pdf 1961.

4. Hollingsworth, R. I. and Wang, G. Chem. Rev. 2000, 100, 4267-4282.

5. Marshall, J. New Scientist 2007, 28-31.

6. Lichtenthaler, F. W. Eur. J. Org. Chem. 2002, 4095-4122.

7. Lichtenthaler, F. W. Angew. Chem. Int. Ed. Engl. 1992, 31, 1541-1556.

8. Fischer, H. E. http://nobelprize.org/nobel_prizes/chemistry/laureates/1902/ fischer-lecture.pdf.

9. Emil Fisher collection, Bancroft library, UCLA Berkley.

10. Lemieux, R. U.; Kullnig, R. K.; Bernstein, H. J. and Scheneider, W. G. J. Am. Chem. Soc. 1958, 80, 6098-6105.

11. Rademacher, T. W.; Parekh, R. B. and Dwek, R. A. Ann. Rev. Biochem. 1988, $57,785-838$.

12. Lemieux, R. U. Chem. Soc. Rev. 1989, 18, 347-374.

13. Jones, M. J. Organic Chemistry, second edition 2000, W. W. Norton \& Company.

14. Rosanoff, M. A. J. Am. Chem. Soc. 1906, 28, 525-533.

15. Rosanoff, M. A. J. Am. Chem. Soc. 1906, 28, 114-121.

16. Bremer, E. G.; Levery, S. B.; Sonnino, S.; Ghidoni, R.; Canevari, S.; Kannagi, R. and Hakomori, S.-I. J. Biol. Chem. 1984, 259, 14773-14777.

17. Schmidt, R. R. Angew. Chem. Int. Ed. Engl. 1986, 25, 212-235.

18. Ferrier, R. J. J. Chem. Soc. (C) 1964, 5443-5449.

19. Ferrier, R. J. and Prasad, N. Chem. Comm. 1968, 476-477.

20. Ferrier, R. J. and Prasad, N. J. Chem. Soc. (C) 1969, 581-586. 
21. Ferrier, R. J. and Prasad, N. J. Chem. Soc. (C) 1969, 570-575.

22. Danishefsky, S. and Kerwin, J. F. J. Org. Chem. 1982, 47, 3803-3805.

23. Hanna, I. and Wlodyka, P. J. Org. Chem. 1997, 62, 6985-6990.

24. Das, K.; Reddy, A.; Abbineni, C.; Roy, J.; Rao, N.; Sachwani, R. and Iqbal, J.

Tetrahedron Lett. 2003, 44, 4507-4509.

25. Hoberg, J. O. Carbohydr. Res. 1997, 365-367.

26. Hanessian, S. The Total Synthesis of Natural Products. The Chiron Approach., Pergamon Press, New York, 1983.

27. Nicolaou, K. C.; Seitz, S. P. and Pavia, M. R. J. Am. Chem. Soc. 1981, 103, 1222-1224.

28. Nicolaou, K. C.; Pavia, M. R. and Seitz, S. P. J. Am. Chem. Soc. 1981, 103, $1224-1226$.

29. Nicolaou, K. C.; Daines, R. A.; Chakraborty, T. K. and Ogawa, Y. J. Am. Chem. Soc. 1987, 109, 2821-2822.

30. Nicolaou, K. C.; Ajito, K.; Patron, A. P.; Khatuya, H.; Richter, P. K. and Bertinato, P. J. Am. Chem. Soc. 1996, 118, 3059-3060.

31. Nicolaou, K. C.; Hwang, C.-K.; Duggan, M. E.; Nugiel, D. A.; Abe, Y.; Bal Reddy, K.; Defrees, S. A.; Reddy, D. R.; Awartani, R. A.; Conley, S. R.; Rutjes, F. P. J. T. and Theodorakis, E. A. J. Am. Chem. Soc. 1995, 117, 10227-10238.

32. Nicolaou, K. C.; Theodorakis, E. A.; Rutjes, F. P. J. T.; Sato, M.; Tiebes, J.; Xaio, X.-Y.; Hwang, C.-K.; Duggan, M. E.; Yang, Z.; Couladouros, E. A.; Sato, F.; Shin, J.; He, H.-M. and Bleckman, T. J. Am. Chem. Soc. 1995, 117, 1023910251.

33. Nicolaou, K. C.; Rutjes, F. P. J. T.; Theodorakis, E. A.; Tiebes, J.; Sato, M. and Untersteller, E. J. Am. Chem. Soc. 1995, 117, 10252-10263. 
34. Boon, G.-J. and Hale, K. Organic synthesis with carbohydrates 2000, Blackwell publishing, Sheffield.

35. Simmons, H. E. and Smith, R. D. J. Am. Chem. Soc. 1959, 81, 4256-4264.

36. Friedrich, E. C.; Winstein, S. and Poulter, C. D. J. Am. Chem. Soc. 1969, 91, 6892-6894.

37. Nagarajan, M.; Ramana, C. and Murali, R. J. Chem. Soc., Chem Commun. 1995, 217-218.

38. Friedrich, E. C. and Lewis, E. J. J. Org. Chem. 1990, 55, 2491-2494.

39. Furukawa, J.; Kawabata, N. and Nisimura, J. Tetrahedron 1968, 24, 53.

40. Hoberg, J. O. and Bozell, J. Tetrahedron Lett. 1995, 36, 6831-6834.

41. Lorica, R. G.; Albano-Garcia, E. L. and Akkolario, E. M. Nat. Appl. Sci. Bull. 1979, 31, 61-74.

42. Danishefsky, S.; Meng, D.; Bertinato, P.; Balog, A.; Su, D.-S.; Kamenecka, T. and Sorensen, E. J. J. Am. Chem. Soc. 1997, 119, 10073-10092.

43. Boeckman, R. K.; Charette, A. B.; Asberom, T. and Johnston, B. H. J. Am. Chem. Soc. 1987, 109, 7553-7555.

44. Fraser-Reid, B. and Henry, K. Tetrahedron Lett. 1995, 36, 8901-8904.

45. Hoberg, J. O. and Claferty, D. Tetrahedron Lett. 1996, 37, 2533-2536.

46. Brimacombe, J. S.; Evans, M. E.; Forbes, E. J.; Foster, A. B. and Webber, J. M. Carb. Res. 1967, 4, 239.

47. Nagarajan, M.; Ramana, C. V. and Murali, R. J. Org. Chem. 1997, 62, 76947703.

48. Madsen, R.; Beyer, J. and Skaanderup, P. J. Am. Chem. Soc. 2000, 122, 95759583.

49. Hoberg, J. O. and Cousins, G. S. Chem. Soc. Rev. 2000, 29, 165-174. 
50. Sugita, Y.; Kimura, C.; Hosoya, H.; Yamadori, S. and Yokoe, I. Tetrahedron Lett. 2001, 42, 1095-1098.

51. Pietra, F.; Chasera, G.; Mancini, I. and Guella, G. Helv. Chim. Acta. 1992, 75, $310-322$

52. Masamune, T. and Fukuzawa, A. Tetrahedron Lett. 1981, 41, 4081-4084.

53. Proksch, P.; Endrada, R. A.; Wray, V.; Witte, L. and van Ofwegen, L. J. Nat. Prod. 1998, 61, 358-361.

54. Sprogoe, K.; Manniche, S.; Larsen, T. O. and Christophersen, C. Tetrahedron 2005, 61, 8718.

55. Belofsky, G. N.; Anguera, M.; Jensen, P. R.; Fenical, W. and Kock, M. Chem. Eur. J. 2000, 6, 1355-1360.

56. Kashman, Y.; Kakisawa, H.; Hirsh, S.; McConnell, O. J.; Ohlani, I. and Kusami, T. J. Am. Chem. Soc. 1989, 111, 8925-8926.

57. Lin, Y.-Y.; Risk, M.; Ray, S. M.; Van Engen, D.; Clardy, J.; Golik, J.; James, J. C. and Nakanishi, K. J. Am. Chem. Soc. 1981, 103, 6773.

58. Yasumoto, T.; Mutara, M. and Satake, M. J. Am. Chem. Soc. 1993, 115, 361362.

59. Scheuer, P. J.; takahashi, W.; Tsusumi, J. and Yoshida, T. Science 1967, 155, $1267-1268$

60. Peczuh, M. W.; Snyder, N. L. and Haines, H. M. Tetrahedron 2006, 62, 93019320.

61. Suzuki, T.; Matsumura, R.; Oku, K.-I.; Taguchi, K.; Hagiwara, H.; Hoshi, T. and Ando, M. Tetrahedron Lett. 2001, 42, 65-67.

62. Suzuki, T.; Matsumura, R.; Hagiwara, H.; Hoshi, T. and Ando, M. Tetrahedron Lett. 2001, 42, 1543-1546. 
63. Nicolaou, K. C.; Postema, M. H. D. and Claiborne, C. F. J. Am. Chem. Soc. 1996, $118,1565-1566$.

64. Tebbe, F.; Parshall, G. and Reddy, G. J. Am. Chem. Soc. 1978, 100, 3611-3613.

65. Yamamoto, Y. and Kadota, I. J. Org. Chem. 1998, 63, 6597-6606.

66. Rainier, J. D.; Allwein, S. P. and Cox, J. M. J. Org. Chem. 2001, 66, 1380-1386.

67. Jenkins, P. R.; Ghost, S.; Holt, D. J.; Barker, W. D. and Panda, J. J. Org. Chem. 2000, 65, 482-493.

68. Clark, J. S.; Elustondo, F.; Trevitt, G. P.; Boyall, D.; Robertson, J.; Blake, A. J.; Wilson, C. and Stammen, B. Tetrahedron 2002, 58, 1973-1982.

69. Overman, L. E.; Berger, D. and Renhowe, P. A. J. Am. Chem. Soc. 1993, 115, 9305-9306.

70. Hoberg, J. O. J. Org. Chem.. 1997, 62, 6615-6618.

71. Rucker, C. Chem. Rev. 1995, 95, 1009-1064.

72. Bertozzi, C. R.; Winans, K. A.; King, D. S. and Rao, V. R. Biochemistry 1999, $38,11700-11710$.

73. Balasubramanian, K. and Ramesh, N. Tetrahedron 1995, 51, 255-272.

74. Mitsunobu, O. Y. Bull. Chem. Soc. Jpn. 1967, 40, 2380-2382.

75. Hughes, D. L. Org. Prep. 1996, 28, 127-164.

76. Hughes, D. L.; Reamer, R. A.; Bergan, J. J. and Grabowski, E. J. J. J. Am. Chem. Soc. 1988, 110, 6487-6491.

77. Camp, D. and Jenkins, I. D. J. Org. Chem. 1989, 54, 3049-3054.

78. Camp, D. and Jenkins, I. D. J. Org. Chem. 1989, 54, 3045-3049.

79. Crich, D. and Patel, M. Carb. Res. 2006, 341, 1467-1475.

80. Kim, Y.; Kim, Y. H.; Kang, S. B. and Ahn, E. J. Tetrahedron Lett. 1996, 37, 9317-9320. 
81. Casarini, A.; Dembech, P.; Lazzari, D.; Marini, E.; Reginato, G.; Ricci, A. and Seconi, G. J. Org. Chem. 1993, 58, 5620-5623.

82. Nelson, J. H. and Sveshnikov, N. N. Magn. Reson. Chem. 1997, 35, 209-212.

83. Rydzewski, R. M. and Maag, H. J. Org. Chem. 1992, 57, 5823-5831.

84. Silverstein, R. M. and Webster, F. X. Spectrometric Identification of Organic Compounds. Sixth Edition, John Wiley and Sons, New. York-London-Sydney. 1998.

85. Fraser-Reid, B. and Tulshian, D. B. J. Org. Chem. 1984, 49, 518-522.

86. Fairbanks, A.; Goddage, Y.; Chambers, D. and Evans, G. Org. Biomol. Chem. 2003, 1, 3772-3786.

87. Fairbanks, A.; Chambers, D. and Evans, G. Tetrahedron: Asymmetry 2005, 16, $45-55$.

88. Damha, M. J. and Sabitino, D. J. Am. Chem. Soc. 2007, 129, 8259-8270.

89. Yadav, J. S.; Reddy, B. V. S.; Rao, C. V.; Chand, P. K. and Prasad, A. R. Synlett 2001, 1638-1640.

90. Hayashi, M.; Kawabata, H. and Kubo, S. Carbohydr. Res. 2001, 333, 153-158.

91. Danishefsky, S.; Halcomb, R.; Wittman, M.; Olson, S.; Golik, J.; Wong, H. and Vyas, D. J. Am. Chem. Soc. 1991, 113, 5080-5082.

92. Danishefsky, S.; Wittman, M. and Halcomb, R. J. Org. Chem. 1990, 55, 19791981.

93. Isobe, M. and Huang, G. Tetrahedron 2001, 57, 10241-10246.

94. Cusk, R.; Schaade, M. and Krieger, C. Tetrahedron 1996, 52, 6397-6408.

95. Keijbets, M. and Pilnik, W. Carbohydr. Res. 1974, 33, 359-362.

96. Bemiller, J. N. and Kumari, G. V. Carbohydr. Res. 1972, 25, 419-428.

97. Doyle, M. P.; McOsker, C. C. and West, C. T. J. Org. Chem. 1976, 41, 13931396. 
98. Peczuh, M. W.; DeMatteo, M. P.; Snyder, N. L.; Morton, M.; Baldisseri, D. M. and Hadad, C. H. J. Org. Chem. 2005, 70, 24-38.

99. Levy, D. E. and Fugedi, P. The Organic Chemistry of Sugars 2006.

100. Northcote, P. T. and West, L. M. J. Org. Chem. 2000, 65, 445-449.

101. Hood, K. A.; L.M., W.; Rouwe, B.; Northcote, P. T.; Berridge, M. V.;

Wakefield, S. J. and Miller, J. H. Cancer Research 2002, 62, 3356-3360.

102. De Brabander, J. K.; Laio, X. and Wu, Y. Angew. Chem. Int. Ed. 2003, 42, $1686-1690$.

103. Taylor, R. E. and Jin, M. Org. Lett. 2005, 7, 1303-1305.

104. Hanzlik, R. P. Organic Syntheses 1977, 56, 112.

105. Sharpless, K. B. and Katsuki, T. J. Am. Chem. Soc. 1980, 102, 5974-5976.

106. Swern, D. Chem. Rev. 1949, 45, 16-25.

107. Schroder, M. Chem. Rev. 1980, 80, 187-213.

108. Sharpless, K. B.; Wang, Z.-M. and X-L, Z. Tetrahedron Lett. 1993, 34, 22672270.

109. Brown, H. C. and Subba Rao, B. C. J. Am. Chem. Soc. 1956, 78, 5694-5695.

110. Brown, H. C. Organic Syntheses via Boranes 1975, Wiley, New York.

111. Miller, A. E. G.; Biss, J. W. and Schwartzman, L. H. J. Org. Chem. 1959, 24, $627-630$

112. Naf, R. and Velluz, A. J. Ess. Oil. Res. 1991, 3, 165-172.

113. Escher, S. and Niclass, Y. Helv. Chim. Acta. 1991, 74, 179-188.

114. Shank, R. S. and Shechter, H. J. Org. Chem. 1959, 24, 1825-1826.

115. Hennion, G. F. and Sheehan, J. J. J. Am. Chem. Soc. 1949, 71, 1964-1966.

116. Swern, D.; Mancuso, A. J. and Huang, S.-L. J. Org. Chem. 1979, 43, 24802482. 
117. Pilgrim, W. Towards the synthesis of Quince Oxepane 2003, Victoria University of Wellington, School of Chemical and Physical Sciences Honours report.

118. Luh, T.-Y.; Cheng, W.-L.; Shaw, Y.-J.; Yeh, S.-M.; Kanakamma, P.; Chen, Y.H.; Chen, C.; Shieu, J.-C.; Yiin, S.-J.; Lee, C.-H. and Wang, Y. J. Org. Chem. 1999, 64, 532-539.

119. Corey, E. J. and Suggs, W. Tetrahedron Lett. 1975, 16, 2647-2650.

120. Dess, D. B. and Martin, J. C. J. Org. Chem. 1983, 48, 4155-4156.

121. Barton, D. H. R. and McCombie, S. W. J. Chem. Soc., Perkin Trans. 1 1975, 16, 1574-1585.

122. Vatele, J.-M. Tetrahedron Lett. 2006, 47, 715-718.

123. Buchanan, J. G.; Edgar, A. R.; Rawson, D. I.; Shahidi, P. and Wightman, R. H. Carb. Res. 1982, 100, 75-86.

124. Pausacker, K. J. Chem. Soc. 1953, 107-109.

125. Gurjar, M.; Kumar, P. and Venkateswara, R. Carb. lett. 2001, 4, 103-109. 